ISBN / EAN: 978-90-365-2895-5

DOI: $10.3990 / 1.9789036528955$

\title{
HYDROGEN STORAGE MATERIALS
}

\section{A FIRST-PRINCIPLES STUDY}

\author{
SÜLEYMAN ER
}




\section{HYDROGEN STORAGE MATERIALS: \\ A FIRST-PRINCIPLES STUDY}

Süleyman Er 


\section{Composition of the Graduation Committee}

\author{
Prof. Dr. V. Subramaniam \\ Prof. Dr. P. J. Kelly \\ Dr. G. H. L. A. Brocks \\ Prof. Dr. R. Griessen \\ Prof. Dr. Ir. J. W. M. Hilgenkamp \\ Prof. Dr. H. Jónsson \\ Prof. Dr. Ir. L. Lefferts \\ Dr. Ir. G. A. de Wijs
}

\author{
University of Twente, Chairman \\ University of Twente, Promoter \\ University of Twente, Assistant Promoter \\ VU Amsterdam \\ University of Twente \\ University of Iceland \\ Brown University \\ University of Twente \\ Radboud University Nijmegen
}

This work is part of the research programs of Advanced Chemical Technologies for Sustainability (ACTS), and the Stichting voor Fundamenteel Onderzoek der Materie (FOM), which are financially supported by Nederlandse Organisatie voor Wetenschappelijk Onderzoek (NWO).

The work described in this thesis is carried out at the Computational Materials Science (CMS) Group, Faculty of Science and Technology (TNW) and the MESA+ Institute for Nanotechnology of the University of Twente (UT).

Hydrogen storage materials: A first-principles study.

S. Er,

Ph. D. Thesis University of Twente, Enschede.

ISBN/EAN: 978-90-365-2895-5

DOI: $10.3990 / 1.9789036528955$

(C) S. Er, 2009

Printed by: Gildeprint Drukkerijen BV, Enschede, The Netherlands. 


\title{
HYDROGEN STORAGE MATERIALS: \\ A FIRST-PRINCIPLES STUDY
}

\author{
DISSERTATION
}

to obtain

the degree of doctor at the University of Twente, on the authority of the rector magnificus,

Prof. Dr. H. Brinksma,

on account of the decision of the graduation committee, to be publicly defended

on Thursday $29^{\text {th }}$ of October 2009 at 15:00

$$
\text { by }
$$

\section{Süleyman Er}

born on May $31^{\text {st }}, 1980$

in Civril, Turkey 
This doctoral dissertation is approved by:

Prof. Dr. P. J. Kelly Promoter

Dr. G. H. L. A. Brocks Assistant Promoter 
VERITAS 



\section{Contents}

1 Introduction $\quad 1$

1.1 Pressurized gas . . . . . . . . . . . . . . . . 3

1.2 Cryogenic liquid . . . . . . . . . . . . . . . . 3

1.3 Metal hydrides . . . . . . . . . . . . . . . . . . 3

1.4 Complex metal hydrides . . . . . . . . . . . . . . . 4

1.5 Other hydrogen storage materials . . . . . . . . . . . 5

Bibliography ..................... 8

2 Methodology $\quad 11$

2.1 The Schrödinger Equation . . . . . . . . . . . . . . . . . 11

2.2 Born-Oppenheimer Approximation . . . . . . . . . . . . . . 12

2.3 Density Functional Theory: DFT . . . . . . . . . . . . . . . . 13

2.4 Exchange and Correlation Functionals . . . . . . . . . . . . . . . 15

2.5 Pseudopotential Approach . . . . . . . . . . . . . . . 16

2.6 Periodicity vs. non-Periodicity . . . . . . . . . . . . . 17

2.7 Numerical Calculations . . . . . . . . . . . . . . . . . . . . 20 2

Bibliography ....................... 24

Part - A Atomic Hydrogen Storage 27

3 Hydrogen storage in magnesium-transition metal compounds 29

3.1 Abstract ....................... 29

3.2 Introduction . . . . . . . . . . . . . . . . . . . . 29

3.3 Computational Methods and Test Calculations . . . . . . . . . . . . 30

3.4 Results $\mathrm{Mg}_{x} \mathrm{TM}_{(1-x)} \mathrm{H}_{2} \ldots \ldots \ldots \ldots \ldots . \ldots \ldots$

3.5 Discussion . . . . . . . . . . . . . . . . . . . . 40

3.6 Summary . . . . . . . . . . . . . . . . . . . . . . . . . . . . . . . . . . . .

Bibliography ........................ 45 
4 First principles modelling of magnesium titanium hydrides 49

4.1 Abstract . . . . . . . . . . . . . . . . . . . 49

4.2 Introduction . . . . . . . . . . . . . . . . . . . . . . . . . . . . . . . . . . .

4.3 Computational Details . . . . . . . . . . . . . . . 51

4.4 Simple Dihydrides . . . . . . . . . . . . . . . . . . . 54

$4.5 \mathrm{Mg}_{x} \mathrm{Ti}_{(1-x)} \mathrm{H}_{2} \ldots \ldots \ldots \ldots \ldots \ldots \ldots$

4.6 Summary and Conclusions . . . . . . . . . . . . . 71

Bibliography ........................ 72

5 A computational study of hydrogen storage in Mg-Ti-X (X =Al, Si) 77

5.1 Abstract . . . . . . . . . . . . . . . 77

5.2 Introduction . . . . . . . . . . . . . . . . . . . . . . . . . . . .

5.3 Computational methods . . . . . . . . . . . . . . . . 79

$5.4 \mathrm{Mg}$-Ti-Al,Si metals . . . . . . . . . . . . . . . . . . 79

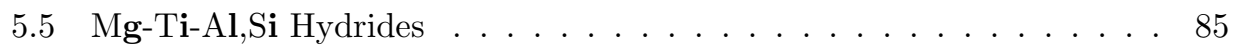

Bibliography ......................... 89

$\begin{array}{lll}\text { Part - B } & \text { Molecular Hydrogen Storage } & 93\end{array}$

6 Hydrogen storage by polylithiated molecules and nanostructures $\quad 95$

6.1 Abstract . . . . . . . . . . . . . . . . . . . 95

6.2 Introduction . . . . . . . . . . . . . . . . . . . . . . 95

6.3 Computational Details . . . . . . . . . . . . . . . . . 97

6.4 Results and Discussion . . . . . . . . . . . . . . . . . . . . 98

6.5 Conclusions . . . . . . . . . . . . . . . . . 107

Bibliography ......................... 107

7 DFT study of planar boron sheets: A new template for hydrogen storage $\mathbf{1 1 1}$

7.1 Abstract . . . . . . . . . . . . . . . . . 111

7.2 Introduction . . . . . . . . . . . . . . . . . . . 111

7.3 Computational Details . . . . . . . . . . . . . . . . . . . 114

7.4 Results and Discussion . . . . . . . . . . . . . . . . . 115

7.5 Conclusions . . . . . . . . . . . . . . . . . . 125

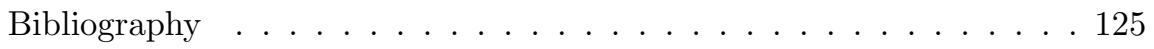

8 Lightweight metal doping of metal-organic frameworks: Implications for hydrogen storage

8.1 Introduction . . . . . . . . . . . . . . . . . . . . . . . 129

8.2 Computational Methods . . . . . . . . . . . . . . . . . 130

8.3 Results . . . . . . . . . . . . . . . . . . . . 130

Bibliography ......................... 134 
9 Summary

List of publications

Short curriculum vitæ

143 



\section{Chapter 1}

\section{Introduction}

"I will get right to the point. Energy is the single most important problem facing humanity today. We must find an alternative to oil. We need to somehow provide clean, abundant, low-cost energy throughout the world to the 6 billion people that live on the planet today, and the $10+$ billion that are expected by the middle of this century. The cheaper, cleaner, and more universally available this new energy technology is, the better we will be able to avoid human suffering, and the major upheavals of war and terrorism."

- Richard E. Smalley

As quoted above, the world needs a new source of energy that is cheap, clean and renewable. Hydrogen can be the next generation of fuels since it has a heating value $(39.3 \mathrm{kWh} / \mathrm{kg})$, which is already much higher than the most of the common fuels that are already in use today, such as gasoline $(13.3 \mathrm{kWh} / \mathrm{kg})$, propane $(14.0 \mathrm{kWh} / \mathrm{kg})$ or methane $(15.3 \mathrm{kWh} / \mathrm{kg})$. Hydrogen is abundant in the universe and on our mother earth, but, more importantly, it is regenerative and thus sustainable. If hydrogen is burned (with oxygen) to produce energy in a fuel cell or a combustion engine the only product is pure water vapor, $\mathrm{H}_{2} \mathrm{O}_{(\text {gas })}$. A hydrogen cycle based on renewable sources is entirely pollution free, i.e., it has zero $\mathrm{CO}_{2}$ emissions to the atmosphere. If produced from fossil fuels, on site $\mathrm{CO}_{2}$ capture is also feasible. Combination of all these properties opens a way of supplying more energy to the whole world and reduce the impact on the environment.

Today, more than $85 \%$ of the world energy demand is covered by fossil based fuels. These include coal, natural gas, and petroleum and its derivatives. Fig. 1.1 shows the decomposition of world energy consumption by fuel type. The world's energy demand is predicted to increase by $70 \%$ from the year 2000 to 2030. Fossil based fuels are expected to supply a significant portion of the demand also in the coming years. However, the amount fossil fuels is limited, and cannot be the ultimate solution to the 


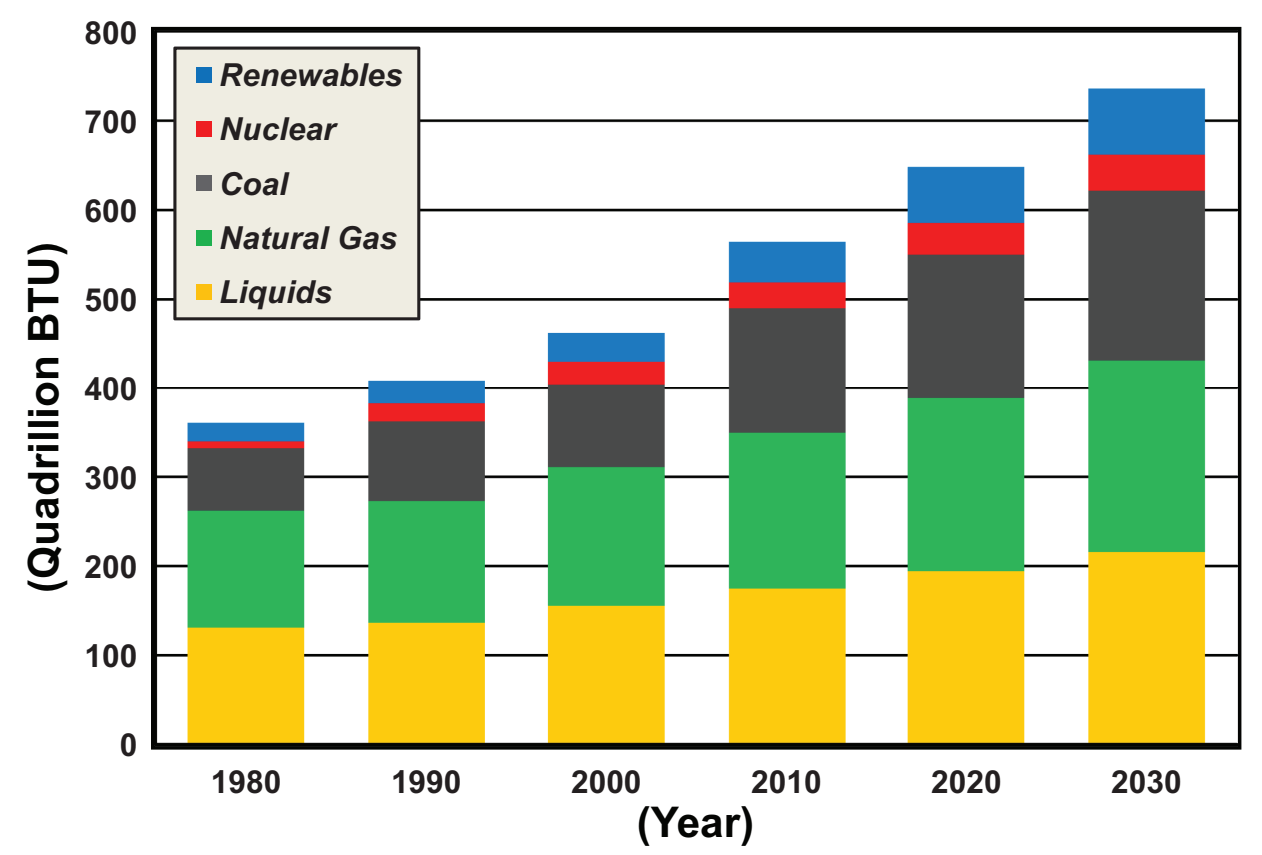

Figure 1.1: World energy consumption by fuel type. Renewables are the fastest growing source of world energy with a projected annual rate of $3.0 \%$, whereas the liquids are the worlds slowest growing source of energy with an annual rate of $0.9 \%$. [Source: US Energy Information Administration, Report No.:DOE/EIA-0484 (May 2rth, 2009)]

energy problem in the long-term. Additionally, the liquid fuel market is vulnerable to fluctuations in prices and is therefore too risky to rely on. The environmental consequences of burning fossil fuels at a larger scale are also not hard to imagine. It is predicted that when all the fossil fuel reserves are consumed, the $\mathrm{CO}_{2}$ released will raise the atmospheric temperature by $6^{\circ} \mathrm{C}$ [1]. Such a rise in temperature can harmfully affect the natural life on mother earth. According to Fig. 1.1 the fastest growing source of world energy are the renewables. It is expected to stay like this for the coming years as well. Unfortunately, the share of the renewable energy sources is still small, and definitely not enough to satisfy the world's energy demand entirely. The problems associated with renewable energy systems can be classified into three main categories: $(i)$ the efficient conversion of energy into electricity, $(i i)$ the storage of energy in the form of hydrogen, and (iii) the conversion of hydrogen into work.

In this study we focus on the storage aspects of hydrogen. Under normal conditions hydrogen is a nontoxic, low density gas and composed of diatomic molecules $\left(\mathrm{H}_{2}\right)$. The atomic form of hydrogen is very reactive and forms numerous compounds with many other elements, such as oxygen (water). Since a hydrogen atom consists of 
a single proton and a single electron, hydrogen is the lightest of all the elements. It can diffuse easily in air or through other substances. Efficient hydrogen storage requires innovative methods, and the development of these methods requires research at a fundamental level. The following sections introduce the most common hydrogen storage materials that are at the focus of present scientific interests.

\subsection{Pressurized gas}

High pressure gas tanks are the most common storage systems used in today's world. The commercial tanks made from steel are operable up to 200 bars [2], whereas, composite cylinders developed so far can stand pressures up to 800 bars and contain $36 \mathrm{~kg}$ hydrogen per $\mathrm{m}^{3}$ volume. The gravimetric hydrogen densities are, however, markedly reduced due to the necessity of using thick walled cylinders that can withstand such high pressures. Moreover, the energetic cost of pressurizing hydrogen is substantial. At room temperature it requires about $2.21 \mathrm{kWh} / \mathrm{kg}$ to reach 800 bars. Furthermore, the safety of high pressure gas tanks is an issue of concern especially if they are used on vehicles or at stations located nearby a densely populated area.

\subsection{Cryogenic liquid}

Space technology has made use of hydrogen as a fuel in liquid form for several years [3. Similar to any other gas, storing hydrogen as liquid takes less space than storage as pressurized gas. The volumetric density of liquid hydrogen is $70 \mathrm{~kg}$ per $\mathrm{m}^{3}$, which is almost twice of that of the pressurized gas at 800 bars. Once liquefied it can be maintained as a liquid in thermally insulated containers. However, liquid hydrogen has an extremely low boiling temperature of $20 \mathrm{~K}$, which is actually the second lowest boiling temperature in nature after Helium [4. Consequently, it requires very sophisticated insulation techniques to keep hydrogen in liquid form. Taking into account of the high energetic cost of hydrogen liquefaction, about 15.2 $\mathrm{kWh} / \mathrm{kg}$, together with the difficulties in maintenance, one can conclude that the liquid hydrogen option has no real practical use.

\subsection{Metal hydrides}

Most of the metals in the periodic table, their alloys or intermetallic compounds react with hydrogen to form metal hydrides. In this sense, the host metallic systems act like hydrogen sponges. The bonding between hydrogens and the metals can range from very covalent to very ionic as well as multi-centered bonds and metallic bonding. The classification of metal hydrides in terms of bonding, however, is not usually sharp, since in some cases several kinds of bonding can be observed in a single metal hydride system. 
At certain temperature and pressure conditions large quantities of hydrogen can be absorbed by such metallic systems. When needed as fuel in a fuel cell or combustion engine to generate energy, hydrogen can be released from metal hydrides simply by increasing the temperature. Some metal hydrides can store hydrogen in a density higher than that of liquid hydrogen. The possibility of storing hydrogen in a more compact and safer way compared to pressurized gas and cryogenic liquid is exciting. The (de)hydrogenation reaction can be represented in a simple way:

$$
M+\frac{y}{2} \mathrm{H}_{2} \rightleftharpoons M \mathrm{H}_{\mathrm{y}}
$$

where $M$ is the host storage metal or metal mixture. Here, the thermodynamics play a crucial role in determining whether the material is suitable for storing hydrogen or not. At equilibrium, the change in Gibbs Free Energy ( $\Delta G=\Delta H-T \Delta S$ ) between the metal hydride and the hydrogen gas is zero. The entropy of the metal hydride can be neglected compared to that of the hydrogen gas. The entropy of hydrogen gas at standard conditions $\left(25^{\circ} \mathrm{C}\right.$ and 1 bar pressure) is $130.7 \mathrm{~J} / \mathrm{mol} \cdot \mathrm{K}[5]$. For equilibrium under standard conditions, this leads to a formation enthalpy, $\Delta H$, of the hydride of $-39 \mathrm{~kJ} / \mathrm{mol}$ (or $-0.40 \mathrm{eV}$ ).

Besides suitable thermodynamic properties and volumetric densities, another important issue regarding metal systems is the total weight. In fact, only lightweight metals or their compounds are promising for onboard hydrogen storage. Using a a significant amount of heavy metal in intermetallic compounds has no use since it will result a reduction in hydrogen gravimetric density. The time to load the storage material with hydrogen at a fueling station and unload it onboard is also a concern. Since the formation of metal hydrides and their decomposition back into molecular hydrogen is a chemical process involving lots of bond breaking and formation, storage materials that can demonstrate fast hydrogen kinetics are needed. Recent research on metal hydrides focus on modifying the metal hydride compositions in such a way that the thermodynamics is favorable, hydrogen kinetics is fast, while a meaningful hydrogen gravimetric density is preserved.

\subsection{Complex metal hydrides}

Hydrides formed by a combination of metals or metalloids are called complex metal hydrides. In such materials hydrogen atoms are bonded covalently to a metal or metalloid atom to form an anion. This anion is then bonded ionically to a metal cation present, to form a complex metal hydride [6].

In general, complex metal hydrides have the formula $\mathrm{A}_{\mathrm{x}} \mathrm{M}_{\mathrm{y}} \mathrm{H}_{\mathrm{z}}$, where " $\mathrm{A}$ " is an alkali metal or alkaline earth metal cation or cation complex, and "M" is a metal or metalloid. Well known examples feature anions of hydrogenated group 3 elements, in particular boron and aluminium. Compounds such as $\mathrm{LiBH}_{4}$ (lithium borohydride), and $\mathrm{NaAlH}_{4}$ (sodium alanate) are among the most widely studied. The variety in complex metal hydrides is very large. The possibility of forming complex metal hydrides using lightweight elements opens a promising route to achieve a very high 
hydrogen content by weight, e.g., $\mathrm{LiBH}_{4}$ contains 18 wt \% hydrogen. Accordingly, there is an increasing interest to explore complex metal hydride systems and their subsequent optimization for practical use. Combining several complex hydrides into one storage system might improve the storage characteristics, but the complexity of reaction mechanisms requires further fundamental research on such materials.

\subsection{Other hydrogen storage materials}

There are several other materials that are potentially interesting for hydrogen storage. In this section a simple description is given of such materials.

\subsubsection{Clathrates}

A clathrate is a molecule that traps a second kind of molecule inside. In this case, hydrogen is the guest molecule stored inside clathrates. Large clathrate cages can accommodate multiple hydrogen molecules. Specific examples of the hydrogen storage clathrate family are the clathrate hydrates. They are formed by exerting huge pressures, approximately 2000 bars, to water. The water molecules then form a crystal with cages that can be filled with hydrogen molecules. The desorption mechanism of hydrogen from clathrates involves a structural break down of the clathrate crystal. Recent research focuses on clathrates that can be operated at lower pressures.

\subsubsection{Zeolites}

Zeolites are microporous inorganic compounds. Some zeolites occur naturally, but most of them are synthesized on an industrial scale. The application areas of zeolites are very diverge, including medical, agricultural, nuclear and petrochemical industries. The beauty of zeolites comes from their ability to selectively sort molecules based primarily on a size exclusion process. The pore structure of zeolites is regular with effective pore sizes between 3 and $10 \AA$ []. Hydrogen storage inside zeolite pores faces technical difficulties arising from thermodynamics and kinetics. Moreover, zeolites are usually formed with heavy elements, and therefore hydrogen gravimetric densities are limited.

\subsubsection{Nanoporous materials}

Recent advances in nanotechnology inspire researchers in developing new hydrogen storage systems. Reasonably inexpensive nano-materials with large internal surfaces can be built from lightweight elements, such as boron, carbon and nitrogen. The storage takes place as hydrogen molecules are adsorbed on the surface of the solids [8]. The possibility of storing hydrogen in molecular form is advantageous over chemical storage in atomic form, which requires dissociation of the hydrogen bond and the formation of a hydride. In this section a brief introduction is given on nanomaterials that are promising for molecular hydrogen storage. 


\subsection{3.a Planar systems}

Some materials have a layered structure, which is the main driving force for studies on single layers for hydrogen storage. As an example of a planar system one can consider a single sheet of graphite, namely graphene [9, 10. Theoretical calculations show that graphene layers spaced between 6 and 7 Angstroms apart store hydrogen at room temperature and 100 bars [11. However, this is only possible if the interplanar distances can be fixed at an appropriate value that maximizes the stored hydrogen content. Spacer molecules can be used for this purpose, although an ideal one has not been reported yet. In order for graphene to become a strong contender for practical hydrogen storage, thermodynamical issues need to be resolved as well. The interaction of hydrogen molecules with the layers needs to be increased in order to enable operating under moderate temperature and pressure conditions. In addition, extending the storage densities to well over a monolayer hydrogen coverage would be interesting. Recently, there are numerous studies in this field [12 18].

\subsection{3.b Curved systems}

Carbon structures with curvature, such as single-walled carbon nanotubes (SWNT) [19-22] and buckyballs $23-26$ ] are among the most widely investigated nano-materials for hydrogen storage. The presence of a high specific surface area is promising for physical and chemical optimization of such materials. Carbon-only structures, and their derivatives obtained through doping, are the main stream materials considered so far. Compared to the planar systems discussed in section 1.5.3.a, structures with curvatures faces the same challenges in the optimization process to store hydrogen. These can be categorized into two main categories. Firstly, the binding energies of hydrogen molecules to naked curved systems (carbon only, or doped systems with similar curvatures, such as boron or nitrogen doped SWNTs or buckballs) is in the physisorption range. Therefore these unpractically low binding energies have to be increased. Secondly, similar to the planar systems the hydrogen coverage densities need to be enhanced to well over monolayer values.

The usual approach to the above mentioned problems is doping nano-materials with metals. Considering the gravimetric hydrogen density issue, it is only the lightweight metals that are potentially interesting for doping. The challenge is to find metals that can bind to SWNTs or buckyballs strong enough so that they are immobilized on the surface, and resist to metal clustering. The density of the metals covering the surface is also important and needs to be adjusted to an optimum value. That is, it should be high enough to capture as much hydrogen as possible, but low enough to preserve the hydrogen gravimetric density by not influencing the total system weight to a great extend. The interaction between the hydrogen molecules and metal atoms deposited on a surface can be electrostatic, chemisorption or of Kubas type $[27-30]$. 


\subsection{3.c Metal-Organic Frameworks: MOFs}

Metal-Organic Frameworks (MOFs) are crystalline compounds consisting of metal ions or metal oxide clusters, coordinated with rigid organic molecules, forming regular three dimensional (3-D) structures [31. MOFs are stable and highly porous systems. Additionally, they are among the lowest density crystalline materials, thanks to the presence of pores inside the lattice and the usage of mainly lightweight elements as building blocks. All these properties together make MOFs ideal candidates to store gases, like as hydrogen, methane or carbon dioxide [32 45. Although the properties of gas kinetics depends on the size of the pores, in general MOFs are considered as systems allowing fast diffusion. This is due to the fact that the pores in 3-D form a continuous network.

The interaction of hydrogen molecules with the material shows different properties depending on the metal and organic components used in the construction of network. MOFs without defects in their crystal structures can bind several hydrogen molecules, but the binding interactions are usually weak, similar to other physisorption nanomaterials. Increasing the $\mathrm{H}_{2}$ binding energies is a current focus of research on MOF systems. Some of these methods can briefly explained as follows:

- $(i)$ It is believed that MOFs with very large pores are not practical for massive hydrogen store. The reasoning is that the $\mathrm{H}_{2}$ molecules close to the center of the pores are weakly interacting with the framework, consequently the binding energies are very low. The reduction of the pore size can be accomplished by penetrating MOFs. It is, however, is not so clear yet whether an increase in hydrogen binding energies as a consequence of penetration will offset the loss of free volume and its effects on the total hydrogen uptake.

- (ii) The second method involves catalyst initiated dissociation of $\mathrm{H}_{2}$ and subsequent migration of atomic hydrogen onto the material surface. This process is called hydrogen spillover. However, the process of spillover in MOFs is largely unknown, and remains to be explored at a fundamental level. The recyclability and kinetics remain the main concerns for hydrogen stored via spillover in MOFs.

- (iii) Exposing metal atoms is another approach to improve interactions between hydrogen molecules and the MOFs. This is accomplished either by creating vacancies within the framework itself, or pumping extra metal atoms into the MOF lattice. Accordingly, hydrogen molecules are expected to be captured via non-van der Waals binding, such as Kubas type or electrostatic interactions. In principle, the strength of interactions are expected to increase to a range between physisorption and chemisorption. Unfortunately, the number of hydrogen molecules that can bind to a defect site is limited, and the only way to increase hydrogen densities is to create as many defect sites as possible. Defects, however, may lead to deformations in the framework structure and can harmfully affect the gas diffusion. Recent theoretical studies claim that MOFs with penetrated lightweight metal ions, such as Li, show improved hydrogen binding energies [46, 47. 
To sum up, the ability to modify the metallic and organic components of metalorganic frameworks in a way to optimize the hydrogen storage properties is promising. Lately, numerous experimental and theoretical studies focus on gas storage in MOFs.

\section{Bibliography}

[1] S. Arrhenius, Philos. Mag. 41, 237 (1896).

[2] L. Schlapbach and A. Züttel, Nature 414, 353 (2001).

[3] S. Sherif, N. Zeytinoglu, and T. Veziroglu, Int. J. Hydrogen Energy 22, 683 (1997).

[4] A. M. James and M. P. Lord, Macmillan's chemical and physical data (London: Macmillan, 1992).

[5] M. W. Chase, J. Phys. Chem. Ref. Data 9, 1.

[6] A. F. Holleman and E. Wiberg, Holleman-Wiberg Inorganic Chemistry (San Diego: Academic Press, 2001).

[7] C. R. A. Catlow, Modelling of structure and reactivity in zeolites (London: Academic Press, 1992).

[8] A. Züttel, Mater. Today 6, 24 (2003).

[9] A. Geim and K. Novoselov, Nat. Mater. 6, 183 (2007).

[10] A. Geim and A. MacDonald, Phys. Today 60, 35 (2007).

[11] S. Patchkovskii et al., Proc. Natl. Acad. Sci. U.S.A. 102, 10439 (2005).

[12] J. S. Arellano, L. M. Molina, A. Rubio, and J. A. Alonso, J. Chem. Phys. 112, 8114 (2000).

[13] T. Heine, L. Zhechkov, and G. Seifert, Phys. Chem. Chem. Phys. 6, 980 (2004).

[14] W. Q. Deng, X. Xu, and W. A. Goddard, Phys. Rev. Lett. 92, 166103 (2004).

[15] D. Henwood and J. D. Carey, Phys. Rev. B 75, 245413 (2007).

[16] N. Park, S. Hong, G. Kim, and S. H. Jhi, J. Am. Chem. Soc. 129, 8999 (2007).

[17] G. Kim, S. H. Jhi, and N. Park, Appl. Phys. Lett. 92, 013106 (2008).

[18] C. Ataca, E. Aktürk, S. Ciraci, and H. Ustunel, Appl. Phys. Lett. 93, 043123 (2008).

[19] P. Chen, X. Wu, J. Lin, and K. L. Tan, Science 285, 91 (1999).

[20] C. Liu et al., Science 286, 1127 (1999). 
[21] R. Yang, Carbon 38, 623 (2000).

[22] E. Lee, Y. Kim, Y. Jin, and K. Chang, Phys. Rev. B 66, 73415 (2002).

[23] Y. H. Kim, Y. Zhao, A. Williamson, M. J. Heben, and S. B. Zhang, Phys. Rev. Lett. 96, 16102 (2006).

[24] Q. Sun, P. Jena, Q. Wang, and M. Marquez, J. Am. Chem. Soc. 128, 9741 (2006).

[25] K. R. S. Chandrakumar and S. K. Ghosh, Nano Lett. 8, 13 (2008).

[26] Q. Sun, Q. Wang, and P. Jena, Appl. Phys. Lett. 94, 013111 (2009).

[27] J. Niu, B. K. Rao, and P. Jena, Phys. Rev. Lett. 68, 2277 (1992).

[28] L. Gagliardi and P. Pyykkö, J. Am. Chem. Soc. 126, 15014 (2004).

[29] E. Durgun, S. Ciraci, W. Zhou, and T. Yildirim, Phys. Rev. Lett. 97, 226102 (2006).

[30] R. C. Lochan and M. Head-Gordon, Phys. Chem. Chem. Phys. 8, 1357 (2006).

[31] O. Yaghi et al., Nature 423, 705 (2003).

[32] N. L. Rosi et al., Science 300, 1127 (2003).

[33] X. Zhao et al., Science 306, 1012 (2004).

[34] J. Rowsell, J. Eckert, and O. Yaghi, J. Am. Chem. Soc. 127, 14904 (2005).

[35] J. Rowsell, A. Millward, K. Park, and O. Yaghi, J. Am. Chem. Soc. 126, 5666 (2004).

[36] J. Rowsell, E. Spencer, J. Eckert, J. Howard, and O. Yaghi, Science 309, 1350 (2005).

[37] J. Rowsell and O. Yaghi, J. Am. Chem. Soc. 128, 1304 (2006).

[38] J. Rowsell and O. Yaghi, Angew. Chem., Int. Ed. 44, 4670 (2005).

[39] A. Wong-Foy, A. Matzger, and O. Yaghi, J. Am. Chem. Soc 128, 3494 (2006).

[40] S. S. Kaye, A. Dailly, O. M. Yaghi, and J. R. Long, J. Am. Chem. Soc 129, 14176 (2007).

[41] J. G. Vitillo et al., J. Am. Chem. Soc. 130, 8386 (2008).

[42] W. Zhou, H. Wu, and T. Yildirim, J. Am. Chem. Soc. 130, 15268 (2008).

[43] M. Dinca and J. R. Long, Angew. Chem., Int. Ed. 47, 6766 (2008).

[44] A. Dailly, J. Vajo, and C. Ahn, J. Phys. Chem. B 110, 1099 (2006). 
[45] J. Liu et al., J. Phys. Chem. C 100, 2911 (2008).

[46] S. Han and W. Goddard III, J. Am. Chem. Soc. 129, 8422 (2007).

[47] A. Blomqvist, C. M. Araujo, P. Srepusharawoot, and R. Ahuja, Proc. Natl. Acad. Sci. U.S.A. 104, 20173 (2007). 


\section{Chapter 2}

\section{Methodology}

An appropriate description of the physical and chemical properties of matter is a complicated task. The problem involves interacting nuclei and electrons, and in some cases an external field. Quantum mechanics is a set of principles that predicts how the particles behave, given the elementary properties such as the rest masses and charges. It is the most successful and powerful theory ever created. In the heart of this monumental scientific achievement lies the Schrödinger Equation. In principle, by solving the Schrödinger equation one can access all the properties of a material of any size. However, the problem becomes practically unsolvable as the number of interacting particles increases, which is the case for most real-life situations.

The methodology chapter starts with a short introduction of the Schrödinger Equation, which is followed by the Born-Oppenheimer Approximation. Density Functional Theory, which is one of the leading electronic structure methods developed so far to investigate many-body systems (i.e., atoms, molecules, clusters or condensed phases), is discussed next. Exchange and correlation functionals are discussed in Section 2.4. Section 2.5] summarizes the usefulness of pseudopotential approach. Technical details on modeling infinite periodic systems, as well as the basis set used in the present calculations, are mentioned in Section 2.6. The last section of the chapter (Section 2.7) includes the details of calculations of some physical properties.

\subsection{The Schrödinger Equation}

At the beginning of the twentieth century, wave-like behavior of atomic particles observed in experiments inspired theoreticians in using wave equations to describe the behavior of physical systems. In the year 1926 Erwin Schrödinger formulated a fundamental equation to describe the quantum aspects of matter. The many body form of the time independent Schrödinger equation consisting of $M$ nuclei and $N$ electrons is written as: 


$$
\hat{H} \psi_{i}\left(\mathbf{r}_{1}, \mathbf{r}_{2}, \ldots, \mathbf{r}_{N}, \mathbf{R}_{1}, \mathbf{R}_{2}, \ldots, \mathbf{R}_{M}\right)=E_{i} \psi_{i}\left(\mathbf{r}_{1}, \mathbf{r}_{2}, \ldots, \mathbf{r}_{N}, \mathbf{R}_{1}, \mathbf{R}_{2}, \ldots, \mathbf{R}_{M}\right),
$$

where, $\hat{H}$ denotes the Hamiltonian, and $\psi_{i}$ the wave function in terms of all the electronic (r) and nuclear coordinates $(\mathbf{R})$.

In principle, the above equation is applicable to any physical system regardless of size (i.e., from atoms to a whole crystal). In practice, however, a complete analytical solution is almost impossible for most real life systems, since the number of variables to deal with is determined by the $3(M+N)$ degrees of freedom. If we consider the methane molecule (chemical formula: $\mathrm{CH}_{4}$ ), then there are in total 5 atomic nuclei and 10 electrons. The time independent Schrödinger Equation for a single methane molecule consequently becomes a partial differential eigenvalue problem in 45 variables. Although the exact solution of the Schrödinger Equation is unaffordable for almost every real system, sensible approximations are used to make the most use of it.

\subsection{Born-Oppenheimer Approximation}

The Born-Oppenheimer Approximation [1] lightens the calculation of the energy and the wave function of moderate size physical systems by making use of the significant mass differences between the nuclei and the electrons. Considering the same methane molecule example mentioned in the previous section (Section 2.1), we see that mass of the nucleus is 2800 times that of all the electrons surrounding it. It is therefore possible to decouple the motion of relatively slow moving nuclei and that of the fast moving electrons. In other words, the wave function of the system can be divided into electronic and nuclear components:

$$
\psi(\mathbf{r}, \mathbf{R})=\psi_{\text {electronic }}(\mathbf{r}, \mathbf{R}) \times \psi_{\text {nuclear }}(\mathbf{R}) .
$$

In the first step of the Born-Oppenheimer Approximation the nuclei are assumed to have fixed positions, and the electronic Schrödinger Equation, which depends only on the electronic coordinates, is solved. For instance, the electronic wave function of the methane molecule depends on 30 electronic coordinates. It is assumed that for fixed nuclear positions the electrons are in their ground state. Consequently, the calculated electronic energies are functions of nuclear positions. In the second step of the Born-Oppenheimer Approximation, these electronic functions serve to calculate a potential for the nuclear component of the Schrödinger Equation. In the case of the methane example it needs to deal with only 15 variables. To sum up, the nuclei move on the potential energy surface of the electronic ground state, and the electrons follow the nuclear motion adiabatically. 


\subsection{Density Functional Theory: DFT}

Decoupling the Schrödinger Equation into electronic and nuclear components by the Born-Oppenheimer Approximation simplifies the problem. However, the electronic problem is still too complicated to be solved exactly due to the interactions between the electrons. Instead of using the 3N-dimensional Schrödinger Equation for the many electron wave function, $\psi_{i}\left(\mathbf{r}_{1}, \mathbf{r}_{2}, \ldots, \mathbf{r}_{N}\right)$, Density Functional Theory (DFT) reduces the problem into a series of coupled one-body problems. This is accomplished by using the electronic density distribution, $n(r)$, and a universal functional of the density $E_{\mathrm{xc}}[n(r)]$.

\subsubsection{Hohenberg-Kohn Theorem}

In the year 1964, P. Hohenberg and W. Kohn [2] proved that the construction of the total energy of a physical system can be expressed in terms of the electronic density. The ideas are related to earlier propositions of Thomas and Fermi in 1927, where atoms were modeled as systems with a positive potential (the nucleus) located in a uniform electron gas [3, 4]. The Hohenberg-Kohn theorem consist of two main parts:

- Theorem I: In a system of $N$ interacting particles under the influence of an external potential $V_{\text {ext }}(r)$, the ground state electron density, $n(r)$, uniquely determines the potential, $V_{\text {ext }}(r)$.

Since $n(r)$ determines $V_{\text {ext }}(r)$, then the full ground state Hamiltonian is known. Consequently, $n(r)$ completely determines all the properties of a physical system, such as the eigenfunctions, $\psi_{i}\left(\mathbf{r}_{1}, \mathbf{r}_{2}, \ldots, \mathbf{r}_{N}\right)$ and the eigenvalues, $E_{i}$. In other words, the first theorem states that the energy is a unique functional of the electron density:

$$
E[n(\mathbf{r})]=F[n(\mathbf{r})]+\int V_{\text {ext }}(\mathbf{r}) n(\mathbf{r}) d^{3} \mathbf{r} .
$$

- Theorem II: There exists a functional $F[n(r)]$ for the ground state energy, for any given $V_{\text {ext }}(r)$. The global minimum of this functional defines the exact ground state energy of the physical system, and the density that minimizes the total energy is the exact ground state density, $n_{0}(r)$.

The second theorem states that the ground state electron density, $n_{0}(r)$, minimizes the universal functional, $F$, such that the electronic energy reaches its minimum value of $E\left[n_{0}(\mathbf{r})\right]$ :

$$
E\left[n_{0}(\mathbf{r})\right]=F\left[n_{0}(\mathbf{r})\right]+\int V_{\mathrm{ext}}(\mathbf{r}) n_{0}(\mathbf{r}) d^{3} \mathbf{r} .
$$

It should be noted that $F[n(\mathbf{r})]$ depends purely on the electron density. It does not depend on the external potential, $V_{\text {ext }}(r)$. The above two theorems together form the basis of DFT. 


\subsubsection{Kohn-Sham Equations}

In order to find the ground state density, $n_{0}(\mathbf{r})$ W. Kohn and L. Sham [5] developed a set of differential equations in 1965. They start with the decomposition of the density functional given in (Eq. 2.3) into three parts:

$$
E[n(\mathbf{r})]=T_{0}[n(\mathbf{r})]+E_{H}[n(\mathbf{r})]+E_{x c}[n(\mathbf{r})]+\int V_{\text {ext }}(\mathbf{r}) n(\mathbf{r}) d^{3} \mathbf{r},
$$

where $T_{0}[n(\mathbf{r})], E_{H}[n(\mathbf{r})]$ and $E_{x c}[n(\mathbf{r})]$ define the kinetic energy, Hartree energy as a consequence of electron-electron repulsions, and exchange and correlation energies, respectively. The main idea behind the Kohn and Sham equations is to replace the kinetic energy of interacting electrons with that of an equivalent non-interacting system, and define $E_{x c}$ such, that the non-interacting system has the same ground state density as the interacting system. Within this assumption the electron density is given by the single particle wave functions, $\psi_{i, s}$ where $s$ labels the spin state,

$$
n(\mathbf{r})=\sum_{s=1}^{2} \sum_{i=1}^{N}\left|\psi_{i, s}(\mathbf{r})\right|^{2}
$$

and accordingly the kinetic energy is described as,

$$
T_{0}[n(\mathbf{r})]=\sum_{s=1}^{2} \sum_{i=1}^{N}\left\langle\psi_{i, s}(\mathbf{r})\left|-\frac{\nabla^{2}}{2}\right| \psi_{i, s}(\mathbf{r})\right\rangle .
$$

Minimizing the total energy with respect to the single particle wave functions, under the constraint that these form an orthonormal set, leads to the Kohn-Sham equations:

$$
\left[-\frac{\hbar^{2}}{2 m} \nabla^{2}+V_{\text {eff }}(\mathbf{r})\right] \psi_{i, s}(\mathbf{r})=\epsilon_{i, s} \psi_{i, s}(\mathbf{r}) .
$$

In this way, the problem of interacting particles is reduced to an equivalent problem of non-interacting particles. The effective potential used in above Eq. (2.8) has the form:

$$
V_{\text {eff }}(\mathbf{r})=e^{2} \int \frac{n(\mathbf{r})}{\left|\mathbf{r}-\mathbf{r}^{\prime}\right|} d^{3} \mathbf{r}+\frac{\delta E_{\mathrm{xc}}[n]}{\delta n(\mathbf{r})}+V_{\text {ext }}(\mathbf{r}) .
$$

In principle, the above Kohn-Sham equations are just mathematical representations used to reduce the complexity of the problem. They need to be solved using a self consistent procedure. Although the exact $E_{x c}$ functional is not known, approximate functionals have been constructed that are universal in the sense that they do not depend on the materials investigated. Without the use of special basis sets and algorithms, the computational costs of solving the Kohn-Sham equations scale as $N^{3}$, where $N$ represents the total number of Kohn-Sham orbitals. 


\subsection{Exchange and Correlation Functionals}

The Kohn-Sham approach allows the calculation of the kinetic energy term in Eq. 2.5. The only undetermined component of the equation is the exchange-correlation energy functional, $E_{x c}=E_{x}+E_{c}$. However, the exact form this functional is not known and an approximate description is used instead. Researchers still focus on generating new exchange-correlation functionals to improve the accuracy of DFT. Computational results obtained with different functionals are directly compared to experiments to decide which functionals to be used in a particular study. The details of the functionals used in this study are given below:

\subsubsection{Local Density Approximation: LDA}

In the local density approximation (LDA) general inhomogeneous electronic system is considered as locally homogeneous. For a spin-unpolarized system, local density approximation for the exchange-correlation energy is written as

$$
E_{\mathrm{xc}}^{\mathrm{LDA}}=\int n(\mathbf{r}) \varepsilon_{\mathrm{xc}}[n(\mathbf{r})] d^{3} \mathbf{r},
$$

where $n$, and $\varepsilon_{\mathrm{xc}}[(n(\mathbf{r})]$ define the electron density and exchange-correlation energy per electron, respectively. Similarly, the exchange-correlation potential is written as

$$
V_{\mathrm{xc}}(\mathbf{r})=\frac{\delta E_{\mathrm{xc}}[n(\mathbf{r})]}{\delta n(\mathbf{r})}=\varepsilon_{\mathrm{xc}}[n(\mathbf{r})]+n(\mathbf{r}) \frac{d \varepsilon_{\mathrm{xc}}[n(\mathbf{r})]}{d n(\mathbf{r})} .
$$

The most common form of LDA is based on numerical Monte Carlo calculations of the energy of homogeneous electron gases of varying densities, $\varepsilon_{\mathrm{xc}}[(n(\mathbf{r})]=$ $\varepsilon_{\mathrm{xc}}^{\text {hom }}[(n(\mathbf{r})]$ by Ceperley and Alder [6]. In this study, we make use of the Perdew and Zunger [7] parameterized form of the Ceperley and Alder exchange-correlation.

LDA calculations usually describe the chemical trends correctly. The geometrical properties such as the cell parameters, bond lengths, and bond angles are also within a few percent for systems involving covalent, ionic or metallic bonds. On the other hand, the binding energies are mostly overestimated. In LDA, the exchangecorrelation energy due to inhomogeneities in the electron density is ignored. Consequently, LDA has limitations for very inhomogeneous systems, finite systems being the most obvious ones.

\subsubsection{Generalized Gradient Approximation: GGA}

The failure of LDA in situations where the charge density undergoes rapid changes has motivated scientists to develop more advanced approximations. Deviations from the non-uniform charge density can be expressed in terms of gradients and higher spatial derivatives of the total charge density. Generalized Gradient Approximation (GGA) uses the gradient of the charge density as a correction for such deviations. The functional can be defined as a generalized form of Eq. (2.10) such that, 


$$
E_{\mathrm{xc}}^{\mathrm{GGA}}=\int n(\mathbf{r}) \varepsilon_{\mathrm{xc}}[n(\mathbf{r})] F_{\mathrm{xc}}[n(\mathbf{r}), \nabla n(\mathbf{r})] d^{3} \mathbf{r} .
$$

$F_{\mathrm{xc}}$ is dimensionless and numerous forms of it exist in literature. The most widely used forms in solid state physics are proposed by Perdew and Wang(PW91) [8] and Perdew, Burke, and Ernzerhof (PBE) [9, 10]. Unless otherwise stated, GGA calculations in this study make use of the PW91 functional. Some of the calculations are repeated with the $\mathrm{PBE}$ functional for comparison.

Compared to LDA, GGA improves the binding energies, especially when there is chemical bonding between the atoms. The description of the geometries, however, is not universally better. Similar to LDA, screening of exchange hole is not fully taken into account in GGA. Consequently, GGA cannot account for noticeable improvements to the band gap problem or to the calculation of the dielectric constants.

\subsection{Pseudopotential Approach}

The majority of the properties of a physical system are determined by its valence electrons rather than its core electrons. Therefore, in an effort to simplify the computational cost of calculations the ionic cores can be treated as frozen in their atomic configurations. In other words, the core electrons are pre-calculated in an atomic environment and kept frozen in the course of the remaining calculations. The pseudopotential (also called effective potential) approach utilizes this idea by replacing the core electrons and the strong ionic potential with a weaker pseudopotential that acts on a set of pseudo wave functions. Although the general ideas behind the pseudopotential approach are similar, there are several procedures to construct pseudopotentials, such as norm-conserving [11-13] and ultrasoft [14, 15. In this study we make use of the pseudopotentials constructed using the Projector Augmented Wave (PAW) method [16, 17] as will be discussed in the next section.

\subsubsection{Projector Augmented Waves: PAW}

In 1994, Peter Blöchl developed the so called Projector Augmented Wave (PAW) method which combines the traditions of augmented wave methods and ultra-soft pseudopotentials into a unified description [16. The most striking property of the PAW method is that the full all-electron wave function is kept and therefore the wave functions within the core regions are recovered. Additionally, advanced algorithms are used for a more efficient solution of the generalized eigenvalue problem.

In an effort to overcome the computational costs resulting from all-electron calculations, Blöchl pointed out that the true wave function, $\Psi$, and the pseudo wave function, $\tilde{\Psi}$, can be linked by a linear transformation

$$
\Psi=T \tilde{\Psi}
$$




$$
T=1+\sum_{j}\left(\left|\phi_{j}\right\rangle-\left|\tilde{\phi}_{j}\right\rangle\right)\left\langle\tilde{p}_{j}\right| .
$$

The proposed transformation operator is shown above (Eq. 2.14), where $\phi_{j}, \tilde{\phi}_{j}$, and $\tilde{p}_{j}$ are the all-electron partial waves, pseudo partial waves and PAW projector functions, respectively. Projector functions probe the character of the wave function, such as $s, p$ and $d$-type. Applying this into the all-electron wave function equation one obtains the following expression:

$$
\left|\Psi_{n}\right\rangle=\left|\tilde{\Psi}_{n}\right\rangle+\sum_{j}\left(\left|\phi_{j}\right\rangle-\left|\tilde{\phi}_{j}\right\rangle\right)\left\langle\tilde{p}_{j} \mid \tilde{\Psi}_{n}\right\rangle .
$$

As the projector functions are effectively operative only in the core regions, the all-electron wave functions are obtained from the pseudo wave functions by projecting out the core regions and replacing the pseudo partial waves by the all electron partial waves. In principle, the functions $\phi_{j}, \tilde{\phi}_{j}$, and $\tilde{p}_{j}$ can be optimized self-consistently for each system. In practice, these functions are obtained from an atomic calculation and then frozen, such that their effect can be incorporated as a fixed pseudopotential. Consequently, the computational effort is now reduced to a great extend. The PAW technique is surprisingly accurate, i.e., almost comparable to the full all-electron methods.

\subsection{Periodicity vs. non-Periodicity}

If a system obeys a set of periodic boundary conditions, this enables to model a large system using only a small part of it that is far from its edge. Crystalline bulk materials with no surfaces present are perfect examples of systems with periodicity. Such systems can be studied using unit cells to reduce the computational effort. In mathematical terms, for the potential of a periodic system operating on an electron we have:

$$
V(\mathbf{r}+\mathbf{R})=V(\mathbf{r}),
$$

for all Bravais lattice vectors $\mathbf{R}$. Since the scale of periodicity of $V$ is $\sim 10^{-9}$ meters, quantum mechanics is an essential tool to study the effect of periodicity on electronic motion.

\subsubsection{Bloch's Theorem}

In a condensed system the number of electrons can be assumed as infinite, which makes the solution of the Schrödinger equation very complicated. Felix Bloch, in year 1928, developed a theorem that enables the consideration of only the electrons within the unit cell to solve the Schrödinger equation [18, 19. The theorem states that the wave function of an electron within a perfectly periodic potential can be written as 


$$
\psi_{n \mathbf{k}}(\mathbf{r})=u_{n \mathbf{k}}(\mathbf{r}) e^{i \mathbf{k} \cdot \mathbf{r}},
$$

where $\mathbf{k}$ is the wave vector analogous to that of the wave vector in the theory of free electrons, $\mathbf{r}$ is a position vector, and $u_{n \mathbf{k}}(\mathbf{r})$ is a periodic function that satisfies the boundary condition, $u_{n \mathbf{k}}(\mathbf{r})=u_{n \mathbf{k}}(\mathbf{r}+\mathbf{R})$. The corresponding energy eigenvalue is

$$
\epsilon_{n}(\mathbf{k})=\epsilon_{n}(\mathbf{k}+\mathbf{K}),
$$

where $\mathbf{K}$ is a reciprocal lattice vector. $\epsilon_{n}(\mathbf{k})$ is a continuous function, and since the energies associated with the index, $n$, vary with wave vector, $\mathbf{k}$, we speak of an energy band. All distinct values of $\epsilon_{n}(\mathbf{k})$ are represented by $\mathbf{k}$ values within the first Brillouin zone of the reciprocal lattice.

\subsubsection{Sampling of Brillouin Zone}

In principle, an infinite number of $\mathbf{k}$-points are necessary to account for infinite number of electrons in a periodic system. Bloch's theorem (Section 2.6.1) reduces the problem of calculating an infinite number of electronic wave functions to a finite number of wave functions for an infinite number of $\mathbf{k}$-points. In practice, one does not need an infinite number of $\mathbf{k}$-points since the electronic wave functions will be almost identical for $\mathbf{k}$-points that are very close to each other. Therefore, a single $\mathbf{k}$-point will be sufficient to represent the wave functions over a particular segment of $\mathbf{k}$-space. In general, structures with high symmetry can be sampled with a reduced k-point set, since it is sufficient to consider the $\mathbf{k}$-points only within the irreducible part of the Brillouin zone. Metallic systems require dense set of $\mathbf{k}$-points for a more accurate determination of Fermi level. There exist several methods to generate suitable kpoint sets and corresponding weights 20 . Using these $\mathbf{k}$-point sets generated by such methods, an accurate approximation of the electronic potential and total energy can be possible at a reduced computational cost. The magnitude of the errors in the calculated results decreases by making use of denser k-point sets.

\subsubsection{Plane wave Basis}

Using Bloch's theorem explained in Section 2.6.1, one electron wave functions at each k-point can be expanded in terms of discrete plane-waves. In principle, an infinite number of plane-waves are required to perform such an expansion. However, a finite number of plane-waves are sufficient to span the lowest energy electronic states, in which all the electrons of the physical system can be accommodated. Therefore, in practice, the number of plane-waves used in a calculation is determined by $\sqrt{E_{\text {cutoff }} 2 m_{e}} / \hbar$, where $E_{\text {cutoff }}$ is the kinetic cutoff energy. It is the only parameter that controls the accuracy of the plane wave basis set.

Plane wave basis sets are mathematically simple, cover all the space equally without being biased to any particular region, and span the Hilbert space completely. In this sense, they are superior to local basis sets, especially when the initial form of the 


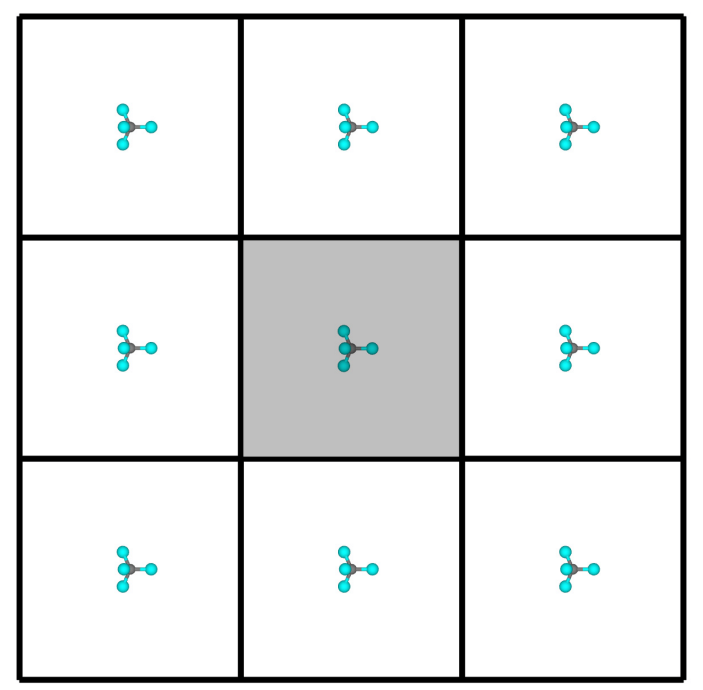

Figure 2.1: Schematic representation of a methane $\left(\mathrm{CH}_{4}\right)$ molecule placed in a cubic box of $20 \AA$ that obeys periodic boundary conditions. Calculations are only performed using the shaded supercell, and the rest of the images are due to periodicity.

wave functions is unknown. The main disadvantage of a plane wave basis compared to local basis sets is the computational costs.

\subsubsection{Non-periodic Systems}

In real life, the periodicity of the systems can be hampered by defects in the crystal structure. Bloch's description of waves, however, is only applicable to periodic systems. In order to be able to write the eigenfunctions in form of Eq. 2.17 the periodicity of the system need to be recovered. A practical approach to the problem is to use supercells in stead of unit cells. The supercells need to be chosen in a way to recover the periodicity of the system so that the Bloch's theorem can be applied. In some cases, it may be necessary to use very large supercells to perform the simulations. In this manner, even molecules can be studied by constructing supercells large enough to prevent the interactions between the molecules in neighboring cells. In Fig. 2.1 a cubic supercell with an edge length of $20 \AA$ is chosen to perform calculations on a single methane molecule $\left(\mathrm{CH}_{4}\right)$. Note that the image is repeated infinitely in three dimensions due to periodicity. However, the interaction of neighboring methane molecules is almost negligible at such distances. Naturally, large molecules require large supercells to maintain a sufficiently large separation between neighboring images. 


\subsection{Numerical Calculations}

The results presented in this thesis are obtained via first-principles plane wave calculations within density functional theory (DFT) as implemented in the Vienna $a b$ initio simulation package (VASP) $[21,22$. The kinetic cutoff energies for the plane-waves are chosen in a way as to assure a high convergence on the total energy, usually in the order of $10^{-3} \mathrm{eV} /$ atom. The cutoff energies are determined through initial test calculations, and the numerical values range from $400 \mathrm{eV}$ to $650 \mathrm{eV}$ depending on the chemical elements present. The Brillouin zone (BZ) is integrated using regular k-point grids 20. The Projector Augmented Wave (PAW) potentials are used to represent both the core and valence electrons [16, 17. The reliability of these PAW potentials is checked by verifying well-known experimental results, such as lattice parameters and cohesive energies of simple bulk materials. Exchangecorrelation potentials are approximated by the generalized gradient approximation (GGA) method using the Perdew Wang 91 (PW91) functional 8]. Some of the results are checked by using the Perdew Bucke Enzenhof (PBE) functional [9, 10. Geometries are optimized with or without symmetry constraints, using the conjugate gradient (CG) algorithm. During the geometry relaxations the partial occupancies of the wave functions for metallic systems are represented by smearing methods, such as gaussian or Methfessel-Paxton 23. The width of the smearing is carefully chosen to obtain a meaningful description of the energy. The numerical values for the smearing width are usually between $0.01 \mathrm{eV}$ and $0.1 \mathrm{eV}$. During the relaxation processes special care is taken to achieve a high electronic convergence, in the order of $10^{-4} \mathrm{eV}$ difference between two consecutive steps, in order to obtain accurate forces. A break condition of at most $0.02 \mathrm{eV} / \AA$ on total remaining force on each atom is set for the relaxation. The final total energies and charge densities of the optimized structures are obtained using the corrected linear tetrahedron method 24]. Further analysis of the densities of states, band structures and charge decompositions are performed on the fully optimized systems. A single $\mathrm{H}_{2}$ molecule is treated within a cubic box with a cell parameter of $10 \AA$, using periodic boundary conditions and $\Gamma$-point sampling, whereas other molecules are studied in a larger cubic box with a cell parameter of $20 \AA$. For relaxations in slab calculations a spacing of approximately $\sim 20 \AA$ between the slabs is conserved to prevent interactions of repeated periodic images.

\subsubsection{Convergence Tests}

The choice of pseudopotential, cutoff energy for the plane wave basis, and $\mathbf{k}$-points to span the Brillouin zone (BZ), are the three most important factors that determine the quality of the numerical calculations. As mentioned in Section 2.5.1, the PAW potentials provide accuracy at a reasonable computational effort. However, there is no global value for the plane wave cutoff energy or a set of $\mathbf{k}$-points that works fine for every individual system. Reliable results can only be obtained if accurate cutoffs $\left(E_{\text {cutoff }}\right)$ and dense $\mathbf{k}$-points are used. $E_{\text {cutoff }}$ purely depends on the chemical 

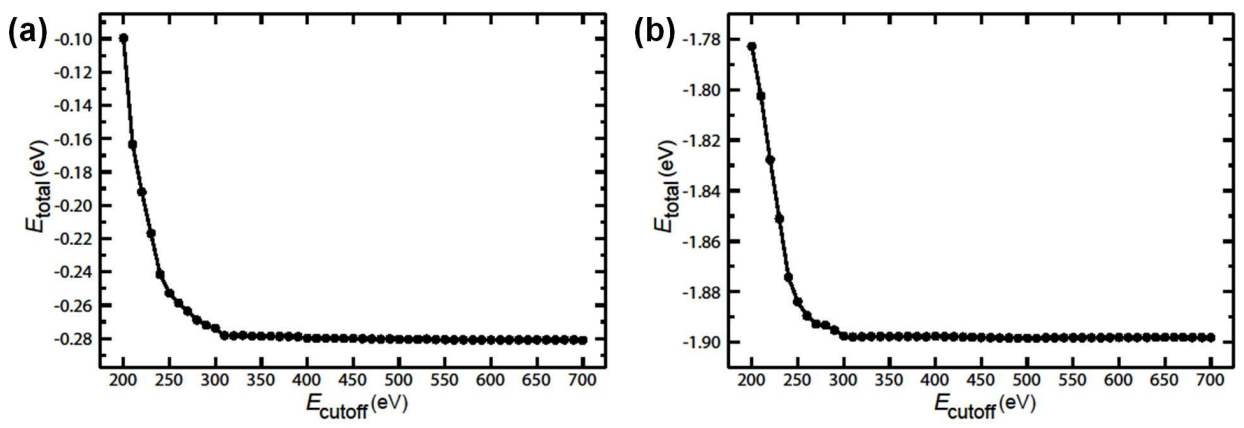

Figure 2.2: The variation of total energies w.r.t. $E_{\text {cutoff }}$ used in calculation for $\mathrm{Li}$ in atomic form (a), and bulk form (b). The energies are given in units of electron volt and the calculated total energies are per Li.

elements used in the calculations.

If the material of interest is composed of several chemical elements, then it is appropriate to set $E_{\text {cutoff }}$ to that of the value of the element with the highest cutoff. As an example, Fig. 2.2 shows the calculated total energy values for Li in atomic form (a), and bulk form (b), as a function of $E_{\text {cutoff }}$. Obviously, the $E_{\text {cutoff }}$ need to be chosen from the region where the the variation in total energy is negligible while keeping in mind that higher cutoff values require more computational effort. For Li a value of $E_{\text {cutoff }}=320 \mathrm{eV}$ will be an adequate choice. A Similar analysis also needs to be performed for $\mathbf{k}$-point sampling. A system specific $\mathbf{k}$-point mesh should be dense enough the BZ to acquire the convergence in total energy as in the case of $E_{\text {cutoff }}$.

\subsubsection{Thermodynamics}

Thermodynamics is defined as the branch of science that deals with conversion of energy into work and heat and its relation to macroscopic variables such as temperature, volume and pressure. To address the thermodynamics of chemical processes, such as chemical reactions, one can make use of the mathematical methods of Josiah Willard Gibbs [25]. The Gibbs free energy of a system is defined as:

$$
G=H-T S,
$$

where $H$ is the enthalpy, $S$ is the entropy, and $T$ is the temperature in units of kelvin. The change in the free energy of the system that occurs during a reaction measures the balance between the enthalpy and the entropy. Under constant temperature and pressure, if the change in the Gibbs energy from state A to state B is negative, then state $\mathrm{B}$ is said to be thermodynamically more stable than state A. At $T=0 \mathrm{~K}$, the change in Gibbs energy equals the change in enthalpy, $\Delta G=\Delta H$. The change in enthalpy is defined as the change in the internal energy of the system, $\Delta U$, plus the work that the system has done on its surroundings, $P \Delta V$. Since moreover 
the change in $P V$ for reactions involving solids is negligible, mostly it is sufficient to focus upon the change in energy, $\Delta U$, where $U$ corresponds to the ground state energy of a system as calculated by DFT.

\subsection{2.a Zero-Point Energy Contribution: ZPE}

The lowest possible energy that a quantum mechanical physical system may have is called the zero-point energy (ZPE) $26-28$. It arises as a result of nuclear motions. In molecular systems, both the vibrational and the rotational motions contribute to the ZPE. In solids, only the vibrational motions contribute. Within the harmonic oscillator approximation, the contribution of vibrational energies to the total energy term is calculated using the following equation:

$$
E^{v i b}=\int\left\{\frac{\hbar \omega}{2}+\hbar \omega n(\omega)\right\} g(\omega) d \omega,
$$

where $g(\omega)$ is the phonon density of states, and $n(\omega)=\left[e^{\frac{\hbar \omega}{k_{B} T}}-1\right]^{-1}$ is the BoseEinstein occupation number. At zero Kelvin, the ZPE contribution to the total energy is given by the first term of Eq. 2.20 alone, whereas the second term represents the finite temperature contributions. Vibrational contributions to the entropy term can also be calculated when finite temperature results are demanded.

The numerical calculation of ZPE can be accomplished using a dynamical matrix which is constructed via small displacements of atoms from their equilibrium positions and finite differencing the accumulated forces. Each atom inside the cell is symmetrically displaced in its three degrees of freedom, by $d r= \pm 0.01 \AA$. To account for the spatial range of the dynamical matrix the calculations require large supercells. ZPE contributions to the calculated total energies are considerable for light elements such as hydrogen. For heavy elements the phonon frequencies are low and accordingly the ZPEs are negligible.

\subsubsection{Kinetics}

Thermodynamic calculations focus only on the initial and final states of a system and the path by which a change takes place is not taken into account. Intuitively, one might expect strongly exothermic reactions to occur spontaneously, but this is often not true, since many potentially favorable reactions are prohibited by substantial energy barriers. To have a better understanding of how chemical reactions take place, methods based on Transition State Theory (TST) 29] can be used. In principle, the transition rate between two stable equilibria is determined by the lowest energy barrier, also called the reaction barrier (RB). The nudged elastic band method (NEB), which is proposed by Jónsson and Henkelman [30-33], is one of the suitable ways to calculate the RBs. Once the transition states and the RBs are known, other important factors relevant to chemical kinetics, such as, diffusion rates $k$, and constants $D$, can be obtained from a vibrational analysis.

The set up of a NEB calculation starts with a discrete representation of a path from the initial reactant configuration to the final product configuration. The coordinates 
of atoms for the initial and final configurations are kept fixed during the calculation. Based on these two stable structures, several intermediate configurations (the so called images) are generated using a straight line interpolation. An optimization algorithm is then applied to these images, so that they are relaxed down towards the minimum energy path (MEP). A refinement of MEP and accordingly a more accurate value for the RB is obtained by using denser set of images during calculations. The details on theoretical background of the NEB method as well as its comparison to other saddle point searching methods can be obtained in Refs. [30-34]

\subsubsection{Density of States: DOS}

The number of states at each energy level that are available to be occupied is called the density of states (DOS). A zero DOS for an energy level means that no states can be occupied. On contrary, a high DOS for an energy level represents that many states are available for occupation. DOS delivers invaluable information about the bonding within solids and in classification of materials as metallic, semiconductor or insulators. Metals or semi metals have non-localized electrons and no gap (separation between valence and conduction band). Materials with a large gap $(\geq 4 \mathrm{eV})$ are called insulators, whereas systems with a smaller gap are categorized as semiconductors. In the representation of DOS the specific relation between $\epsilon$ and $\mathbf{k}$ must be known to convert between energy and wavevector [18, 19. In the case of a parabolic relation, such as applies to free electrons, or to electrons in a solid with an isotropic parabolic band structure, the energy is related to the wavevector as: $\epsilon=\frac{\hbar^{2}}{2 m} k^{2}$. Accordingly, the density of states in three dimensions is given by,

$$
D(\epsilon)=\frac{1}{2 \pi^{2}} \cdot\left(\frac{2 m}{\hbar^{2}}\right)^{3 / 2} \cdot \epsilon^{1 / 2} .
$$

Although DFT seems to systematically underestimate the band gap in insulators and semiconductors by about $30-40 \%$, it is successful in reproducing the shape of the DOS.

\subsubsection{Charge Analysis}

In order to make a quantitative conclusion, the identification of the amount of electrons on a particular atom would be useful. However, there is no unique way to extract the number of electrons that are associated with a particular atom in a molecule, or in a solid. Many different schemes have been proposed, some are based on population analysis of wave functions (Mulliken population analysis [35, Coulson's charges [36]), and some others are based on partitioning of electron density distributions (Bader analysis [37, Hirshfeld analysis [38]). The charge density analysis of the materials discussed in this thesis are performed via the Bader analysis [39, 40]. In this approach, molecules or solids are partitioned into atomic volumes, such that the flux of the gradient of the electron density vanishes at every point on the surfaces. That is for every point $\mathbf{r}_{s}$ on the surface $\mathrm{S}\left(\mathbf{r}_{s}\right)$, 


$$
\nabla n\left(\mathbf{r}_{s}\right) \cdot u\left(\mathbf{r}_{s}\right)=0
$$

where $u\left(\mathbf{r}_{s}\right)$ is the unit vector normal to the surface at $\mathbf{r}_{s}$. The charge density reaches a minimum between atoms, which defines a natural location to separate the atoms from each other. The electron density used in a Bader analysis is obtained via self consistent static DFT calculations based on the equilibrium structure.

\subsubsection{Electron Localization Function: ELF}

Introduced by Becke and Edgecombe 41, and later adapted to DFT by Savin [42, 43, the electron localization function (ELF) provides a topological description of chemical bonding. ELF is a measure of the likelihood of finding an electron in the neighborhood space of a reference electron with the same spin, located at a given point. It is applicable to a variety of systems including molecules and solids. In principle, an approximate extraction of the ELF is possible from the experimental electron density that is measured in accurate X-ray diffraction experiments [44, 45. However, it is more directly obtained from quantum mechanical calculations. Keeping in mind that the electron density is given by the single particle wave functions as shown in Eq. 2.6, Savin's interpretation the ELF is defined as:

$$
\mathrm{ELF}=\left\{\left[\frac{T(\mathbf{r})}{T_{h}(\mathbf{r})}\right]^{2}+1\right\}^{-1},
$$

where, $T(\mathbf{r})$ is the excess local kinetic energy due to Pauli exclusion principle,

$$
T(\mathbf{r})=\frac{1}{2} \sum_{i}\left|\nabla \Psi_{i}(\mathbf{r})\right|^{2}-\frac{|\nabla n(\mathbf{r})|^{2}}{8 n(\mathbf{r})},
$$

and $T_{h}(\mathbf{r})$ is the Thomas-Fermi kinetic energy density, such that

$$
T_{h}(\mathbf{r})=\frac{3}{10}\left(3 \pi^{2}\right)^{2 / 3} n(\mathbf{r})^{5 / 3}
$$

The numerical values of ELF vary between 0 and 1 , where the value 1 means perfect localization of electrons. A value of 0.5 corresponds to the electron gas. The most elegant way to analyze ELF is via a graphical representation in form of iso-surfaces and cut planes. Although the correlation between ELF and chemical bonding is sort of topological, ELF is found to be useful in distinguishing between ionic and covalent type of interactions in materials.

\section{Bibliography}

[1] M. L. Born and R. Oppenheimer, Ann. Phys. 84, 457 (1927).

[2] P. Hohenberg and W. Kohn, Phys. Rev. 136, B864 (1964). 
[3] L. H. Thomas, Proc. Cambridge Philos. Soc. 23, 542 (1927).

[4] E. Fermi, Z. Phys. 48, 37 (1928).

[5] W. Kohn and L. J. Sham, Phys. Rev. 140, A1133 (1965).

[6] D. M. Ceperley and B. J. Alder, Phys. Rev. Lett. 45, 566 (1980).

[7] J. P. Perdew and A. Zunger, Phys. Rev. B 23, 5048 (1981).

[8] J. P. Perdew et al., Phys. Rev. B 46, 6671 (1992).

[9] J. P. Perdew, K. Burke, and M. Ernzerhof, Phys. Rev. Lett. 77, 3865 (1996).

[10] J. P. Perdew, K. Burke, and M. Ernzerhof, Phys. Rev. Lett. 78, 1396 (1997).

[11] D. R. Hamann, M. Schlüter, and C. Chiang, Phys. Rev. Lett. 43, 1494 (1979).

[12] G. B. Bachelet, D. R. Hamann, and M. Schlüter, Phys. Rev. B 26, 4199 (1982).

[13] N. Troullier and J. L. Martins, Phys. Rev. B 43, 1993 (1991).

[14] D. Vanderbilt, Phys. Rev. B 41, 7892 (1990).

[15] K. Laasonen, A. Pasquarello, R. Car, C. Lee, and D. Vanderbilt, Phys. Rev. B 47, 10142 (1993).

[16] P. E. Blöchl, Phys. Rev. B 50, 17953 (1994).

[17] G. Kresse and D. Joubert, Phys. Rev. B 59, 1758 (1999).

[18] N. W. Ashcroft and N. D. Mermin, Solid State Physics (Thomson Learning, Inc., 1976).

[19] C. Kittel, Introduction to Solid State Physics (New York: John Wiley \& Sons, Inc., 1996).

[20] H. J. Monkhorst and J. D. Pack, Phys. Rev. B 13, 5188 (1976).

[21] G. Kresse and J. Hafner, Phys. Rev. B 47, 558 (1993).

[22] G. Kresse and J. Furthmüller, Phys. Rev. B 54, 11169 (1996).

[23] M. Methfessel and A. T. Paxton, Phys. Rev. B 40, 3616 (1989).

[24] P. E. Blöchl, O. Jepsen, and O. K. Andersen, Phys. Rev. B 49, 16223 (1994).

[25] J. W. Gibbs, Elementary Principles in Statistical Mechanics (C. Scribner's sons, 1902).

[26] A. Einstein and L. Hopf, Ann. Phys. 33, 1096 (1910).

[27] A. Einstein and L. Hopf, Ann. Phys. 33, 1005 (1910). 
[28] A. Einstein and O. Stern, Ann. Phys. 40, 551 (1913).

[29] P. Atkins and J. de Paula, Atkins' Physical Chemistry (New York: Oxford University Press Inc., 2002).

[30] H. Jónsson, G. Mills, and K. W. Jacobsen, Nudged Elastic Band Method for Finding Minimum Energy Paths of Transitions, in Classical and Quantum Dynamics in Condensed Phase Simulations (Singapore: World Scientific, 1998).

[31] G. Henkelman and H. Jónsson, J. Chem. Phys. 113, 9978 (2000).

[32] G. Henkelman, B. P. Uberuaga, and H. Jónsson, J. Chem. Phys. 113, 9901 (2000).

[33] D. Sheppard, R. Terrell, and G. Henkelman, J. Chem. Phys. 128, 134106 (2008).

[34] R. A. Olsen, G. J. Kroes, G. Henkelman, A. Arnaldsson, and H. Jónsson, J. Chem. Phys. 121, 9776 (2004).

[35] R. S. Mulliken, J. Chem. Phys. 23, 1833 (1955).

[36] J. Meister and W. H. E. Schwarz, J. Phys. Chem. 98, 8245 (1994).

[37] R. Bader, Atoms in Molecules: A Quantum Theory. (USA: Oxford University Press, 1994).

[38] F. L. Hirshfeld, Theor. Chim. Acta 44, 129 (1977).

[39] G. Henkelman, A. Arnaldsson, and H. Jónsson, Comput. Mater. Sci. 36, 354 (2006).

[40] E. Sanville, S. D. Kenny, R. Smith, and G. Henkelman, J. Comput. Chem. 28, 899 (2007).

[41] A. D. Becke and K. E. Edgecombe, J. Chem. Phys. 92, 5397 (1990).

[42] M. Kohout and A. Savin, J. Comput. Chem. 18, 1431 (1997).

[43] A. Savin, THEOCHEM 727, 127 (2005).

[44] W. H. E. Schwarz and B. Müller, Chem. Phys. Lett. 166, 621 (1990).

[45] V. Tsirelsos and A. Stash, Chem. Phys. Lett. 351, 142 (2002). 
Part - A

Atomic Hydrogen Storage 



\section{Chapter 3}

\section{Hydrogen storage in magnesium-transition metal compounds}

\subsection{Abstract}

Magnesium-dihydride $\left(\mathrm{MgH}_{2}\right)$ stores 7.7 weight \% hydrogen, but it suffers from a high thermodynamic stability and slow (de)hydrogenation kinetics. Alloying Mg with lightweight transition metals $(\mathrm{TM}=\mathrm{Sc}, \mathrm{Ti}, \mathrm{V}, \mathrm{Cr})$ aims at improving the thermodynamic and kinetic properties. We study the structure and stability of $\mathrm{Mg}_{x} \mathrm{TM}_{1-x} \mathrm{H}_{2}$ compounds, $x=[0-1]$, by first-principles calculations at the level of density functional theory. We find that the experimentally observed sharp decrease in hydrogenation rates for $x \gtrsim 0.8$ correlates with a phase transition of $\mathrm{Mg}_{x} \mathrm{TM}_{1-x} \mathrm{H}_{2}$ from a fluorite to a rutile phase. The stability of these compounds decreases along the series Sc, $\mathrm{Ti}, \mathrm{V}, \mathrm{Cr}$. Varying the transition metal (TM) and the composition $x$, the formation enthalpy of $\mathrm{Mg}_{x} \mathrm{TM}_{1-x} \mathrm{H}_{2}$ can be tuned over the substantial range $0-2 \mathrm{eV} /$ f.u. Assuming however that the alloy $\mathrm{Mg}_{x} \mathrm{TM}_{1-x}$ does not decompose upon dehydrogenation, the enthalpy associated with reversible hydrogenation of compounds with a high magnesium content $(x=0.75)$ is close to that of pure $\mathrm{Mg}$.

\subsection{Introduction}

Hydrogen is a clean energy carrier and an alternative to carbon based fuels in the long run. [1] Mobile applications require a compact, dense and safe storage 
of hydrogen with a high-rate loading and unloading capability. [2, 3] Lightweight metal hydrides could satisfy these requirements. 4, 5] Metal hydrides are formed by binding hydrogen atoms in the crystal lattice, resulting in very high volumetric densities. Reasonable hydrogen gravimetric densities in metal hydrides can be achieved if lightweight metals are used.

$\mathrm{MgH}_{2}$ has been studied intensively since it has a relatively high hydrogen gravimetric density of 7.7 wt. \%. Bottlenecks in the application of $\mathrm{MgH}_{2}$ are its thermodynamic stability and slow (de)hydrogenation kinetics. These lead to excessively high operating temperatures $(573-673 \mathrm{~K})$ for hydrogen release. [6 8 ] The hydrogen (de)sorption rates can be improved by decreasing the particle size down to nanoscales. [9-11] It is predicted that particles smaller than $1 \mathrm{~nm}$ have a markedly decreased hydrogen desorption enthalpy, which would lower the operating temperature.[12] The production of such small particles is nontrivial, however, and the hydrogen (de)sorption rates of larger nanoparticles are still too low.

An additional way of improving the (de)hydrogenation kinetics of $\mathrm{MgH}_{2}$ is to add transition metals (TMs). [9, 13-15. Usually only a few wt. \% is added, since TMs are thought to act as catalysts for the dissociation of hydrogen molecules. Recently however, Notten and co-workers have shown that the (de)hydrogenation kinetics is markedly improved by adding more TM and making alloys $\mathrm{Mg}_{x} \mathrm{TM}_{(1-x)}, \mathrm{TM}=\mathrm{Sc}$, Ti, $x \lesssim 0.8$. [16 26. The basic ansatz is that the rutile crystal structure of $\mathrm{MgH}_{2}$ enforces an unfavorably slow diffusion of hydrogen atoms. 27. $\mathrm{ScH}_{2}$ and $\mathrm{TiH}_{2}$ have a fluorite structure, which would be more favorable for fast hydrogen kinetics. By adding a sufficiently large fraction of these TMs one could force the $\operatorname{Mg}_{x} \mathrm{TM}_{(1-x)}$ compound to adopt the fluorite structure.

In this paper we examine the structure and stability of $\mathrm{Mg}_{x} \mathrm{TM}_{(1-x)} \mathrm{H}_{2}, \mathrm{TM}=$ $\mathrm{Sc}, \mathrm{Ti}, \mathrm{V}, \mathrm{Cr}$, compounds by first-principles calculations. In particular, we study the relative stability of the rutile versus the fluorite structures. This paper is organized as follows. In Sec. 3.3 we discuss the computational details. The calculations are benchmarked on the $\mathrm{TMH}_{2}$ simple hydrides. The structure and formation enthalpies of the compounds $\mathrm{Mg}_{x} \mathrm{TM}_{(1-x)} \mathrm{H}_{2}$ are studied in Sec. 3.4 and an analysis of the electronic structure is given. We discuss the hydrogenation enthalpy of the compounds in Sec. 3.5 and summarize our main results in Sec. 3.6 .

\subsection{Computational Methods and Test Calculations}

We perform first-principles calculations at the level of density functional theory (DFT) with the PW91 functional as the generalized gradient approximation (GGA) to exchange and correlation. 28] As transition metals have partially filled $3 d$ shells we include spin polarization and study ferromagnetic and simple antiferromagnetic orderings where appropriate. A plane wave basis set and the projector augmented wave (PAW) formalism are used, [29, 30] as implemented in the VASP code. [31, 32. The cutoff kinetic energy for the plane waves is set at $650 \mathrm{eV}$. The total energies 
of $\mathrm{MgH}_{2}, \mathrm{TMH}_{2}$ and the hydrogen molecule are then converged to within $1 \mathrm{meV}$. Standard frozen core potentials are applied for all the elements, except for Sc, where we include $3 s$ and $3 p$ as valence shells, in addition to the usual $4 s$ and $3 d$ shells. The Brillouin zone (BZ) is integrated using a regular $\mathbf{k}$-point mesh with a spacing $\sim 0.02 \AA^{-1}$ and the Methfessel-Paxton scheme with a smearing parameter of $0.1 \mathrm{eV}$. 33. The self-consistency convergence criterion for the energy difference between two consecutive electronic steps is set to $10^{-5} \mathrm{eV}$. Structural optimization is assumed to be complete when the total force acting on each atom is smaller than $0.01 \mathrm{eV} / \AA$. The volumes of the unit cells are relaxed, and, where appropriate, also their shapes. Finally, we calculate accurate total energies for the optimized geometries using the linear tetrahedron method. 34.

To calculate the formation enthalpies of the metal hydrides we consider the following reaction.

$$
x \mathrm{Mg}+(1-x) \mathrm{TM}+\mathrm{H}_{2}(g) \longrightarrow \mathrm{Mg}_{x} \mathrm{TM}_{(1-x)} \mathrm{H}_{2} .
$$

The formation enthalpies (at $T=0$ ) are then obtained by subtracting the total energies of the reactants from that of the product. A cubic box of size $10 \AA$ is applied for the $\mathrm{H}_{2}$ molecule. The calculated $\mathrm{H}-\mathrm{H}$ bond length, binding energy, and vibrational frequency are $0.748 \AA,-4.56 \mathrm{eV}$ and $4351 \mathrm{~cm}^{-1}$, respectively, in good agreement with the experimental values of $0.741 \AA,-4.48 \mathrm{eV}$ and $4401 \mathrm{~cm}^{-1}$. [35, 36.

Since hydrogen is a light element, the zero point energy (ZPE) due to its quantum motion, is not negligible. We find that the correction to the reaction enthalpies of Eq. (3.1) resulting from the ZPEs, is $0.15 \pm 0.05 \mathrm{eV} / \mathrm{H}_{2}$, as function of the composition $x$ and the transition metal TM. Since we are mainly interested in relative formation enthalpies, we omit the ZPE energy correction in the following.

Before discussing the $\mathrm{Mg}_{x} \mathrm{TM}_{(1-x)} \mathrm{H}_{2}$ compounds we benchmark our calculations on the simple compounds $\mathrm{MgH}_{2}$ and $\mathrm{TMH}_{2}$. Under standard conditions magnesiumdihydride has the rutile structure, $\alpha-\mathrm{MgH}_{2}$, see Fig. 4.1, with space group $\mathrm{P} 4_{2} / \mathrm{mnm}$ (136) and $\mathrm{Mg}$ and $\mathrm{H}$ atoms in the $2 a$ and $4 f(x=0.304)$ Wyckoff positions, respectively. Each $\mathrm{Mg}$ atom is coordinated octahedrally by $\mathrm{H}$ atoms, with two $\mathrm{Mg}-\mathrm{H}$ distances of $1.94 \AA$ and four distances of $1.95 \AA$. First row early transition metal hydrides crystallize in the fluorite structure, $\alpha-\mathrm{TMH}_{2}$, see Fig. 4.1, with space group $\mathrm{Fm} \overline{3} \mathrm{~m}(225)$ and TM atoms in $4 a$ and $\mathrm{H}$ atoms in $8 c$ Wyckoff positions. Each TM has a cubic surrounding of $\mathrm{H}$ atoms with calculated TM-H bond lengths of 2.07, 1.92, 1.82 and $1.79 \AA$ for Sc, Ti, V, and Cr, respectively. By breaking the cubic symmetry by hand and reoptimizing the geometry we have confirmed that the fluorite structure indeed represents a stable minimum. 

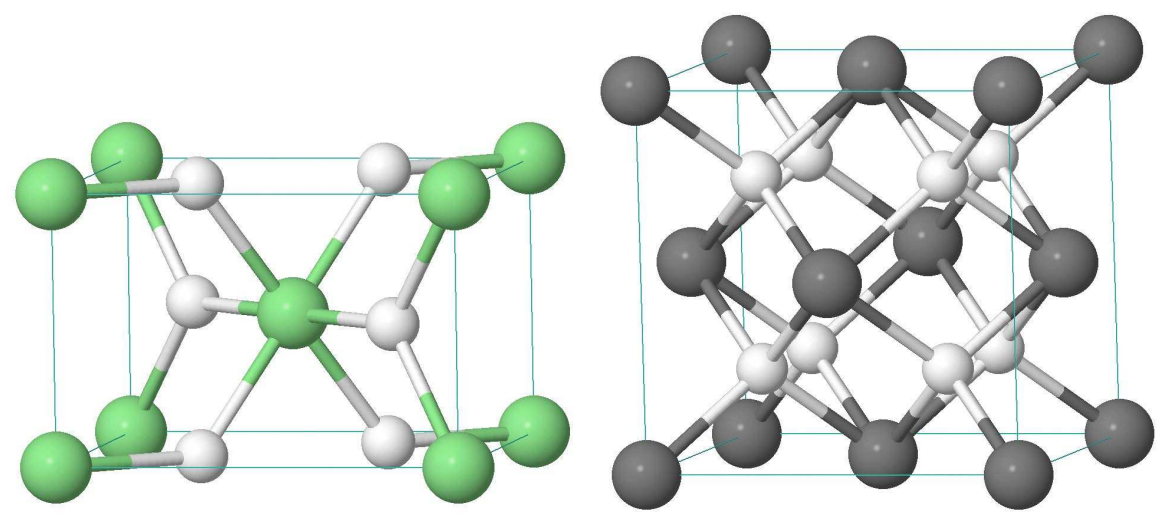

Figure 3.1: (Left) rutile crystal structure of $\alpha-\mathrm{MgH}_{2}$, and (right) fluorite crystal structure of $\alpha-\mathrm{TMH}_{2}$. The white spheres represent the hydrogen atoms.

The optimized cell parameters and the calculated formation enthalpies of the simple hydrides are given in Table 3.1. The structural parameters are in good agreement both with available experimental data and with previous DFT calculations. 37 39 The formation enthalpies of $\mathrm{MgH}_{2}$ and $\mathrm{VH}_{2}$ are somewhat underestimated by the calculations, whereas those of $\mathrm{ScH}_{2}$ and $\mathrm{TiH}_{2}$ are in excellent agreement with experiment. $\mathrm{CrH}_{2}$ is predicted to be unstable with respect to decomposition.

\subsection{Results $\mathrm{Mg}_{x} \mathrm{TM}_{(1-x)} \mathrm{H}_{2}$}

\subsubsection{Structures and formation enthalpies}

$\mathrm{Mg}_{x} \mathrm{TM}_{(1-x)} \mathrm{H}_{2}$ has the fluorite structure for $x=0$, and the rutile structure for $x=1$. We want to establish which of the two structures is most stable at intermediate compositions $x$. First we summarize the current status of the experimental work on $\mathrm{Mg}_{x} \mathrm{TM}_{(1-x)}$ alloys.

Experimentally it has been demonstrated that $\mathrm{Mg}_{x} \mathrm{Sc}_{(1-x)}$ alloys can be reversibly hydrogenated, both in thin films, as well as in bulk form. [16, 17, 19, 20, 44, 45] $\mathrm{Mg}$ and $\mathrm{Ti}$ do not form a stable bulk alloy, but thin films of $\mathrm{Mg}_{x} \mathrm{Ti}_{(1-x)}$ have been made, which are readily and reversibly hydrogenated. [21 26, 46] Thin films of $\mathrm{Mg}_{x} \mathrm{~V}_{(1-x)}$ and $\mathrm{Mg}_{x} \mathrm{Cr}_{(1-x)}$ can also be easily hydrogenated. [17] Attempts to produce nonequilibrium bulk $\mathrm{Mg}_{x} \mathrm{Ti}_{(1-x)}$ alloys by ball milling of $\mathrm{Mg}$ and $\mathrm{Ti}$ or their hydrides have had a limited success so far. [47 50. However, $\mathrm{Mg}_{7} \mathrm{TiH}_{y}$ crystals have been made using a high pressure anvil technique. [51] The same technique has been applied to 
Table 3.1: Optimized cell parameters $a(c)$, and calculated formation enthalpies $E_{f}$, of elemental dihydrides in their most stable $(\alpha)$ forms. All $\mathrm{TMH}_{2}$ have a fluorite structure, space group $\mathrm{Fm} \overline{3} \mathrm{~m}$ (225), whereas $\mathrm{MgH}_{2}$ has a rutile structure, space group $\mathrm{P} 4_{2} / \mathrm{mnm}$ (136).

\begin{tabular}{|c|c|c|c|c|}
\hline \multirow[b]{2}{*}{ Compound } & \multicolumn{2}{|c|}{$\begin{array}{l}a(c) \\
(\AA)\end{array}$} & \multicolumn{2}{|c|}{$\begin{array}{c}E_{f} \\
\text { (eV/f.u.) }\end{array}$} \\
\hline & Calc & Exp & Calc & Exp \\
\hline $\mathrm{MgH}_{2}$ & $4.494(3.005)$ & $4.501(3.010)^{\mathrm{a}}$ & -0.66 & -0.76 \\
\hline $\mathrm{ScH}_{2}$ & 4.775 & $4.78^{\mathrm{b}}$ & -2.09 & -2.08 \\
\hline $\mathrm{TiH}_{2}$ & 4.424 & $4.454^{\mathrm{c}}$ & -1.47 & -1.45 \\
\hline $\mathrm{VH}_{2}$ & 4.210 & $4.27^{\mathrm{d}}$ & -0.65 & -0.79 \\
\hline $\mathrm{CrH}_{2}$ & 4.140 & $3.861^{\mathrm{d}}$ & $+0.13^{\mathrm{e}}$ & - \\
\hline
\end{tabular}

a Reference 40$]$
beference $[41]$
' $R$ Reference 42$]$
${ }^{\text {d }}$ Reference $[43]$
e Antiferromagneticly ordered.

produce the hydrides $\mathrm{Mg}_{6} \mathrm{VH}_{y}$ and $\mathrm{Mg}_{3} \mathrm{CrH}_{y}$. 52 54.

The crystal structure of $\mathrm{Mg}_{x} \mathrm{Sc}_{(1-x)} \mathrm{H}_{y}$ and $\mathrm{Mg}_{x} \mathrm{Ti}_{(1-x)} \mathrm{H}_{y}$ in thin films, $x \lesssim 0.8$, $y \approx 1-2$, is cubic, with the $\mathrm{Mg}$ and $\mathrm{TM}$ atoms at fcc positions. No detectable regular ordering of $\mathrm{Mg}$ and $\mathrm{TM}$ atoms at these positions has been found. 23, 44, 45. In contrast, the $\mathrm{Mg}$ and $\mathrm{TM}$ atoms form simple ordered structures in the high pressure phases. [51 55] The hydrogen atoms in $\mathrm{Mg}_{0.65} \mathrm{Sc}_{0.35} \mathrm{H}_{y}, y \approx 1-2$, assume tetrahedral interstitial positions, as is expected for the fluorite structure. [44, 45]. In the $\mathrm{Mg}_{7} \mathrm{TiH}_{16}$ high pressure phase the metal atoms are in fcc positions and are ordered as in the $\mathrm{Ca}_{7} \mathrm{Ge}$ structure. [51. The $\mathrm{H}$ atoms are in interstitial sites, but displaced from their ideal tetrahedral positions. 55].

The latter structure can be used as a starting point to construct simple, fluoritetype structures for $\mathrm{Mg}_{x} \mathrm{TM}_{(1-x)} \mathrm{H}_{2}, 0<x<1$. For $x=0.125,0.875$ we use the $\mathrm{Ca}_{7} \mathrm{Ge}$ structure to order the metal atoms, for $x=0.25,0.75$ the $\mathrm{Cu}_{3} \mathrm{Au}\left(L 1_{2}\right)$ structure, and for $x=0.5$ the $\mathrm{CuAu}\left(L 1_{0}\right)$ structure. The $\mathrm{H}$ atoms are placed at or close to tetrahedral interstitial positions. As the atomic volumes of the various TM atoms differ, in each of the structures and compositions the cell parameters are optimized, as well as the positions of all atoms within the cell. Care is taken to allow for breaking the symmetry in the atomic positions. In particular the hydrogen atoms 
are often displaced from their ideal tetrahedral positions. Although these structures are then no longer ideal fluorite structures anymore, we still use the term fluorite in the following. To model $\mathrm{Mg}_{x} \mathrm{TM}_{(1-x)} \mathrm{H}_{2}$ in rutile-type structures we use the $\alpha-\mathrm{MgH}_{2}$ structure as a starting point. We replace a fraction $x$ of the $\mathrm{Mg}$ atoms by TM atoms and use the smallest supercell of the rutile structure where this leads to an integer number of atoms. In case of multiple possible cells, the results given below refer to the cell that leads to the lowest energy. Again we allow for symmetry breaking and optimize the cell parameters and the atomic positions.

To asses the effect of disorder in the positions of the metal atoms we performed test calculations on special quasi-random structures of $\operatorname{Mg}_{x} \operatorname{Ti}_{(1-x)} \mathrm{H}_{2}$, which enable to model random alloys in a finite supercell. For instance, we use a 32 atom supercell to model quasi-random structures of fcc $\mathrm{Mg}_{x} \mathrm{Ti}_{1-x}$ for $x=0.25,0.5$, and 0.75 , and a 64 atom supercell for $x=0.125$ and 0.875 . 56. Inserting hydrogen atoms in tetrahedral interstitial positions then gives supercells with a total number of atoms of 96 and 192, respectively. The cell parameters are optimized, as well as the atomic positions. [57. The formation energies according to Eq. (3.1) of $\mathrm{Mg}_{x} \mathrm{Ti}_{(1-x)} \mathrm{H}_{2}$ in these quasi-random structures, are within $\sim 0.1 \mathrm{eV} /$ f.u. lower than those of the simple ordered structures. In particular, the relative stability of the fluorite vs. the rutile structures is not sensitive to the relative ordering of the metal atoms. It is reasonable to expect that this also holds for the other $\mathrm{Mg}_{x} \mathrm{TM}_{(1-x)} \mathrm{H}_{2}$ compounds.

The calculated volumes $V\left(\mathrm{Mg}_{x} \mathrm{TM}_{(1-x)} \mathrm{H}_{2}\right)$ of the (simple) fluorite structures, normalized per formula unit (f.u.), are shown in Fig. 3.2. The volumes of the quasirandom structures of $\mathrm{Mg}_{x} \mathrm{Ti}_{(1-x)} \mathrm{H}_{2}$ are within $1 \%$ of those of the simple structures. The volumes of a few bulk compounds and compositions can be extracted from experimental data, thus providing a check on the calculations. Interpolating the results in Ref. 44] gives $V\left(\mathrm{Mg}_{0.65} \mathrm{Sc}_{0.35} \mathrm{H}_{2}\right)=27.5 \AA^{3}$, whereas the interpolated calculated value from Fig. 3.2 is $26.9 \AA^{3}$. Ref. [55] gives $V\left(\mathrm{Mg}_{0.875} \mathrm{Ti}_{0.125} \mathrm{H}_{2}\right)=27.3 \AA^{3}$, compared to the calculated value $26.6 \AA^{3}$. These differences between experimental and calculated volumes are consistent with the differences between experimental and calculated lattice parameters of the simple hydrides, see Table 3.1. Experiments on thin films give $V\left(\mathrm{Mg}_{0.7} \mathrm{Ti}_{0.3} \mathrm{H}_{2}\right)=26.4 \AA^{3}$, if one assumes cubic symmetry. [23] The interpolated computational value is $24.4 \AA^{3}$. The difference between these values is not excessively large, but considering the smaller difference found for the bulk composition $\mathrm{Mg}_{0.875} \mathrm{Ti}_{0.125} \mathrm{H}_{2}$, it may suggest that the structure of thin films is slightly different from that of bulk.

The trends observed in Fig. 3.2 can be interpreted straightforwardly. The cell volumes of $\alpha-\mathrm{ScH}_{2}$ and the cubic $\beta-\mathrm{MgH}_{2}$ structure [58] are within $1.4 \%$ of one 


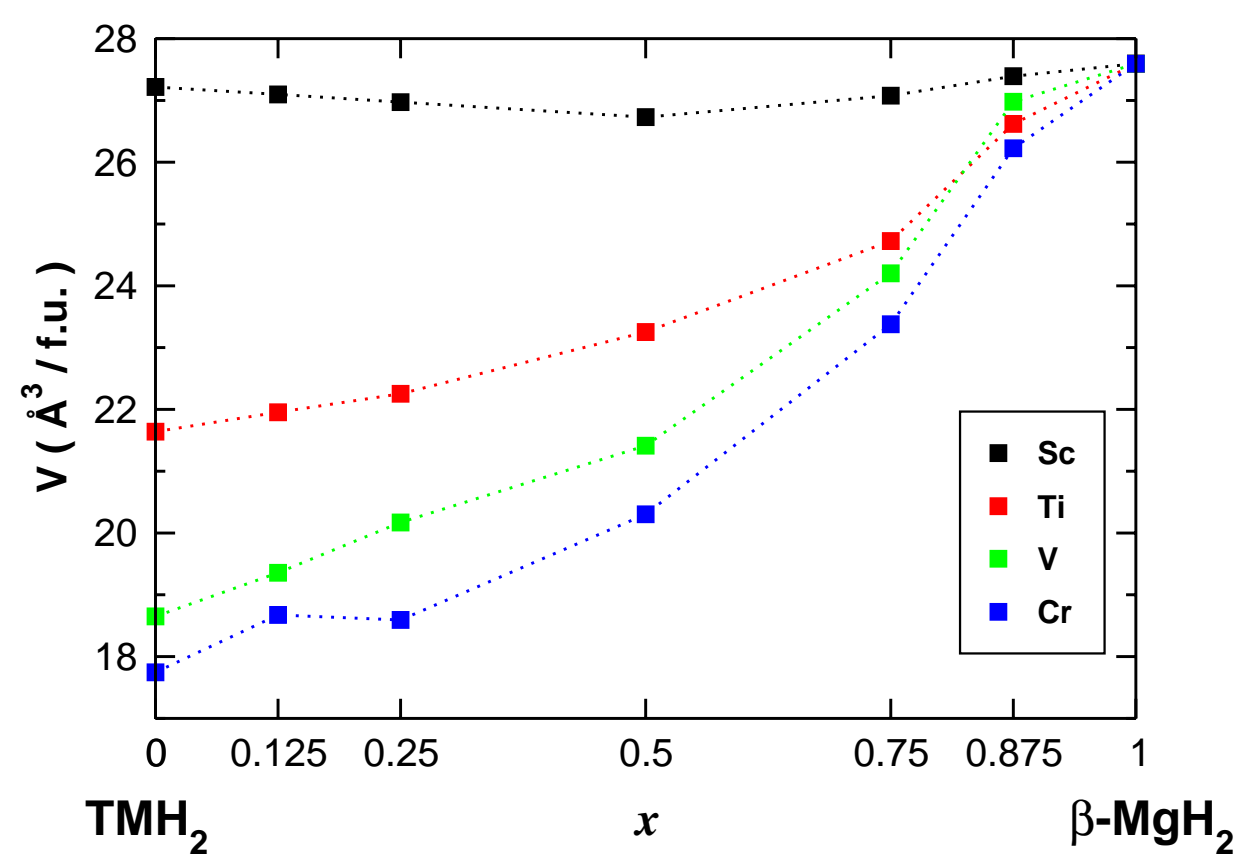

Figure 3.2: The volumes per formula unit in $\AA^{3}$ of $\mathrm{Mg}_{x} \mathrm{TM}_{(1-x)} \mathrm{H}_{2}$ in the fluorite structure, as a function of the composition $x$ for $\mathrm{TM}=\mathrm{Sc}, \mathrm{Ti}, \mathrm{V}, \mathrm{Cr}$ (from top to bottom).

another, which explains why the volumes calculated for $\mathrm{Mg}_{x} \mathrm{Sc}_{(1-x)} \mathrm{H}_{2}$ only weakly depend on the composition $x$. The cell volumes of the other $\mathrm{TMH}_{2}$ are smaller, hence one expects the volumes $V\left(\mathrm{Mg}_{x} \mathrm{TM}_{(1-x)} \mathrm{H}_{2}\right)$ to increase with $x$. At fixed composition $x$, the volumes $V\left(\mathrm{Mg}_{x} \mathrm{TM}_{(1-x)} \mathrm{H}_{2}\right)$ decrease along the series $\mathrm{Sc}, \mathrm{Ti}, \mathrm{V}$, $\mathrm{Cr}$, as the atomic volumes of the TMs decrease correspondingly. According to Zen's law of additive volumes one would expect [59.

$$
V\left(\mathrm{Mg}_{x} \mathrm{TM}_{(1-x)} \mathrm{H}_{2}\right)=x V\left(\beta-\mathrm{MgH}_{2}\right)+(1-x) V\left(\mathrm{TMH}_{2}\right) .
$$

The curves shown in Fig. 3.2 deviate slightly, but distinctly, from straight lines, with a maximum deviation of $\sim 5 \%$. This deviation is consistent with the experimental observations on $\mathrm{Mg}_{x} \mathrm{Ti}_{(1-x)} \mathrm{H}_{2}$. [21, 23] It is also observed in simple metal alloys. [59]

The calculated formation enthalpies of $\mathrm{Mg}_{x} \mathrm{TM}_{(1-x)} \mathrm{H}_{2}$ are shown in Fig. 4.6. 
Clearly for all TMs the fluorite structure is more stable than the rutile structure for all $x$ smaller than a critical value, $x_{c}$. The critical composition $x_{c}$ at which the rutile structure becomes more stable, as obtained by interpolation in Fig. 4.6, is in the range $x_{c} \approx 0.8-0.85$ for all TMs. The exact position of the critical composition $x_{c}$ depends somewhat on the details of the structure. For quasi-random structures of $\mathrm{Mg}_{x} \mathrm{Ti}_{(1-x)} \mathrm{H}_{2}$ the critical composition is at $x_{c}=0.875$. [57] The fact that the critical composition is fairly high might be guessed from the energies of the fluorite and rutile structures of the simple hydrides. As a first estimate of the critical composition $x_{c}$ below which the fluorite structure is stable one may try a linear interpolation between the pure $\mathrm{TMH}_{2}$ compounds, $x=0$, and $\mathrm{MgH}_{2}, x=1$. The rutile structure of $\mathrm{TMH}_{2}$ is more unstable than the fluorite structure by $\Delta\left(\mathrm{TMH}_{2}\right)=0.75,0.65$, $0.68,0.36 \mathrm{eV} / \mathrm{f} . \mathrm{u}$. for $\mathrm{TM}=\mathrm{Sc}, \mathrm{Ti}, \mathrm{V}, \mathrm{Cr}$, respectively. The difference in formation enthalpy between the $\alpha$ (rutile) and $\beta$ (cubic) phases of $\mathrm{MgH}_{2}$ is $\Delta\left(\mathrm{MgH}_{2}\right)=0.10$ $\mathrm{eV} /$ f.u. Linear interpolation then gives $x_{c}=\Delta\left(\mathrm{TMH}_{2}\right) /\left(\Delta\left(\mathrm{TMH}_{2}\right)+\Delta\left(\mathrm{MgH}_{2}\right)\right)$, which results in $x_{c} \approx 0.9$ for $\mathrm{Sc}, \mathrm{Ti}$ and $\mathrm{V}$, and $x_{c}=0.8$ for $\mathrm{Cr}$. Whereas these values may seem a good first guess, they are somewhat too high as compared to the crossing points $x_{c}$ observed in Fig. 4.6. Moreover, as this figure shows, in particular the curves for $\mathrm{Ti}$ and $\mathrm{V}$ are far from linear, so the results for the linear interpolation may be somewhat fortuitous.

Experimental results indicate that $\mathrm{Mg}_{0.7} \mathrm{Ti}_{0.3} \mathrm{H}_{2}$ has the fluorite structure and $\mathrm{Mg}_{0.9} \mathrm{Ti}_{0.1} \mathrm{H}_{2}$ has the rutile structure. [23. This agrees with the results shown in Fig. 4.1, where a fluorite to rutile phase transition takes place at the composition $x=$ $x_{c} \approx 0.83$. It has been suggested that the fluorite structure allows for a much faster kinetics of hydrogen loading and unloading. Experimentally it has been observed that the dehydrogenation kinetics of $\mathrm{Mg}_{x} \mathrm{Sc}_{(1-x)}$ and $\mathrm{Mg}_{x} \mathrm{Ti}_{(1-x)}$ becomes markedly slower if $x \gtrsim x_{0}=0.8$. The results shown in Fig. 4.1 suggest that $x_{0}=x_{c}$, i.e. the composition at which the phase transition between fluorite and rutile structures takes place.

The dehydrogenation kinetics of $\mathrm{Mg}_{x} \mathrm{Ti}_{(1-x)}$ also becomes gradually slower with decreasing $x$, for $x<x_{c}$, i.e. where the compound remains in the fluorite structure, although it is still faster than for $x>x_{c}$. [19, 21] Whereas kinetic studies are beyond the scope of the present paper, we speculate that a volume effect might play a role here. In the fluorite structure the hydrogen atoms occupy interstitial positions close to the tetrahedral sites. Diffusion of hydrogen atoms is likely to take place via other interstitial sites such as the octahedral sites. The smaller the volume, the shorter the distance between such sites and the occupied positions, or in other words, the shorter the distance between a diffusing hydrogen atom and other hydrogen atoms in 

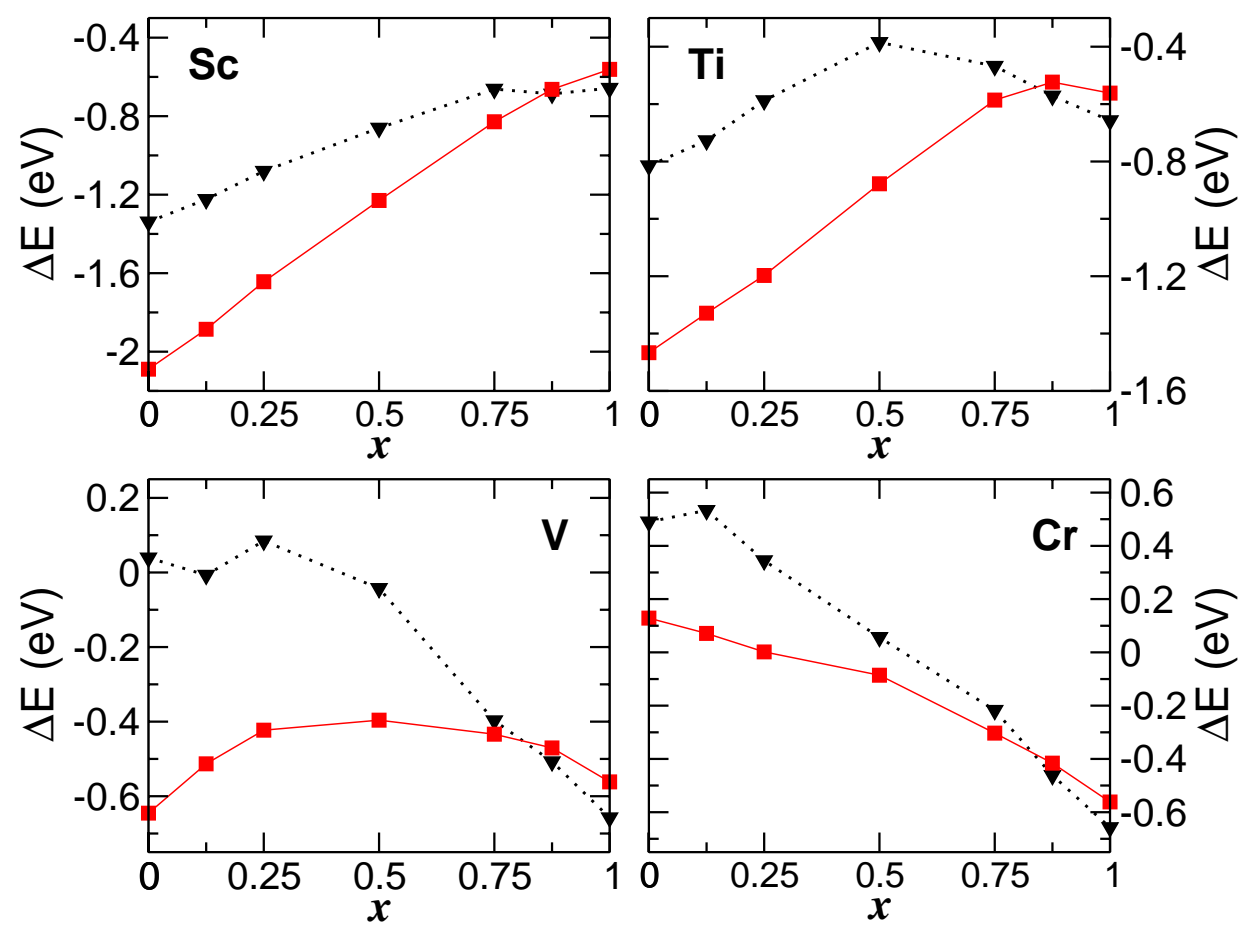

Figure 3.3: The formation enthalpy (per formula unit) of the $\mathrm{Mg}_{x} \mathrm{TM}_{(1-x)} \mathrm{H}_{2}$ compounds as obtained from spin polarized calculations. The values for the fluorite and rutile structures are represented by squares (solid lines) and triangles (dashed lines), respectively.

the lattice. This may increase the barrier for diffusion. As the volume of $\mathrm{Mg}_{x} \mathrm{~V}_{(1-x)}$ and $\mathrm{Mg}_{x} \mathrm{Cr}_{(1-x)}$ is smaller than that of $\mathrm{Mg}_{x} \mathrm{Sc}_{(1-x)}$ and $\mathrm{Mg}_{x} \mathrm{Ti}_{(1-x)}$ (at the same composition $x$ ), this might also explain why the dehydrogenation kinetics of the former compounds is much slower. 17]. We note that the smaller volume of $\mathrm{Mg}_{x} \mathrm{~V}_{(1-x)}$ and $\mathrm{Mg}_{x} \mathrm{Cr}_{(1-x)}$ is accompanied by a distortion of the structures consistent with the limited space available to accommodate the hydrogen atoms. For instance, in $\mathrm{Mg}_{0.75} \mathrm{~V}_{0.25} \mathrm{H}_{2}$ and $\mathrm{Mg}_{0.75} \mathrm{Cr}_{0.25} \mathrm{H}_{2}$ the hydrogen atoms are displaced considerably from the tetrahedral positions, and the coordination number of $\mathrm{V}$ and $\mathrm{Cr}$ (by hydrogen) is 7 , instead of 8 as in case of a perfect fluorite structure. 


\subsubsection{Electronic structure}

To analyze the electronic structure of the compounds $\mathrm{Mg}_{x} \mathrm{TM}_{(1-x)} \mathrm{H}_{2}$, we start with the density of states (DOS) of the pure hydrides $\alpha-\mathrm{MgH}_{2}$ and $\mathrm{TMH}_{2}$ as shown in Fig. 3.4. The bonding in $\mathrm{MgH}_{2}$ is dominantly ionic; occupied hydrogen orbitals give the main contribution to the valence states, whereas the conduction bands have a significant contribution from the Mg orbitals. 60. As usual, ionic bonding between main group elements results in an insulator with a large band gap. In contrast, the transition metal dihydrides are metallic, as demonstrated by Fig. 3.4. The peak in the DOS at low energy, i.e. between -9 and $-2 \mathrm{eV}$ in $\mathrm{ScH}_{2}$ to between -12 and $-4 \mathrm{eV}$ in $\mathrm{CrH}_{2}$, is dominated by hydrogen states. The broad peak around the Fermi level consists of transition metal $d$-states. It suggests that bonding in $\mathrm{TMH}_{2}$ is at least partially ionic. The TM $s$-electrons are transferred to the $\mathrm{H}$ atoms, whereas the $d$-electrons largely remain on the TM atoms. The DOSs of $\mathrm{TMH}_{2}, \mathrm{TM}=\mathrm{Sc}, \mathrm{Ti}, \mathrm{V}$, $\mathrm{Cr}$, are very similar in shape. As the number of $d$-electrons increases from one in $\mathrm{Sc}$ to four in Cr, the Fermi level moves up the $d$-band in this series. As the DOS at the Fermi level increases, it enhances the probability of a magnetic instability. Indeed we find $\mathrm{CrH}_{2}$ to be antiferromagnetic with a magnetic moment of $1.5 \mu_{B}$ on the $\mathrm{Cr}$ atoms. The antiferromagnetic ordering is $51 \mathrm{meV} / \mathrm{f}$.u. more stable than the ferromagnetic ordering, which is $7 \mathrm{meV} / \mathrm{f}$.u. more stable than the non-polarized solution. In the other $\mathrm{TMH}_{2}$ we do not find magnetic effects.

Additional information on the type of bonding can be obtained from a Bader charge analysis. 61] In $\alpha-\mathrm{MgH}_{2}$ the Bader charges are $Q_{\mathrm{Mg}}=+1.59 e\left(\right.$ and $Q_{\mathrm{H}}=$ $-0.80 e$, since the compound is neutral), which confirms that this compound is dominantly ionic. The results for $\mathrm{TMH}_{2}$ are shown in Table 3.2. They indicate that the ionicity in $\mathrm{ScH}_{2}$ is comparable to that in $\mathrm{MgH}_{2}$. Furthermore, the ionicity decreases along the series $\mathrm{Sc}, \mathrm{Ti}, \mathrm{V}$ and $\mathrm{CrH}_{2}$. Comparison to Table 3.1 shows that the decrease in ionicity correlates with a decrease in formation enthalpy.

These results on the simple hydrides help us to analyze the electronic structure and bonding in $\mathrm{Mg}_{x} \mathrm{TM}_{(1-x)} \mathrm{H}_{2}$. We show results for the fluorite structure only, since that is the more stable structure over most of the composition range. As an example, Fig. 3.4 shows the calculated DOSs of $\mathrm{Mg}_{0.75} \mathrm{TM}_{0.25} \mathrm{H}_{2}$. One can qualitatively interpret these DOSs as a superposition of the DOSs of $\mathrm{MgH}_{2}$ and $\mathrm{TMH}_{2}$. The bonding states at low energy, comprising the first broad peak in the DOS, consist mainly of filled hydrogen states. The peaks close to the Fermi level are dominated by TM $d$-states. The Fermi level moves up the $d$-band through the series Sc, Ti, V, Cr. At higher energy we find the (unoccupied) Mg s states. The basic structure of the DOSs remains the same for all compositions $\mathrm{Mg}_{x} \mathrm{TM}_{(1-x)} \mathrm{H}_{2}$. As $x$ increases, the $\mathrm{TM} d$ 

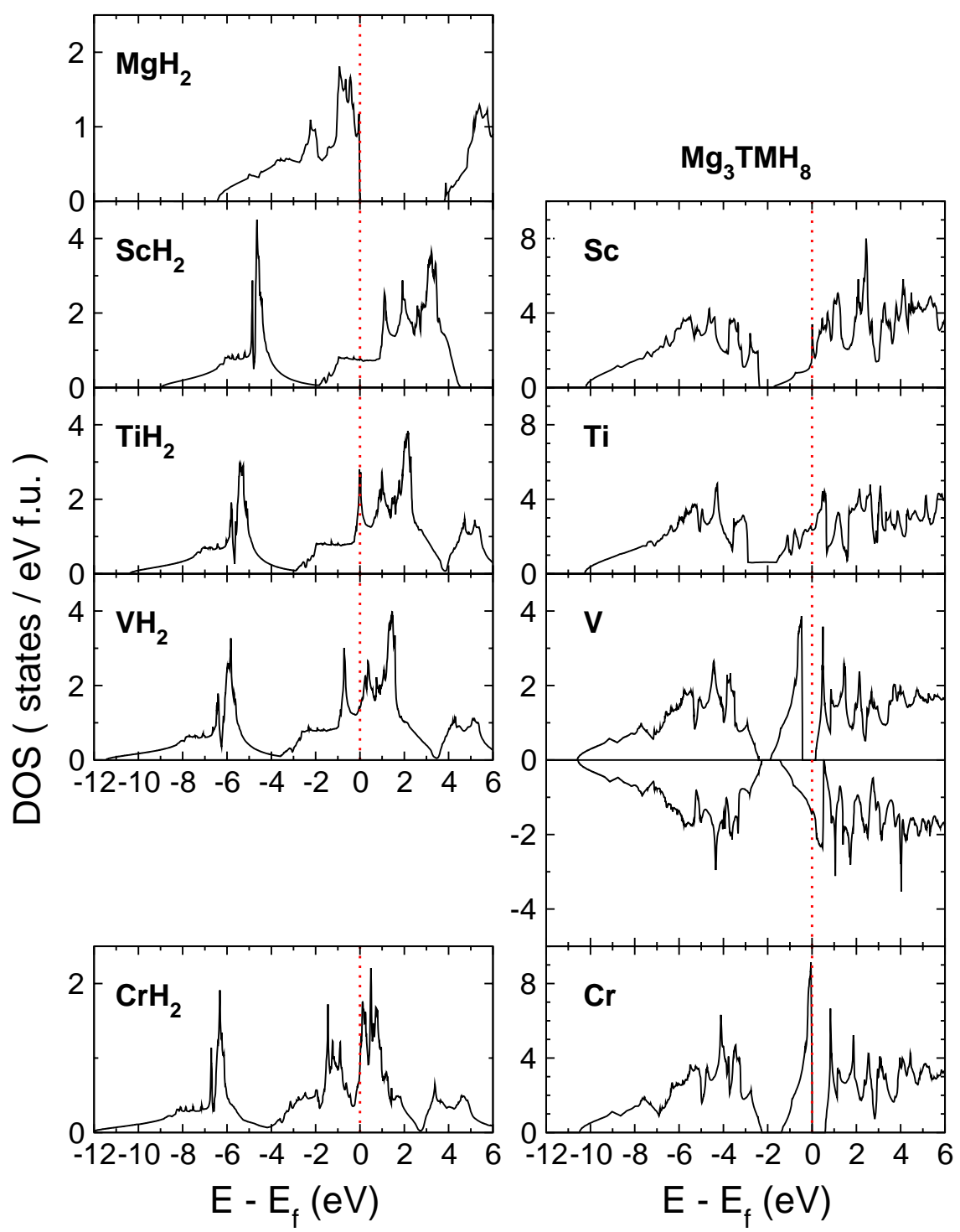

Figure 3.4: Densities of states of $\mathrm{MgH}_{2}$ and $\mathrm{TMH}_{2}$ (left column) and of $\mathrm{Mg}_{0.75} \mathrm{TM}_{0.25} \mathrm{H}_{2}$ (right column) for $\mathrm{TM}=\mathrm{Sc}, \mathrm{Ti}, \mathrm{V}, \mathrm{Cr}$. The shaded areas give the projected densities of states on the TM $d$ orbitals. For $\mathrm{CrH}_{2}$ the nonmagnetic DOS is given for simplicity reasons; $\mathrm{CrH}_{2}$ is antiferromagnetic (see text). 
contribution of course decreases. In addition, the TM $d$-peak becomes narrower with increasing $x$, as the distance between the TM atoms increases.

Narrowing of the $d$ peak can give rise to magnetic instabilities. The tendency to such instabilities increases along the series $\mathrm{Sc}, \mathrm{Ti}, \mathrm{V}$ and $\mathrm{Cr}$. The development of nonzero magnetic moments of course strongly depends upon the structure. Nevertheless, for $\mathrm{Mg}_{x} \mathrm{Sc}_{(1-x)} \mathrm{H}_{2}$ and $\mathrm{Mg}_{x} \mathrm{Ti}_{(1-x)} \mathrm{H}_{2}$ we see a tendency to form magnetic moments on the TMs only if $x \gtrsim 0.8$. For $\mathrm{Mg}_{x} \mathrm{~V}_{(1-x)} \mathrm{H}_{2}$ this occurs if $x \gtrsim 0.5$, and for $\mathrm{Mg}_{x} \mathrm{Cr}_{(1-x)} \mathrm{H}_{2}$ one can find magnetic instabilities over the whole composition range. Most of the structures have a finite DOS at the Fermi level, which, might indicate a metallic behavior. One cannot conclude this on the basis of a DOS alone, however, but should also critically evaluate possible localization and on-site correlation effects. There are a few exceptions. In particular cases low spin states can be more stable, such as for $\mathrm{Mg}_{0.75} \mathrm{Cr}_{0.25} \mathrm{H}_{2}$ in the fluorite structure. Cubic crystal field splitting by the hydrogens surrounding the $\mathrm{Cr}$ atom results in a gap between $e_{g}$ and $t_{2 g}$ states, the $e_{g}$ states being lowest in energy. The latter are filled by the four $d$ electrons of $\mathrm{Cr}$, which makes this particular structure insulating, see Fig. 3.4. The DOS of $\mathrm{Mg}_{0.75} \mathrm{~V}_{0.25} \mathrm{H}_{2}$ in the fluorite structure is explained by the same mechanism. However, as $\mathrm{V}$ only has three $d$ electrons, each $\mathrm{V}$ atom obtains a magnetic moment of $1 \mu_{B}$. The distance between the TM atoms is fairly large in most compositions that have nonzero magnetic moments, which suggests a small magnetic coupling between the TM atoms, a low Néel or Curie temperature, and paramagnetic behavior at room temperature. Exceptions are the Cr compounds with a substantial amount of $\mathrm{Cr}$, as discussed above.

A Bader charge analysis of $\mathrm{Mg}_{x} \mathrm{TM}_{(1-x)} \mathrm{H}_{2}$ can be made, similar to the simple hydrides. For all compositions $Q_{\mathrm{Mg}} \approx+1.6 e$, i.e. close to the value found in $\alpha-\mathrm{MgH}_{2}$. As an example the Bader charges on the $\mathrm{TM}$ and $\mathrm{H}$ atoms in $\mathrm{Mg}_{0.75} \mathrm{TM}_{0.25} \mathrm{H}_{2}$ are given in Table 3.2. The charge on the TM atoms decreases along the series $\mathrm{Sc}, \mathrm{Ti}$, $\mathrm{V}$, and $\mathrm{Cr}$ as in the simple hydrides, but compared to the latter, it is somewhat smaller on $\mathrm{V}$ and $\mathrm{Cr}$. The charges on the $\mathrm{H}$ atoms in $\mathrm{Mg}_{0.75} \mathrm{TM}_{0.25} \mathrm{H}_{2}$ are roughly the proportional average of the charges on the $\mathrm{H}$ atoms in $\mathrm{MgH}_{2}$ and $\mathrm{TMH}_{2}$. The charge analysis of the $\mathrm{Mg}_{x} \mathrm{TM}_{(1-x)} \mathrm{H}_{2}$ compounds is consistent with the bonding picture extracted from the DOSs.

\subsection{Discussion}

We discuss to what extend the Mg-TM alloys are suitable as hydrogen storage materials. The formation enthalpies of $\mathrm{Mg}_{x} \mathrm{TM}_{(1-x)} \mathrm{H}_{2}$ are shown in Fig. 4.6. 
Table 3.2: Bader charge analysis of $\mathrm{TMH}_{2}$ and $\mathrm{Mg}_{0.75} \mathrm{TM}_{0.25} \mathrm{H}_{2}$. All charges $Q$ are given in units of $e$.

\begin{tabular}{cccccc}
\hline \hline & \multicolumn{2}{c}{$\mathrm{TMH}_{2}$} & & \multicolumn{2}{c}{$\mathrm{Mg}_{0.75} \mathrm{TM}_{0.25} \mathrm{H}_{2}$} \\
\cline { 1 - 1 } $\mathrm{TM}$ & $Q_{\mathrm{TM}}$ & $Q_{\mathrm{H}}$ & & $Q_{\mathrm{TM}}$ & $Q_{\mathrm{H}}$ \\
$\mathrm{Sc}$ & +1.51 & -0.75 & & +1.57 & -0.80 \\
$\mathrm{Ti}$ & +1.17 & -0.59 & & +1.18 & -0.76 \\
$\mathrm{~V}$ & +1.09 & -0.55 & & +0.98 & -0.73 \\
$\mathrm{Cr}$ & +0.89 & -0.45 & & +0.68 & -0.69 \\
\hline \hline
\end{tabular}

Lightweight materials require a high content of magnesium, but to have a stable fluorite structure it should not exceed the critical composition $x_{c}$, as discussed in Sec. 3.4.1. We focus upon the composition $\mathrm{Mg}_{0.75} \mathrm{TM}_{0.25} \mathrm{H}_{2}$ in the following discussion. The calculated formation enthalpies are $-0.83,-0.59,-0.43$ and $-0.30 \mathrm{eV} /$ f.u. for $\mathrm{TM}=\mathrm{Sc}, \mathrm{Ti}, \mathrm{V}$, and $\mathrm{Cr}$, respectively. For applications the binding enthalpy of hydrogen in the lattice should be $\left.\lesssim 0.4 \mathrm{eV} / \mathrm{H}_{2}, 2-4\right]$ which indicates that the $\mathrm{Sc}$ and $\mathrm{Ti}$ compounds are too stable. The formation enthalpies of the $\mathrm{V}$ and $\mathrm{Cr}$ compounds could be in the right range. However, the parameter that is most relevant for hydrogen storage is the hydrogenation enthalpy. Assuming that the alloy does not dissociate upon dehydrogenation, the hydrogenation enthalpy corresponds the reaction

$$
\mathrm{Mg}_{x} \mathrm{TM}_{(1-x)}+\mathrm{H}_{2}(g) \longrightarrow \mathrm{Mg}_{x} \operatorname{TM}_{(1-x)} \mathrm{H}_{2} .
$$

To assess the hydrogenation enthalpy, one can break down the formation enthalpy associated with Eq. (3.1) into components, similar to the decomposition used in Ref. [37. We write the formation enthalpy as a sum of three terms. (i) The enthalpy required to make the Mg-TM alloy in the fcc structure from the elements in their most stable form. (ii) The energy required to expand the fcc lattice in order to incorporate the hydrogen atoms. (iii) The energy associated with inserting the hydrogen atoms. The results of this decomposition for $\mathrm{Mg}_{0.75} \mathrm{TM}_{0.25} \mathrm{H}_{2}$ are given in Fig. 3.5. To facilitate the discussion, a similar decomposition is shown for the simple hydrides, where (i) only consists of transforming the pure metal into the fcc structure. In contrast to Ref. [37, we use the spin-polarized fcc alloy for calculating the contributions (i) and (ii), as this will make the extraction of the hydrogenation enthalpy easier. In the cases where the magnetic moment is nonzero, we study both ferromagnetic and antiferromagnetic ordering. As for the simple hydrides, $\mathrm{Cr}$ compounds generally have an antiferromagnetic ordering. 
The lattice expansion energy (ii) of the compounds $\mathrm{Mg}_{0.75} \mathrm{TM}_{0.25} \mathrm{H}_{2}$ is $\leq 0.1 \mathrm{eV}$ for all TMs, see Fig. 3.5(b). It is in fact comparable to that of pure Mg, see Fig. 3.5(a). At the composition $\mathrm{Mg}_{0.75} \mathrm{TM}_{0.25} \mathrm{H}_{2}$, the effect on the energy of changing the unit cell volume is dominated by $\mathrm{Mg}$. For these compounds the lattice expansion only plays a minor role in the formation energy, in contrast to the simple hydrides, where the lattice expansion gives a significant contribution. The hydrogen insertion energies (iii) are also remarkably similar for the $\mathrm{Sc}$, Ti, and V compounds. Again this is in sharp contrast to the corresponding energies for the simple hydrides, which strongly depend on the TM. The hydrogen insertion energies for the compounds are in fact similar to that of pure Mg. At the composition $\mathrm{Mg}_{0.75} \mathrm{TM}_{0.25} \mathrm{H}_{2}$ also this energy is then dominated by $\mathrm{Mg}$. Only the compound $\mathrm{Mg}_{0.75} \mathrm{Cr}_{0.25} \mathrm{H}_{2}$ has a somewhat smaller hydrogen insertion energy. The reason for this is that the energy gained by magnetic ordering of the alloy $\mathrm{Mg}_{0.75} \mathrm{Cr}_{0.25}$ is relatively high, as compared to the other compounds. This contribution stabilizes the alloy with respect to the hydride, which is nonmagnetic.

The formation enthalpy of the fcc alloys $\mathrm{Mg}_{0.75} \mathrm{TM}_{0.25}$ (i) shows the largest variation as a function of the TM, relative to the contributions (ii) and (iii). Whereas the alloy formation energy is negative for $\mathrm{TM}=\mathrm{Sc}$, indicating that this alloy is stable, it is positive for $\mathrm{Ti}, \mathrm{V}$, and $\mathrm{Cr}$, meaning that these alloys are unstable. This result agrees with the experimental finding that of the Mg-TM alloys considered here, only a stable Mg-Sc alloy exists in bulk form. The substantial increase of the alloy formation enthalpy in the series $\mathrm{Sc}, \mathrm{Ti}, \mathrm{V}$, is largely responsible for the variation of the formation energy of the corresponding hydrides $\mathrm{Mg}_{0.75} \mathrm{TM}_{0.25} \mathrm{H}_{2}$. The alloy formation energy of $\mathrm{Mg}_{0.75} \mathrm{Cr}_{0.25}$ is similar to that of $\mathrm{Mg}_{0.75} \mathrm{~V}_{0.25}$, due to a relatively high spin-polarization energy, as discussed in the previous paragraph.

The hydrogenation enthalpy according to Eq. (3.3) can be determined by summing the contributions (ii) and (iii) of Fig. 3.5. Since the most stable structure of the alloys is not always the fcc structure, one should however subtract the energy required to convert the alloys from their most stable structure to an fcc structure. We find, for instance, that for $\mathrm{Mg}_{0.75} \mathrm{Ti}_{0.25}$ the fcc structure is $0.04 \mathrm{eV} /$ f.u. less stable than the hcp structure. Indeed thin film experiments on $\mathrm{Mg}_{x} \mathrm{Ti}_{(1-x)}$ yield an hcp structure. [19, 23 , For $\mathrm{Mg}_{0.75} \mathrm{Sc}_{0.25}$ the fcc structure is more stable than the hcp structure by 0.05 $\mathrm{eV} /$ f.u..

The calculated hydrogenation enthalpy of $\mathrm{Mg}_{0.75} \mathrm{Sc}_{0.25}$ is $-0.79 \mathrm{eV} /$ f.u., in good agreement with the experimental value of $-0.81 \mathrm{eV} /$ f.u..[19] The calculated hydrogenation enthalpy of $\mathrm{Mg}_{0.75} \mathrm{Ti}_{0.25}$ is $-0.76 \mathrm{eV} /$ f.u., which is in good agreement with the experimental value of $-0.81 \mathrm{eV} /$ f.u. of Ref. [26], obtained if the thin film cor- 

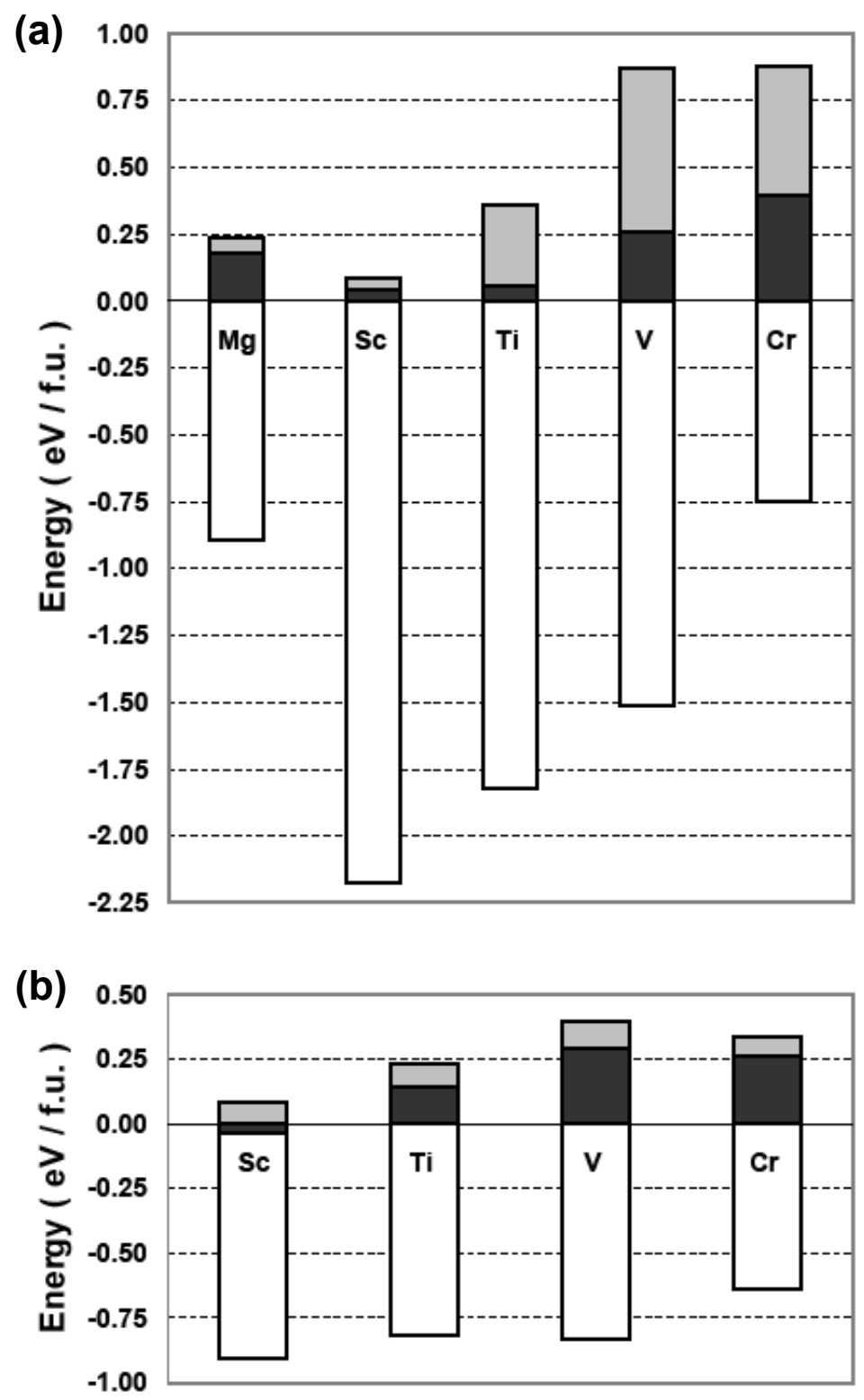

Figure 3.5: Decomposition of the formation energy into (i) the formation energy of the spin-polarized fcc metal $\mathrm{Mg}_{0.75} \mathrm{TM}_{0.25}$ (black), (ii) the lattice expansion energy (gray), and (iii) the hydrogen insertion energy (white); (a) the simple hydrides $\mathrm{MgH}_{2}, \mathrm{TMH}_{2}$; (b) $\mathrm{Mg}_{0.75} \mathrm{TM}_{0.25} \mathrm{H}_{2}$. 
rection suggested there is included. These hydrogenation enthalpies are remarkably similar to that of pure $\mathrm{Mg}$, strongly suggesting that alloying $\mathrm{Mg}$ with these $\mathrm{TMs}$ does not improve this energy as compared to pure $\mathrm{Mg}$. The most stable structures of $\mathrm{Mg}_{0.75} \mathrm{TM}_{0.25}, \mathrm{TM}=\mathrm{V}, \mathrm{Cr}$, are not known, but judging from the $\mathrm{Sc}$ and $\mathrm{Ti}$ compounds the energy difference between the fcc and the most stable structures will be small. Neglecting this energy difference upper bounds for the hydrogenation enthalpies of $\mathrm{Mg}_{0.75} \mathrm{~V}_{0.25}$ and $\mathrm{Mg}_{0.75} \mathrm{Cr}_{0.25}$ are -0.72 and $-0.57 \mathrm{eV} /$ f.u., respectively. Again this indicates that alloying Mg with these TMs does not improve the hydrogenation enthalpy substantially.

\subsection{Summary}

In summary, we have studied the structure and stability of $\mathrm{Mg}_{x} \mathrm{TM}_{(1-x)} \mathrm{H}_{2}, \mathrm{TM}$ $=\mathrm{Sc}, \mathrm{Ti}, \mathrm{V}, \mathrm{Cr}$, compounds by first-principles calculations. We find that for $x<$ $x_{c} \approx 0.8$ the fluorite structure is more stable than the rutile structure, whereas for $x>x_{c}$ the rutile structure is more stable. The density of states of these compounds is characterized by the valence bands being dominated by contributions from the hydrogen atoms, whereas the TMs have partially occupied $d$ states around the Fermi level. As $x$ increases and/or one moves down the TM series, the tendency to magnetic instabilities increases.

The formation enthalpy of $\mathrm{Mg}_{x} \mathrm{TM}_{1-x} \mathrm{H}_{2}$ can be tuned over a substantial range, i.e. $0-2 \mathrm{eV} /$ f.u., by varying TM and $x$. To a large part this reflects the variation of the formation enthalpy of the alloy $\mathrm{Mg}_{x} \mathrm{TM}_{(1-x)}$, however. Assuming that the alloys do not decompose upon dehydrogenation, the hydrogenation enthalpy then shows much less variation. For compounds with a high magnesium content $(x=0.75)$ it is close to that of pure Mg. Introducing a third metallic element may however be used to significantly improve the thermodynamics of (de)hydrogenation. The study of ternary alloys of $\mathrm{Mg}$ with transition metals and/or simple metals is actively pursued at present. 24, 62, 64]

The phase transition from the rutile to the fluorite structure in $\mathrm{Mg}_{x} \mathrm{TM}_{(1-x)} \mathrm{H}_{2}$, if $x$ falls below the critical composition $x_{c}$, correlates with the experimentally observed speeding up of the (de)hydrogenation kinetics in these compounds. A discussion of the kinetics is beyond the scope of the present paper. However, our preliminary calculations indicate that even in $\mathrm{MgH}_{2}$, hydrogen diffusion in the cubic (fluorite derived) $\beta-\mathrm{MgH}_{2}$ phase is much faster than in the rutile $\alpha-\mathrm{MgH}_{2}$ phase, both through a smaller formation energy for a hydrogen vacancy, as well as through a lower barrier for vacancy diffusion. 


\section{Acknowledgments}

This work is part of the research programs of "Advanced Chemical Technologies for Sustainability (ACTS)" and the "Stichting voor Fundamenteel Onderzoek der Materie (FOM)". The use of supercomputer facilities was sponsored by the "Stichting Nationale Computerfaciliteiten (NCF)". These institutions are financially supported by "Nederlandse Organisatie voor Wetenschappelijk Onderzoek (NWO)".

\section{Bibliography}

[1] See the special issue Toward a Hydrogen Economy, by R. Coontz and B. Hanson, Science 305, 957 (2004).

[2] A. Züttel, Materials Today 6, 24 (2003).

[3] A. Züttel, Naturwissenschaften 91, 157 (2004).

[4] L. Schlapbach and A. Züttel, Nature 414, 353 (2001).

[5] B. Bogdanovic, M. Felderhoff, S. Kaskel, A. Pommerin, K. Schlichte, and F. Schuth, Adv. Mater. 15, 1012 (2003).

[6] J. F. Stampfer, Jr., C. E. Holley, Jr., and J. F. Suttle, J. Am. Chem. Soc. 82, 3504 (1960).

[7] J. Huot, G. Liang, and R. Schulz, Appl. Phys. A: Mater. Sci. Process. 72, 187 (2001).

[8] W. Grochala and P. P. Edwards, Chem. Rev. 104, 1283 (2004).

[9] A. Zaluska, L. Zaluski, and J. O. Ström-Olsen, Appl. Phys. A: Mater. Sci. Process. 72, 157 (2001).

[10] M. Dornheim, N. Eigen, G. Barkhordarian, T. Klassen, and R. Bormann, Adv. Eng. Mater. 8 (2006).

[11] W. Li, C. Li, H. Ma, and J. Chen, J. Am. Chem. Soc. 129, 6710 (2007).

[12] R. W. P. Wagemans, J. H. van Lenthe, P. E. de Jongh, A. J. van Dillen, and K. P. de Jong, J. Am. Chem. Soc. 127, 16675 (2005).

[13] J. F. Pelletier, J. Huot, M. Sutton, R. Schulz, A. R. Sandy, L. B. Lurio, and S. G. J. Mochrie, Phys. Rev. B 63, 52103 (2001). 
[14] F. von Zeppelin, H. Reule, and M. Hirscher, J. Alloys Compd. 330, 723 (2002).

[15] X. Yao, C. Wu, A. Du, G. Q. Lu, H. Cheng, S. C. Smith, J. Zou, and Y. He, J. Phys. Chem. B 110, 11697 (2006).

[16] P. H. L. Notten, M. Ouwerkerk, H. van Hal, D. Beelen, W. Keur, J. Zhou, and H. Feil, J. Power Sources 129, 45 (2004).

[17] R. A. H. Niessen and P. H. L. Notten, Electrochem. Solid-State Lett. 8, A534 (2005).

[18] R. A. H. Niessen and P. H. L. Notten, J. Alloys Compd. 404, 457 (2005).

[19] W. P. Kalisvaart, R. A. H. Niessen, and P. H. L. Notten, J. Alloys Compd. 417, 280 (2006).

[20] R. A. H. Niessen, P. Vermeulen, and P. H. L. Notten, Electrochim. Acta 51, 2427 (2006).

[21] P. Vermeulen, R. A. H. Niessen, and P. H. L. Notten, Electrochem. Commun. 8, 27 (2006).

[22] D. M. Borsa, A. Baldi, M. Pasturel, H. Schreuders, B. Dam, R. Griessen, P. Vermeulen, and P. H. L. Notten, Appl. Phys. Lett. 88, 241910 (2006).

[23] D. Borsa, R. Gremaud, A. Baldi, H. Schreuders, J. Rector, B. Kooi, P. Vermeulen, P. Notten, B. Dam, and R. Griessen, Phys. Rev. B 75, 205408 (2007).

[24] P. Vermeulen, E. F. M. J. van Thiel, and P. H. L. Notten, Chem. Eur. J. 13, 9892 (2007).

[25] W. P. Kalisvaart, H. J. Wondergem, F. Bakker, and P. H. L. Notten, J. Mater. Res 22, 1640 (2007).

[26] R. Gremaud, C. P. Broedersz, D. M. Borsa, A. Borgschulte, P. Mauron, H. Schreuders, J. H. Rector, B. Dam, and R. Griessen, Adv. Mater. 19, 2813 (2007).

[27] K. H. J. Buschow, P. C. P. Bouten, and A. R. Miedema, Reports on Progress in Physics 45, 937 (1982).

[28] J. P. Perdew, K. Burke, and M. Ernzerhof, Phys. Rev. Lett. 77, 3865 (1996).

[29] P. E. Blöchl, Phys. Rev. B 50, 17953 (1994). 
[30] G. Kresse and D. Joubert, Phys. Rev. B 59, 1758 (1999).

[31] G. Kresse and J. Hafner, Phys. Rev. B 47, 558 (1993).

[32] G. Kresse and J. Furthmüller, Phys. Rev. B 54, 11169 (1996).

[33] M. Methfessel and A. T. Paxton, Phys. Rev. B 40, 3616 (1989).

[34] P. E. Blöchl, O. Jepsen, and O. K. Andersen, Phys. Rev. B 49, 16223 (1994).

[35] K. P. Huber and G. Herzberg, Molecular Spectra and Molecular Structure. IV. Constants of Diatomic Molecules (Van Nostrand Reinhold Co., 1979).

[36] J. D. Cox, D. D. Wagman, and V. A. Medvedev, CODATA Key Values for Thermodynamics (Hemisphere Publ., 1989).

[37] K. Miwa and A. Fukumoto, Phys. Rev. B 65, 155114 (2002).

[38] C. Wolverton, V. Ozolins, and M. Asta, Phys. Rev. B 69, 144109 (2004).

[39] L. Zhang, Y. Wang, T. Cui, Y. Li, Z. He, Y. Ma, and G. Zou, Phys. Rev. B 75, 144109 (2008).

[40] M. Bortz, B. Bertheville, G. Bottger, and K. Yvon, J. Alloys Compd. 287, L4 (1999).

[41] W. M. Mueller, J. P. Blackledge, and G. G. Libowitz, Metal Hydrides (Academic Press, New York, 1968).

[42] P. Villars, L. D. Calvert, and W. B. Pearson, Pearson's Handbook of Crystallographic Data for Intermetallic Phases (ASM International, Materials Park, OH, USA, 1991).

[43] C. A. Snavely and D. A. Vaughan, J. Am. Chem. Soc. 71, 313 (1949).

[44] M. Latroche, W. P. Kalisvaart, and P. H. L. Notten, J. Solid State Chem. 179, 3024 (2006).

[45] P. C. M. M. Magusin, W. P. Kalisvaart, P. H. L. Notten, and R. A. van Santen, Chem. Phys. Lett. 456, 55 (2008).

[46] P. Vermeulen, R. A. H. Niessen, D. M. Borsa, B. Dam, R. Griessen, and P. H. L. Notten, Electrochem. Solid-State Lett. 9, A520 (2006).

[47] G. Liang, J. Huot, S. Boily, A. V. Neste, and R. Schulz, J. Alloys Compd. 292, 247 (1999). 
[48] J. L. Bobet, C. Even, Y. Nakamura, E. Akiba, and B. Darriet, J. Alloys Compd. 298, 279 (2000).

[49] G. Liang and R. Schulz, J. Mater. Sci. 38, 1179 (2003).

[50] Y. J. Choi, J. Lu, H. Y. Sohn, and Z. Z. Fang, J. Power Sources 180, 491 (2008).

[51] D. Kyoi, T. Sato, E. Rönnebro, N. Kitamura, A. Ueda, M. Ito, S. Katsuyama, S. Hara, D. Noréus, and T. Sakai, J. Alloys Compd. 372, 213 (2004).

[52] D. Kyoi, E. Rönnebro, N. Kitamura, A. Ueda., M. Ito, S. Katsuyama, and T. Sakai, J. Alloys Compd. 361, 252 (2003).

[53] D. Kyoi, T. Sato, E. Rönnebro, Y. Tsuji, N. Kitamura, A. Ueda, M. Ito, S. Katsuyama, S. Hara, D. Noréus, et al., J. Alloys Compd. 375, 253 (2004).

[54] E. Rönnebro, D. Kyoi, H. Blomqvist, D. Noréus, and T. Sakai, J. Alloys Compd. 368, 279 (2004).

[55] E. Rönnebro, D. Kyoi, A. Kitano, Y. Kitano, and T. Sakai, J. Alloys Compd. 404, 68 (2005).

[56] A. V. Ruban, S. I. Simak, S. Shallcross, and H. L. .Skriver, Phys. Rev. B 67, 214302 (2003).

[57] S. Er, M. van Setten, G. de Wijs, and G. Brocks, in preparation (2009).

[58] P. Vajeeston, P. Ravindran, A. Kjekshus, and H. Fjellvåg, Phys. Rev. Lett. 89, 175506 (2002).

[59] J. Hafner, J. Phys. F: Met. Phys. 15, L43 (1985).

[60] M. J. van Setten, V. A. Popa, G. A. de Wijs, and G. Brocks, Phys. Rev. B 75, 35204 (2007).

[61] G. Henkelman, A. Arnaldsson, and H. Jónsson, Comput. Mater. Sci. 36, 354 (2006).

[62] M. J. van Setten, G. A. de Wijs, V. A. Popa, and G. Brocks, Phys. Rev. B 72, 073107 (2005).

[63] M. J. van Setten, G. A. de Wijs, and G. Brocks, Phys. Rev. B 76, 075125 (2007).

[64] C. P. Broedersz, R. Gremaud, B. Dam, R. Griessen, and O. M. Lovvik, Phys. Rev. B 77, 024204 (2008). 


\section{Chapter 4}

\section{First principles modelling of magnesium titanium hydrides}

\subsection{Abstract}

Mixing $\mathrm{Mg}$ with $\mathrm{Ti}$ leads to a hydride $\mathrm{Mg}_{x} \mathrm{Ti}_{(1-x)} \mathrm{H}_{2}$ with markedly improved (de)hydrogenation properties for $x \lesssim 0.8$, as compared to $\mathrm{MgH}_{2}$. Optically, thin films of $\mathrm{Mg}_{x} \mathrm{Ti}_{(1-x)} \mathrm{H}_{2}$ have a black appearance, which is remarkable for a hydride material. In this paper we study the structure and stability of $\mathrm{Mg}_{x} \mathrm{Ti}_{(1-x)} \mathrm{H}_{2}, x=0-1$ by firstprinciples calculations at the level of density functional theory. We give evidence for a fluorite to rutile phase transition at a critical composition $x_{\mathrm{c}}=0.8-0.9$, which correlates with the experimentally observed sharp decrease in (de)hydrogenation rates at this composition. The densities of states of $\mathrm{Mg}_{x} \mathrm{Ti}_{(1-x)} \mathrm{H}_{2}$ have a peak at the Fermi level, composed of Ti d states. Disorder in the positions of the Ti atoms easily destroys the metallic plasma, however, which suppresses the optical reflection. Interband transitions result in a featureless optical absorption over a large energy range, causing the black appearance of $\mathrm{Mg}_{x} \mathrm{Ti}_{(1-x)} \mathrm{H}_{2}$.

\subsection{Introduction}

Hydrogen promises to be a good candidate to replace carbon based fuels in the future 1]. The current inability to store hydrogen in a safe and sufficiently dense form obstructs its use in vehicles or in portable applications. A practical means of 
storage should yield a system that operates at moderate temperatures and releases hydrogen fast enough to feed a fuel cell [2. Storage should be reversible and the dehydrogenated system should adsorb hydrogen sufficiently fast. Typical target numbers for gravimetric and volumetric hydrogen densities in storage systems are 6 weight $\%$ (wt\%) and $0.045 \mathrm{~kg} / \mathrm{L}$.

The highest volumetric hydrogen densities among all possible storage forms are achieved in metal hydrides, where atomic hydrogen is bonded in the crystal lattice of bulk metals or alloys [3, 4]. Reasonable gravimetric hydrogen densities can be obtained if lightweight metals are used. For instance, the simple dihydride $\mathrm{MgH}_{2}$ has a gravimetric hydrogen density of $7.7 \mathrm{wt} \%$. However, $\mathrm{MgH}_{2}$ is so stable that releasing hydrogen at a pressure of 1 bar requires a temperature of around $300^{\circ} \mathrm{C}[5]$, which is impractically high for use in combination with PEM fuel cells. Moreover, the (de)hydrogenation kinetics of $\mathrm{MgH}_{2}$ is poor. Surface processes can hamper the kinetics, as the dissociation rate of hydrogen molecules on a $\mathrm{Mg}$ surface is small [6], and surface oxidation leads to the formation of a hydrogen diffusion barrier [7]. The surface kinetics can be improved by annealing [8] and by adding a catalyst [9]. Experimental studies also claim that the (de)hydrogenation kinetics of bulk $\mathrm{MgH}_{2}$ is very slow. It has been suggested that the kinetics is hampered by the rutile crystal structure of $\alpha-\mathrm{MgH}_{2}[10-12]$.

It is well known that $3 \mathrm{~d}$ transition metals (TMs) act as catalysts to improve the surface kinetics of hydrogen adsorption in $\mathrm{Mg}$ [13. To obtain this improvement usually only a few wt\% of TM is added to Mg. Recently it has been shown that also the bulk (de)hydrogenation kinetics can be improved markedly by adding TMs. One has to add a substantially larger amount, however, and make alloys $\mathrm{Mg}_{x} \mathrm{TM}_{(1-x)}$ with $\mathrm{TM}=\mathrm{Sc}$, Ti and $x \lesssim 0.8[10-12]$. The hydrides of these alloys have a cubic crystal structure quite unlike the $\alpha-\mathrm{MgH}_{2}$ rutile structure [14-17]. Obviously, to preserve a high gravimetric hydrogen density it is essential to use lightweight TMs. As Sc is too expensive to be used on a large scale, $\mathrm{Ti}$ is then the obvious choice. Thin $\mathrm{Mg}_{x} \operatorname{Ti}_{(1-x)}$ films, prepared by various experimental techniques, can be reversibly hydrogenated and dehydrogenated [12, 18 20]. The (de)hydrogenation kinetics is fast and increases gradually for alloys with increasing $\mathrm{Mg}$ content $x$, until a maximum at $x \approx 0.8$. If the $\mathrm{Mg}$ content is larger than this value, the kinetics becomes much slower.

Recent experimental studies reveal that $\mathrm{Mg}$-Ti-H thin films have interesting optical properties, which may lead to applications of these compounds other than hydrogen storage 19 21]. In the dehydrogenated state the films are highly reflective, whereas upon hydrogenation they become black, i.e. they have a low reflection and high absorption for light in the visible range. This would allow them to be used as switch- 
able smart coatings for solar panels, or as hydrogen sensors to detect the presence of hydrogen gas, for instance.

Despite the experimental interest in Mg-Ti hydrides, obtaining structural data has proven to be difficult as so far the bulk compounds have resisted synthesis under normal conditions. The only bulk Mg-Ti-H compound synthesized so far is $\mathrm{Mg}_{7} \mathrm{TiH}_{16}$, obtained by letting the dihydrides $\mathrm{MgH}_{2}$ and $\mathrm{TiH}_{2}$ react under extreme conditions of high pressure $(8 \mathrm{GPa})$ and temperature $(873 \mathrm{~K})[22,23]$. In contrast, thin films of $\mathrm{Mg}_{x} \mathrm{Ti}_{(1-x)} \mathrm{H}_{2}$ can be readily obtained for any composition $x$ by various deposition techniques. The limited amount of data on the structure, the thermodynamic stability and the electronic properties of $\mathrm{Mg}-\mathrm{Ti}-\mathrm{H}$ compounds has motivated the present first-principles computational study on $\mathrm{Mg}_{x} \mathrm{Ti}_{(1-x)} \mathrm{H}_{2}$.

First we consider the simple dihydrides $\mathrm{MgH}_{2}$ and $\mathrm{TiH}_{2}$ and subsequently characterize the properties of $\mathrm{Mg}_{x} \mathrm{Ti}_{(1-x)} \mathrm{H}_{2}$, focusing on the relative stability of possible cubic and rutile crystal structures. Limited first-principles calculations on ordered structures have indicated that a cubic fluorite-like crystal structure of $\mathrm{Mg}_{x} \mathrm{TM}_{(1-x)} \mathrm{H}_{2}$ is more stable than the rutile structure for a range of early TMs and $x \lesssim 0.8[24,25]$. Here we focus on $\mathrm{TM}=\mathrm{Ti}$ and study such structures in more detail, including the effects of disorder in the positions of the metal atoms, which is relevant for the experimental thin film studies. Our results suggest that the observed change in kinetics as a function of $\mathrm{Mg}$ content, is associated with a change in crystal structure. Finally, we calculate the optical properties of $\mathrm{Mg}_{x} \mathrm{Ti}_{(1-x)} \mathrm{H}_{2}$ and analyze them in terms of the compounds' structure and electronic structure. We show that the optical black state, which makes these compounds unique among the reversible hydrides so far, is an intrinsic property [26], unlike similar optical states of other Mg-TM-H thin films, which are caused by multiphase inhomogeneities in thin films [27, 28].

\subsection{Computational Details}

Most of the present calculations are performed at the level of the generalized gradient approximation (GGA) to density functional theory, using the projector augmented wave (PAW) technique and a plane wave basis set 29, 30, as implemented in the Vienna $A b$ initio Simulation Package (VASP) 31 33. Standard frozen core PAW potentials are used and the $\mathrm{H} 1 \mathrm{~s}, \mathrm{Mg} 2 \mathrm{~s}$, Ti $4 \mathrm{~s}$ and $3 \mathrm{~d}$ shells are treated as valence shells. As Ti has a partially filled $3 \mathrm{~d}$ shell we have considered the possibility of spin polarization and ferromagnetic or antiferromagnetic orderings. Unless otherwise mentioned explicitly, most of the compounds studied turn out to be paramagnetic, however. The main results are obtained using the Perdew Wang 91 (PW91) func- 
tional [34, but we have also performed some tests with the hybrid DFT/Hartree-Fock schemes B3LYP and HSE06 35 39].

We require that the total energy of each compound is converged to within 1 $\mathrm{meV} /$ atom with respect to the plane wave kinetic energy cutoff, which is assured by using a cutoff of $650 \mathrm{eV}$. Convergence with respect to the $k$-point sampling for the Brillouin zone (BZ) integration is tested independently on simple compounds using regular meshes of increasing density. We aim at converging total energies on a scale of $1 \mathrm{meV} /$ atom. This is obtained with the following $k$-point meshes for the simple unit cells: $12 \times 12 \times 8$ for hcp unit cells, $16 \times 16 \times 16$ for cubic, fcc or fluorite, unit cells and $12 \times 12 \times 16$ for bct or rutile unit cells. In studying alloys and their hydrides we construct supercells of these simple unit cells and keep the same $k$-point grid. Thus employing the equivalent k-points method one avoids relative $k$-point sampling errors and maintains the accuracy for all cell sizes 40 .

The molar volume of $\mathrm{Ti}\left(10.64 \mathrm{~cm}^{3}\right)$ is much smaller than that of $\mathrm{Mg}\left(14.00 \mathrm{~cm}^{3}\right)$, which makes finding the optimal cell parameters for $\mathrm{Mg}_{x} \mathrm{Ti}_{(1-x)} \mathrm{H}_{2}$ non-trivial. We start by keeping the atomic fractional coordinates and the $c / a$ ratio fixed and first carry out volume scans for each composition $x$ and crystal type individually. Values for the lattice parameters are then estimated from the total energy minima. These are then used as starting values for a full optimization of each composition and crystal type.

The cell parameters, including the cell volume, and the atomic positions within the cells are optimized by minimizing the forces and stresses with the conjugate gradient algorithm 41. During optimization Methfessel-Paxton smearing is used with a smearing parameter of $0.1 \mathrm{eV}$ [42. The criteria for self-consistency are set to $10^{-5}$ $\mathrm{eV}$ and $10^{-4} \mathrm{eV}$ for the total energy differences between two consecutive electronic and ionic steps, respectively. Structural relaxation is assumed to be complete if all the forces acting on atoms are smaller than $1 \mathrm{meV} / \AA$ in the simple cell studies, and $10 \mathrm{meV} / \AA$ in the supercell studies. After the structures are optimized, the total energies are recalculated self-consistently with the tetrahedron method [43. The latter technique is also used to calculate the electronic density of states (DOS).

The properties of the $\mathrm{H}_{2}$ molecule are calculated in a cubic cell with cell parameter $10 \AA$, using $\Gamma$-point sampling. The calculated $\mathrm{H}-\mathrm{H}$ bond length, binding energy, and vibrational frequency are $0.748 \AA,-4.56 \mathrm{eV}$ and $4351 \mathrm{~cm}^{-1}$, respectively, in good agreement with the experimental values of $0.741 \AA,-4.48 \mathrm{eV}$ and $4401 \mathrm{~cm}^{-1}$ [44].

To study the stability of $\mathrm{Mg}_{x} \mathrm{Ti}_{(1-x)} \mathrm{H}_{2}$ we look at the reaction

$$
x \mathrm{Mg}+(1-x) \mathrm{Ti}+\mathrm{H}_{2}(\text { gas }) \rightarrow \mathrm{Mg}_{x} \mathrm{Ti}_{(1-x)} \mathrm{H}_{2}
$$

In principle one should consider the change in Gibbs free energy $G(T, P)=U+$ 
$P V-T S$ of this reaction. As the thermodynamic properties of hydrogen gas are well documented [45, one can focus upon the solid phases involved in the reaction. In addition, the temperature dependence of $G$ of such solids tends to be relatively small over the temperature range of interest [46, 47]. A calculation of the change in enthalpy $H=U+P V$ at $T=0 \mathrm{~K}$ should therefore be adequate to assess the relative stability of metal hydrides. Since moreover the $P V$ contribution of solids can be neglected, it suffices to focus upon the change in energy $U$.

$U$ corresponds to the total energy of a system as obtained in a DFT calculation. The positions of the atomic nuclei are then fixed, which corresponds to $T=0 \mathrm{~K}$ for classical particles. However, hydrogen is such a light atom that quantum vibrational energies give contributions to the total energies that are not negligible, even at $T=0$ K. These zero-point energies (ZPEs) associated with atomic vibrations are obtained by solving the eigenvalue problem of the dynamical matrix [48. Slightly displacing an atom from its equilibrium position creates forces on the other atoms. The dynamical matrix can then be constructed by displacing each atom at a time and finite differencing the forces. We use symmetric displacements $d r= \pm 0.01 \AA$ for each atom and its three degrees of freedom. These calculations are carried out in supercells to properly account for the spatial range of the dynamical matrix.

The ZPE corrected reaction energies are then calculated as

$$
\Delta U^{\mathrm{ZPE}}=\sum_{p}\left(U_{p}+U_{p}^{\mathrm{vib}}\right)-\sum_{r}\left(U_{r}+U_{r}^{\mathrm{vib}}\right),
$$

where $r, p$ indicate the reactants and products of the reaction, respectively, $U$ is the total energy obtained from a DFT calculation and $U^{\mathrm{vib}}$ is the vibrational ZPE. As it turns out, the ZPE corrections result in a rather constant shift of the reaction energy for the range of compounds studied here. Therefore, we frequently give the reaction energy as $\Delta U$, calculated without $U_{p, r}^{\mathrm{vib}}$ contributions, and mention the ZPE corrections separately.

As we will discuss in Sec. 4.5.1, we employ two different strategies to model the structures. The first strategy consists of constructing a set of relatively simple ordered structures, inspired by the experimental structure of $\mathrm{Mg}_{7} \mathrm{TiH}_{16}$ [22, 23]. The structure of $\mathrm{Mg}_{x} \mathrm{Ti}_{(1-x)} \mathrm{H}_{y}$ as deposited in thin films is cubic with $\mathrm{Mg}$ and Ti atoms at fcc positions, but without a regular ordering of $\mathrm{Mg}$ and $\mathrm{Ti}$ atoms at these positions [15, 17. To model such disordered structures, our second strategy consists of employing special quasi-random structures (SQSs), which enable to model random alloys in a finite supercell [49. At each composition $\mathrm{Mg}_{x} \mathrm{Ti}_{(1-x)}$ of interest, the $\mathrm{Mg}$ and $\mathrm{Ti}$ atoms are distributed such, that their lower order correlation functions are equal to those of a perfect random alloy [50,51]. Hydrogen atoms are then inserted 
into similar positions as in the simple structures. We use a set of SQSs for each crystal structure (fluorite or rutile) and composition, with a total number of atoms ranging from 48 to 192. Again all cell parameters are optimized, as well as the positions of all atoms within the cell.

To model the optical response of $\mathrm{Mg}_{x} \mathrm{Ti}_{(1-x)} \mathrm{H}_{2}$ we calculate the dielectric function, consisting of interband and intraband contributions

$$
\varepsilon(\omega)=\varepsilon_{\text {inter }}(\omega)+\varepsilon_{\text {intra }}(\omega)
$$

which are calculated separately. The interband contribution $\varepsilon_{\text {inter }}$ is calculated in the independent particle approximation, using DFT eigenvalues and wave functions, i.e. neglecting exciton and local field effects. $\operatorname{Im}\left[\varepsilon_{\text {inter }}(\omega)\right]$ is calculated directly via the standard longitudinal expression, and $\operatorname{Re}\left[\varepsilon_{\text {inter }}(\omega)\right]$ is obtained by a KramersKronig transform [26, 52, 53]. As optical data on hydrides are usually obtained from micro- and nano-crystalline samples whose crystallites have a significant spread in orientation, we use the directionally averaged dielectric function.

Many metals and metal alloys undergo a metal-insulator transition upon full hydrogenation [27, 28, $53,58 . \mathrm{Mg}_{x} \mathrm{Ti}_{(1-x)} \mathrm{H}_{2}$ however retains a finite DOS at the Fermi level. Hence intraband transitions contribute to the dielectric function. We model $\varepsilon_{\text {intra }}(\omega)$ by a standard free electron plasma model, with the plasma frequency calculated from the $\mathbf{k}$-derivatives of the DFT eigenvalues [26, 59]. Once the full dielectric function $\varepsilon(\omega)$ is calculated, optical functions such as the extinction coefficient $\kappa(\omega)$ or the refractive index $n(\omega)$ are obtained via the standard expression

$$
\varepsilon(\omega)=[n(\omega)+i \kappa(\omega)]^{2}
$$

\subsection{Simple Dihydrides}

As discussed in the introduction, experimental information on the structure of $\mathrm{Mg}_{x} \mathrm{Ti}_{(1-x)} \mathrm{H}_{2}$ is limited, in particular concerning the positions of the hydrogen atoms. A sensible way to approach the problem is to assume that the structure is similar either to $\mathrm{MgH}_{2}$ or to $\mathrm{TiH}_{2}$. In this section we consider these simple dihydrides in more detail.

At standard temperature and pressure $\mathrm{MgH}_{2}$ has the rutile structure, space group $\mathrm{P}_{2} / \mathrm{mnm}$ (136), see figure 4.1. In this so-called $\alpha-\mathrm{MgH}_{2}$ phase, six hydrogen atoms surround each $\mathrm{Mg}$ atom in a distorted octahedron. The distance between $\mathrm{Mg}$ and two out of the six surrounding $\mathrm{H}$ atoms is $1.938 \AA$, and between $\mathrm{Mg}$ and the other four $\mathrm{H}$ 


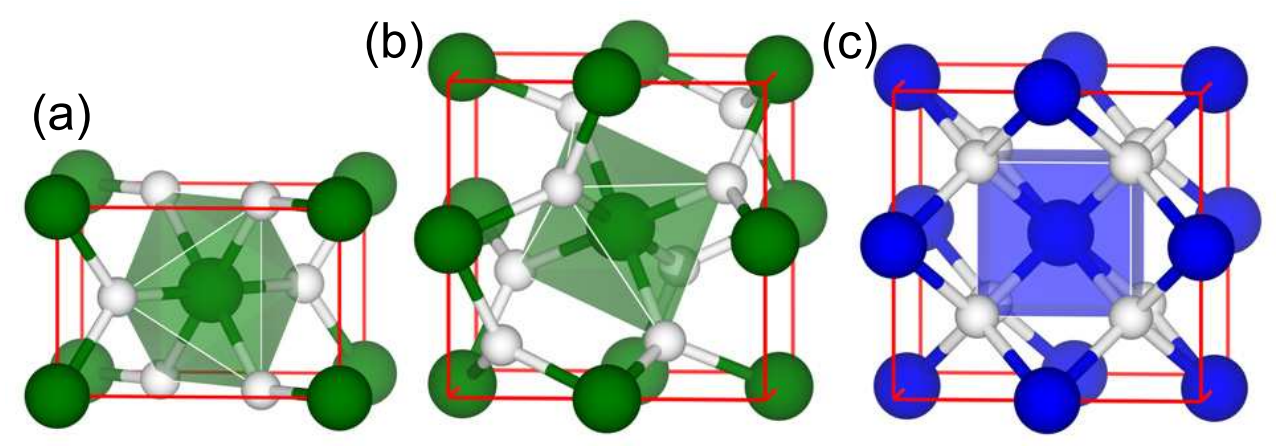

Figure 4.1: Crystal structures of $\alpha-\mathrm{MgH}_{2}$ (a), $\beta-\mathrm{MgH}_{2}$ (b), and $f-\mathrm{TiH}_{2}$ (c). $\mathrm{Mg}$, $\mathrm{Ti}$ and $\mathrm{H}$ atoms are shown as green, blue and white coloured spheres, respectively; the polyhedra indicate the coordination of the metal atoms by hydrogens. The images are made using the VESTA software 60.

atoms it is $1.951 \AA$. Each $\mathrm{H}$ atom is in the centre of a triangle with $\mathrm{Mg}$ atoms at the vertices. Experimentally it has been shown that an increase in the temperature and pressure to $923 \mathrm{~K}$ and $4 \mathrm{GPa}$, converts $\alpha-\mathrm{MgH}_{2}$ to a cubic form, called $\beta-\mathrm{MgH}_{2}$, with no further information about the positions of the $\mathrm{H}$ atoms 63. DFT calculations have suggested a structure for $\beta-\mathrm{MgH}_{2}$ 64]. Starting from a fluorite cubic structure with the hydrogen atoms on tetrahedral interstitial sites, space group Fm $\overline{3} \mathrm{~m}$ (225), structural optimization gives a cubic structure with reduced symmetry, space group $\mathrm{Pa} \overline{3}$ (205). This is accompanied by a decrease in the total energy of $0.24 \mathrm{eV} /$ f.u. and a volume expansion of $\sim 4 \%$. Each hydrogen atom is displaced from the centre of a tetrahedron to a base plane, where the coordination by $\mathrm{Mg}$ atoms is triangular with the three $\mathrm{Mg}$ atoms at a distance $d_{1}=1.911 \AA$, similar to the $\alpha$-phase. The fourth $\mathrm{Mg}$ atom of the original tetrahedron is at a distance $d_{2}=2.878 \AA$. Each $\mathrm{Mg}$ atom is octahedrally coordinated by six $\mathrm{H}$ atoms at a distance $d_{1}$. An additional two $\mathrm{H}$ atoms are at $d_{2}$. At zero pressure the optimized $\beta-\mathrm{MgH}_{2}$ structure is energetically unfavourable compared to the $\alpha-\mathrm{MgH}_{2}$ structure by $0.10 \mathrm{eV} /$ f.u. and its volume is $\sim 9 \%$ smaller. The optimized structural parameters obtained in our calculations are given in table 4.1. They are in good agreement with available experimental data.

The most stable phase of $\mathrm{TiH}_{2}$ at standard temperature and pressure has the fluorite structure, space group Fm $\overline{3} \mathrm{~m}$ (225), see figure 4.1, where the Ti atoms occupy the fcc lattice positions and hydrogen atoms reside at the tetrahedral interstitial positions. The calculated value of the $\mathrm{Ti}-\mathrm{H}$ bond length is $1.916 \AA$. We call this the $f-\mathrm{TiH}_{2}$ structure and to have a comparison between the two structural types of 
Table 4.1: Calculated and experimental structural parameters of simple metals and hydrides.

\begin{tabular}{|c|c|c|c|c|c|c|}
\hline Compound & $\begin{array}{l}\text { Space group } \\
\text { Unit cell }(\AA)\end{array}$ & & & $\mathrm{x}$ & $\mathrm{y}$ & $\mathrm{z}$ \\
\hline $\mathrm{Mg}$ & $\begin{array}{c}\mathrm{P} 6_{3} / \mathrm{mmc}(194) \\
a=3.190 ; c=5.180\end{array}$ & $\mathrm{Mg}$ & $2 \mathrm{c}$ & $1 / 3$ & $2 / 3$ & $1 / 4$ \\
\hline exp. 61 & $a=3.209 ; c=5.210$ & $\mathrm{Mg}$ & $2 \mathrm{c}$ & $1 / 3$ & $2 / 3$ & $1 / 4$ \\
\hline $\mathrm{Ti}$ & $\begin{array}{c}\mathrm{P} 6_{3} / \mathrm{mmc}(194) \\
a=2.916 ; c=4.631\end{array}$ & $\mathrm{Ti}$ & $2 \mathrm{c}$ & $1 / 3$ & $2 / 3$ & $1 / 4$ \\
\hline exp. 61 & $a=2.951 ; c=4.686$ & $\mathrm{Ti}$ & $2 \mathrm{c}$ & $1 / 3$ & $2 / 3$ & $1 / 4$ \\
\hline \multirow[t]{2}{*}{$\alpha-\mathrm{MgH}_{2}$} & $\mathrm{P} 4_{2} / \mathrm{mnm}(136)$ & $\mathrm{Mg}$ & $2 \mathrm{a}$ & 0 & 0 & 0 \\
\hline & $a=4.494 ; c=3.005$ & $\mathrm{H}$ & $4 \mathrm{f}$ & 0.3044 & 0.3044 & 0 \\
\hline \multirow[t]{2}{*}{$\exp .62$} & $a=4.501 ; c=3.010$ & $\mathrm{Mg}$ & $2 \mathrm{a}$ & 0 & 0 & 0 \\
\hline & & $\mathrm{H}$ & $4 f$ & 0.3044 & 0.3044 & 0 \\
\hline \multirow[t]{2}{*}{$\beta-\mathrm{MgH}_{2}$} & $\mathrm{~Pa} \overline{3}(205)$ & $\mathrm{Mg}$ & $4 a$ & 0 & 0 & 0 \\
\hline & $a=4.796$ & $\mathrm{H}$ & $8 c$ & 0.3464 & 0.3464 & 0.3464 \\
\hline \multirow[t]{2}{*}{$f-\mathrm{TiH}_{2}$} & $\mathrm{Fm} \overline{3} \mathrm{~m}(225)$ & $\mathrm{Ti}$ & $4 \mathrm{a}$ & 0 & 0 & 0 \\
\hline & $a=4.424$ & $\mathrm{H}$ & $8 c$ & $1 / 4$ & $1 / 4$ & $1 / 4$ \\
\hline \multirow[t]{2}{*}{ exp. 61 } & $a=4.454$ & $\mathrm{Ti}$ & $4 a$ & 0 & 0 & 0 \\
\hline & & $\mathrm{H}$ & $8 \mathrm{c}$ & $1 / 4$ & $1 / 4$ & $1 / 4$ \\
\hline \multirow[t]{2}{*}{$r-\mathrm{TiH}_{2}$} & $\mathrm{P} 4_{2} / \mathrm{mnm}(136)$ & $\mathrm{Ti}$ & $2 \mathrm{a}$ & 0 & 0 & 0 \\
\hline & $a=3.991 ; c=3.080$ & $\mathrm{H}$ & $4 \mathrm{f}$ & 0.322 & 0.322 & 0 \\
\hline
\end{tabular}

dihydrides we also construct a rutile form of $\mathrm{TiH}_{2}$, and call it $r-\mathrm{TiH}_{2}$ in table 4.1 . This artificial rutile structure does not exist in nature to our knowledge. It has a $13 \%$ larger volume than $f-\mathrm{TiH}_{2}$, and its total energy is $0.65 \mathrm{eV} /$ f.u. higher.

The formation energies of the simple hydrides are defined as the reaction energies corresponding to (4.1) for $x=0,1$. The calculated values with and without vibrational ZPE corrections are given in table 4.2. ZPEs tend to destabilize the metal hydrides. By far the biggest contribution stems from the difference between the vibrational frequencies of the hydrogen atoms in the metal hydride and in the gas phase $\mathrm{H}_{2}$ molecules. The ZPEs of the metals are small, i.e. 0.031 and $0.033 \mathrm{eV} /$ atom for $\mathrm{Mg}$ and $\mathrm{Ti}$, respectively (calculated with a $3 \times 3 \times 2$ hcp supercell). In general, the more compact the metal hydride structure, the less space for the hydrogen atoms to vibrate, and the higher their vibrational frequencies and the ZPE are. The volumes per formula unit are $30.34 \AA^{3}$ for $\alpha-\mathrm{MgH}_{2}$ and $21.64 \AA^{3}$ for $f-\mathrm{TiH}_{2}$. The calculated 
Table 4.2: Calculated formation energies in $\mathrm{eV}$ without $(\Delta U)$ and with $\left(\Delta U^{\mathrm{ZPE}}\right)$ vibrational zero point energy corrections, cf. (4.2), compared to experimental values $\left(\Delta U^{\exp }\right)$. The latter are taken from 65] unless indicated otherwise.

\begin{tabular}{lccc}
\hline \hline Compound & $\Delta U$ & $\Delta U^{\mathrm{ZPE}}$ & $\Delta U^{\exp }$ \\
\hline$\alpha-\mathrm{MgH}_{2}$ & -0.657 & -0.558 & $-0.682-0.694-0.771$ \\
& & & $-0.772[66]-0.779[67]$ \\
$\alpha-\mathrm{TiH}_{2}$ & -1.467 & -1.279 & $-1.297-1.351-1.378$ \\
& & & $-1.450[68]-1.496$ \\
\hline \hline
\end{tabular}

Table 4.3: Calculated formation energies of $\alpha-\mathrm{MgH}_{2}$ in $\mathrm{eV}$, obtained with different PAW pseudopotentials for $\mathrm{Mg}$ and different DFT functionals or hybrid DFT/Hartree-Fock schemes. The ZPE correction has been calculated at the PW91 level only.

\begin{tabular}{llcc}
\hline \hline Mg valence & functional & $\Delta U$ & $\Delta U^{\mathrm{ZPE}}$ \\
\hline $3 \mathrm{~s}$ & PW91 & -0.657 & -0.558 \\
$2 \mathrm{p} 3 \mathrm{~s}$ & PW91 & -0.648 & -0.549 \\
$3 \mathrm{~s}$ & HSE06 & -0.25 & -0.15 \\
$3 \mathrm{~s}$ & B3LYP & -0.95 & -0.85 \\
\hline \hline
\end{tabular}

ZPEs are $0.406 \mathrm{eV} /$ f.u. and $0.501 \mathrm{eV} /$ f.u., respectively. These are calculated using a $2 \times 2 \times 3$ rutile supercell for $\mathrm{MgH}_{2}$, containing 72 atoms, and a $2 \times 2 \times 2$ fluorite supercell for $\mathrm{TiH}_{2}$, containing 96 atoms. The calculated $\mathrm{ZPE}$ of a $\mathrm{H}_{2}$ molecule is $0.276 \mathrm{eV}$. Consequently, including ZPE corrections destabilizes $\alpha-\mathrm{MgH}_{2}$ by approximately 0.1 $\mathrm{eV}$, whereas the more compact $f-\mathrm{TiH}_{2}$ is destabilized by almost $0.2 \mathrm{eV}$, see table 4.2 .

The experimental numbers obtained for the formation energy of $\mathrm{TiH}_{2}$ range from -1.30 to $-1.50 \mathrm{eV}$. The calculated numbers are in good agreement with these results. Experimental values of the formation energy of $\mathrm{MgH}_{2}$ range from -0.68 to -0.78 $\mathrm{eV}$. The calculated values overestimate these numbers somewhat, in particular if one includes ZPEs. Such differences between calculated and experimental formation energies are also found in other simple alkali and alkaline earth hydrides and seem to be typical for the use of GGA functionals such as PW91 or PBE. To see whether the calculated values can be improved, we test the use of a hard PAW pseudopotential for $\mathrm{Mg}$, which includes the $2 \mathrm{p}$ shell as valence electrons, as well as the use of the hybrid DFT/Hartree-Fock schemes B3LYP and HSE06. The results, listed in table 4.3, show 

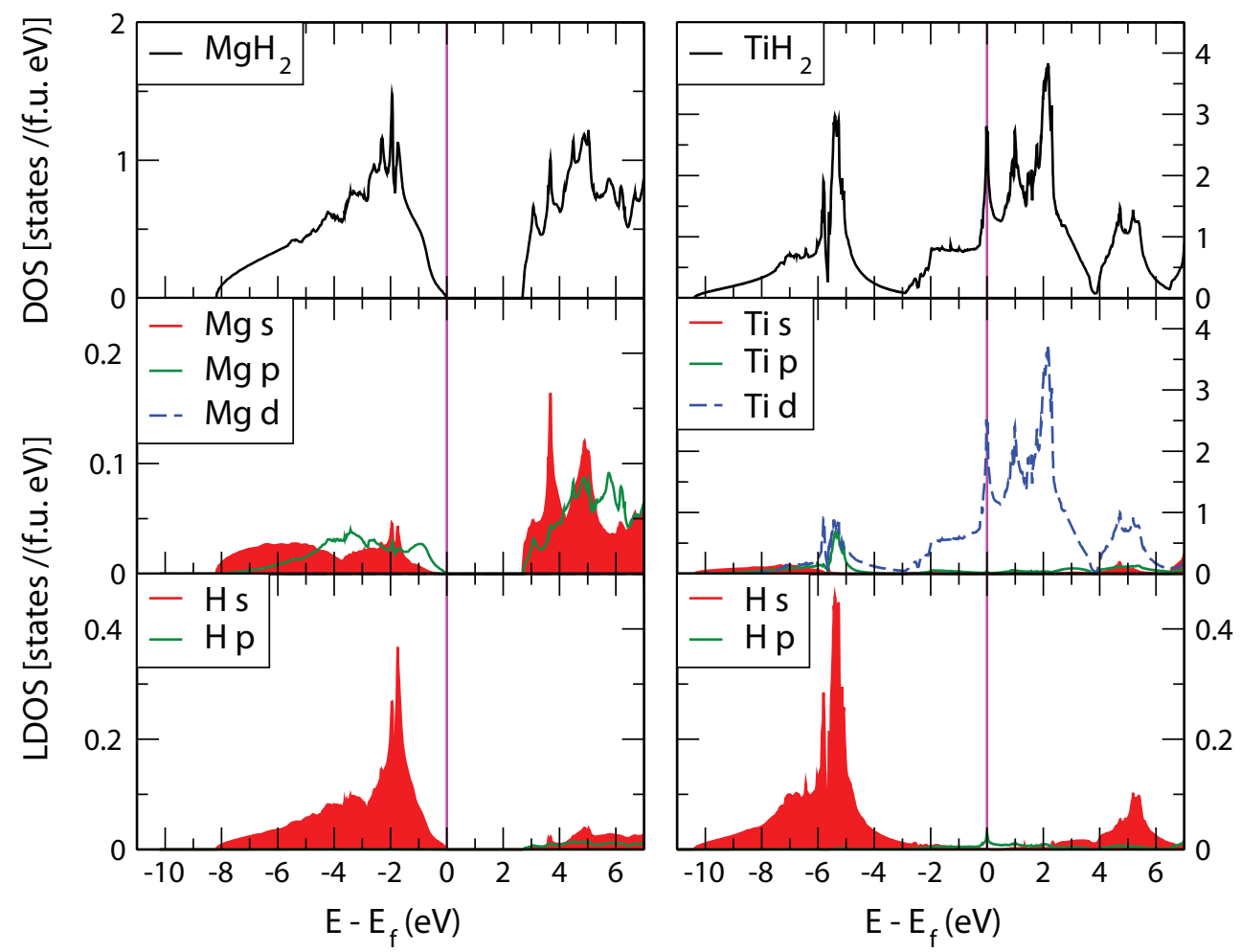

Figure 4.2: Electronic density of states (DOS) of $\beta-\mathrm{MgH}_{2}$ and $f-\mathrm{TiH}_{2}$. The origin of the energy scale is set at the top of the valence band. The top figure gives the total DOS, and the middle and bottom figures represent the local DOS, projected on the atoms; s, p and d contributions are shown in red, green and blue, respectively. The regions under the s curves are shaded and the $\mathrm{d}$ curves are dashed.

that the effect of using a hard PAW potential is minor. Hybrid DFT/Hartree-Fock schemes do not necessarily give an improvement over DFT/GGA functionals. The HSE06 and B3LYP schemes lead to formation energies of $\mathrm{MgH}_{2}$ that are respectively much larger and somewhat smaller than the experimental one. Since in the following we are foremost interested in energy differences between crystal structures, we stick to using the PW91 GGA functional.

$\alpha-\mathrm{MgH}_{2}$ is an insulator with an experimental optical band gap of $5.6 \mathrm{eV}$ [69. As usual DFT calculations underestimate this gap [53]; the value obtained for $\alpha-\mathrm{MgH}_{2}$ is $3.8 \mathrm{eV}$, whereas the calculated gap for $\beta-\mathrm{MgH}_{2}$ is $2.7 \mathrm{eV}$. The electronic (projected) densities of states ((P)DOS) of the cubic simple dihydrides $\beta-\mathrm{MgH}_{2}$ and $f-\mathrm{TiH}_{2}$ are 
shown in figure 4.2. The fact that many fully hydrided simple metals and alloys are insulators can be understood from simple chemical arguments [53, 55 58. The $\mathrm{Mg}$ atoms almost fully donate their valence $(2 \mathrm{~s})$ electrons to the $\mathrm{H}$ atoms. Indeed the PDOS in figure 4.2 shows that the valence bands have a predominant $\mathrm{H}$ 1s character, whereas the $\mathrm{Mg} 2 \mathrm{~s}$ states emerge in the conduction bands. In contrast, $\mathrm{TiH}_{2}$ is metallic with a considerable DOS at the Fermi level. The PDOS shows that the states around the Fermi energy have a dominant $\mathrm{Ti} 3 \mathrm{~d}$ character, whereas, as for $\mathrm{MgH}_{2}$, the lower valence bands are dominantly $\mathrm{H}$ 1s. It suggests that in $\mathrm{TiH}_{2}$ two electrons per metal atom are transferred to hydrogen and, as $\mathrm{Ti}$ atoms have four valence electrons $\left(\mathrm{s}^{2} \mathrm{~d}^{2}\right)$, they remain in an open-shell configuration in the hydride. Figure 4.2 illustrates that the Ti d electrons participate significantly less than the $\mathrm{s}$ electrons to the bonding to $\mathrm{H}$ atoms.

\section{5 $\operatorname{Mg}_{x} \operatorname{Ti}_{(1-x)} \mathrm{H}_{2}$}

\subsubsection{Structures}

The only detailed structure of $\mathrm{Mg}_{x} \mathrm{Ti}_{(1-x)} \mathrm{H}_{2}$ available from experiment is for the composition $x=0.875$. In the $\mathrm{Mg}_{7} \mathrm{TiH}_{16}$ high pressure phase the metal atoms are in fcc positions and are ordered as in the $\mathrm{Ca}_{7} \mathrm{Ge}$ structure [22, 23]. The $\mathrm{H}$ atoms are at interstitial sites, but displaced from their ideal tetrahedral positions. This structure has 96 atoms in the unit cell. We use it as a starting point to construct relatively simple, fluorite-type structures for other compositions $x$. For $x=0.125,0.875$ we use the $\mathrm{Ca}_{7} \mathrm{Ge}$ structure to order the metal atoms, for $x=0.25,0.75$ the $\mathrm{Cu}_{3} \mathrm{Au}\left(L 1_{2}\right)$ structure, and for $x=0.5$ the $\mathrm{CuAu}\left(L 1_{0}\right)$ structure. The $\mathrm{H}$ atoms are placed at or close to tetrahedral interstitial positions. In each of the structures and compositions the cell parameters are optimized, as well as the positions of all atoms within the cell. Care is taken to allow for breaking the symmetry in the atomic positions, as the hydrogen atoms are often displaced from their ideal tetrahedral positions. Although strictly speaking such structures are then no longer ideal fluorite structures anymore, we still use the term fluorite in the following.

The optimized fluorite structures are given in table 4.4. Most of these structures are cubic, except $\mathrm{Mg}_{0.5} \mathrm{Ti}_{0.5} \mathrm{H}_{2}$, which is tetragonally distorted. Comparing the calculated and the experimental structures of $\mathrm{Mg}_{0.875} \mathrm{Ti}_{0.125} \mathrm{H}_{2}$ one observes that the calculated lattice parameter is $2 \%$ smaller than the experimental one. Although such a result is not uncommon for DFT calculations, note that the differences between calculated and experimental lattice parameters of $\mathrm{MgH}_{2}$ and $\mathrm{TiH}_{2}$ are much smaller, 
Table 4.4: Optimized cell parameters and atomic positions of the fluoritetype simple $\mathrm{Mg}_{x} \mathrm{Ti}_{(1-x)} \mathrm{H}_{2}$ structures.

\begin{tabular}{|c|c|c|c|c|c|}
\hline \multicolumn{6}{|l|}{ Compound, Space group } \\
\hline $\mathrm{Mg}_{0.125} \mathrm{Ti}_{0.875} \mathrm{H}_{2}$ & $\mathrm{Mg}$ & $4 a$ & 0 & 0 & 0 \\
\hline Fm̄̄m (225) & Ti1 & $4 b$ & $1 / 2$ & $1 / 2$ & $1 / 2$ \\
\hline \multirow[t]{3}{*}{$a=8.8896$} & Ti2 & $24 d$ & 0 & $1 / 4$ & $1 / 4$ \\
\hline & $\mathrm{H} 1$ & $32 f$ & 0.1285 & 0.1285 & 0.1285 \\
\hline & $\mathrm{H} 2$ & $32 f$ & 0.3755 & 0.3755 & 0.3755 \\
\hline $\mathrm{Mg}_{0.25} \mathrm{Ti}_{0.75} \mathrm{H}_{2}$ & $\mathrm{Ti}$ & $3 c$ & 0 & $1 / 2$ & $1 / 2$ \\
\hline Pm̄̄m (221) & $\mathrm{Mg}$ & $1 a$ & 0 & 0 & 0 \\
\hline$a=4.4648$ & $\mathrm{H}$ & $8 g$ & 0.2566 & 0.2566 & 0.2566 \\
\hline $\mathrm{Mg}_{0.5} \mathrm{Ti}_{0.5} \mathrm{H}_{2}$ & Ti1 & $1 a$ & 0 & 0 & 0 \\
\hline $\mathrm{P} 4 / \mathrm{mmm}(123)$ & Ti2 & $1 c$ & $1 / 2$ & $1 / 2$ & 0 \\
\hline$a=4.4720$ & $\mathrm{Mg}$ & $2 e$ & 0 & $1 / 2$ & $1 / 2$ \\
\hline$c=4.6544$ & $\mathrm{H}$ & $8 r$ & 0.25 & 0.25 & 0.2352 \\
\hline $\mathrm{Mg}_{0.75} \mathrm{Ti}_{0.25} \mathrm{H}_{2}$ & $\mathrm{Ti}$ & $1 a$ & 0 & 0 & 0 \\
\hline Pm̄̄m (221) & $\mathrm{Mg}$ & $3 c$ & 0 & $1 / 2$ & $1 / 2$ \\
\hline$a=4.6208$ & $\mathrm{H}$ & $8 g$ & 0.2432 & 0.2432 & 0.2432 \\
\hline $\mathrm{Mg}_{0.875} \mathrm{Ti}_{0.125} \mathrm{H}_{2}$ & $\mathrm{Ti}$ & $4 a$ & 0 & 0 & 0 \\
\hline Fm $\overline{3} \mathrm{~m}(225)$ & Mg1 & $4 b$ & $1 / 2$ & $1 / 2$ & $1 / 2$ \\
\hline \multirow[t]{3}{*}{$a=9.3565$} & Mg2 & $24 d$ & 0 & $1 / 4$ & $1 / 4$ \\
\hline & H1 & $32 f$ & 0.1194 & 0.1194 & 0.1194 \\
\hline & $\mathrm{H} 2$ & $32 f$ & 0.3725 & 0.3725 & 0.3725 \\
\hline $\mathrm{Mg}_{0.875} \mathrm{Ti}_{0.125} \mathrm{H}_{2} \exp .23$ & $\mathrm{H} 1$ & $32 f$ & $0.094(2)$ & $0.094(2)$ & $0.094(2)$ \\
\hline$a=9.564(2)$ & $\mathrm{H} 2$ & $32 f$ & $0.365(2)$ & $0.365(2)$ & $0.365(2)$ \\
\hline
\end{tabular}

see table 4.1. The Wyckoff positions and the hydrogen-metal coordinations of the calculated and experimental structures of $\mathrm{Mg}_{0.875} \mathrm{Ti}_{0.125} \mathrm{H}_{2}$ agree, but the absolute difference between the calculated and experimental $\mathrm{H}$ positions is $0.1-0.4 \AA$. This is not a volume effect, since optimizing the structure using the experimental or the calculated lattice parameter gives the same $\mathrm{H}$ positions. We have also checked that breaking the symmetry by random displacement of the $\mathrm{H}$ atoms yields the same optimized Fm $\overline{3} \mathrm{~m}$ (225) structure. From our calculations the experimental structure is $0.766 \mathrm{eV} /$ f.u. higher in energy. 


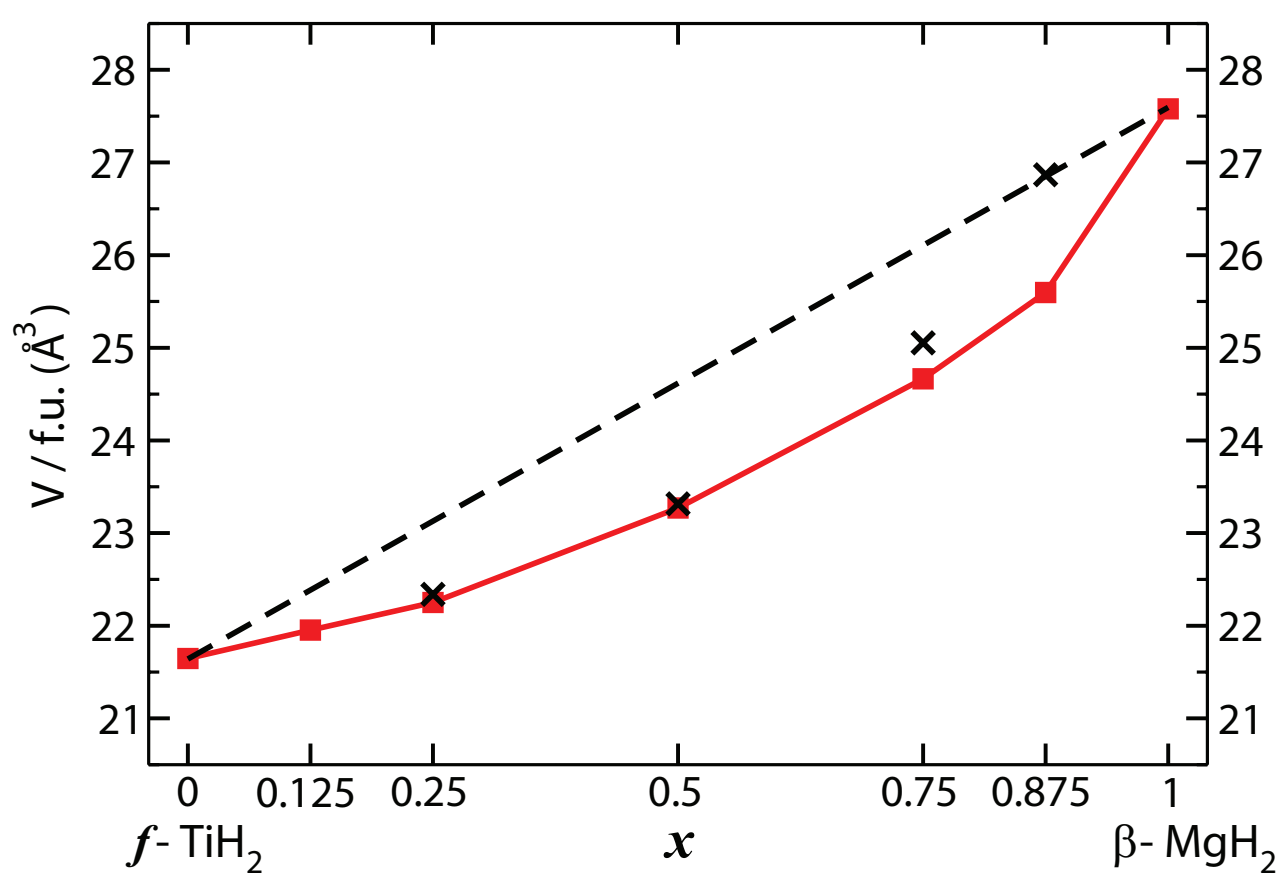

Figure 4.3: The volumes per formula unit (V/f.u) in $\AA^{3}$ of $\mathrm{Mg}_{x} \mathrm{Ti}_{(1-x)} \mathrm{H}_{2}$ in the ordered fluorite structures, as a function of the composition $x$. The dashed line marks a linear interpolation between $f-\mathrm{TiH}_{2}$ and $\beta-\mathrm{MgH}_{2}$. The crosses mark the volumes of the special quasi-random structures.

The calculated tetrahedral environment of a $\mathrm{H} 1$ atom in $\mathrm{Mg}_{0.875} \mathrm{Ti}_{0.125} \mathrm{H}_{2}$ consists of a Ti atom at a distance of $1.935 \AA$ and three Mg2 atoms at $2.058 \AA$; the experimental distances are $1.557 \AA$ and $2.293 \AA$, respectively. The calculated tetrahedral environment of a $\mathrm{H} 2$ atom consists of a Mg1 atom at $2.066 \AA$ and three $\mathrm{Mg} 2$ atoms at $2.013 \AA$ compared to experimental distances of $2.236 \AA$ and $2.021 \AA$. The calculated $\mathrm{H}$ - Ti distance is close to the $1.916 \AA$ found in $\mathrm{TiH}_{2}$, see table 4.1, whereas the experimental distance is significantly smaller. In fact, for all compositions in table 4.4 we find $\mathrm{H}-\mathrm{Ti}$ distances in the range 1.91-1.94 $\AA$. From the calculated $\mathrm{H}-\mathrm{Mg}$ distances one observes that the $\mathrm{H}$ atoms are closer to ideal tetrahedral positions than in the experimental structure. From the positions of the $\mathrm{H} 2$ atoms it is clear, however, that both the calculated and the experimental structure are closer to an ideal fluorite structure than to $\beta-\mathrm{MgH}_{2}$.

One should note that the experimental structure of $\mathrm{Mg}_{7} \mathrm{TiH}_{16}$ is based upon room temperature XRD data. As XRD measures the electronic charge distribution, mod- 

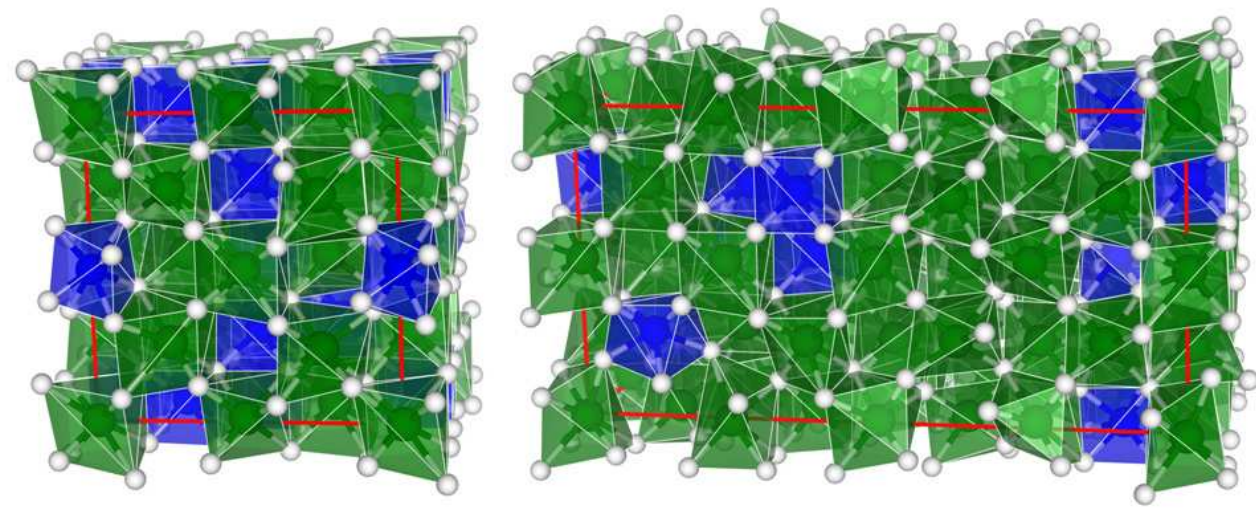

Figure 4.4: Cells to model special quasi-random structures of $\mathrm{Mg}_{x} \mathrm{Ti}_{(1-x)} \mathrm{H}_{2}$ for $x=0.75$ (left image) and $x=0.875$ (right image) compositions. $\mathrm{Mg}, \mathrm{Ti}$ and $\mathrm{H}$ atoms are shown as green, blue and white coloured spheres, respectively; the polyhedra indicate the coordination of the metal atoms by hydrogens 60 .

elling the data with atomic scattering functions introduces errors on the positions of light atoms such as hydrogen. In particular, the asymmetry in the hydrogen positions is sometimes exaggerated. In that case low temperature neutron diffraction can give a structure that is much closer to the calculated structure [46, 70].

The calculated volumes of the fluorite structures of $\mathrm{Mg}_{x} \mathrm{Ti}_{(1-x)} \mathrm{H}_{2}$ as a function of the composition $x$, normalized per formula unit, are shown in figure 4.3. According to Zen's law of additive volumes one would expect a linear dependence [71]. The curves deviate slightly, but distinctly, from straight lines, with a maximum deviation of $5.4 \%$ at $x=0.5$. This deviation is consistent with the experimental observations on $\mathrm{Mg}_{x} \operatorname{Ti}_{(1-x)} \mathrm{H}_{2}$ [12, 15]. It is also observed in simple metal alloys [71.

The binary phase diagram of $\mathrm{Mg}$ and $\mathrm{Ti}$ indicates that they do not form a stable bulk alloy. It is however claimed that synthesis by mechanical alloying may be possible 72. In addition, thin films of $\operatorname{Mg}_{x} \mathrm{Ti}_{(1-x)}$ are readily made, which can be reversibly hydrogenated [10, 12, 15, 18, 20. The crystal structure of $\mathrm{Mg}_{x} \mathrm{Ti}_{(1-x)} \mathrm{H}_{y}$ in thin films, $x \lesssim 0.8, y \approx 1-2$, is cubic, with the $\mathrm{Mg}$ and Ti atoms at fcc positions, but no detectable regular ordering of $\mathrm{Mg}$ and $\mathrm{Ti}$ atoms at these positions [15, 17. The positions of the $\mathrm{H}$ atoms have not been determined from experiment.

To model such disordered structures, we perform calculations on SQSs, which enable to model random alloys in a finite supercell. We use a 32 atom supercell to model SQSs of fcc $\mathrm{Mg}_{x} \mathrm{Ti}_{1-x}$ for $x=0.25,0.5$, and 0.75 , and a 64 atom supercell for 0.875 [50. Inserting hydrogen atoms in tetrahedral interstitial positions then gives 


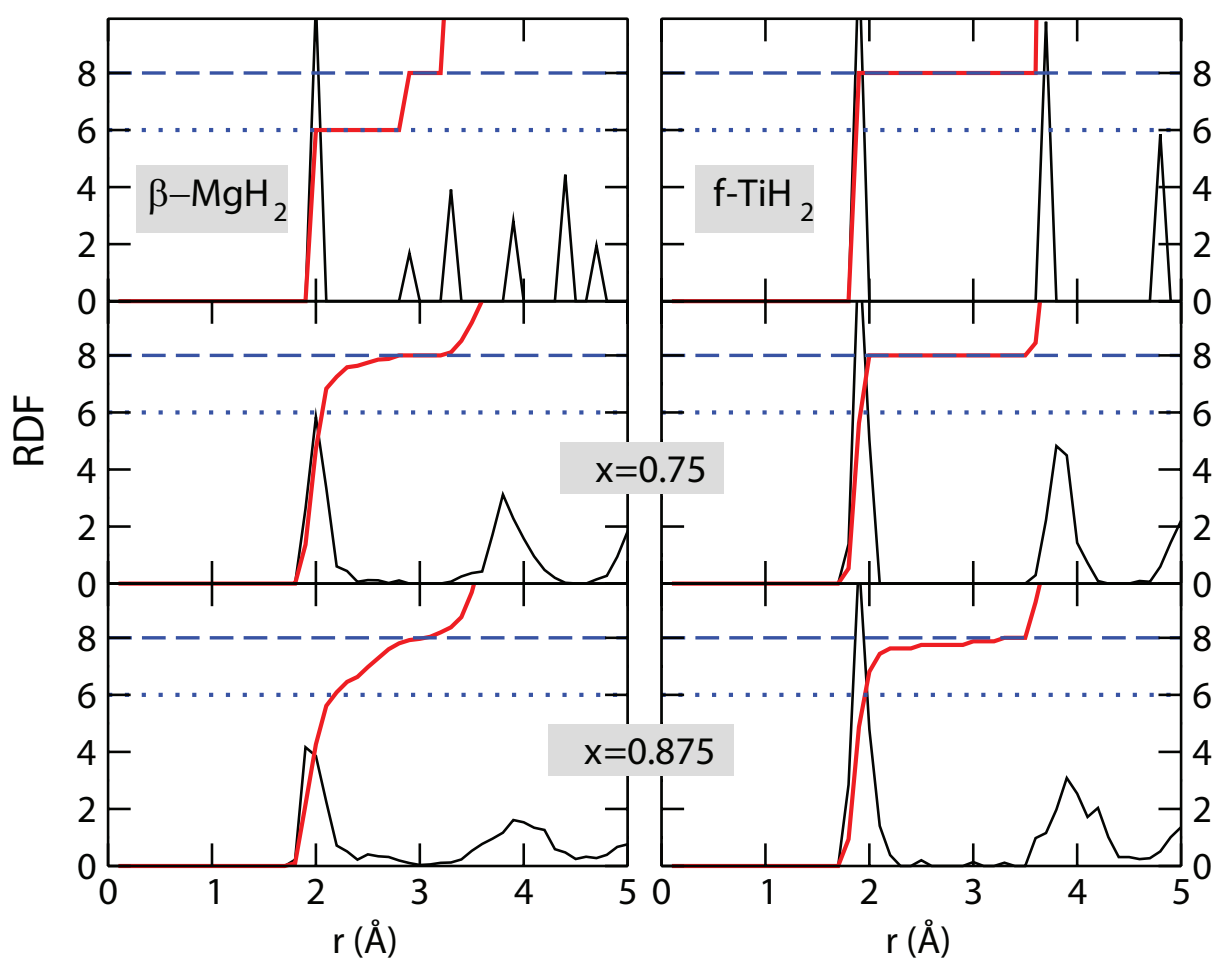

Figure 4.5: Radial distribution functions (RDFs) for $\mathrm{Mg}-\mathrm{H}$ (left column) and $\mathrm{Ti}-\mathrm{H}$ (right column) pairs in $\mathrm{Mg}_{x} \mathrm{Ti}_{(1-x)} \mathrm{H}_{2}, x=0.75,0.875$ fluoritetype SQSs, compared to the RDFs of $\beta-\mathrm{MgH}_{2}$ and $f-\mathrm{TiH}_{2}$. The red lines indicate the integrated RDF. Horizontal dotted and dashed lines indicate the $\mathrm{H}$ coordination numbers of $\mathrm{Mg}$ and $\mathrm{Ti}$ in the simple dihydrides, respectively.

supercells with a total number of atoms of 96 and 192, respectively. Optimizing the structure leads to some displacements in the positions of the metal atoms, but they remain close to fcc. There is a larger spread in the positions of the $\mathrm{H}$ atoms, which increases with increasing $x$. Representative examples of SQSs are given in figure 4.4 . Whereas in the ordered structures of table 4.4 the nearest neighbour $\mathrm{Ti}-\mathrm{Ti}$ distance increases with increasing $\mathrm{Mg}$ content $x$, in the SQSs one can always find at least one $\mathrm{Ti}-\mathrm{Ti}$ pair at a distance comparable to that in $f-\mathrm{TiH}_{2}$, for the compositions studied.

The optimized volumes of the SQSs are shown in figure 4.3. For $x \leq 0.75$ these volumes are within $1.5 \%$ of those of the ordered structures. Remarkably, the SQS volume of $\mathrm{Mg}_{0.875} \mathrm{Ti}_{0.125} \mathrm{H}_{2}$ is $4.9 \%$ larger than that of the ordered structure of table 4.4. This is indicative of a structural change at this composition, which can be 
clarified by calculating the radial distribution functions (RDFs) of the SQSs. The RDFs of $\mathrm{Mg}_{x} \mathrm{Ti}_{(1-x)} \mathrm{H}_{2}$ for $x=0.75,0.875$ are plotted in figure 4.5. Shown are the RDFs of $\mathrm{Ti}-\mathrm{H}$ and $\mathrm{Mg}-\mathrm{H}$ distances and the corresponding RDFs in the cubic $\beta$ $\mathrm{MgH}_{2}$ and $f-\mathrm{TiH}_{2}$ compounds. Integrating the RDF gives the number of hydrogen atoms surrounding each metal atom. The number in the first shell then corresponds to the coordination number of the metal atom.

In $f-\mathrm{TiH}_{2}$ each $\mathrm{Ti}$ atom is coordinated by eight $\mathrm{H}$ atoms at a distance of approximately $1.9 \AA$, whereas in the $\mathrm{Mg}$ atoms in $\beta-\mathrm{MgH}_{2}$ are coordinated by six $\mathrm{H}$ atoms at $2.0 \AA$, and by an additional two $\mathrm{H}$ atoms at $2.9 \AA$. The RDFs of the SQSs show that in $\mathrm{Mg}_{x} \mathrm{Ti}_{(1-x)} \mathrm{H}_{2}$ each $\mathrm{Ti}$ is coordinated as in $f-\mathrm{TiH}_{2}$, irrespective of the composition $x$. For $x=0.75$ each $\mathrm{Mg}$ atom is also coordinated by eight atoms at a similar distance, as in a fluorite structure. However, for $x=0.875$ the RDF for $\mathrm{Mg}-\mathrm{H}$ starts to resembles that of $\beta-\mathrm{MgH}_{2}$, i.e. a peak representing six $\mathrm{H}$ atoms at $\sim 2.0 \AA$, and a shoulder representing two $\mathrm{H}$ atoms at $\sim 2.9 \AA$. Apparently a large $\mathrm{Mg}$ content is required before such a structure can develop, which is accompanied by a volume expansion with respect to an ideal fluorite structure. In order not to complicate the terminology we call all such structures fluorite-type in the following.

Since we will compare the stability of these fluorite-type structures to rutile-type structures $\mathrm{Mg}_{x} \mathrm{Ti}_{(1-x)} \mathrm{H}_{2}$, we also make models of the latter using the $\alpha-\mathrm{MgH}_{2}$ structure as a starting point. To construct simple, ordered structures, we replace a fraction $x$ of the $\mathrm{Mg}$ atoms by TM atoms and use the smallest supercell of the rutile structure where this leads to an integer number of atoms. In case of multiple possible cells, the results given below refer to the cell that leads to the lowest energy. Rutile SQSs of $\mathrm{Mg}_{x} \mathrm{Ti}_{(1-x)}$ for $x=0.25-0.875$ are constructed using standard algorithms [50]. As it turns out that rutile structures are relatively unstable over a large composition range, we refrain from giving their structural details here.

\subsubsection{Stability}

Experimentally it is concluded that $\mathrm{Mg}$ and $\mathrm{Ti}$ do not form a thermodynamically stable bulk alloy. Indeed we find that its costs energy to make alloys from the bulk metals $\mathrm{Mg}$ and $\mathrm{Ti}$. For instance, the energy costs of making $\mathrm{Mg}_{x} \mathrm{Ti}_{(1-x)}$ fcc SQSs from bulk hcp $\mathrm{Mg}$ and Ti are 0.16 and $0.11 \mathrm{eV} /$ atom for $x=0.5$ and 0.75 , respectively, and for $\mathrm{Mg}_{x} \mathrm{Ti}_{(1-x)}$ in the simple, ordered $\mathrm{CuAu}$ and $\mathrm{Cu}_{3} \mathrm{Au}$ structures the energy costs are 0.22 and $0.15 \mathrm{eV} /$ atom, respectively. Calculations on hcp $\mathrm{Mg}_{x} \operatorname{Ti}_{(1-x)}$ decrease these numbers by $\sim 0.04 \mathrm{eV}$, which implies that the hcp alloy is more stable than the fcc alloy, but it is still unstable. Moreover, simple estimates of the configurational entropy show that the alloys cannot be stabilized at a reasonable 


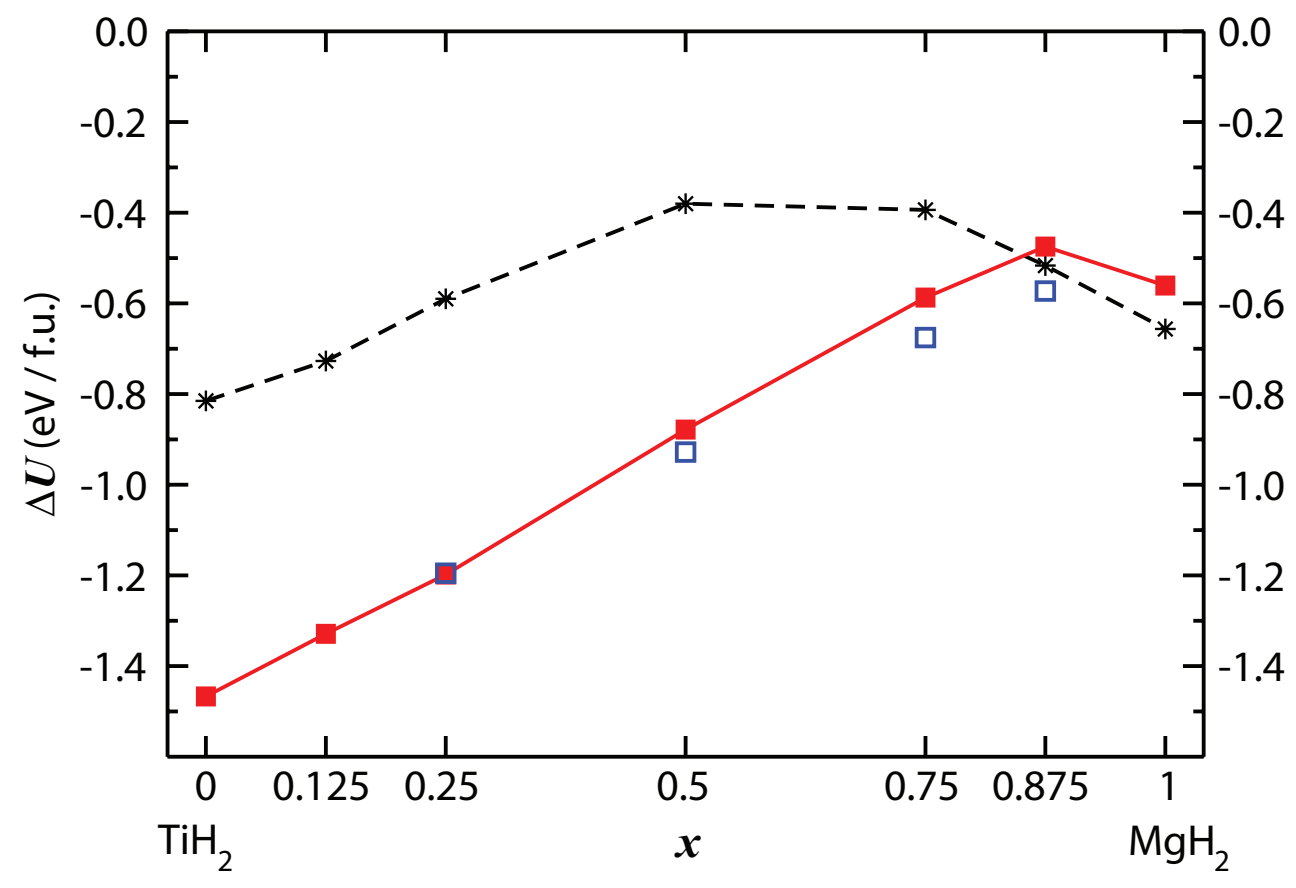

Figure 4.6: Formation energies of $\mathrm{Mg}_{x} \mathrm{Ti}_{(1-x)} \mathrm{H}_{2}$ as function of composition $x$ for ordered fluorite-type (filled red squares) and rutile-type (black stars) structures. The lines are added to guide the eye. The open blue squares indicate the formation energies of fluorite-type SQSs.

temperature by entropy. Metastable structures can still be relevant, though, as thin film deposition of $\operatorname{Mg}_{x} \operatorname{Ti}_{(1-x)}$ yields a hcp structure that survives a large number of (de)hydrogenation cycles.

The formation energy of $\mathrm{Mg}_{x} \mathrm{Ti}_{(1-x)} \mathrm{H}_{2}$ according to (4.1) is given in figure 4.6 for the fluorite and rutile structures. The numbers are given without ZPE corrections, which we will discuss later. Clearly all numbers are negative, which implies that the compounds are stable with respect to decomposition into the elements. In addition, over most of the composition range the fluorite structure is more stable than the rutile structure. For $x$ larger than a critical composition $x_{\mathrm{c}}$ the rutile structure becomes lower in energy. If we use the energies calculated for the simple ordered structures as a starting point and linearly interpolate between the compositions, we find $x_{\mathrm{c}} \approx 0.83$. The fact that $x_{\mathrm{c}}$ has a high value makes sense, since the energy difference between the $\alpha-\mathrm{MgH}_{2}$ (rutile) and $\beta-\mathrm{MgH}_{2}$ ("fluorite") phases is only 0.10 $\mathrm{eV} /$ f.u., whereas between $f-\mathrm{TiH}_{2}$ (fluorite) and $r-\mathrm{TiH}_{2}$ (rutile) it is $0.65 \mathrm{eV} /$ f.u.. 
Therefore, it requires a high $\mathrm{Mg}$ content to force the structure into rutile. Indeed, experimental results suggest that the cross-over from fluorite to rutile structures in $\mathrm{Mg}_{x} \mathrm{Ti}_{(1-x)} \mathrm{H}_{2}$ occurs for $x$ somewhere in the range 0.8-0.9 [15, 17].

Note that for all compositions the structures are metastable with respect to decomposition into $\mathrm{TiH}_{2}$ and $\mathrm{MgH}_{2}$, as is evident from figure 4.6. Evidently, the decomposition is kinetically hindered as it does not occur experimentally. Upon dehydrogenation the cubic $\mathrm{Mg}_{x} \mathrm{Ti}_{(1-x)} \mathrm{H}_{2}$ converts into hcp $\mathrm{Mg}_{x} \mathrm{Ti}_{(1-x)}$. Although the latter is metastable with respect to $\mathrm{Ti}$ and $\mathrm{Mg}$, its decomposition is also kinetically hindered, as thin films can be (de)hydrogenated reversibly. The apparent stability of these compounds is quite remarkable.

The formation energies of the fluorite SQSs are also given in figure 4.6. Within the constraints of the SQS approach, some variation in the relative ordering of $\mathrm{Ti}$ and $\mathrm{Mg}$ atoms is still possible at each composition $x$. We estimate that this variation gives a spread in the energies of $\sim 0.1 \mathrm{eV} /$ f.u.. From figure 4.6 one also observes that the most stable SQSs are slightly more stable than the ordered structures, by up to $\sim 0.1 \mathrm{eV} /$ f.u. for $x=0.875$. We have also constructed rutile SQSs of $\mathrm{Mg}_{x} \operatorname{Ti}_{(1-x)}$ for $x=0.25-0.875$. However, upon optimization these structures turn out to be unstable and spontaneously convert into a fluorite-type structure (i.e. using the conjugate gradient algorithm). This would suggest that a fluorite-type structure is stable at least up to a composition $x=0.875$. As the energies of the two types of structures are quite similar over a range of compositions close to the critical composition $x_{\mathrm{c}}$, the exact value of $x_{\mathrm{c}}$ is difficult to determine. Note that here we only consider simple ordered and SQSs models of rutile and flourite-type lattices. Of course, it cannot be excluded that different structural models describe the true ground state. However, our structures are judicially chosen so that our studies are relevant to understand the experiments; indeed we obtain a good agreement with experimental studies.

The ZPE corrections destabilize the simple hydrides $\alpha-\mathrm{MgH}_{2}$ and $\alpha-\mathrm{TiH}_{2}$, see table 4.2, and one expects this also to be the case in $\operatorname{Mg}_{x} \operatorname{Ti}_{(1-x)} \mathrm{H}_{2}$. The size of the ZPE correction in the simple hydrides indicates that it decreases with increasing $x$ from $0.19 \mathrm{eV} /$ f.u. at $x=0$ to $0.10 \mathrm{eV} /$ f.u. at $x=1$. We suggest that this decrease is approximately linear in $x$. An explicit calculation on the SQS of the $x=0.75$ composition gives a ZPE correction of $0.12 \mathrm{eV} /$ f.u., in agreement with this suggestion.

We now consider to what extend $\mathrm{Mg}_{x} \mathrm{Ti}_{(1-x)} \mathrm{H}_{2}$ is suitable as a hydrogen storage material. Experimentally it has been observed that the dehydrogenation kinetics becomes markedly slower if $x \gtrsim x_{0}=0.8$. The results of figure 4.6 strongly suggest that $x_{0}=x_{c}$, i.e. the composition at which the phase transition between fluorite 
and rutile structures takes place. Lightweight materials require a high content of magnesium, but to have a stable fluorite structure it should not exceed the critical composition $x_{c}$. We focus upon the composition $\mathrm{Mg}_{0.75} \mathrm{Ti}_{0.25} \mathrm{H}_{2}$, which should have the fluorite structure. It has gravimetric and volumetric hydrogen densities of $6.2 \mathrm{wt} \%$ and $0.135 \mathrm{~kg} / \mathrm{L}$, respectively. Its calculated formation energy is -0.59 $\mathrm{eV} /$ f.u. (without ZPE), which means that this compound is slightly less stable than $\alpha-\mathrm{MgH}_{2}$ with respect to decomposition into the elements. A decreased stability of the hydride should ease the dehydrogenation reaction. However, in order that the process is reversible, it is not advantageous if the alloy $\mathrm{Mg}_{x} \mathrm{Ti}_{(1-x)}$ dissociates upon dehydrogenation. If we calculate the hydrogenation energy according to the reaction

$$
\mathrm{Mg}_{x} \mathrm{Ti}_{(1-x)}+\mathrm{H}_{2}(g) \rightarrow \mathrm{Mg}_{x} \mathrm{Ti}_{(1-x)} \mathrm{H}_{2}
$$

we find $-0.76 \mathrm{eV} /$ f.u. for $x=0.75$. This number is in good agreement with the experimental value of $-0.81 \mathrm{eV} /$ f.u. of [73], obtained if the thin film correction suggested there is included. This hydrogenation enthalpy is somewhat lower than that of pure $\mathrm{Mg}$, showing that alloying $\mathrm{Mg}$ with $\mathrm{Ti}$ does not improve the thermodynamics as compared to pure $\mathrm{Mg}$. On the other hand, it is also not much worse, and the kinetics of (de)hydrogenation of $\mathrm{Mg}_{0.75} \mathrm{Ti}_{0.25} \mathrm{H}_{2}$ is markedly better than that of pure $\mathrm{Mg}$.

\subsubsection{Electronic structure and optical properties}

The calculated electronic density of states (DOS) of $\mathrm{Mg}_{x} \mathrm{Ti}_{(1-x)} \mathrm{H}_{2}$ in the simple ordered structures is shown in figure 4.7 for fluorite- and rutile-type structures. Qualitatively these DOSs can be interpreted as linear combinations of DOSs of the simple hydrides, see figure 4.2. In the DOSs of the rutile structure one can still distinguish the band gap that originates from $\mathrm{MgH}_{2}$. The states just above the gap originate from $\mathrm{Ti}$, as discussed in Sec. 4.4. In particular the DOS of rutile- $\mathrm{Mg}_{0.875} \mathrm{Ti}_{0.125} \mathrm{H}_{2}$ can be interpreted as Ti-doped $\alpha-\mathrm{MgH}_{2}$. Enlarging the Ti content then increasingly fills the gap with Ti-derived states and for rutile- $\mathrm{Mg}_{0.25} \mathrm{Ti}_{0.75} \mathrm{H}_{2}$, the DOS is dominated by a Ti-derived peak at the Fermi level.

A similar qualitative interpretation of the DOSs also holds for the $\operatorname{Mg}_{x} \operatorname{Ti}_{(1-x)} \mathrm{H}_{2}$ fluorite structures. It is somewhat less clear because the band gap in (fluorite-type) $\beta-\mathrm{MgH}_{2}$ is smaller than that of (rutile) $\alpha-\mathrm{MgH}_{2}$. Still the DOS of $\mathrm{Mg}_{0.875} \mathrm{Ti}_{0.125} \mathrm{H}_{2}$ can clearly be interpreted as Ti-doped $\beta-\mathrm{MgH}_{2}$, where Ti-derived states appear in the gap. Upon increasing the Ti content, the DOS more and more resembles that of $f-\mathrm{TiH}_{2}$ and the gap is completely filled. The latter has implications for the optical properties, as we will discuss below. 


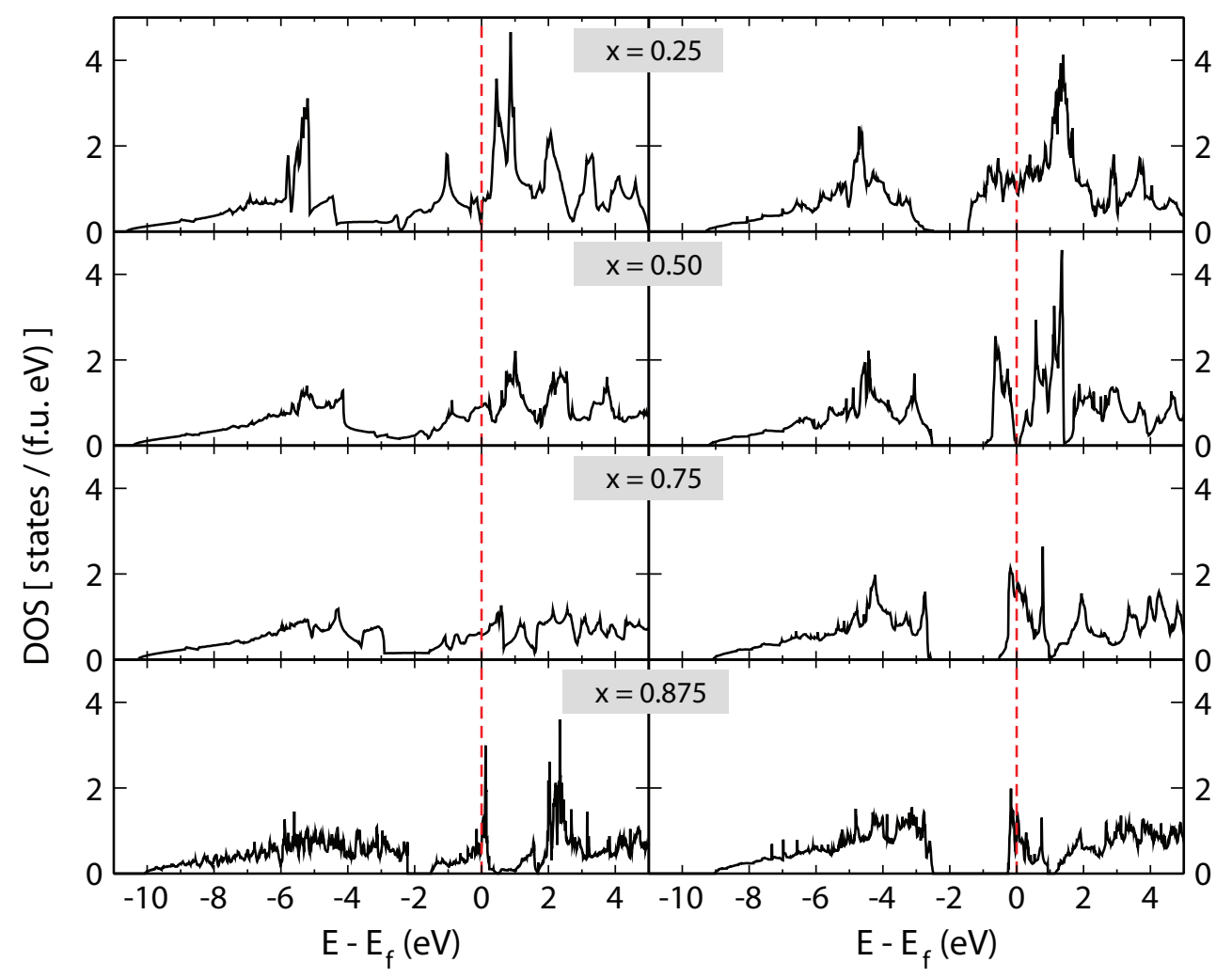

Figure 4.7: Electronic density of states (DOS) per formula unit for the fluorite (left column) and rutile (right column) $\mathrm{Mg}_{x} \mathrm{Ti}_{(1-x)} \mathrm{H}_{2}$ structures.

In order to be able to identify the character of the electronic states, the atom projected DOS of the simple structures is shown in fig. 4.8 for fluorite-type $\mathrm{Mg}_{x} \mathrm{Ti}_{(1-x)} \mathrm{H}_{2}$, $x=0.75,0.875$, which are the most relevant structures and compositions for studying the optical properties [15]. It is obvious that the peaks at the Fermi level are dominated by states of $\mathrm{Ti} 3 \mathrm{~d}$ character, as in $f-\mathrm{TiH}_{2}$, see figure 4.2. For $\mathrm{Mg}_{0.875} \mathrm{Ti}_{0.125} \mathrm{H}_{2}$ these peaks are narrow, reflecting the large distance of $6.62 \AA$ between neighbouring $\mathrm{Ti}$ atoms. One observes the $\mathrm{e}_{\mathrm{g}}-\mathrm{t}_{2 \mathrm{~g}}$ splitting that is typical of $\mathrm{d}$ states in a cubic crystal field. In this case the latter originates from the cubic coordination of each $\mathrm{Ti}$ atom by $\mathrm{H}$ atoms. Local magnetic moments of $1.5 \mu_{\mathrm{B}}$ develop on the Ti atoms and we predict an anti-ferromagnetic ordering of these moments at low temperature. The estimated Néel temperature is only 40K, however, demonstrating that the moments are atomic like. If we increase the $\mathrm{Ti}$ content, the states on neighbouring $\mathrm{Ti}$ atoms interact more strongly. For $\mathrm{Mg}_{0.75} \mathrm{Ti}_{0.25} \mathrm{H}_{2}$ the nearest neighbour distance between Ti atoms is $4.62 \AA$. The Ti $d$ derived peaks are much wider, and the local magnetic 


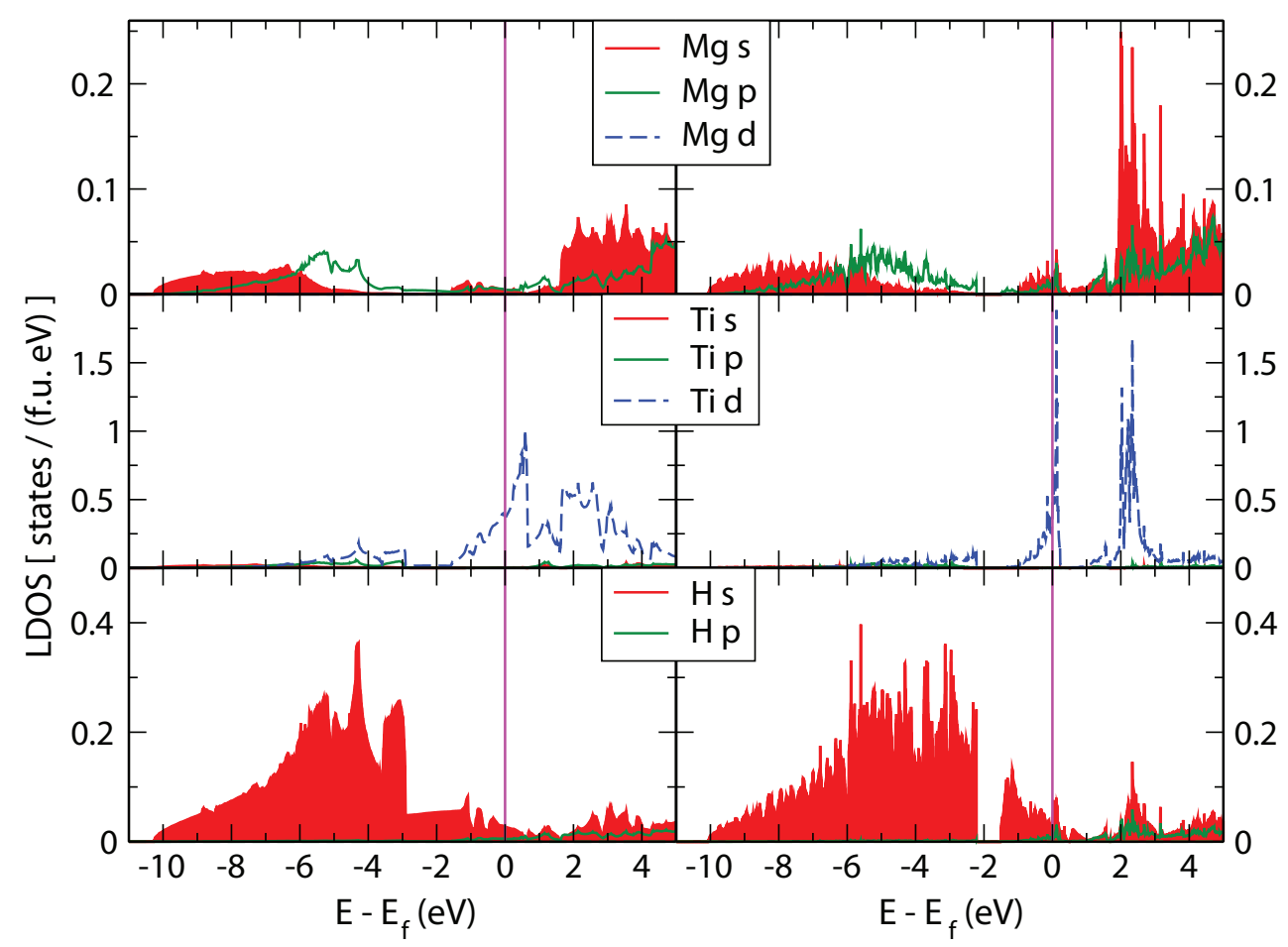

Figure 4.8: Local density of states (LDOS) for fluorite- $\mathrm{Mg}_{x} \mathrm{Ti}_{(1-x)} \mathrm{H}_{2}, x=$ 0.75 (left column) and $x=0.875$ (right column). The origin of the energy scale is set at the Fermi level. Atomic s, p and d contributions are shown in red, green and blue, respectively. The regions under the s curves are shaded and the d curves are dashed.

moments disappear.

The states in the PDOS at low energy are dominantly $\mathrm{H}$ 1s derived states, as expected. Interestingly, the states around the Fermi level also have a $\mathrm{H}$ contribution. These contributions are spread out over an energy range that is much wider in $\mathrm{Mg}_{x} \mathrm{Ti}_{(1-x)} \mathrm{H}_{2}$ than in the simple hydrides $\mathrm{MgH}_{2}$ and $\mathrm{TiH}_{2}$, see figure 4.2. The spread occurs in $\mathrm{H}$ derived bands of s character, as well as in the those of $\mathrm{p}$ character. Since transitions between such $\mathrm{H}$ derived bands give a large contribution to the optical response of a hydride [53, one might expect the response to be spread over a large energy range.

We calculate the optical response functions of $\mathrm{Mg}_{x} \mathrm{Ti}_{(1-x)} \mathrm{H}_{2}$ as described in Sec. 4.3. The refractive index $n(\omega)$ and the extinction coefficient $\kappa(\omega)$ are shown in figure 4.9 for the compositions $x=0.5,0.75$, and 0.875 . Most relevant for a com- 

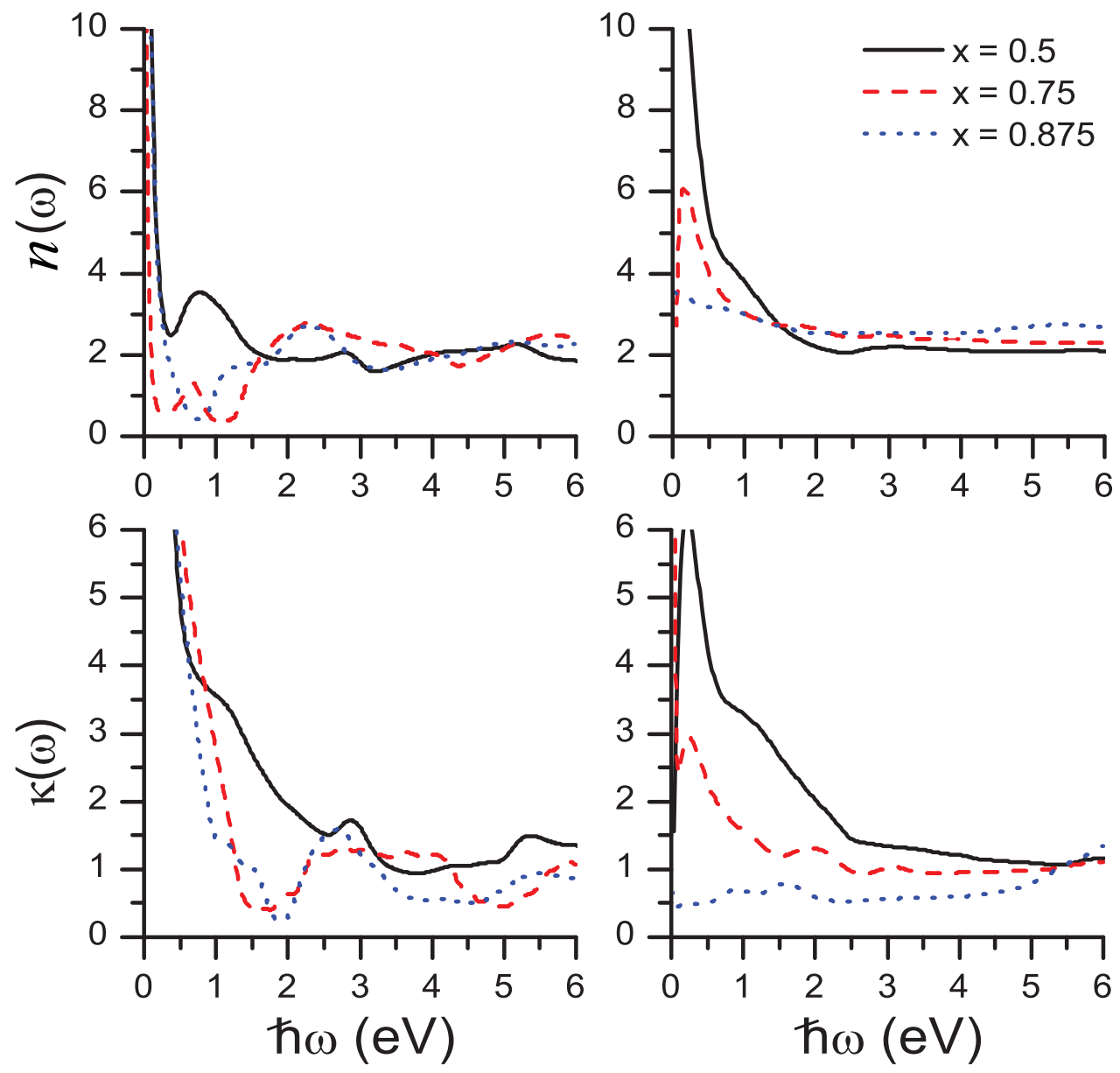

Figure 4.9: Index of refraction (top) $n(\omega)$ and extinction coefficient $\kappa(\omega)$ (bottom) of $\mathrm{Mg}_{x} \mathrm{Ti}_{(1-x)} \mathrm{H}_{2}$ as function of frequency $\omega$. The black (solid), red (dashed), and blue (dotted) lines are for the compositions $x=0.5,0.75,0.875$, respectively. The left and right columns are for the ordered structures and the SQSs, respectively.

parison to the optical experiments on thin films are the data for the SQSs. Comparing our data on the SQSs and the ordered structures, we observe that one main effect of disorder in the positions of the metal atoms, as modelled by a SQS, is smoothing the spectrum.

In addition, disorder strongly affects the lower end of the spectrum, i.e. $\omega=0$ $1.5 \mathrm{eV}$, in particular for the Mg-rich compositions $x=0.75,0.875$. The extinction coefficient $\kappa(\omega)$ of the SQSs in this frequency range is smaller than that of the ordered 
structures and much closer to its value at high frequency. The large $\kappa(\omega<1 \mathrm{eV})$ in the ordered structures is typical for the electron plasma response of metals, which matches the DOSs shown in figure 4.7. As the metallic properties of $\mathrm{Mg}_{x} \operatorname{Ti}_{(1-x)} \mathrm{H}_{2}$ originate from the Ti atoms only, introducing disorder in the Ti positions can destroy the electron plasma, suppressing the large $\kappa(\omega<1 \mathrm{eV})[26$. As can be observed in figure 4.9, compositions with a low Ti content are particularly sensitive to disorder, as the coupling between $\mathrm{Ti}$ atoms is relatively small.

Also the structure in the refractive index of the ordered structures at low frequency is suppressed in the SQSs and the refractive index at $\omega>1 \mathrm{eV}$ becomes close to constant. Assuming that one may use bulk optical functions to model the optical properties of $\mathrm{Mg}_{x} \mathrm{Ti}_{(1-x)} \mathrm{H}_{2}$, the reflectance (at normal incidence) of a surface is given by the usual Fresnel expression $R=\left((n-1)^{2}+\kappa^{2}\right) /\left((n+1)^{2}+\kappa^{2}\right)$. For a metal usually $\kappa \gg n$, giving rise to a large reflectance. In this case both $\kappa$ and $n$ are rather moderate for frequencies in the visible region. Using the data shown in figure 4.9 one obtains an almost constant low reflectance $R \approx 0.2$ over the whole visible region. Since the extinction coefficient is also almost constant in the visible region and it has a quite considerable value, the material $\mathrm{Mg}_{x} \mathrm{Ti}_{(1-x)} \mathrm{H}_{2}$ has a dark, colourless, i.e. black appearance.

Using the extinction coefficient of the $\mathrm{Mg}_{x} \mathrm{Ti}_{(1-x)} \mathrm{H}_{2}$ SQSs shown in figure 4.9 to calculate the absorption $\alpha(\omega)=2 \kappa(\omega) \omega / c$, we obtain a good qualitative agreement with experimental data [15]. The quantitative difference between the calculated and experimental absorption is $\sim 30 \%$, which reflects both the approximations made in the calculations, and in the extraction of the experimental values.

\subsection{Summary and Conclusions}

$\mathrm{MgH}_{2}$ has a high hydrogen storage capacity, but it suffers from poor (de) hydrogenation kinetics and a high thermodynamic stability, which make it unsuitable as a hydrogen storage material. Adding $\mathrm{Ti}$ to $\mathrm{Mg}$ gives an alloy hydride $\mathrm{Mg}_{x} \mathrm{Ti}_{(1-x)} \mathrm{H}_{2}$ with much faster (de)hydrogenation kinetics for $x \lesssim 0.8$. In this paper we have studied the structure and stability of $\mathrm{Mg}_{x} \mathrm{Ti}_{(1-x)} \mathrm{H}_{2}, x=0-1$, by first-principles calculations at the level of density functional theory.

As the most stable structures (at ambient conditions) of $\mathrm{MgH}_{2}$ and $\mathrm{TiH}_{2}$ are rutile and fluorite, respectively, we model such structures for the alloy hydride $\mathrm{Mg}_{x} \mathrm{Ti}_{(1-x)} \mathrm{H}_{2}$. A series of simple, ordered fluorite structures is constructed analogous to the high pressure $\mathrm{Mg}_{7} \mathrm{TiH}_{16}$ phase, and rutile structures are modelled in $\mathrm{MgH}_{2}$ supercells. All cell parameters and atomic positions are optimized and care is 
taken to converge the total energies with respect to computational parameters such as the basis set size and the $k$-point sampling grid. Upon optimization, all structures stay quite close to either fluorite or rutile. To model disorder in the positions of the metal atoms, we construct a series of SQSs of $\mathrm{Mg}_{x} \mathrm{Ti}_{(1-x)} \mathrm{H}_{2}$ starting from fluorite and rutile structures. Upon optimization the fluorite structures are stable, but in compositions with a high $\mathrm{Mg}$ content, such as $\mathrm{Mg}_{0.875} \mathrm{Ti}_{0.125} \mathrm{H}_{2}$, the structure around the $\mathrm{Mg}$ atoms starts to resemble that of the high pressure phase $\beta-\mathrm{MgH}_{2}$. The SQS rutile structures of $\mathrm{Mg}_{x} \mathrm{Ti}_{(1-x)} \mathrm{H}_{2}$ are unstable for $x \leq 0.875$. From the formation energies of the ordered, as well as the disordered structures, we give evidence for a fluorite to rutile phase transition at a critical composition $x_{\mathrm{c}}=0.8-0.9$. This correlates with the experimentally observed sharp decrease in (de)hydrogenation rates around this composition.

Thin films of $\mathrm{Mg}_{x} \mathrm{Ti}_{(1-x)}$ show a remarkable optical transition from reflecting to absorbing upon hydrogenation, which is reversible upon dehydrogenation. We calculate the optical response of $\mathrm{Mg}_{x} \mathrm{Ti}_{(1-x)} \mathrm{H}_{2}, x=0.5-0.875$, in the ordered and disordered structures and show that the black absorbing state is an intrinsic property of this material. $\mathrm{Mg}_{x} \mathrm{Ti}_{(1-x)} \mathrm{H}_{2}$ has a peak at the Fermi level for all compositions $x$, which is composed of Ti d states. However, the resulting metallic plasma only plays a minor role in the optical response in the visible range for compositions with a high $\mathrm{Mg}$ content, as interband transitions interfere to decrease the extinction coefficient $\kappa$. Moreover, this effect is enhanced by disorder in the positions of the Ti atoms, which easily destroys the metallic plasma and suppresses the optical reflection even more. The contributions of the $\mathrm{H}$ atoms to the bands are spread out over a large energy range as a result of the diverse coordination by $\mathrm{Ti}$ and $\mathrm{Mg}$ atoms in $\mathrm{Mg}_{x} \mathrm{Ti}_{(1-x)} \mathrm{H}_{2}$. Interband transitions then result in an almost constant optical absorption over a large energy range, causing the black appearance of these compounds.

\section{Acknowledgments}

The authors thank G. Kresse and J. Harl (Universität Wien) for the use of the optical packages, and A. V. Ruban (Technical University of Denmark, Lyngby) for his help and for making available his program to generate quasi random structures. This work is part of the research programs of "Advanced Chemical Technologies for Sustainability (ACTS)" and the "Stichting voor Fundamenteel Onderzoek der Materie (FOM)". The use of supercomputer facilities was sponsored by the "Stichting Nationale Computerfaciliteiten (NCF)". These institutions are financially supported by "Nederlandse Organisatie voor Wetenschappelijk Onderzoek (NWO)". 


\section{Bibliography}

[1] See the special issue Toward a Hydrogen Economy, by R. Coontz and B. Hanson, Science 305, 957 (2004).

[2] A. Züttel, Mater. Today 6, 24 (2003).

[3] L. Schlapbach and A. Züttel, Nature 414, 353 (2001).

[4] A. Züttel, Naturwiss. Unterr. Chem. 91, 157 (2004).

[5] J. Huot, G. Liang, and R. Schulz, Appl. Phys. A: Mater. Sci. Process. 72, 187 (2001).

[6] L. Schlapbach, editor, Hydrogen in Intermetallic Compounds II (Springer, Berlin, 1992).

[7] F. Manchester and D. Khatamian, Mater. Sci. Forum 31, 261 (1988).

[8] A. Zaluska, L. Zaluski, and J. O. Ström-Olsen, J. Alloys Compd. 288, 217 (1999).

[9] A. Zaluska, L. Zaluski, and J. O. Ström-Olsen, Appl. Phys. A: Mater. Sci. Process. 72, 157 (2001).

[10] R. A. H. Niessen and P. H. L. Notten, Electrochem. Solid-State Lett. 8, A534 (2005).

[11] R. A. H. Niessen and P. H. L. Notten, J. Alloys Compd. 404, 457 (2005).

[12] P. Vermeulen, R. A. H. Niessen, and P. H. L. Notten, Electrochem. Commun. 8, 27 (2006).

[13] G. Liang, J. Huot, S. Boily, A. V. Neste, and R. Schulz, J. Alloys Compd. 292, 247 (1999).

[14] M. Latroche, W. P. Kalisvaart, and P. H. L. Notten, J. Solid State Chem. 179, 3024 (2006).

[15] D. Borsa et al., Phys. Rev. B 75, 205408 (2007).

[16] P. C. M. M. Magusin, W. P. Kalisvaart, P. H. L. Notten, and R. A. van Santen, Chem. Phys. Lett. 456, 55 (2008).

[17] P. Vermeulen et al., J. Mater. Chem. 18, 3680 (2008). 
[18] P. Vermeulen et al., Electrochem. Solid-State Lett. 9, A520 (2006).

[19] D. M. Borsa et al., Appl. Phys. Lett. 88, 241910 (2006).

[20] M. Slaman et al., Sens. Actuators: B 123, 538 (2007).

[21] A. Baldi et al., Int. J. Hydrogen Energy 33, 3188 (2008).

[22] D. Kyoi et al., J. Alloys Compd. 372, 213 (2004).

[23] E. Rönnebro, D. Kyoi, A. Kitano, Y. Kitano, and T. Sakai, J. Alloys Compd. 404, 68 (2005).

[24] S. Er, D. Tiwari, G. A. de Wijs, and G. Brocks, Phys. Rev. B 79, 024105 (2009).

[25] B. R. Pauw et al., Acta Mater. 56, 2948 (2008).

[26] M. J. van Setten, S. Er, G. Brocks, R. A. de Groot, and G. A. de Wijs, Phys. Rev. B 79, 125117 (2009).

[27] T. J. Richardson et al., Appl. Phys. Lett. 78, 3047 (2001).

[28] W. Lohstroh et al., Phys. Rev. Lett. 93, 197404 (2004).

[29] P. E. Blöchl, Phys. Rev. B 50, 17953 (1994).

[30] G. Kresse and D. Joubert, Phys. Rev. B 59, 1758 (1999).

[31] G. Kresse and J. Hafner, Phys. Rev. B 47, 558 (1993).

[32] G. Kresse and J. Furthmüller, Phys. Rev. B 54, 11169 (1996).

[33] G. Kresse and J. Furthmüller, Comput. Mater. Sci. 6, 15 (1996).

[34] J. P. Perdew and Y. Wang, Phys. Rev. B 45, 13244 (1992).

[35] A. D. Becke, J. Chem. Phys. 98, 5648 (1993).

[36] J. Heyd, G. E. Scuseria, and M. Ernzerhof, J. Chem. Phys. 118, 8207 (2003).

[37] J. Paier et al., J. Chem. Phys. 124, 154709 (2006).

[38] J. Paier et al., J. Chem. Phys. 125, 249901 (2006).

[39] J. Paier, M. Marsman, and G. Kresse, J. Chem. Phys. 127, 024103 (2007).

[40] S. Froyen, Phys. Rev. B 39, 3168 (1989). 
[41] M. C. Payne, M. P. Teter, D. C. Allan, T. A. Arias, and J. D. Joannopoulos, Rev. Mod. Phys. 64, 1045 (1992).

[42] M. Methfessel and A. T. Paxton, Phys. Rev. B 40, 3616 (1989).

[43] P. E. Blöchl, O. Jepsen, and O. K. Andersen, Phys. Rev. B 49, 16223 (1994).

[44] K. P. Huber and G. Herzberg, Molecular Spectra and Molecular Structure (Van Nostrand Reinhold, New York, 1979).

[45] H. Hemmes, A. Driessen, and R. Griessen, J. Phys. C: Solid State Phys. 19, 3571 (1986).

[46] M. J. van Setten, G. A. de Wijs, M. Fichtner, and G. Brocks, Mater. Chem. 20, 4952 (2008).

[47] M. J. van Setten, G. A. de Wijs, and G. Brocks, Phys. Rev. B 77, 165115 (2008).

[48] G. Kresse, J. Furthmüller, and J. Hafner, Europhys. Lett. 32, 729 (1995).

[49] A. Zunger, S. H. Wei, L. G. Ferreira, and J. E. Bernard, Phys. Rev. Lett. 65, 353 (1990).

[50] A. V. Ruban, S. I. Simak, S. Shallcross, and H. L. Skriver, Phys. Rev. B 67, 214302 (2003).

[51] C. Wolverton, V. Ozolins, and A. Zunger, J. Phys.: Condens. Matter 12, 2740 (1999).

[52] M. Gajdoš, K. Hummer, G. Kresse, J. Furthmüller, and F. Bechstedt, Phys. Rev. B 73, 045112 (2006).

[53] M. J. van Setten, V. A. Popa, G. A. de Wijs, and G. Brocks, Phys. Rev. B 75, 35204 (2007).

[54] J. N. Huiberts et al., Nature 380, 231 (1996).

[55] P. van Gelderen, P. A. Bobbert, P. J. Kelly, and G. Brocks, Phys. Rev. Lett. 85, 2989 (2000).

[56] P. van Gelderen, P. A. Bobbert, P. J. Kelly, G. Brocks, and R. Tolboom, Phys. Rev. B 66, 075104 (2002).

[57] M. J. van Setten, G. A. de Wijs, V. A. Popa, and G. Brocks, Phys. Rev. B 72, 073107 (2005). 
[58] M. J. van Setten, G. A. de Wijs, and G. Brocks, Phys. Rev. B 76, 075125 (2007).

[59] J. Harl, G. Kresse, L. D. Sun, M. Hohage, and P. Zeppenfeld, Phys. Rev. B 76, 35436 (2007).

[60] K. Momma and F. Izumi, IUCr Newslett. 7, 106 (2006).

[61] P. Villars, L. D. Calvert, and W. B. Pearson, Pearson's Handbook of Crystallographic Data for Intermetallic Phases (ASM International, Materials Park, OH, USA, 1991).

[62] M. Bortz, B. Bertheville, G. Bottger, and K. Yvon, J. Alloys Compd. 287, L4 (1999).

[63] J. P. Bastide, B. Bonnetot, J. M. Letoffe, and P. Claudy, Mater. Res. Bull. 15, 1215 (1980).

[64] P. Vajeeston, P. Ravindran, A. Kjekshus, and H. Fjellvåg, Phys. Rev. Lett. 89, 175506 (2002).

[65] F. D. Manchester, Phase Diagrams of Binary Hydrogen Alloys (ASM International, Materials Park, OH, USA, 2000).

[66] B. Bogdanovic et al., J. Alloys Compd. 282, 84 (1999).

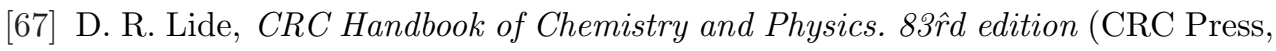
Boca Raton, 2002).

[68] R. Griessen and T. Riesteter, Topics in Applied Physics-Hydrogen in Intermetallic Compounds (Springer, Berlin, 1988).

[69] J. Isidorsson, I. Giebels, H. Arwin, and R. Griessen, Phys. Rev. B 68, 115112 (2003).

[70] M. R. Hartman, J. J. Rush, T. J. Udovic, R. C. Bowman, and S. J. H. Jr., J. Solid State Chem. 180, 1298 (2007).

[71] J. Hafner, J. Phys. F: Met. Phys. 15, L43 (1985).

[72] G. Liang and R. Schulz, J. Mater. Sci. 38, 1179 (2003).

[73] R. Gremaud et al., Adv. Mater. 19, 2813 (2007). 


\section{Chapter 5}

\section{A computational study of hydrogen storage in Mg-Ti-X $(\mathrm{X}=\mathrm{Al}, \mathrm{Si})$}

\subsection{Abstract}

Mixing Mg with Ti leads to an alloy with markedly improved hydrogenation kinetics as compared to pure $\mathrm{Mg}$. However, the resulting hydrides are too stable for applications as hydrogen storage materials at moderate temperatures and pressures. Moreover Mg-Ti alloys are unstable with respect to decomposition into the elements. In this paper we examine by means of first-principles density functional theory calculations whether adding $\mathrm{Al}$ or $\mathrm{Si}$ to $\mathrm{Mg}$-Ti can improve its thermodynamic properties. Indeed we find that $\mathrm{Mg}-\mathrm{Ti}-\mathrm{X}(\mathrm{X}=\mathrm{Al}$ or $\mathrm{Si})$ alloys are more stable than $\mathrm{Mg}$-Ti alloys, whereas their hydrides are less stable. Controlling the structure of $\mathrm{Mg}$-Ti-X alloys brings the hydrogenation energy in a range that is suitable for their use as hydrogen storage materials.

\subsection{Introduction}

Future use of hydrogen as an energy carrier depends on finding safe, compact and cost effective methods for storing hydrogen[1]. Solid state storage in the form of metal hydrides is promising since it offers both a safe way of handling hydro- 
gen, as well as high volumetric hydrogen densities 2, 3]. Attaining high gravimetric hydrogen densities using solid state storage methods is limited to compounds comprised of lightweight elements. Magnesium, an inexpensive and abundant metal, can store up to 7.65 wt.\% hydrogen [4. The application of $\mathrm{MgH}_{2}$ as a hydrogen storage material is however obstructed by its high thermodynamic stability and very low (de)hydrogenation rates, both of which lead to excessively high desorption temperatures $T \gtrsim 300^{\circ}[5]$. A significant amount of research has been devoted to reduce the stability of $\mathrm{MgH}_{2}$ or to enhance its (de)hydrogenation kinetics. Experiments on thin films have shown that alloying magnesium with early transition metal (TM) elements can markedly improve the hydrogen kinetics [6 9. The best results have been obtained with the TMs $\mathrm{Sc}$ and $\mathrm{Ti}$ and alloy compositions $\mathrm{Mg}_{(1-x)} \mathrm{TM}_{x}$ with $x \approx 0.2$.

The improved kinetics have been linked to the existence of a cubic, fluorite-type $\mathrm{Mg}_{(1-x)} \mathrm{TM}_{x} \mathrm{H}_{2}$ phase, whereas the tetragonal rutile phase of $\mathrm{MgH}_{2}$ would hamper the (de)hydrogenation kinetics. From first principles calculations we have shown that a fluorite-type phase of $\mathrm{Mg}_{(1-x)} \mathrm{TM}_{x} \mathrm{H}_{2}$ is stable with respect to a rutile-type phase for $\mathrm{TM}=\mathrm{Sc}, \mathrm{Ti}, \mathrm{V}$ and $\mathrm{Cr}$, and $x \gtrsim 0.2$ [10]. However, the dehydrogenation enthalpy of these compounds is either larger, or at least not significantly smaller, than that of $\mathrm{MgH}_{2}$, implicating that the thermodynamic properties are not improved (sufficiently). Moreover, the stability of the $\mathrm{Mg}_{(1-x)} \mathrm{TM}_{x}$ alloys decreases along the series $\mathrm{Sc}, \mathrm{Ti}, \mathrm{V}, \mathrm{Cr}$. In fact, only $\mathrm{Mg}_{(1-x)} \mathrm{Sc}_{x}$ is thermodynamically stable with respect to decomposition into the elemental metals ( $\mathrm{Mg}$ and $\mathrm{Sc}$ ). Scandium is a rare element, however, which prevents its large scale use in a hydrogen storage material. $\mathrm{Mg}_{(1-x)} \mathrm{V}_{x}$ and $\mathrm{Mg}_{(1-x)} \mathrm{Cr}_{x}$ are thermodynamically quite unstable, which hampers their use in a reversible system, which needs to undergo repeated hydrogen (un)loading cycles.

$\mathrm{Ti}$ is an abundant element and thus suitable for large scale applications. The binary phase diagram of $\mathrm{Mg}$ and $\mathrm{Ti}$ indicates that a stable alloy between these two elements cannot be formed. Studies of kinetically stabilized bulk Mg-Ti samples have been inconclusive [11 13, but $\mathrm{Mg}$-Ti alloys are readily formed in thin films, which are stable under repeated (de)hydrogenation cycles 6, , 8, 9, 11, 14-16. The high stability of Mg-Ti hydride remains a problem, however. The calculated dehydrogenation enthalpy of $\mathrm{Mg}_{0.75} \mathrm{Ti}_{0.25} \mathrm{H}_{2}$ is $0.76 \mathrm{eV} / \mathrm{H}_{2}$, which is close to the values reported for $\mathrm{MgH}_{2}$. Recently, Vermeulen et al. [17] have reported that incorporating $\mathrm{Al}$ or $\mathrm{Si}$ into $\mathrm{Mg}$-Ti thin films decreases the dehydrogenation energy. The hydrogen storage capacity is a maximum $6 \mathrm{wt}$. \% for a composition consisting of 70 at.\% Mg, 20 at.\% $\mathrm{Ti}$ and 10 at.\% Al. Similarly, 4.53 wt. \% hydrogen is stored in a Mg-Ti-Si alloy of a 
similar composition.

In this paper we calculate the stability of $\mathrm{Mg}$-Ti-X $(\mathrm{X}=\mathrm{Al}$ or $\mathrm{Si})$ alloys and their hydrides for several possible compositions by means of first-principles density functional theory (DFT) calculations. Simultaneous deposition of the metals very likely results in random alloy thin films, whereas by controlled subsequent deposition one might form multilayer structures. We study the energetics of examples of both disordered structures and multilayers.

\subsection{Computational methods}

DFT calculations are performed at the level of the generalized gradient approximation (GGA) using the PW91 functional [18. We employ the projector augmented wave (PAW) technique and a plane wave basis set [19, 20], as implemented in the Vienna $A b$ initio Simulation Package (VASP) 21-23. Standard frozen core PAW potentials are used, where the $\mathrm{H} 1 s, \mathrm{Mg} 3 s, \mathrm{Al} 3 s 3 p$, Si $3 s 3 p$ and Ti $3 p 4 s 4 d$ shells are treated as valence shells. As Ti has a partially filled $3 d$ shell, the possibility of spin-polarization is examined. The plane wave kinetic energy cutoff is set at $518 \mathrm{eV}$. The Brillouin zone is integrated using a regular $\mathbf{k}$-point mesh with a spacing of 0.02 $\AA^{-1}$ and the Methfessel-Paxton scheme with a smearing parameter of $0.1 \mathrm{eV}[24$. The volumes and shapes of the unit cells are optimized, as well as the positions of the atoms. Total energies of the optimized structures are finally calculated using the tetrahedron method [25]. Details on the optimization procedure can be found in Ref. [26].

\subsection{Mg-Ti-Al,Si metals}

The aim of adding $\mathrm{Al}$ or $\mathrm{Si}$ is to stabilize the metal $\mathrm{Mg}-\mathrm{Ti}$, which otherwise is thermodynamically unstable, as discussed above. Al and $\mathrm{Si}$ are chosen because both $\mathrm{Mg}$ and $\mathrm{Ti}$ form binary alloys with $\mathrm{Al}$ and Si. We first look at the energetics of these binary alloys, and then study the possibilities of forming ternary alloys, either as disordered structures, or as multilayers.

\subsubsection{Binary alloys}

The elements $\mathrm{Mg}$ and $\mathrm{Ti}$ crystallize in the hcp structure, whereas $\mathrm{Al}$ and $\mathrm{Si}$ adopt the cubic fcc and diamond structures, respectively. The binary phase diagram of $\mathrm{Mg}$ and $\mathrm{Al}$ indicates that alloys between these elements can be formed at various 
Table 5.1: Optimized lattice parameters ( $a$ and $c / a$ ) and formation energies with respect to the elemental solids for $\mathrm{MX}(\mathrm{M}=\mathrm{Mg}, \mathrm{Ti} ; \mathrm{X}=\mathrm{Al}, \mathrm{Si})$ in the $L 1_{0}$ structure.

\begin{tabular}{lrrr}
\hline \hline Compound & $a(\AA)$ & $c / a$ & $E_{f}(\mathrm{eV} /$ atom $)$ \\
\hline $\mathrm{MgAl}$ & 4.2802 & 0.9838 & -0.015 \\
$\mathrm{MgSi}$ & 4.2084 & 0.9400 & 0.099 \\
$\mathrm{TiAl}$ & 3.9918 & 1.0201 & -0.409 \\
$\mathrm{TiSi}$ & 3.8932 & 0.9836 & -0.495 \\
\hline \hline
\end{tabular}

compositions. With regards to hydrogen storage properties, experimental research shows that the hydrogen plateau pressure maximizes if the $\mathrm{Mg}$ content equals the $\mathrm{Al}$ content in the samples [27]. $\mathrm{Mg}$ and $\mathrm{Si}$ form a stable compound $\mathrm{Mg}_{2} \mathrm{Si}$ with a cubic fluorite structure, where Si atoms occupy the corners and faces of a fcc cell and $\mathrm{Mg}$ atoms are in the tetrahedral interstitial positions [28]. It is well-known, however, that the formation of $\mathrm{Mg}_{2} \mathrm{Si}$ inhibits the absorption of hydrogen [29. The binary phase diagrams of $\mathrm{Ti}$ and $\mathrm{Al}$ or $\mathrm{Si}$ show several stable phases. The most stable alloys are obtained if the amount of $\mathrm{Ti}$ is equal to that of $\mathrm{Al}$ or $\mathrm{Si}$ [17, 30, 31].

With an eye on possible ternary Mg-Ti-X compounds, it should be noted that the solid solubility of $\mathrm{Ti}$ in $\mathrm{Al}$ or $\mathrm{Si}$ is much higher that of $\mathrm{Ti}$ in $\mathrm{Mg}$ [17. Therefore, it might be expected that in a stable structure of Mg-Ti-X Ti is surrounded by $\mathrm{Al}$ or Si. TiAl crystalizes in a tetragonally distorted fcc structure with space group P4/mmm (123), the so-called $L 1_{0}$ structure [31. TiSi forms a similar $L 1_{0}$ structure [30]. Both compounds are very stable, as illustrated by their calculated formation energies shown in Table 5.1. The formation energies per atom are calculated as

$$
E_{f}=\left[E_{\mathrm{MX}}-E_{\mathrm{M}}-E_{\mathrm{X}}\right] / n
$$

where $E_{\mathrm{MX}}, E_{\mathrm{M}}$ and $E_{\mathrm{X}}$ are total energies of the $\mathrm{MX}$ alloys $(\mathrm{M}=\mathrm{Mg}$ or $\mathrm{Ti}, \mathrm{X}=$ $\mathrm{Al}$ or $\mathrm{Si}$ ) in the $L 1_{0}$ structure, and the bulk metals $\mathrm{M}$ and $\mathrm{X}$ in their ground state structures, respectively; $n$ is the number of atoms in the unit cell. Table 5.1 also shows the results for $\mathrm{MgX}$ in the $L 1_{0}$ structure, confirming that TiX is indeed much more stable than the corresponding $\mathrm{MgX}$. Yet $\mathrm{MgAl}$ and $\mathrm{MgSi}$ are more stable than $\mathrm{MgTi}$, which indicates that adding $\mathrm{Al}$ or $\mathrm{Si}$ to a $\mathrm{Mg}$-Ti alloy can stabilize it.

If one adds $\mathrm{Si}$ to $\mathrm{Mg}$ - Ti, the formation of $\mathrm{Mg}_{2} \mathrm{Si}$ needs to be prevented, since the latter compound does not absorb hydrogen [29]. We consider the reaction

$$
\mathrm{Mg}_{2} \mathrm{Si}+\mathrm{Ti} \longleftrightarrow \mathrm{TiSi}+2 \mathrm{Mg}
$$




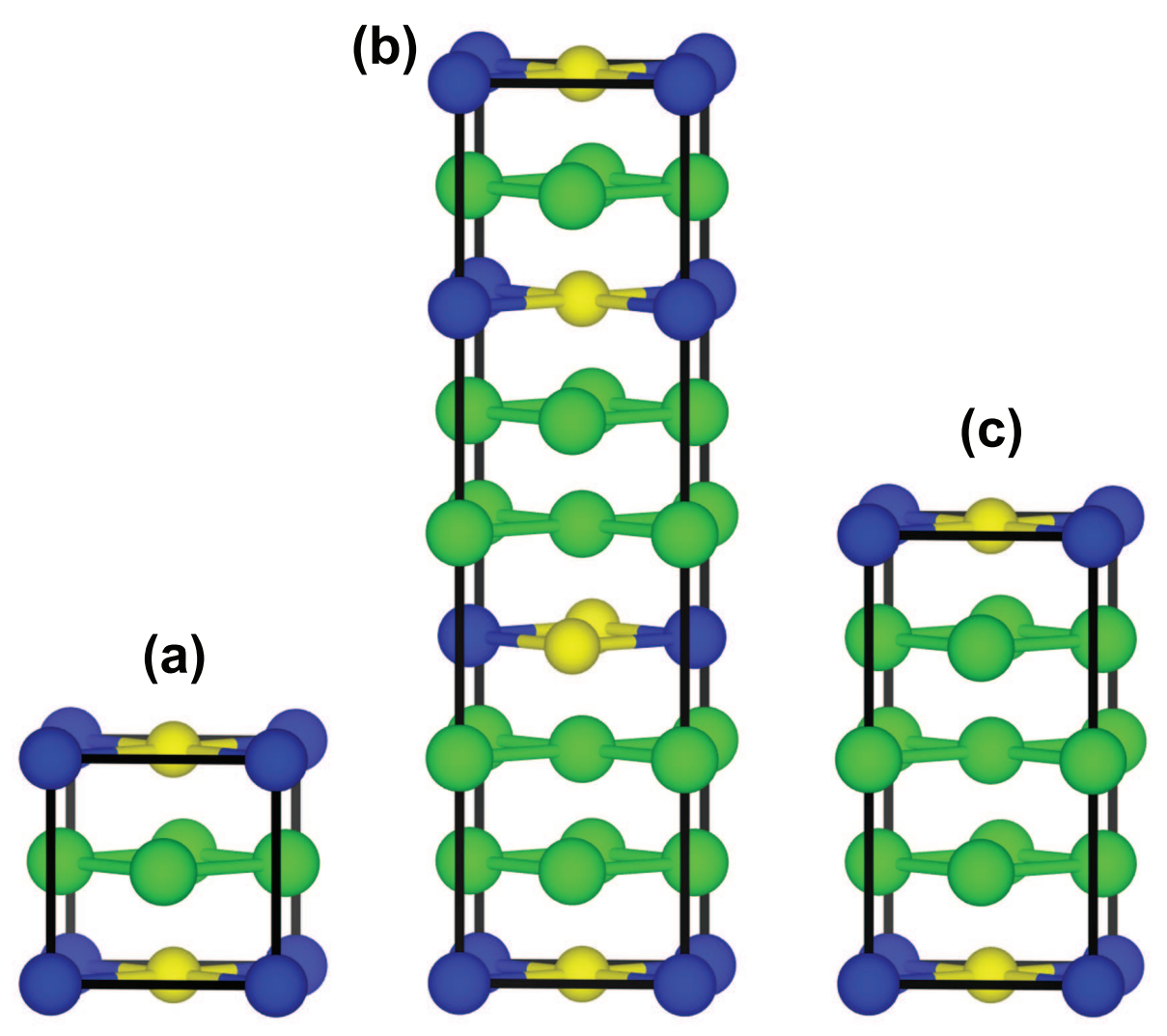

Figure 5.1: (Color online) Layered $\mathrm{Mg}_{(1-2 y)} \mathrm{Ti}_{y} \mathrm{X}_{y}$ structures for (a) $y=$ 0.25 , (b) 0.1875 and (c) 0.125 compositions are shown. $\mathrm{Mg}$, Ti and $\mathrm{X}$ (= Al or $\mathrm{Si}$ ) atoms are shown as green, blue and yellow colored spheres, respectively. All structural drawings are produced using the VESTA software [32.

with $\mathrm{Mg}_{2} \mathrm{Si}$ in the fluorite structure and TiSi in the $L 1_{0}$ structure. Total energy calculations show that the materials on the right hand side of Eq. (5.2) are more stable by $0.35 \mathrm{eV}$ per TiSi unit. Therefore, one can design the Mg-Ti-Si compound such, that the Ti captures the $\mathrm{Si}$ and prevents the formation of $\mathrm{Mg}_{2} \mathrm{Si}$.

\subsubsection{Mg-Ti-Al,Si layered structures}

In view of the stability of TiAl and TiSi it makes sense to look at ternary Mg-Ti-X alloys whose Ti:X ratio is fixed at 1:1, i.e. $\mathrm{Mg}_{(1-2 y)} \operatorname{Ti}_{y} \mathrm{X}_{y}$. In particular we study the compositions $y=0.125,0.1875$, and 0.25 , which cover the experimentally reported 
Table 5.2: The calculated formation energies with respect to the elemental solids of $\mathrm{Mg}_{(1-2 y)} \mathrm{Ti}_{y} \mathrm{X}_{y}$ in the layered structures, see Fig. 5.1 .

\begin{tabular}{lrrr}
\hline \hline & \multicolumn{3}{c}{$E_{f}(\mathrm{eV} /$ atom $)$} \\
\cline { 2 - 4 } Compound & $\mathrm{X}=\mathrm{Ti}$ & $\mathrm{X}=\mathrm{Al}$ & $\mathrm{X}=\mathrm{Si}$ \\
\hline $\mathrm{Mg}_{0.5} \mathrm{Ti}_{0.25} \mathrm{X}_{0.25}$ & 0.206 & -0.083 & -0.132 \\
$\mathrm{Mg}_{0.625} \mathrm{Ti}_{0.1875} \mathrm{X}_{0.1875}$ & 0.141 & -0.015 & -0.040 \\
$\mathrm{Mg}_{0.75} \mathrm{Ti}_{0.125} \mathrm{X}_{0.125}$ & 0.093 & 0.010 & 0.006 \\
\hline \hline
\end{tabular}

range of compositions that show interesting hydrogen storage properties [17.

We first look at relatively simple structures, which are based upon alternating atomic layers of $\mathrm{TiX}$ and $\mathrm{Mg}$. One could envision such multilayers being formed by atomic beam epitaxy, for instance. Starting from the assumption that the atoms occupy fcc positions, the composition $\mathrm{Mg}_{0.5} \mathrm{Ti}_{0.25} \mathrm{X}_{0.25}$ can be formed by alternating in the (001) direction one layer of $\mathrm{TiX}$ and one layer of $\mathrm{Mg}$, as shown in Fig. 5.1. The composition $\mathrm{Mg}_{0.75} \mathrm{Ti}_{0.125} \mathrm{X}_{0.125}$ can then be formed by alternating one layer of $\mathrm{TiX}$ with three layers of $\mathrm{Mg}$. Following this strategy, the composition $\mathrm{Mg}_{0.625} \mathrm{Ti}_{0.1875} \mathrm{X}_{0.1875}$ then becomes a more complex alternation of one TiX layer and one or two Mg layers. The resulting unit cells are shown in Fig. 5.1.

The formation energies per atom of these $\mathrm{Mg}_{(1-2 y)} \mathrm{Ti}_{y} \mathrm{X}_{y}$ structures are given in Table 5.2. They can be compared to the formation energies of layered $\mathrm{Mg}_{(1-2 y)} \mathrm{Ti}_{2 y}$ compounds with the same amount of $\mathrm{Mg}$. Mg-Ti is quite unstable over the whole composition range with respect to phase separation into the elements. The formation energy of these layered Mg-Ti structures scales linearly with the Ti content, which one may attribute to the formation of Mg-Ti interfaces. It gives an interface energy of $0.45 \mathrm{eV}$ per interface and atom. Adding $\mathrm{Al}$ or Si indeed increases the stability, as can be observed from the formation energies of $\mathrm{Mg}_{(1-2 y)} \mathrm{Ti}_{y} \mathrm{X}_{y}$.

\subsubsection{Mg-Ti-Al,Si disordered structures}

In the experiments so far, all three metals are deposited simultaneously [17. As the structures are not known from experiment and a full computational search for the structure of a ternary alloy is computationally prohibitive, we start from the following assumptions. We position all metal atoms at fcc sites. Starting from $\mathrm{MgTi}$ we assume that there is no correlation between the positions of the $\mathrm{Mg}$ and the Ti atoms. Such random structures can be modeled in finite supercells by special quasirandom structures (SQSs) 33 35. We use supercells containing 32 metal atoms 
(a)

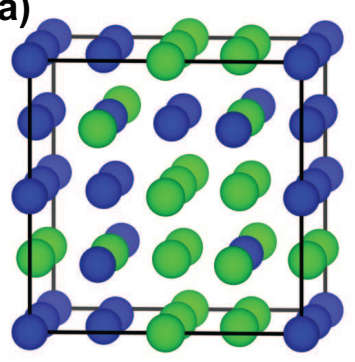

(b)

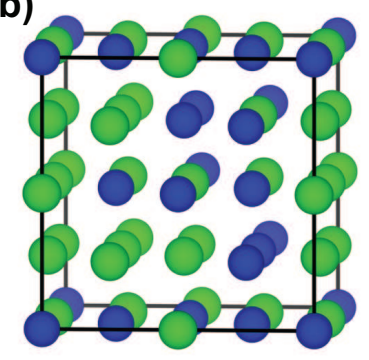

(c)

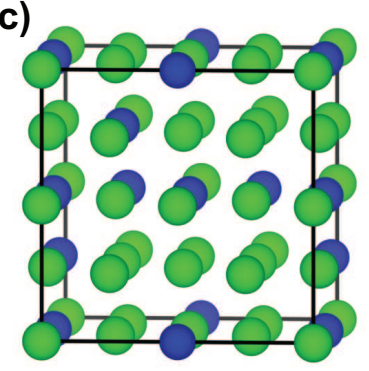

Figure 5.2: (Color online) Prototype Mg-Ti SQSs containing (a) 50, (b) 37.5 and (c) 25 at. \% Ti are shown. Mg and Ti atoms are shown as green and blue colored spheres, respectively.

to model SQSs of $\mathrm{Mg}_{(1-x)} \operatorname{Ti}_{x}, x=0.25,0.375,0.5$, and optimize their structure and cell parameters. Examples of such structures are given in Fig. 5.2 .

Adding a third element in the composition $\mathrm{Mg}_{(1-2 y)} \mathrm{Ti}_{y} \mathrm{X}_{y}$ means that half of the Ti atoms should be replaced by $\mathrm{X}$ atoms, where $\mathrm{X}=\mathrm{Al}$ or Si. From the discussion in the previous sections one might guess that $\mathrm{X}$ atoms have a preference to be bonded to $\mathrm{Ti}$, rather than $\mathrm{Mg}$ atoms. To examine whether there is such a preference, the coordination numbers $N_{\mathrm{Ti}}$ of each Ti by Ti atoms in the nearest neighbor shells, are calculated for the SQSs of $\mathrm{Mg}_{(1-x)} \operatorname{Ti}_{x}$. If we replace a Ti atom with a large $N_{\mathrm{Ti}}$ by an $\mathrm{X}$ atom, the latter will then be surrounded mainly by nearest neighbor Ti atoms, whereas replacing a $\mathrm{Ti}$ atom with a small $N_{\mathrm{Ti}}$ results in the $\mathrm{X}$ atom being surrounded mainly by $\mathrm{Mg}$ atoms.

We order the Ti atoms in the SQSs of $\mathrm{Mg}_{(1-x)} \mathrm{Ti}_{x}$ according to their $N_{\mathrm{Ti}}$ values and consider three scenarios for replacing half of them by $\mathrm{X}$ atoms. In the first scenario Ti atoms with the largest $N_{\mathrm{Ti}}$ are replaced, which leads a maximum coordination of $\mathrm{X}$ atoms by $\mathrm{Ti}$ or $\mathrm{X}$ atoms. We call this the high coordination $(H)$ structure in the following. In contrast, in the second scenario only Ti atoms with small $N_{\mathrm{Ti}}$ are replaced, giving $\mathrm{X}$ atoms that are mostly surrounded by $\mathrm{Mg}$ atoms, which we call low $(L)$ coordination. The third scenario is a case of mixed $(M)$ coordination, where half of the replaced Ti atoms have a large $N_{\mathrm{Ti}}$ and half of them have a small $N_{\mathrm{Ti}}$. This $M$ structure mainly serves as a check on the results obtained for the $L$ and $M$ cases. It should be noted that in the $L(H)$ structure the Ti atoms that remain in $\mathrm{Mg}_{(1-2 y)} \operatorname{Ti}_{y} \mathrm{X}_{y}$ all have a large (small) $N_{\mathrm{Ti}}$.

The formation energies with respect to the elements of the $\mathrm{Mg}_{(1-2 y)} \operatorname{Ti}_{y} \mathrm{X}_{y}$ structures constructed via these three scenarios are presented in Table 5.3. They can be 
Table 5.3: Calculated formation energies, $E_{f}$ (eV/atom), with respect to the elemental solids of $\mathrm{Mg}_{(1-2 y)} \mathrm{Ti}_{y} \mathrm{X}_{y}$ in the disordered structures. The construction of the $L, H$, and $M$ structures is discussed in the text.

\begin{tabular}{|c|c|c|c|c|c|c|c|}
\hline \multirow[b]{2}{*}{ Compound } & \multirow[t]{2}{*}{$\mathrm{X}=\mathrm{Ti}$} & \multicolumn{3}{|c|}{$\mathrm{X}=\mathrm{Al}$} & \multicolumn{3}{|c|}{$\mathrm{X}=\mathrm{Si}$} \\
\hline & & $L$ & $H$ & $M$ & $L$ & $H$ & $M$ \\
\hline $\mathrm{Mg}_{0.5} \mathrm{Ti}_{0.25} \mathrm{X}_{0.25}$ & 0.156 & -0.008 & 0.052 & 0 & -0.066 & 0.006 & -0.035 \\
\hline $\mathrm{Mg}_{0.625} \mathrm{Ti}_{0.1875} \mathrm{X}_{0.1875}$ & 0.153 & 0.025 & 0.091 & 0.046 & -0.020 & 0.064 & 0.020 \\
\hline $\mathrm{Mg}_{0.75} \mathrm{Ti}_{0.125} \mathrm{X}_{0.125}$ & 0.110 & 0.035 & 0.058 & 0.063 & 0.038 & 0.058 & 0.064 \\
\hline
\end{tabular}

compared to the formation energies of the corresponding $\mathrm{Mg}(1-2 y) \mathrm{Ti}_{2 y}$ structures. Apparently, $\mathrm{Al}$ and $\mathrm{Si}$ both have a stabilizing effect on the Mg-Ti compounds, irrespective of where these atoms are positioned, with Si having a larger effect than $\mathrm{Al}$. Moreover, the stability of $\mathrm{Mg}_{(1-2 y)} \mathrm{Ti}_{y} \mathrm{X}_{y}$ increases with increasing $y$. However, on an absolute scale only the structures with $y=0.25$ for $\mathrm{Al}$ and $y=0.25,0.1875$ for $\mathrm{Si}$ are thermodynamically stable with respect to decomposition into the elements.

Comparing the $L, H$, and $M$ structure in Table 5.3 one observes that the relative ordering of the metal atoms can have a sizable effect on the formation energies. Perhaps somewhat contra-intuitively the $L$ case yields the lowest energy, whereas the $H$ case gives the highest energy. In the $L$ structures the $\mathrm{Al}$ or $\mathrm{Si}$ atoms have a small $N_{\mathrm{Ti}}$, meaning that they are mainly surrounded by $\mathrm{Mg}$ atoms, which in view of Table 5.1 does not seem to give much stabilization. However, the Ti atoms that remain in the $L$ structure all have a large $N_{\mathrm{Ti}}$, which means that they are mainly surrounded by $\mathrm{Ti}$ atoms. This minimizes the number of direct Mg-Ti contacts that decrease the stability. Thus the maximum beneficial effect on the formation energy of adding $\mathrm{X}(=\mathrm{Al}, \mathrm{Si})$ in these structures is not so much in forming close $\mathrm{Ti}-\mathrm{X}$ contacts, but in preventing direct Mg-Ti contacts. Since the Ti atoms in the $H$ structure all have a small $N_{\mathrm{Ti}}$, the number of $\mathrm{Mg}$-Ti contacts is large, and the energy of this structure is higher that of the $L$ structure. The energies of the $M$ structures are between that of the $L$ and $H$ structures, which is in line with this analysis. An exception to this rule is the composition $\mathrm{Mg}_{0.75} \mathrm{Ti}_{0.125} \mathrm{X}_{0.125}$. Here however the $\mathrm{Mg}$ content is so large that the spread in $N_{\mathrm{Ti}}$ is not large, which makes the distinction between sites with large and small $N_{\mathrm{Ti}}$ a bit arbitrary.

Comparing the formation energies of the layered structures, Table [5.2] to those of the disordered structures, Table 5.3 , one observes that the layered structures are more stable. This may provide new ideas for structures with interesting properties, formed by immiscible elements. 
Table 5.4: Calculated hydrogenation energies $E_{h}\left(\mathrm{eV} / \mathrm{H}_{2}\right)$ of $\mathrm{Mg}_{(1-2 y)} \mathrm{Ti}_{y} \mathrm{X}_{y} \mathrm{H}_{2}$ in the layered and the disordered $L$ structures. The second and the fifth column represent the formation energies of the corresponding $\mathrm{Mg}_{(1-2 y)} \mathrm{Ti}_{2 y} \mathrm{H}_{2}$ structures.

\begin{tabular}{lcccccccc}
\hline \hline & \multicolumn{3}{c}{ layered } & & \multicolumn{4}{c}{ disordered $L$} \\
\cline { 2 - 3 } \cline { 7 - 8 } Compound & $\mathrm{X}=\mathrm{Ti}$ & $\mathrm{X}=\mathrm{Al}$ & $\mathrm{X}=\mathrm{Si}$ & & $\mathrm{X}=\mathrm{Ti}$ & $\mathrm{X}=\mathrm{Al}$ & $\mathrm{X}=\mathrm{Si}$ \\
\hline & & & & & & & & \\
$\mathrm{Mg}_{0.5} \mathrm{Ti}_{0.25} \mathrm{X}_{0.25} \mathrm{H}_{2}$ & -1.118 & -0.178 & 0.335 & & -1.120 & -0.358 & -0.156 \\
$\mathrm{Mg}_{0.625} \mathrm{Ti}_{0.1875} \mathrm{X}_{0.1875} \mathrm{H}_{2}$ & -0.906 & -0.278 & 0.093 & & -0.981 & -0.468 & -0.240 \\
$\mathrm{Mg}_{0.75} \mathrm{Ti}_{0.125} \mathrm{X}_{0.125} \mathrm{H}_{2}$ & -0.711 & -0.317 & -0.086 & & -0.807 & -0.501 & -0.434 \\
\hline \hline
\end{tabular}

\subsection{Mg-Ti-Al,Si Hydrides}

In this section we discuss the energetics of $\mathrm{Mg}$-Ti-Al,Si hydrides. In previous studies we have shown that a fluorite-type cubic structure of $\mathrm{Mg}_{(1-x)} \mathrm{Ti}_{x} \mathrm{H}_{2}$ is more stable than the $\mathrm{MgH}_{2}$ rutile-type structure for $x \gtrsim 0.1-0.2$ [10, 26]. In the following we assume that replacing half of the $\mathrm{Ti}$ atoms by $\mathrm{Al}$ or $\mathrm{Si}$ maintains the fluorite structure. Experimentally the (de)hydrogenation kinetics of $\mathrm{Mg}-\mathrm{Ti}-\mathrm{X}(=\mathrm{Al}, \mathrm{Si})$ is comparably fast as that of $\mathrm{Mg}-\mathrm{Ti}$, which is attributed to the existence of a fluorite structure [17. We use the structural models of the Mg-Ti-X alloys discussed in the previous sections and position hydrogen atoms in the tetrahedral interstitial sites to obtain fluorite-type $\mathrm{Mg}_{(1-2 y)} \mathrm{Ti}_{y} \mathrm{X}_{y} \mathrm{H}_{2}$ structures. For the disordered systems we use the $L$ structures, since these give the lowest energies for the alloys. Subsequently all cell parameters and geometries of these hydrides are optimized.

We calculate the hydrogenation energies according to

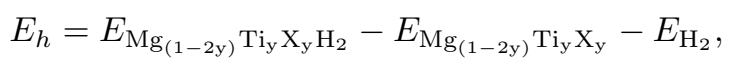

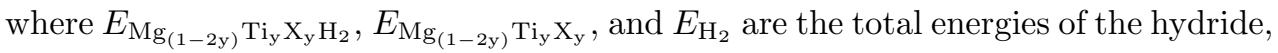
the corresponding alloy, and the hydrogen molecule, respectively. The calculated hydrogenation energies are given in Table 5.4 .

The results clearly show that the hydrides $\mathrm{Mg}_{(1-2 y)} \mathrm{Ti}_{y} \mathrm{X}_{y} \mathrm{H}_{2}$ are much less stable than their corresponding $\mathrm{Mg}_{(1-2 y)} \mathrm{Ti}_{2 y} \mathrm{H}_{2}$, whether in the simple, layered structures, or in the disordered structures. Since the alloys $\mathrm{Mg}_{(1-2 y)} \mathrm{Ti}_{y} \mathrm{X}_{y}$ are more stable than their corresponding $\mathrm{Mg}_{(1-2 y)} \mathrm{Ti}_{2 y}$, see Tables 5.2 and 5.3 this is an example of van Mal's rule [36]. The latter states that stabilization of an alloy phase is accompanied by a destabilization of its hydride. Van Mal's rule also holds if one compares the layered structures to the disordered structures. The layered structures of the $\mathrm{Mg}_{(1-2 y)} \mathrm{Ti}_{y} \mathrm{X}_{y}$ 
alloys are more stable then their corresponding disordered structures, compare Tables 5.2 and 5.3. One then expects the hydrides $\mathrm{Mg}_{(1-2 y)} \mathrm{Ti}_{y} \mathrm{X}_{y} \mathrm{H}_{2}$ to be less stable in the layered structures than in the disordered structures, as indeed can be observed in Table 5.4. Moreover, since Mg-Ti-Si alloys are more stable than Mg-Ti-Al alloys, one may expect that Mg-Ti-Si hydrides are less stable than Mg-Ti-Al hydrides. The results in Table 5.4 confirm this.

We now consider the hydrogenation energies in a more quantitative way. A hydrogenation energy of $\sim-0.4 \mathrm{eV} / \mathrm{H}_{2}$ gives an equilibrium hydrogen pressure of $\sim 1$ bar at room temperature [2, 3. Even with this hydrogenation energy the amount of heat released in a very short time when a bulk material is loaded with hydrogen, can be a problem in some applications. Therefore, depending on the application a hydrogenation energy between $\sim-0.15$ and $\sim-0.4 \mathrm{eV} / \mathrm{H}_{2}$ is required. Starting with the pure Mg-Ti alloys one observes that their hydrogenation energies are far outside this range. They are in fact larger or comparable to the hydrogenation energy of pure $\mathrm{Mg}[10,26]$.

The addition of $\mathrm{Al}$ or Si markedly destabilizes the hydrides and brings the hydrogenation energies into the range required for possible applications, where Si has a larger effect than Al. The effects are particularly large in the layered structures. In fact, $\mathrm{Mg}_{(1-2 y)} \mathrm{Ti}_{y} \mathrm{Si}_{y} \mathrm{H}_{2}$ in a layered structure is unstable, or marginally stable for the compositions we studied. In contrast, the hydrogenation energy of $\mathrm{Mg}_{(1-2 y)} \mathrm{Ti}_{y} \mathrm{Al}_{y}$ is $\sim-0.3 \mathrm{eV} / \mathrm{H}_{2}$. Moreover, the alloy $\mathrm{Mg}_{(1-2 y)} \mathrm{Ti}_{y} \mathrm{Al}_{y}$ is stable with respect to decomposition into the elements for $y=0.1875,0.25$, and only marginally unstable for $y=0.125$, see Table 5.2. These properties indicate that $\mathrm{Mg}_{(1-2 y)} \mathrm{Ti}_{y} \mathrm{Al}_{y}$ in layered form is potentially a promising material for hydrogen storage.

The layered structures of $\mathrm{Mg}_{(1-2 y)} \mathrm{Ti}_{y} \mathrm{X}_{y} \mathrm{H}_{2}$ are relatively simple. Hydrogen atoms occupy positions at, or close to the tetrahedral interstitial sites, as one would expect of a fluorite structure, see Fig. 5.3. Such an atomic arrangement could be advantageous for fast hydrogen kinetics.

Also in the disordered structures does the addition of $\mathrm{Al}$ or $\mathrm{Si}$ destabilize the hydrides, although the effects are not as large as in the layered structures. Again $\mathrm{Si}$ has a larger effect than $\mathrm{Al}$, but the hydrogenation energies of all compositions studied are markedly better than those of pure $\mathrm{Mg}$, or $\mathrm{Mg}-\mathrm{Ti}$, in accordance with the experimental findings [17. If one demands that the $\mathrm{Mg}_{(1-2 y)} \mathrm{Ti}_{y} \mathrm{X}_{y}$ alloys are stable with respect to decomposition into the elements, then for $\mathrm{X}=\mathrm{Al}$, Si, one needs $y=0.25$ and $y=0.1875,0.25$, respectively, see Table 5.3, which gives hydrogenation energies ranging from -0.22 to $-0.37 \mathrm{eV} / \mathrm{H}_{2}$.

The optimized structures of the disordered $L$ structures are shown in Fig. 5.4. The 


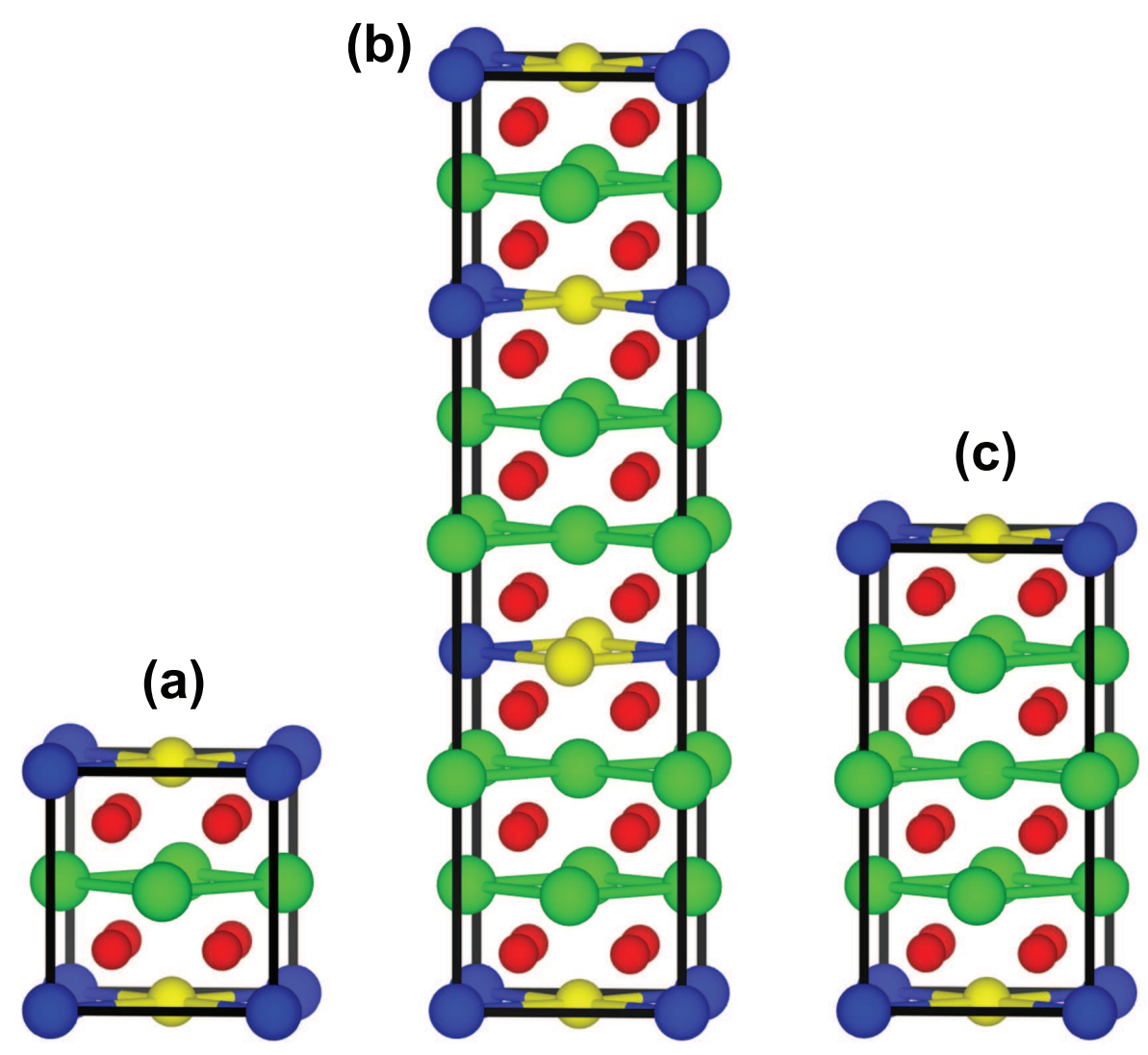

Figure 5.3: (Color online) Optimized structures of $\mathrm{Mg}_{(1-2 y)} \mathrm{Ti}_{y} \mathrm{Al}_{y} \mathrm{H}_{2}$ in layered form. Hydrogen atoms are shown as red colored spheres. The compositions with (a) $y=0.25$, (b) 0.1875 , and (c) 0.125 are shown from left to right.

top row shows Mg-Ti-X compounds with decreasing Ti content from left to right. The hydrogen atoms stay close to the tetrahedral interstitial sites in almost every case, which makes these structures fluorite-like. The largest deviations are observed for the composition with the lowest Ti content (or highest Mg content). The hydrogens close to the $\mathrm{Mg}$ atoms then start to assume positions that are typical for the cubic $\beta$-phase of $\mathrm{MgH}_{2}$, as is explained in detail in Ref. [26]. In a fluorite $\mathrm{MH}_{2}$ structure each metal atom $\mathrm{M}$ is cubically coordinated by eight hydrogen atoms. This is perfect if $\mathrm{M}=\mathrm{Ti}$ ( $\mathrm{TiH}_{2}$ has the fluorite structure), but $\mathrm{Mg}$ prefers a octahedral coordination (which occurs both in rutile $\alpha-\mathrm{MgH}_{2}$, and cubic $\beta-\mathrm{MgH}_{2}$ ). 

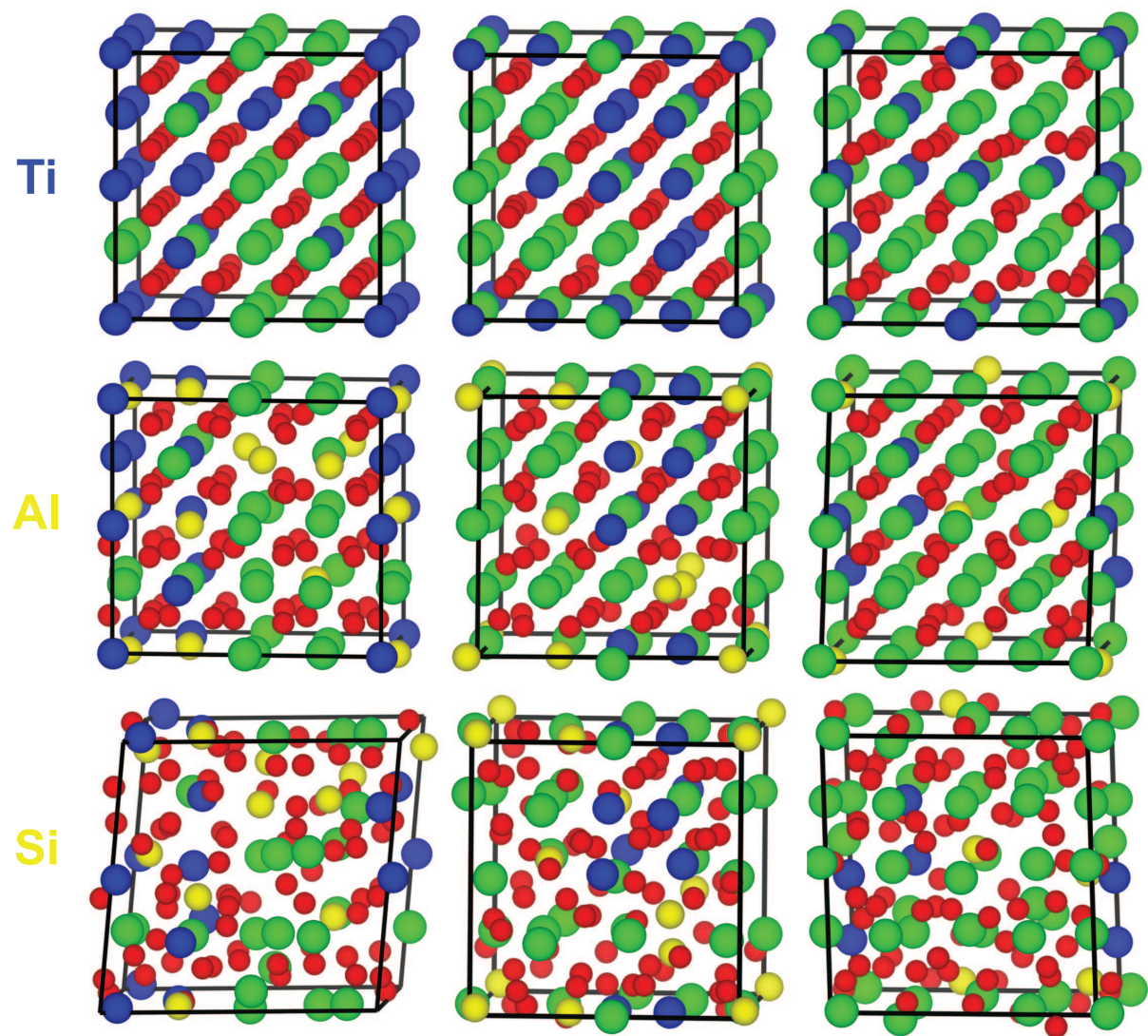

$y=$

0.25

0.1875

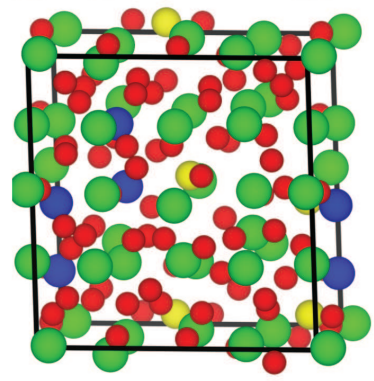

0.125

Figure 5.4: (Color online) Optimized structures of $\mathrm{Mg}_{(1-2 y)} \mathrm{Ti}_{y} \mathrm{X}_{y} \mathrm{H}_{2}$ in the disordered $L$ structure. The columns from left to right are for the compositions $y=0.25,0.1875$, and 0.125 , respectively. The rows from top to bottom are for $\mathrm{X}=\mathrm{Ti}, \mathrm{Al}$, and $\mathrm{Si}$, respectively.

The middle and bottom rows of Fig. 5.4 show the fully optimized structures of $\mathrm{Mg}_{(1-2 y)} \mathrm{Ti}_{y} \mathrm{Al}_{y} \mathrm{H}_{2}$ and $\mathrm{Mg}_{(1-2 y)} \mathrm{Ti}_{y} \mathrm{Si}_{y} \mathrm{H}_{2}$, respectively, for the different compositions. It is clear that the positions of the hydrogen atoms are noticeably shifted from the tetrahedral interstitial sites. Both $\mathrm{Mg}$ and $\mathrm{Al}$ prefer an octahedral coordination by hydrogen atoms, which is different from the cubic coordination found in a fluorite structure. Si prefers a tetrahedral coordination, which explains why the structure of $\mathrm{Mg}_{(1-2 y)} \mathrm{Ti}_{y} \mathrm{Si}_{y} \mathrm{H}_{2}$ deviates substantially from a fluorite-type structure. 


\section{Acknowledgments}

This work is part of the research programs of "Advanced Chemical Technologies for Sustainability (ACTS)" and the "Stichting voor Fundamenteel Onderzoek der Materie (FOM)". The use of supercomputer facilities was sponsored by the "Stichting Nationale Computerfaciliteiten (NCF)". These institutions are financially supported by "Nederlandse Organisatie voor Wetenschappelijk Onderzoek (NWO)".

\section{Bibliography}

[1] See the special issue Toward a Hydrogen Economy, by R. Coontz and B. Hanson, Science 305, 957 (2004).

[2] L. Schlapbach and A. Züttel, Nature 414, 353 (2001).

[3] A. Züttel, Naturwiss. Unterr. Chem. 91, 157 (2004).

[4] J. F. Stampfer Jr., C. Holley Jr., and J. F. Suttle, J. Am. Chem. Soc. 82, 3504 (1960).

[5] K. H. J. Buschow, P. C. P. Bouten, and A. R. Miedema, Rep. Prog. Phys. 45, 937 (1982).

[6] R. A. H. Niessen and P. H. L. Notten, Electrochem. Solid-State Lett. 8, A534 (2005).

[7] R. A. H. Niessen and P. H. L. Notten, J. Alloys Compd. 404, 457 (2005).

[8] P. Vermeulen, R. A. H. Niessen, and P. H. L. Notten, Electrochem. Commun. 8, 27 (2006).

[9] D. Borsa et al., Phys. Rev. B 75, 205408 (2007).

[10] S. Er, D. Tiwari, G. A. de Wijs, and G. Brocks, Phys. Rev. B 79, 024105 (2009).

[11] T. Mitchell, S. Diplas, P. Tsakiropoulos, and J. F. Watts, Philos. Mag. A 82, 841 (2002).

[12] G. Liang and R. Schulz, J. Mater. Sci. 38, 1179 (2003).

[13] S. Rousselot, M. P. Bichat, D. Guay, and L. Roue, J. Power Sources 175, 621 (2008). 
[14] D. M. Borsa et al., Appl. Phys. Lett. 88, 241910 (2006).

[15] P. Vermeulen et al., Electrochem. Solid-State Lett. 9, A520 (2006).

[16] M. Slaman et al., Sens. Actuators: B 123, 538 (2007).

[17] P. Vermeulen, E. F. M. J. van Thiel, and P. H. L. Notten, Chem. Eur. J. 13, 9892 (2007).

[18] J. P. Perdew and Y. Wang, Phys. Rev. B 45, 13244 (1992).

[19] P. E. Blöchl, Phys. Rev. B 50, 17953 (1994).

[20] G. Kresse and D. Joubert, Phys. Rev. B 59, 1758 (1999).

[21] G. Kresse and J. Hafner, Phys. Rev. B 47, 558 (1993).

[22] G. Kresse and J. Furthmüller, Phys. Rev. B 54, 11169 (1996).

[23] G. Kresse and J. Furthmüller, Comput. Mater. Sci. 6, 15 (1996).

[24] M. Methfessel and A. T. Paxton, Phys. Rev. B 40, 3616 (1989).

[25] P. E. Blöchl, O. Jepsen, and O. K. Andersen, Phys. Rev. B 49, 16223 (1994).

[26] S. Er, M. J. van Setten, G. A. de Wijs, and G. Brocks, submitted (2009).

[27] A. Zaluska, L. Zaluski, and J. O. Ström-Olsen, Appl. Phys. A: Mater. Sci. Process. 72, 157 (2001).

[28] A. A. Nayeb-Hashemi and J. B. Clark, J. Phase Equilib. Diffus. 5, 584 (1984).

[29] J. J. Vajo, F. Mertens, C. C. Ahn, R. C. B. Jr, and B. Fultz, J. Phys. Chem. B 108, 13977 (2004).

[30] M. Ekman and V. Ozolinsš, Phys. Rev. B 57, 4419 (1998).

[31] I. Ohnuma et al., Acta Mater. 48, 3113 (2000).

[32] K. Momma and F. Izumi, IUCr Newslett. 7, 106 (2006).

[33] A. Zunger, S. H. Wei, L. G. Ferreira, and J. E. Bernard, Phys. Rev. Lett. 65, 353 (1990).

[34] C. Wolverton, V. Ozolins, and A. Zunger, J. Phys.: Condens. Matter 12, 2740 (1999). 
[35] A. V. Ruban, S. I. Simak, S. Shallcross, and H. L. Skriver, Phys. Rev. B 67, 214302 (2003).

[36] H. H. van Mal, K. H. C. Buschow, and A. R. Miedema, J. Less-Common Met. 35, 65 (1974). 



\section{Part - B}

Molecular Hydrogen Storage 



\section{Chapter 6}

\section{Hydrogen storage by polylithiated molecules and nanostructures}

\subsection{Abstract}

We study polylithiated molecules as building blocks for hydrogen storage materials, using first-principles calculations. $\mathrm{CLi}_{4}$ and $\mathrm{OLi}_{2}$ bind 12 and 10 hydrogen molecules, respectively, with an average binding energy of 0.10 and $0.13 \mathrm{eV}$, leading to gravimetric densities of 37.8 and 40.3 weight $\% \mathrm{H}_{2}$. Bonding between $\mathrm{Li}$ and $\mathrm{C}$ or $\mathrm{O}$ is strongly polar and $\mathrm{H}_{2}$ molecules attach to the partially charged Li atoms without dissociating, which is favorable for (de)hydrogenation kinetics. $\mathrm{CLi}_{n}$ and $\mathrm{OLi}_{m}$ molecules can be chemically bonded to graphene sheets to hinder the aggregation of such molecules. In particular B or Be doped graphene strongly bind the molecules without seriously affecting the hydrogen binding energy. It still leads to a hydrogen storage capacity in the range $5-8.5 \mathrm{wt} . \% \mathrm{H}_{2}$.

\subsection{Introduction}

In recent years we observe a widespread effort aimed at finding alternatives to fossil fuels. The goal is to apply renewable resources that meet the increasing global demand for energy, while at the same time cut down $\mathrm{CO}_{2}$ emissions [1] Hydrogen is 
a potential energy carrier, since it can be produced sustainably and is one of the most abundant elements 2. Burning hydrogen does not affect the environment as only water is produced. On board utilization of hydrogen on a large scale is hampered, however, by the lack of efficient hydrogen storage systems. Some of the methods suggested so far are high pressure gas tanks, low temperature liquid hydrogen tanks, chemical solid state storage such as metal-hydrides, and physisorption of hydrogen by materials with a high surface area 3. Physisorption offers the possibility of storing hydrogen in molecular form. From kinetic considerations this is advantageous over chemical storage in atomic form, which requires dissociation of the hydrogen bond and the formation of a hydride.

Materials that are mainly based upon carbon are cheap, lightweight, and can have large internal surfaces. The bottleneck of physisorption by such materials lies in the weak interaction of hydrogen molecules with the host materials [4. From thermodynamic principles it can be shown that, for storage of hydrogen in physisorbed form at 30 bar under ambient temperature, and delivery at 1.5 bar, the optimum adsorption enthalpy of the hydrogen molecules is $0.15 \mathrm{eV} / \mathrm{H}_{2}$ [5]. As the adsorption enthalpy of hydrogen on activated carbon is only $\sim 0.05 \mathrm{eV} / \mathrm{H}_{2}[6$, very low temperatures or high pressures are necessary to attain meaningful storage capacities in carbon materials. One can imagine encapsulating hydrogen in carbon nanocages, but the applicability of such systems is unknown at present [7]. At the moment none of the above methods have achieved all requirements of a hydrogen storage system.

Previous studies have shown that isolated metal atoms or ions can bind hydrogen molecules, where the type of bonding depends on the metallic species. Transition metal atoms bind hydrogen molecules through weak multicenter chemical bonds called Kubas bonds [8 10]. Alkali and alkaline earth ions bind hydrogen molecules through electrostatic (charge-multipole) and polarization (charge-induced multipole) interactions [1]. Of particular interest is the lightweight $\mathrm{Li}^{+}$ion, which can bind up to six hydrogen molecules with an average binding energy of $0.19 \mathrm{eV} / \mathrm{H}_{2}$ [12. This is close to the optimal binding energy of $0.15 \mathrm{eV} / \mathrm{H}_{2}$ [5]. For this type of bonding it is essential that $\mathrm{Li}$ is ionized. Moreover, Li atoms need to be prevented from clustering, as, for instance, steric hindrance would result in a significant reduction of the amount of hydrogen molecules that can be bonded. Recent research therefore focuses on the hydrogen storage properties of Li-doped carbon nanostructures [12 27. A limited amount of Li can be introduced by doping without the Li atoms clustering, consequently restricting the wt.\% of adsorbed hydrogen significantly.

Polylithiated molecules are species that contain a large density of Li atoms. Such molecules have been studied experimentally and theoretically for a number of years, 
but never with an eye on their potential for hydrogen storage. The main focus has been on the interaction of single atoms such as $\mathrm{C}$ or $\mathrm{O}$, with multiple $\mathrm{Li}$ atoms. The first polylithiated molecule, $\mathrm{OLi}_{3}$, has been identified by mass spectroscopy by $\mathrm{Wu}$ et al. 28, and its bonding characterized quantum chemically by Schleyer et al. 29]. The latter group has also predicted the existence of the carbon based polylithiated molecules $\mathrm{CLi}_{5}$ and $\mathrm{CLi}_{6}$ 30, which has later been confirmed experimentally by Kudo 31. These studies have stimulated extensive research into the properties of $\mathrm{CLi}_{n}$ and $\mathrm{OLi}_{m}$ molecules $32[38 . \mathrm{C}-\mathrm{Li}$ and $\mathrm{O}-\mathrm{Li}$ bonds are strongly polar, resulting in a significant positive charge on the $\mathrm{Li}$ atoms. This should facilitate electrostatic bonding to hydrogen molecules, as discussed above.

In this paper we study hydrogen storage properties of the polylithiated molecules $\mathrm{CLi}_{n}, n=3-5$ and $\mathrm{OLi}_{m}, m=1-4$. We find that each Li atom can bind multiple hydrogen molecules and that the binding energy increases with the partial charge on the $\mathrm{Li}$ atom. In particular, a $\mathrm{CLi}_{4}$ molecule binds up to 12 and an $\mathrm{OLi}_{2}$ molecule up to 10 hydrogen molecules, with binding energies in the range of 0.10-0.14 and 0.13-0.21 $\mathrm{eV} / \mathrm{H}_{2}$, respectively. The corresponding gravimetric storage densities are $37.8 \mathrm{wt} . \%$ $\mathrm{H}_{2}$ for $\mathrm{CLi}_{4} \mathrm{H}_{24}$ and 40.3 wt. $\% \mathrm{H}_{2}$ for $\mathrm{OLi}_{2} \mathrm{H}_{20}$. Such extremely high densities make polylithiated molecules fascinating building blocks for hydrogen storage materials. Aggregation of $\mathrm{CLi}_{n}$ or $\mathrm{OLi}_{m}$ molecules would be detrimental for the reversibility of hydrogenation. Therefore we explore the possibilities of attaching polylithiated molecules to carbon nanostructures, in particular graphene, in order to immobilize them. Using dopants such as B or Be, one can increase the binding of the molecules to the substrate, without weakening the binding of hydrogen to the molecules. This scenario leads to several interesting structures with gravimetric densities $>5$ wt.\% $\mathrm{H}_{2}$.

\subsection{Computational Details}

The calculations are performed at the level of density functional theory (DFT), using the PW91 generalized gradient approximation (GGA) 39], and the projector augmented wave (PAW) method 40, 41, as implemented in the Vienna ab initio simulation package (VASP) [42, 43. The PAW potentials treat the $\mathrm{H} 1 s, \mathrm{Li}$ and $\mathrm{Be}$ $1 s, 2 s, \mathrm{~B}, \mathrm{C}$ and $\mathrm{O} 2 s, 2 p$ as valence electrons. With a plane wave kinetic energy cutoff of $518 \mathrm{eV}$ the total energies are converged on a scale of $10^{-3} \mathrm{eV} /$ atom. Geometries are optimized without any symmetry constraints, using the conjugate gradient (CG) algorithm. Convergence criteria of $10^{-4} \mathrm{eV}$ and $0.02 \mathrm{eV} / \AA$ are applied for the energy difference between consecutive electronic steps and the total remaining forces on the 
atoms, respectively.

Single molecules are treated within a cubic box with a cell parameter of $20 \AA$, using periodic boundary conditions and $\Gamma$-point sampling. In the graphene systems to be discussed below we use a $4 \times 4$ in-plane graphene supercell and a $3 \times 3$ regular k-point mesh to sample the Brillouin Zone (BZ). The final total energies and charge densities of the optimized structures are obtained using the corrected linear tetrahedron method 44]. The optimized geometries of a number of structures discussed in this paper are given in the supporting information. The structures are visualized using the VESTA program. The same program is used to generate the files in the supporting information [45].

The PW91 functional in combination with PAW potentials have been used previously to study the hydrogen binding properties of $\mathrm{Li}$ coated $\mathrm{C}_{60}, \mathrm{~B}_{12} \mathrm{C}_{48}$ and $\mathrm{B}_{80}$ fullerenes [20, 26, 27. To have an indication of the error bar on the total energies of the structures studied in this paper, obtained with the PW91 GGA functional, we repeat the calculations using the PBE GGA functional 46, 47. Generally, the results we obtain with these two functionals are very similar. The binding energies of $\mathrm{H}_{2}$ molecules to polylithiated molecules and nanostructures obtained with the PBE functional are $\sim 20 \mathrm{meV} / \mathrm{H}_{2}$ lower than those obtained with the PW91 functional, whereas dimerization energies of polylithiated molecules obtained with PBE are 20$60 \mathrm{meV} /$ monomer lower. As these differences are small and uniform, we only give the PW91 results in the following.

\subsection{Results and Discussion}

\subsubsection{Polylithiated Molecules}

First we analyze the interaction of molecular hydrogen with $\mathrm{CLi}_{n}$ and $\mathrm{OLi}_{m}$ molecules. As the electron affinities of $\mathrm{C}$ and $\mathrm{O}$ are much larger than that of $\mathrm{Li}$, the $\mathrm{C}-\mathrm{Li}$ and $\mathrm{O}-\mathrm{Li}$ bonds are very polar. If $\mathrm{C}$ and $\mathrm{O}$ become hypercoordinated by Li atoms, i.e. $\mathrm{CLi}_{n}$ with $n \geq 5$, or $\mathrm{OLi}_{m}$ with $m \geq 3$, the Li coordination shells become increasingly metallic and the average charge on the Li atoms decreases. The binding of hydrogen to these atoms is dominated by electrostatic and polarization interactions. Therefore, to maximize hydrogen binding energies, the charge on the $\mathrm{Li}$ atoms should be maximal and one needs to avoid hypercoordination of the $\mathrm{C}$ or $\mathrm{O}$ atoms. In $\mathrm{CLi}_{4}$ or $\mathrm{OLi}_{2}$ all valence electrons are involved in $\mathrm{C}-\mathrm{Li}$ and $\mathrm{O}-\mathrm{Li}$ bonds, respectively, maximizing the charge on the Li atoms. The most stable geometries of $\mathrm{CLi}_{4}$ and $\mathrm{OLi}_{2}$ have a high symmetry, i.e. $T_{d}$ and $D_{\infty h}$, respectively. All Li atoms 
(a)

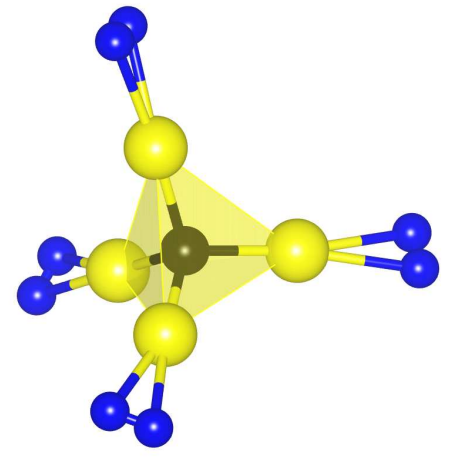

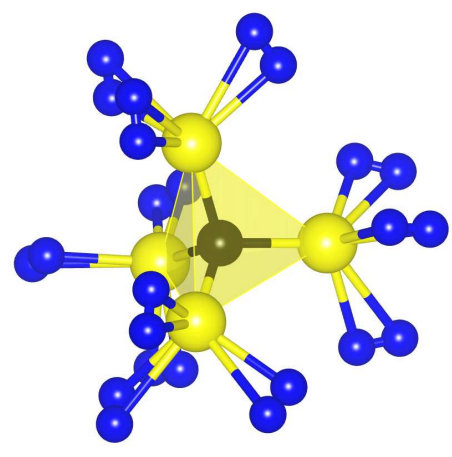

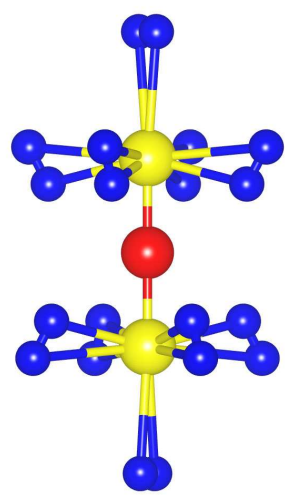

(b)

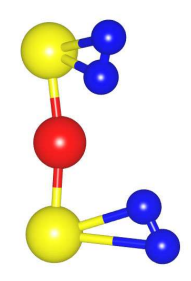

Figure 6.1: Optimized geometries of (a) $\mathrm{CLi}_{4} \mathrm{H}_{8}, \mathrm{CLi}_{4} \mathrm{H}_{24}$, and (b) $\mathrm{OLi}_{2} \mathrm{H}_{4}$, $\mathrm{OLi}_{4} \mathrm{H}_{20}$ complexes. Carbon, oxygen, lithium and hydrogen atoms are shown as black, red, yellow and blue spheres, respectively.

in a molecule are then equivalent.

A Bader charge analysis of the calculated self-consistent charge densities gives a charge of $+0.82 e$ on each of the $\mathrm{Li}$ atoms in $\mathrm{CLi}_{4}$, and a charge of $+0.88 e$ on the $\mathrm{Li}$ atoms in $\mathrm{OLi}_{2}$ [48. These high charges facilitate the electrostatic bonding of hydrogen molecules to the $\mathrm{Li}$ atoms in these molecules, similar to that in isolated $\mathrm{Li}^{+}$ions [12. Hydrogen molecules then cluster around the $\mathrm{Li}$ sites of the $\mathrm{CLi}_{4}$ and $\mathrm{OLi}_{2}$ molecules. Examples of the structures of hydrogenated molecules are shown in 6.1 and bond lengths before and after hydrogenation are given in 6.1 .

Also given in 6.1 are the hydrogen binding energies, which are calculated by subtracting the total energy of the hydrogenated polylithiated molecules from the sum of the total energies of the isolated polylithiated molecules and the hydrogen molecules, divided by the number of hydrogen molecules. Each $\mathrm{Li}$ atom in a $\mathrm{CLi}_{4}$ molecule can bind three $\mathrm{H}_{2}$ molecules as shown in 6.1 , which leads to 37.8 wt.\% $\mathrm{H}_{2}$. The average 
Table 6.1: Calculated bond lengths $(\AA)$, Bader charges $(e)$, hydrogen binding energies $E_{b}^{\mathrm{H}_{2}}\left(\mathrm{eV} / \mathrm{H}_{2}\right)$ and gravimetric hydrogen densities of $\mathrm{CLi}_{4}$ and $\mathrm{OLi}_{2}$ molecules. For $\mathrm{Li}-\mathrm{H}_{2}$ the bonding distance is measured between $\mathrm{Li}$ atom and the center of $\mathrm{H}-\mathrm{H}$ bonds. The average numbers are given and the numbers in parentheses indicate the spreads.

\begin{tabular}{lcccccc}
\hline \hline & \multicolumn{3}{c}{ bond lengths $(\AA)$} & & & \\
\cline { 2 - 4 } Molecule & $\mathrm{C}, \mathrm{O}-\mathrm{Li}$ & $\mathrm{Li}-\mathrm{H}_{2}$ & $\mathrm{H}-\mathrm{H}$ & charge on Li & $E_{b}^{\mathrm{H}_{2}}$ & wt. \% $\mathrm{H}_{2}$ \\
\hline $\mathrm{CLi}_{4}$ & $1.898(3)$ & - & - & $+0.819(2)$ & - & - \\
$\mathrm{CLi}_{4} \mathrm{H}_{8}$ & $1.888(5)$ & $2.036(12)$ & $0.756(0)$ & $+0.827(2)$ & 0.14 & 16.86 \\
$\mathrm{CLi}_{4} \mathrm{H}_{24}$ & $1.908(3)$ & $2.158(7)$ & $0.763(2)$ & $+0.851(3)$ & 0.10 & 37.82 \\
$\mathrm{OLi}_{2}$ & $1.643(0)$ & - & - & $+0.876(0)$ & - & - \\
$\mathrm{OLi}_{2} \mathrm{H}_{4}$ & $1.671(1)$ & $1.862(5)$ & $0.793(0)$ & $+0.869(0)$ & 0.21 & 11.89 \\
$\mathrm{OLi}_{2} \mathrm{H}_{20}$ & $1.711(1)$ & $2.140(144)$ & $0.764(13)$ & $+0.881(1)$ & 0.13 & 40.29 \\
\hline \hline
\end{tabular}

binding energy decreases from $0.14 \mathrm{eV}$ for the first $\mathrm{H}_{2}$ molecule (per Li atom), to 0.10 $\mathrm{eV}$ for the fully hydrogenated state. The decrease reflects a net repulsive interaction between the $\mathrm{H}_{2}$ molecules surrounding a Li atom. Steric hindrance prevents binding a fourth $\mathrm{H}_{2}$ (at least with a binding energy $>0.04 \mathrm{eV}$ ). The $\mathrm{H}_{2}$ binding energies are consistent with those found for isolated $\mathrm{Li}^{+}$ions, bearing in mind that the $\mathrm{Li}$ atoms in $\mathrm{CLi}_{4}$ have a somewhat reduced charge [11.

$\mathrm{OLi}_{2}$ molecules bind hydrogen more strongly than $\mathrm{CLi}_{4}$ molecules. As the net charge on a $\mathrm{Li}$ atom in $\mathrm{OLi}_{2}$ is larger than in $\mathrm{CLi}_{4}$ this can be expected if the binding is electrostatic. Because the $\mathrm{Li}$ atoms in $\mathrm{OLi}_{2}$ are less crowded than in $\mathrm{CLi}_{4}$, each Li atom can accommodate up to five $\mathrm{H}_{2}$ molecules, before steric hindrance prevents binding of additional hydrogen. The average binding energy decreases from $0.21 \mathrm{eV}$ for the first $\mathrm{H}_{2}$ molecule (per $\mathrm{Li}$ atom) to $0.13 \mathrm{eV}$ in the fully hydrogenated state. It leads to 40.3 wt. $\% \mathrm{H}_{2}$ for the $\mathrm{OLi}_{2} \mathrm{H}_{20}$ complex.

The fully hydrogenated $\mathrm{CLi}_{4} \mathrm{H}_{24}$ and $\mathrm{OLi}_{2} \mathrm{H}_{20}$ complexes keep a high symmetry, i.e. $T_{d}$ and $D_{2 d}$, respectively. In intermediate hydrogenation stages the symmetry can be broken, see 6.1. After hydrogenation the $\mathrm{C}-\mathrm{Li}$ and $\mathrm{O}-\mathrm{Li}$ bonds are stretched by $1 \%$ respectively $4 \%$. This is accompanied by a slight increase of the partial charge on Li by $4 \%$ respectively $0.6 \%$. The optimized calculated bond length of an isolated $\mathrm{H}_{2}$ molecule is $0.748 \AA$. 6.1 shows that in the complexes the $\mathrm{H}-\mathrm{H}$ bond lengths increase by $1-6 \%$. The limited size of these changes agrees with the notion of a weak interaction with the $\mathrm{H}_{2}$ molecules. The absolute size of these changes generally correlates with the binding energy. 
(a)

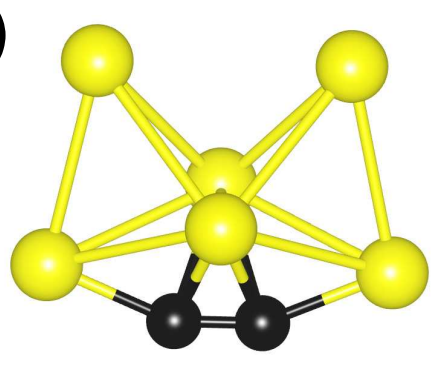

(c)

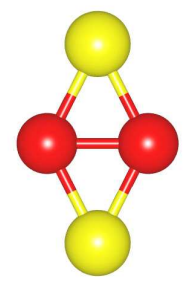

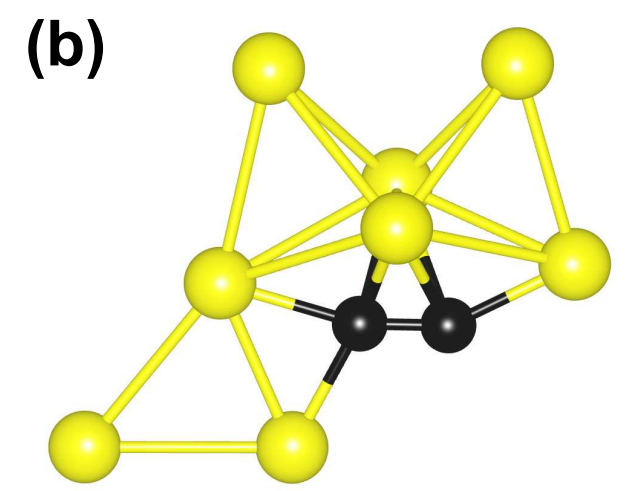

(d)

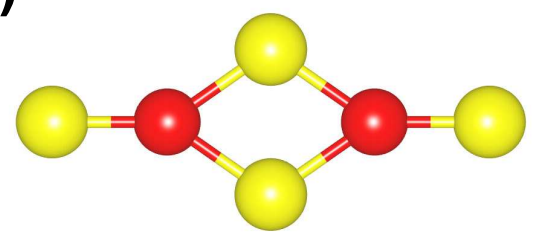

Figure 6.2: Optimized geometries of (a) $\left(\mathrm{CLi}_{3}\right)_{2}$, (b) $\left(\mathrm{CLi}_{4}\right)_{2}$, (c) $(\mathrm{OLi})_{2}$ and (d) $\left(\mathrm{OLi}_{2}\right)_{2}$.

\subsubsection{Adsorption on Graphene}

It is clear that individual $\mathrm{CLi}_{4}$ and $\mathrm{OLi}_{2}$ molecules can bind a substantial number of $\mathrm{H}_{2}$ molecules. In order to maintain reversibility during repeated hydrogen loading and unloading cycles, one should however prevent the formation of clusters or aggregates of $\mathrm{CLi}_{4}$ or $\mathrm{OLi}_{2}$ molecules. From a thermodynamic point of view such clusters could form. For instance, the equilibrium geometry of $\mathrm{C}_{2} \mathrm{Li}_{8}$ is shown in 6.2(b) [49]. The energy of this structure is $2.54 \mathrm{eV} / \mathrm{CLi}_{4}$ lower in energy than that of two separate $\mathrm{CLi}_{4}$ molecules. Similarly, two $\mathrm{OLi}_{2}$ molecules can form a cluster with a binding energy of $1.37 \mathrm{eV} / \mathrm{OLi}_{2}$, see $6.2(\mathrm{~d})$. Presumably also structures of larger clusters exist that have a favorable binding energy. One could rely on kinetic barriers preventing clustering of polylithiated molecules. Here we consider alternative possibilities, which consist of immobilizing the molecules by binding them to a holder template. Microporous carbon and carbon nanostructures would be obvious substrates for these molecules. 

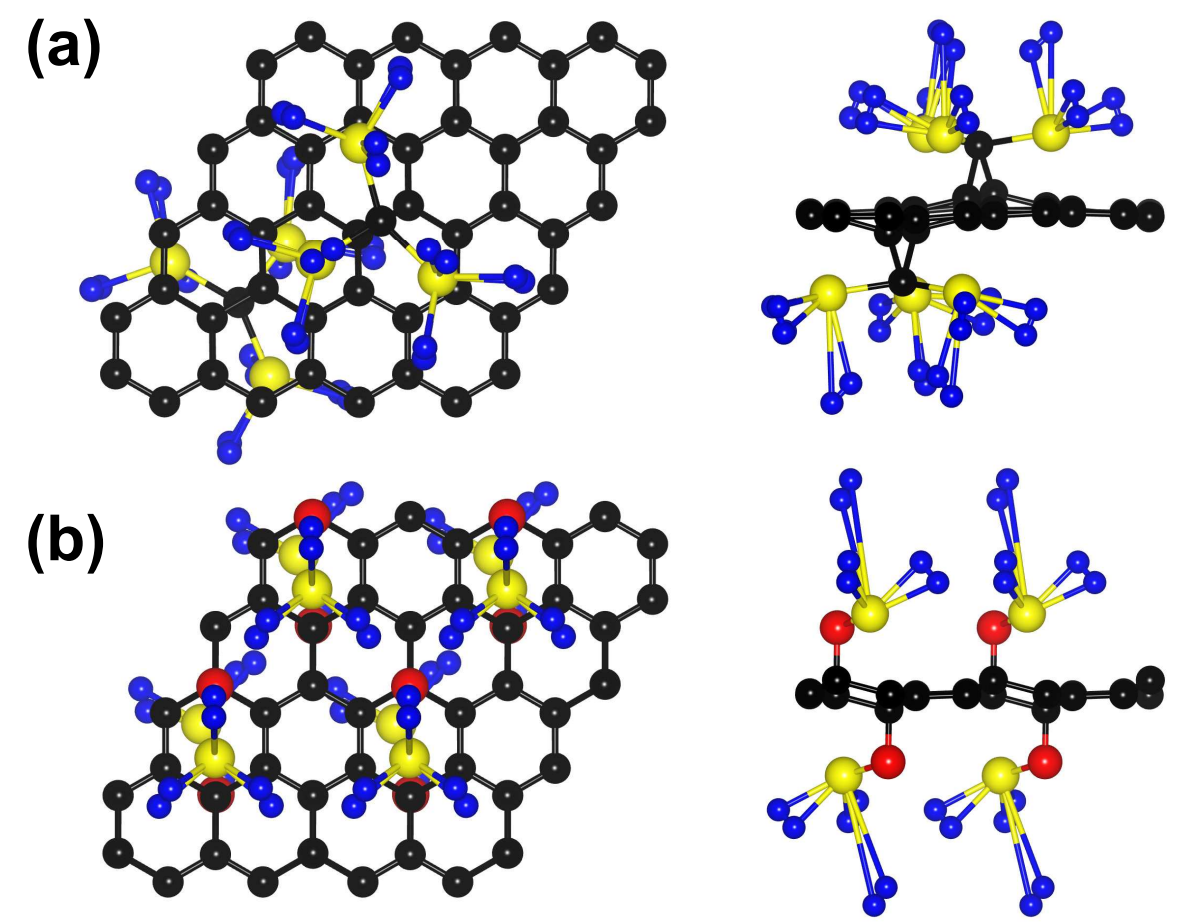

Figure 6.3: Top and side views of the optimized geometries of graphene binding (a) two $\mathrm{CLi}_{3}$ molecules, or (b) four OLi molecules per supercell, in their fully hydrogenated forms.

As a prototype system we consider binding to a single sheet of graphite, i.e. to graphene. We use a $4 \times 4$ graphene supercell, which contains 32 atoms and has an optimized cell parameter of $9.866 \AA$. This cell is sufficiently large to prevent a direct interaction between molecules bonded to graphene in neighboring cells. The calculated binding energies of single $\mathrm{CLi}_{4}$ and $\mathrm{OLi}_{2}$ molecules bonded to graphene are 0.73 and $0.55 \mathrm{eV}$, respectively. Both of these energies are considerably smaller than the corresponding molecular dimerization energies, indicating that the interaction with the substrate is not sufficiently strong. One has to consider increasing this interaction.

For this we pursue two different strategies consisting of modifying the molecules or the substrate, respectively. Removing one $\mathrm{Li}$ atom from $\mathrm{CLi}_{4}$ or $\mathrm{OLi}_{2}$ strengthens the bonding to graphene. The calculated binding energy between $\mathrm{CLi}_{3}$ and graphene is $2.00 \mathrm{eV} . \mathrm{CLi}_{3}$ molecules on graphene are preferably positioned over the center of 

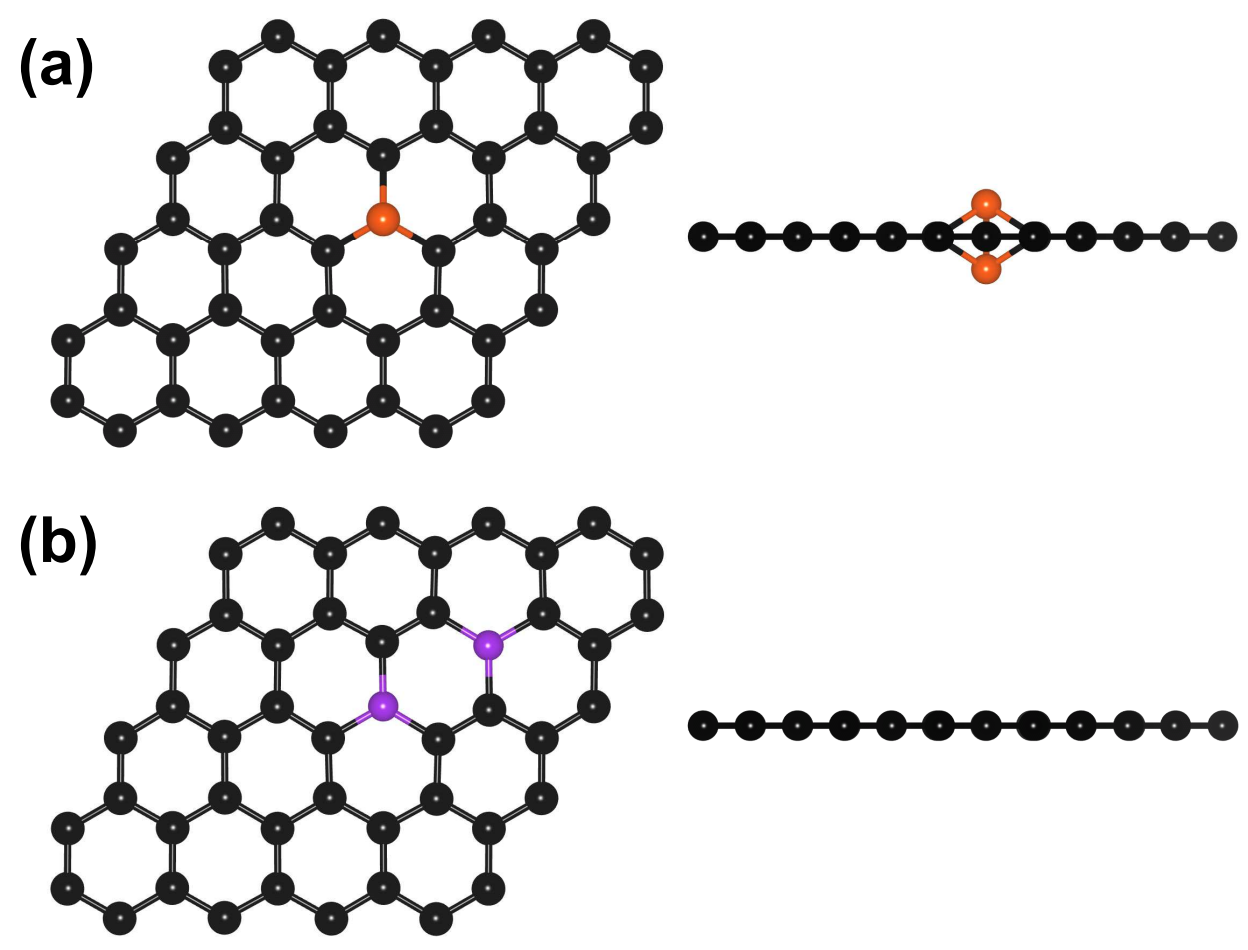

Figure 6.4: Top and side views of the optimized geometries of the (a) Be doped and (b) B doped graphene structures.

$\mathrm{C}-\mathrm{C}$ bonds. Both faces of graphene can be functionalized, which leads to a geometry as shown in 6.3 (a). This structure is stable, but it is not the absolute minimum energy structure. $\mathrm{C}_{2} \mathrm{Li}_{6}$ in its equilibrium structure, cf. 6.2(a) [50, has a binding energy of $3.86 \mathrm{eV} / \mathrm{CLi}_{3}$ with respect to two separate $\mathrm{CLi}_{3}$ molecules. It means that, although the $\mathrm{CLi}_{3}$-graphene structure is kinetically stable, there is still a thermodynamic driving force towards aggregation of $\mathrm{CLi}_{3}$ molecules.

OLi molecules also bind strongly to a graphene substrate. Since an OLi molecule is smaller than a $\mathrm{CLi}_{3}$ molecule, it is possible to quadruple the packing density of these molecules, as is shown in $6.3(\mathrm{~b})$. OLi molecules preferentially bind to the substrate through the formation of an $\mathrm{O}-\mathrm{C}$ bond. The calculated binding energy is $2.22 \mathrm{eV}$, which is comparable to the $2.26 \mathrm{eV} / \mathrm{OLi}$ aggregation energy of two OLi molecules into $\mathrm{O}_{2} \mathrm{Li}_{2}$. The $\mathrm{O}_{2} \mathrm{Li}_{2}$ molecule has a planar structure as shown in 6.2 (c). This means that the OLi-graphene structure is close to being thermodynamically stable. 

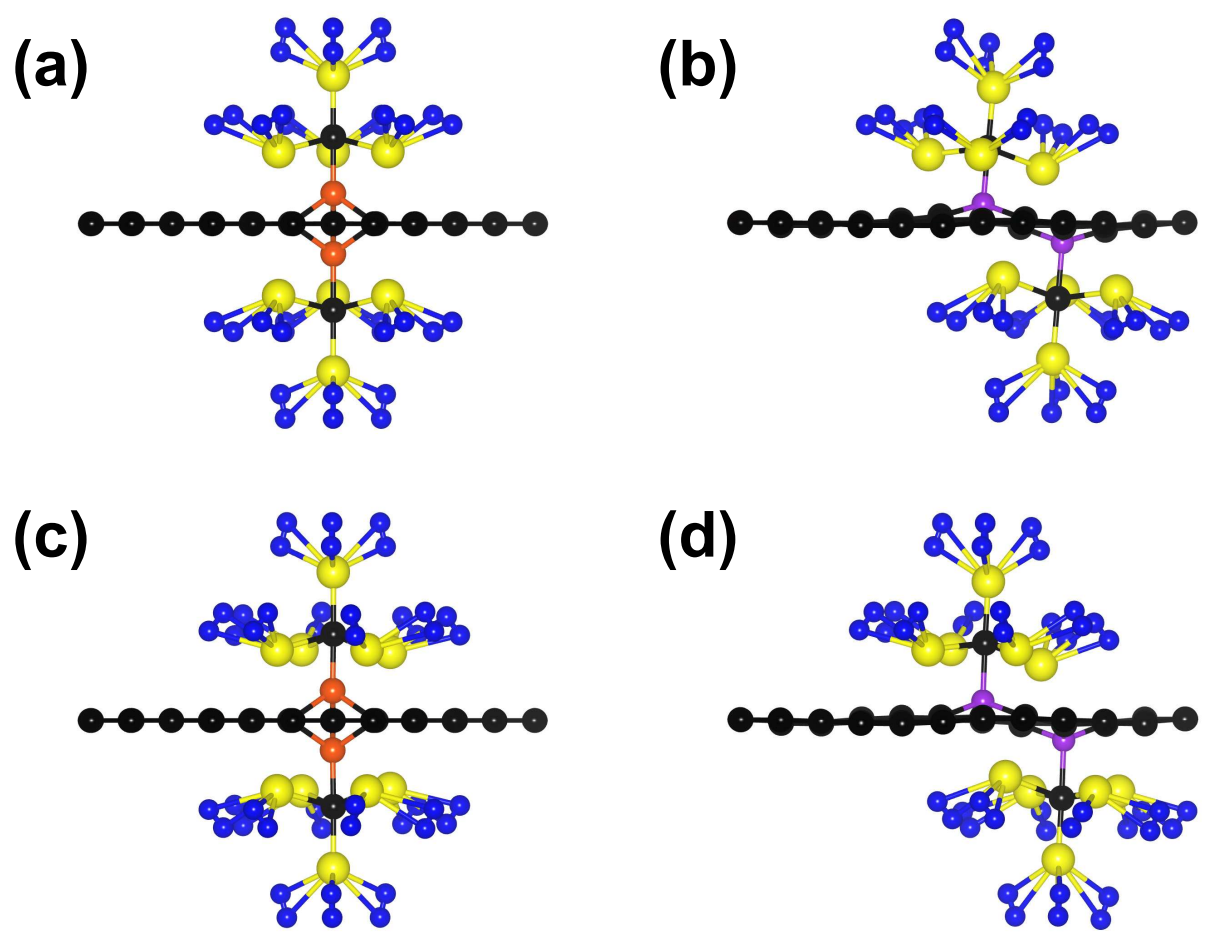

Figure 6.5: Side views of the fully hydrogenated structures of $\mathrm{CLi}_{4}$ on (a) Be doped and (b) B doped graphene and of $\mathrm{CLi}_{5}$ on (c) Be doped and (d) B doped graphene.

\subsubsection{Adsorption on Doped Graphene}

Another possible way of increasing the binding between polylithiated molecules and the substrate is by chemical modification of the graphene layer [51. Introducing an impurity in graphene might for instance not only strengthen the interaction with $\mathrm{CLi}_{n}$ and $\mathrm{OLi}_{m}$ molecules, but could also maintain the positive charge on the $\mathrm{Li}$ atoms, and therefore the binding to $\mathrm{H}_{2}$ molecules. Here we consider modification of graphene by atomic substitution of $\mathrm{C}$ by $\mathrm{B}$ or Be atoms. Previous studies have shown that $\mathrm{B}$ doping of graphene does not cause any major deformation of the graphene structure [52]. B doping concentrations of as high as 17 at. \% can be achieved in thin films [53. From experiment it has been suggested that the preferential geometry upon high $\mathrm{B}$ doping has two $\mathrm{B}$ atoms in para positions of an hexagonal ring in the graphene plane, as shown in 6.4(b). Indeed, the stability of this geometry has recently been confirmed by a theoretical study [54. Here we consider such a double B substitution 
Table 6.2: Binding energies $(\mathrm{eV})$ between $\mathrm{CLi}_{n}$ and $\mathrm{OLi}_{m}$ molecules and (doped) graphene, and dimerization energies $\left(\mathrm{CLi}_{n}\right)_{2},\left(\mathrm{OLi}_{m}\right)_{2}$.

\begin{tabular}{lrrrrrrr}
\hline \hline & $\mathrm{CLi}_{3}$ & $\mathrm{CLi}_{4}$ & $\mathrm{CLi}_{5}$ & $\mathrm{OLi}$ & $\mathrm{OLi}_{2}$ & $\mathrm{OLi}_{3}$ & $\mathrm{OLi}_{4}$ \\
\hline undoped & 2.00 & 0.73 & & 2.22 & 0.55 & & \\
Be doped & 4.12 & 3.81 & 3.96 & 4.14 & 3.76 & 3.55 & 3.00 \\
B doped & 4.05 & 2.91 & 3.23 & 3.45 & 2.49 & 3.13 & 3.69 \\
dimer & 3.86 & 2.54 & 1.57 & 2.26 & 1.37 & 1.17 & 0.34 \\
\hline \hline
\end{tabular}

in our $4 \times 4$ graphene supercell, which gives a doping concentration of 6.25 at. $\%$.

As an alternative to $\mathrm{B}$ doping we also consider substituting $\mathrm{C}$ with $\mathrm{Be}$ atoms. Replacing one $\mathrm{C}$ with a Be atom does not alter the planar structure of graphene. A single Be atom binds to a vacancy in the graphene lattice with a binding energy of $2.67 \mathrm{eV}$ (with respect to the bulk Be metal). It is energetically advantageous however to replace a single $\mathrm{C}$ atom by a pair of Be atoms. The second Be atom then binds with a binding energy of $0.69 \mathrm{eV}$ (again, with respect to bulk Be). The two Be atoms are then displaced above and below the graphene plane by $0.855 \AA$, as shown in 6.4(a), whereas the rest of graphene maintains its original structure. The Be atoms can act as pedestals that increase the distance between the graphene plane and the molecules we are going to attach, which could limit steric hindrance. All of the Be or B doped graphene structures have nonmagnetic ground states.

Modifying graphene by Be or B doping increases its interaction with polylithiated molecules. The molecules preferentially bind to the dopant atoms and form $\mathrm{C}, \mathrm{O}-\mathrm{B}, \mathrm{Be}$ bonds. From the point of view of binding strength, there is no need to modify the molecules by extracting a Li atom before attaching them to the substrate. $\mathrm{CLi}_{4}$ binds to a $\mathrm{B}$ or a Be site with binding energies of $2.91,3.81 \mathrm{eV}$, respectively. The binding geometries are shown in 6.5. The binding between $\mathrm{OLi}_{2}$ and $\mathrm{B}$ or $\mathrm{Be}$ doped graphene has an energy of $2.49,3.76 \mathrm{eV}$, respectively. Since all these binding energies are well above the molecular dimerization energies one expects that aggregation of these molecules is now suppressed. Remarkably, doped graphene also forms strong bonds to other $\mathrm{CLi}_{n}$ and $\mathrm{OLi}_{m}$ molecules, including hypercoordinated ones such as $\mathrm{CLi}_{5}, \mathrm{OLi}_{3}$ and $\mathrm{OLi}_{4}$, leading to similar binding geometries. The binding energies are given in 6.2 and examples of binding geometries are shown in 6.5 .

With the polylithiated molecules strongly bonded to (doped) graphene we now study the hydrogenation properties of these systems. For $\mathrm{CLi}_{3}$ and $\mathrm{OLi}$ bonded to undoped graphene we find that each of the $\mathrm{Li}$ atoms in the molecules above and 
Table 6.3: Average hydrogen binding energies $E_{b}^{\mathrm{H}_{2}}\left(\mathrm{eV} / \mathrm{H}_{2}\right)$, number of hydrogen molecules $\# \mathrm{H}_{2}$ bonded per $\mathrm{CLi}_{n}$ or $\mathrm{OLi}_{m}$ species, and gravimetric hydrogen densities of polylithiated molecules bonded to (doped) graphene.

\begin{tabular}{|c|c|c|c|c|c|c|c|c|c|}
\hline & \multicolumn{3}{|c|}{ undoped } & \multicolumn{3}{|c|}{ Be doped } & \multicolumn{3}{|c|}{ B doped } \\
\hline & $E_{b}^{\mathrm{H}_{2}}$ & $\# \mathrm{H}_{2}$ & wt. $\% \mathrm{H}_{2}$ & $E_{b}^{\mathrm{H}_{2}}$ & $\# \mathrm{H}_{2}$ & wt. $\% \mathrm{H}_{2}$ & $E_{b}^{\mathrm{H}_{2}}$ & $\# \mathrm{H}_{2}$ & wt. $\% \mathrm{H}_{2}$ \\
\hline $\mathrm{CLi}_{3}$ & 0.10 & 9 & 7.46 & 0.15 & 7 & 5.83 & 0.11 & 8 & 6.72 \\
\hline $\mathrm{CLi}_{4}$ & & & & 0.09 & 9 & 7.17 & 0.09 & 9 & 7.29 \\
\hline $\mathrm{CLi}_{5}$ & & & & 0.08 & 11 & 8.40 & 0.07 & 11 & 8.53 \\
\hline OLi & 0.12 & 3 & 7.85 & 0.23 & 3 & 2.70 & 0.10 & 3 & 2.75 \\
\hline $\mathrm{OLi}_{2}$ & & & & 0.10 & 6 & 5.10 & 0.09 & 6 & 5.19 \\
\hline $\mathrm{OLi}_{3}$ & & & & 0.12 & 6 & 4.96 & 0.10 & 6 & 5.04 \\
\hline $\mathrm{OLi}_{4}$ & & & & 0.08 & 9 & 7.06 & 0.06 & 9 & 7.18 \\
\hline
\end{tabular}

below the graphene plane can bind three $\mathrm{H}_{2}$ molecules, see 6.3 The average hydrogen binding energy is 0.10 and $0.12 \mathrm{eV}$, respectively, which is comparable to that in free $\mathrm{CLi}_{4}$ and $\mathrm{OLi}_{2}$ molecules, compare 6.1. The resulting geometries are given in 6.3 The gravimetric densities in the $\mathrm{CLi}_{3}$ - and OLi-graphene systems are 7.46 and 7.85 wt. $\% \mathrm{H}_{2}$, respectively.

As discussed above, doping of graphene with Be or B increases the binding of polylithiated molecules to the substrate. The effect of doping on the binding energy between hydrogen and these molecules is small in most cases, as can be observed in 6.3. Only for $\mathrm{H}_{2}$ on $\mathrm{CLi}_{3}$ and OLi bonded to Be doped graphene the binding energy is significantly increased. The distance between the hydrogen molecules and the graphene plane is larger in these systems, as compared to the other cases. The gravimetric hydrogen density in the doped graphene systems is determined by the concentration of dopant atoms, since the polylithiated molecules are bonded to these atoms, as well as by the number of hydrogen molecules bonded to a single polylithiated molecule.

The latter is foremost determined by steric hindrance. A Li atom that is close to the graphene plane or to other Li atoms can in general capture only one or two $\mathrm{H}_{2}$ molecules, whereas the ones that are further away from the plane and other $\mathrm{Li}$ atoms can bind three $\mathrm{H}_{2}$ molecules. This is illustrated in 6.5 We have fixed the doping concentration of graphene. In particular for the $\mathrm{OLi}_{m}$ systems one can still increase the doping concentration without introducing too much steric hindrance, which would increase the gravimetric density substantially. 
Hydrogen binding energies decrease slightly for hypercoordinated polylithiated molecules, i.e. $\mathrm{CLi}_{n}, n>3$ and $\mathrm{OLi}_{m}, m>1$. 6.5 shows that this leads to a tradeoff between hydrogen binding energy and gravimetric density. Nevertheless it is clear that there are several possible systems and structures that attain a gravimetric density higher than $5 \% \mathrm{H}_{2}$ with $\mathrm{H}_{2}$ binding energies in excess of $0.1 \mathrm{eV}$.

\subsection{Conclusions}

In conclusion, we have shown by means of first-principles DFT calculations that polylithiated molecules can be used as versatile building blocks for hydrogen storage materials. $\mathrm{Li}-\mathrm{C}$ and $\mathrm{Li}-\mathrm{O}$ bonds are strongly polar and the partially charged $\mathrm{Li}$ atoms can attract up to three to five $\mathrm{H}_{2}$ molecules with an average binding energy in the range $0.10-0.15 \mathrm{eV} . \mathrm{CLi}_{4}$ and $\mathrm{OLi}_{2}$ molecules can then bind 12 and 10 hydrogen molecules, respectively, giving very high gravimetric densities of 37.8 and 40.3 weight $\% \mathrm{H}_{2}$. Binding polylithiated molecules to carbon nanostructures such as graphene can be used to immobilize them. In particular doping graphene with $\mathrm{B}$ or Be increases the binding energy. Although there is a trade-off between the size of the $\mathrm{H}_{2}$ adsorption energy and its gravimetric density in these systems, densities in the range 5-8.5 wt.\% $\mathrm{H}_{2}$ can be reached without seriously decreasing the adsorption energy.

\section{Acknowledgments}

This work is part of the research programs of "Advanced Chemical Technologies for Sustainability (ACTS)" and the "Stichting voor Fundamenteel Onderzoek der Materie (FOM)". The use of supercomputer facilities was sponsored by the "Stichting Nationale Computerfaciliteiten (NCF)". These institutions are financially supported by "Nederlandse Organisatie voor Wetenschappelijk Onderzoek (NWO)".

\section{Supplementary Info}

Optimized structures of free-standing (hydrogenated) polylithiated molecules and of (hydrogenated) polylithiated molecules adsorbed on doped graphene are supplied in CIF (Crystallographic Information File) format. This information is available free of charge via the Internet at http://pubs.acs.org. 


\section{Bibliography}

[1] S. Pacala and R. Socolow, Science 305, 968 (2004).

[2] See the special issue Toward a Hydrogen Economy, by R. Coontz and B. Hanson, Science 305, 957 (2004).

[3] L. Schlapbach and A. Züttel, Nature 414, 353 (2001).

[4] S. Patchkovskii et al., Proc. Natl. Acad. Sci. U.S.A. 102, 10439 (2005).

[5] S. K. Bhatia and A. L. Myers, Langmuir 22, 1688 (2006).

[6] B. Panella, M. Hirscher, and S. Roth, Carbon 43, 2209 (2005).

[7] O. V. Pupysheva, A. A. Farajian, and B. I. Yakobson, Nano Lett. 8, 767 (2008).

[8] J. Niu, B. K. Rao, and P. Jena, Phys. Rev. Lett. 68, 2277 (1992).

[9] L. Gagliardi and P. Pyykkö, J. Am. Chem. Soc. 126, 15014 (2004).

[10] E. Durgun, S. Ciraci, W. Zhou, and T. Yildirim, Phys. Rev. Lett. 97, 226102 (2006).

[11] R. C. Lochan and M. Head-Gordon, Phys. Chem. Chem. Phys. 8, 1357 (2006).

[12] K. R. S. Chandrakumar and S. K. Ghosh, Nano Lett. 8, 13 (2008).

[13] P. Chen, X. Wu, J. Lin, and K. L. Tan, Science 285, 91 (1999).

[14] Z. Zhou, X. Gao, J. Yan, D. Song, and M. Morinaga, Carbon 42, 2677 (2004).

[15] W. Q. Deng, X. Xu, and W. A. Goddard, Phys. Rev. Lett. 92, 166103 (2004).

[16] O. Maresca, R. J. M. Pellenq, F. Marinelli, and J. Conard, J. Chem. Phys. 121, 12548 (2004).

[17] Z. H. Zhu, G. Q. Lu, and S. C. Smith, Carbon 42, 2509 (2004).

[18] I. Cabria, M. J. López, and J. A. Alonso, J. Chem. Phys. 123, 204721 (2005).

[19] Y. Zhang, L. G. Scanlon, M. A. Rottmayer, and P. B. Balbuena, J. Phys. Chem. B 110, 22532 (2006).

[20] Q. Sun, P. Jena, Q. Wang, and M. Marquez, J. Am. Chem. Soc. 128, 9741 (2006). 
[21] B. Fang, H. Zhou, and I. Honma, Appl. Phys. Lett. 89, 023102 (2006).

[22] J. H. Cho and C. R. Park, Catal. Today 120, 407 (2007).

[23] S. Han and W. Goddard III, J. Am. Chem. Soc. 129, 8422 (2007).

[24] A. Blomqvist, C. M. Araujo, P. Srepusharawoot, and R. Ahuja, Proc. Natl. Acad. Sci. U.S.A. 104, 20173 (2007).

[25] L. Chen, Y. Zhang, N. Koratkar, P. Jena, and S. K. Nayak, Phys. Rev. B 77, 33405 (2008).

[26] Y. Li and R. T. Yang, J. Phys. Chem. C 112, 19268 (2008).

[27] Q. Sun, Q. Wang, and P. Jena, Appl. Phys. Lett. 94, 013111 (2009).

[28] C. H. Wu, H. Kudo, and H. R. Ihle, J. Chem. Phys. 70, 1815 (1979).

[29] P. v. R. Schleyer, E. U. Wuerthwein, and J. A. Pople, J. Am. Chem. Soc. 104, 5839 (1982).

[30] P. v. R. Schleyer, E. U. Wuerthwein, E. Kaufmann, T. Clark, and J. A. Pople, J. Am. Chem. Soc. 105, 5930 (1983).

[31] H. Kudo, Nature 355, 432 (1992).

[32] P. v. R. Schleyer and J. Kapp, Chem. Phys. Lett. 255, 363 (1996).

[33] P. Lievens et al., Eur. Phys. J. D 9, 289 (1999).

[34] E. D. Jemmis et al., J. Am. Chem. Soc. 104, 4275 (1982).

[35] J. Ivanic and C. J. Marsden, J. Am. Chem. Soc. 115, 7503 (1993).

[36] H. Kudo and C. H. Wu, J. Nucl. Mater. 201, 261 (1993).

[37] R. Ponec et al., J. Phys. Chem. A 106, 1019 (2002).

[38] W. Zhizhong, Z. Xiange, and T. Auchin, J. Mol. Struct. 453, 225 (1998).

[39] J. P. Perdew et al., Phys. Rev. B 46, 6671 (1992).

[40] P. E. Blöchl, Phys. Rev. B 50, 17953 (1994).

[41] G. Kresse and D. Joubert, Phys. Rev. B 59, 1758 (1999).

[42] G. Kresse and J. Hafner, Phys. Rev. B 47, 558 (1993). 
[43] G. Kresse and J. Furthmüller, Phys. Rev. B 54, 11169 (1996).

[44] P. E. Blöchl, O. Jepsen, and O. K. Andersen, Phys. Rev. B 49, 16223 (1994).

[45] K. Momma and F. Izumi, J. Appl. Crystallogr. 41, 653 (2008).

[46] J. P. Perdew, K. Burke, and M. Ernzerhof, Phys. Rev. Lett. 77, 3865 (1996).

[47] J. P. Perdew, K. Burke, and M. Ernzerhof, Phys. Rev. Lett. 78, 1396 (1997).

[48] G. Henkelman, A. Arnaldsson, and H. Jónsson, Comput. Mater. Sci. 36, 354 (2006).

[49] J. Ivanic and C. J. Marsden, Organometallics 13, 5141 (1994).

[50] A. M. Sapse and P. v. R. Schleyer, Lithium Chemistry: A Theoretical and Experimental Overview (Wiley-IEEE, 1995).

[51] D. W. Boukhvalov and M. I. Katsnelson, Nano Lett. 8, 4373 (2008).

[52] M. Endo, T. Hayashi, S. Hong, T. Enoki, and M. S. Dresselhaus, J. Appl. Phys. 90, 5670 (2001).

[53] C. T. Hach, L. E. Jones, C. Crossland, and P. A. Thrower, Carbon 37, 221 (1999).

[54] R. H. Miwa, T. B. Martins, and A. Fazzio, Nanotechnology 19, 155708 (2008). 


\section{Chapter 7}

\section{DFT study of planar boron sheets: A new template for hydrogen storage}

\subsection{Abstract}

We study the hydrogen storage properties of planar boron sheets and compare them to those of graphene. The binding of molecular hydrogen to the boron sheet $(0.05 \mathrm{eV})$ is stronger than that to graphene. We find that dispersion of alkali metal $(\mathrm{AM}=\mathrm{Li}, \mathrm{Na}$, and $\mathrm{K})$ atoms onto the boron sheet markedly increases hydrogen binding energies and storage capacities. The unique structure of the boron sheet presents a template for creating a stable lattice of strongly bonded metal atoms with a large nearest neighbor distance. In contrast, AM atoms dispersed on graphene tend to cluster to form a bulk metal. In particular the boron-Li system is found to be a good candidate for hydrogen storage purposes. In the fully loaded case this compound can contain up to $10.7 \mathrm{wt} \%$ molecular hydrogen with an average binding energy of $0.15 \mathrm{eV} / \mathrm{H}_{2}$.

\subsection{Introduction}

Hydrogen is an abundant, clean, and renewable energy carrier [1. An important barrier preventing the large scale use of hydrogen is storing it densely and safely un- 
der moderate conditions [2]. Also, fast loading and unloading of the storage system is still a challenge. Storage of hydrogen in molecular form may be more beneficial than storage of atomic hydrogen in chemical hydrides. Complex metal hydrides, for instance, are often either too stable or too unstable [3, 4] and require substantial doping to tune their stability [5, 6]. Moreover, the formation and decomposition of chemical hydrides typically involve complicated solid-state chemical reactions, which hamper the kinetics of hydrogen loading and unloading. In contrast, materials that physisorb molecular hydrogen, such as graphene, carbon nanotubes and fullerenes, clathrates, zeolites, and metal organic frameworks (MOFs), are capable of achieving fast hydrogen kinetics. The binding energy of hydrogen to these host materials is however small, which leads to unfavorable operating conditions of very low temperatures or high hydrogen pressures [7].

Recent studies show that metal doping enhances the strength of binding between hydrogen molecules and physisorption materials [8, 9]. An ideal metal dopant should strongly bind to the host material, and it should bind hydrogen molecules effectively. For instance, for storage of molecular hydrogen at 30 bar under ambient temperature, and delivery at 1.5 bar, the optimum adsorption enthalpy needs to be $0.15 \mathrm{eV} / \mathrm{H}_{2}$ [7]. Upon deposition, the geometrical positioning of metal atoms over the substrate is also important, since the number of $\mathrm{H}_{2}$ molecules per dopant site should be maximized. To prevent steric hindrance, this usually means that the dopant atoms need to be far apart. Therefore, the material should resist the clustering of metal atoms. At least the binding energy of a metal atom to the substrate should then be larger than the cohesive energy of the bulk metal. This is extremely difficult to attain with transition metal dopants, since the cohesive energies of bulk transition metals are high. Alkali metals have much lower cohesive energies and are therefore more suitable as dopants.

Recent studies addressed the alkali metal doping of physisorption materials such as graphene [10 12, fullerene [13 15], carbon nanotubes [16-19], and MOFs [20 23]. Hydrogen molecules are trapped around the dispersed doping metal atoms through electrostatic and polarization interactions [24. In addition to increasing the binding strengths to hydrogen, alkali metal doping also increases the effective surface area available for absorption in some cases. Hydrogen adsorption then goes beyond a single monolayer, increasing the volumetric and gravimetric hydrogen densities accordingly.

In order to obtain a sufficiently high gravimetric density, not only the alkali metals need to be lightweight, but also the substrates need to be lightweight materials. In the past decade, carbon nanostructures of various dimensions have been studied extensively both theoretically and experimentally for various applications, including as substrates for hydrogen storage. The recent realization of the structurally most 


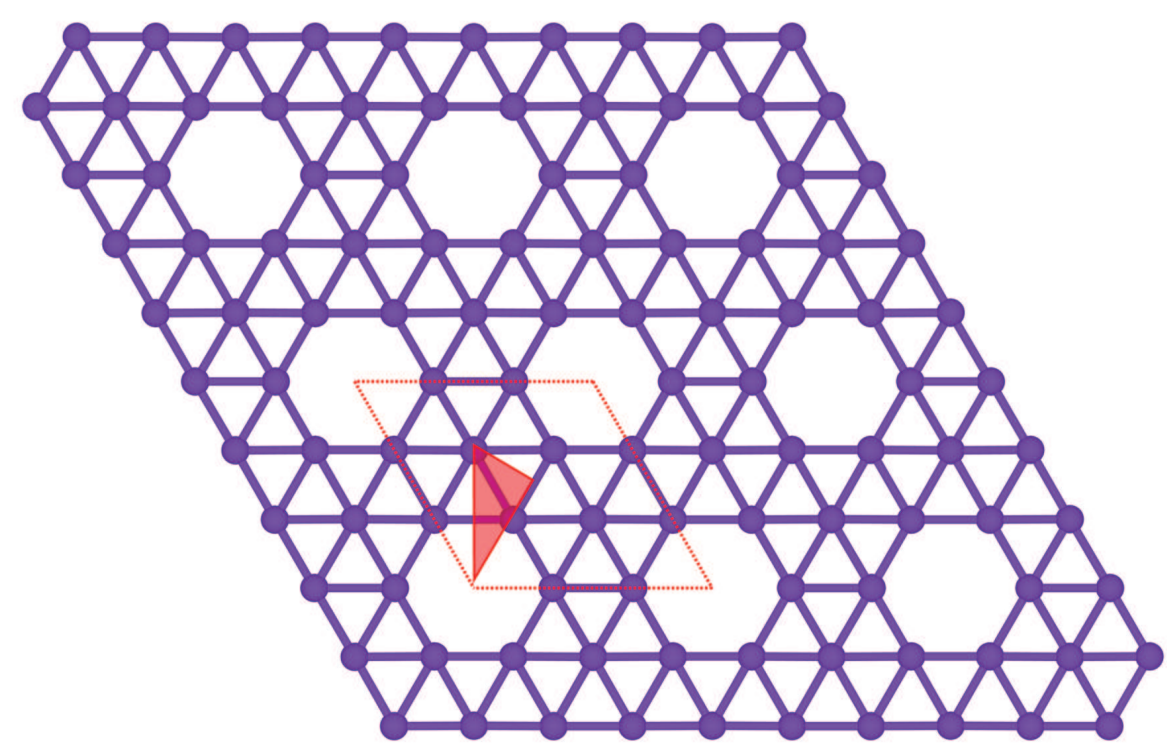

Figure 7.1: The most stable form of the boron sheet. The primitive cell is indicated by dotted red lines. It contains eight boron atoms, shown as purple spheres. The irreducible part of the Wigner-Seitz cell (WSC) is indicated by a triangle.

simple carbon network, graphene, has attracted a great deal of interest [25, 26]. The lightest element that can form extensive covalently bonded structures is in fact boron. Boron, having one electron less than carbon, forms icosahedral clusters, [27, 28] and has long been thought not to form two-dimensional (2-D) structures similar to graphene.

However, it has very recently been shown that the stability of planar 2-D hexagonal boron sheets is enhanced markedly by inserting additional boron atoms in the centers of hexagons of the honeycomb structure; see 7.1$] 2932$. These additional boron atoms modify the electronic structure of the boron lattice. 2-D hexagonal boron is an electron deficient system with part of the $\mathrm{sp}^{2}$ bonding states unoccupied. The electrons provided by the additional boron atoms occupy these states, increasing the stability 29. These new 2-D boron structures also provide insight into the unusual stability of other boron-only structures with different dimensions, such as fullerenes [33 35], nanotubes [32, 36, 37], and nanoribbons [38].

Here, we investigate the hydrogen storage properties of the boron sheet structure that is identified as the most stable form, the so-called $\alpha$-structure. [29] We first 
study the interaction of $\mathrm{H}_{2}$ molecules with this boron sheet. Next, the dispersion of alkali metal $(\mathrm{AM}=\mathrm{Li}, \mathrm{Na}$, and $\mathrm{K}$ ) atoms onto the boron sheet is considered. We study the binding and the mobility of these AM atoms on the boron sheet and the hydrogenation energies of these systems. All of the three AMs are found to bind strongly to the boron sheet. Moreover, the unique structure of the boron sheet allows for a stable lattice of AM atoms with a large nearest neighbor AM-AM distance, which consequently creates space for hydrogenation. The AM atoms accumulate a net positive charge, which not only stabilizes this structure but also strengthens the binding to hydrogen molecules. All three AMs lead to a higher hydrogen binding energy, but Li is by far the best doping element.

\subsection{Computational Details}

We perform first-principles calculations based on density functional theory (DFT), using the Vienna ab initio simulation package (VASP) [39, 40]. The generalized gradient approximation (GGA) in the form of the PW91 functional is used to approximate exchange and correlation [41. Although dispersion (van der Waals) interactions are not captured by this functional, it should give a good description of chemical bonding and of electrostatic interactions, which are the most prominent interactions in alkali doped systems; see also ref 12 . Binding energies of $\mathrm{H}_{2}$ molecules to similar systems obtained with the PW91 functional are within $\sim 20 \mathrm{meV} / \mathrm{H}_{2}$ of those obtained with the PBE functional [12]. Therefore, we only give the PW91 results in the following.

The basis set is constructed according to the projector augmented wave (PAW) method [42, 43. The following electrons are treated as valence: $\mathrm{H} 1 \mathrm{~s}, \mathrm{~B} 2 \mathrm{~s} 2 \mathrm{p}, \mathrm{Li}$ 1s2s, Na 3s2p, and K 4s3p. A cutoff energy of $400 \mathrm{eV}$ is used for the plane-wave basis. Brillouin zones (BZs) of all structures are integrated with $k$-point spacings of $\sim 0.01 \AA^{-1}$ [4]. For instance, the BZ corresponding to the unit cell shown in Figure 7.1 is then integrated using a $12 \times 12$ regular grid. Gaussian smearing is used with a smearing width of $0.01 \mathrm{eV}$. Periodic images of the sheet along the surface normal are well separated by a distance of $\sim 20 \AA$, so that the interactions between these images are negligible. Atomic and geometric relaxations are carried out by employing the conjugated-gradient (CG) algorithm. All atomic positions and cell parameters are relaxed. The relaxations are assumed to be complete when the total force remaining on each atom and the stress tensor components acting on the cells are less than $0.02 \mathrm{eV} / \AA$ and $2.5 \mathrm{kbar}$, respectively. Total energies then are converged to within 1 $\mathrm{meV} /$ unit cell at least. The tetrahedron method is then used to calculate the total energies of the relaxed structures [45]. 

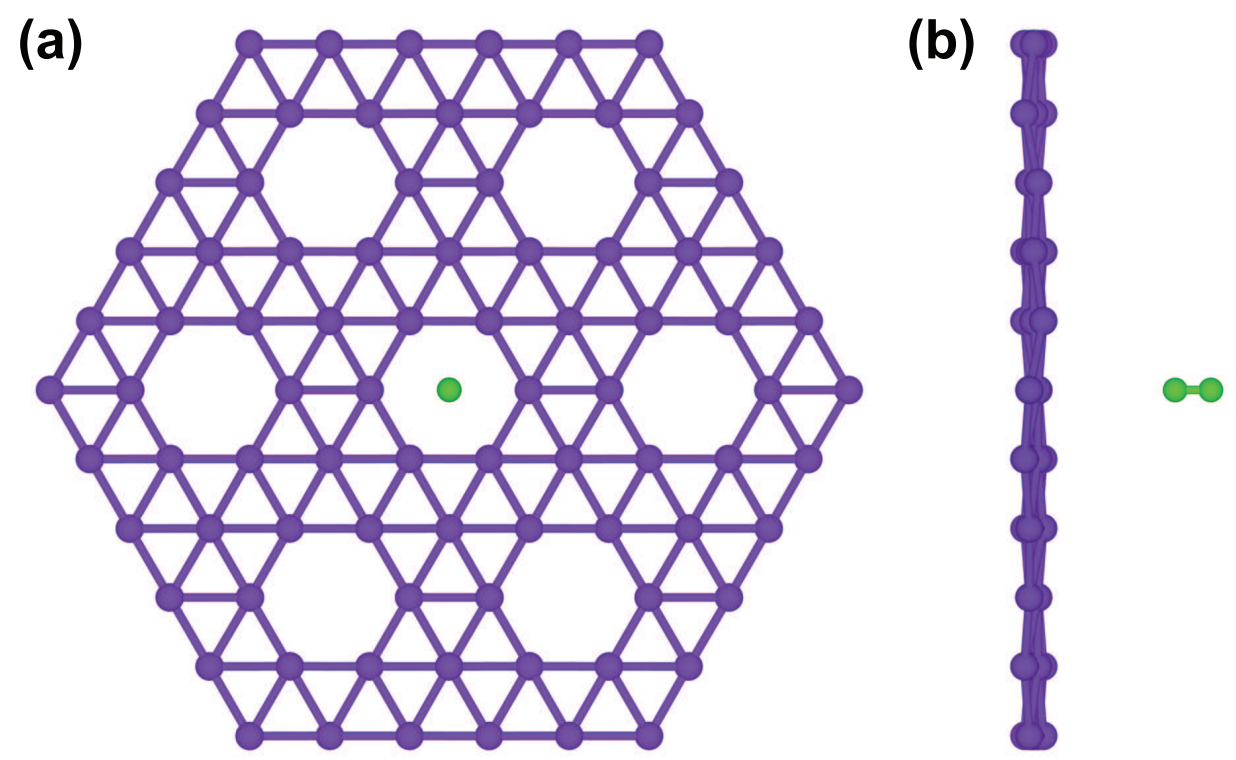

Figure 7.2: The most stable geometry of $\mathrm{H}_{2}$ adsorbed on a boron sheet shown in (a) top, and (b) side views. The molecule is positioned over the center of an empty boron hexagon with its axis orthogonal to the boron plane.

\subsection{Results and Discussion}

\subsubsection{Hydrogen Adsorption on Boron Sheets}

The most stable structure of a boron sheet is shown in 7.1. It is a graphene-like structure, where two-thirds of the hexagon centers are occupied by additional boron atoms. The primitive cell of the sheet then contains eight boron atoms. It leads to a unique planar structure with a lattice of empty hexagons spaced at a distance of $5.06 \AA$ of one another.

To model the interaction of hydrogen molecules with the boron sheet, we use a $2 \times 2$ supercell. The distance between hydrogen molecules in neighboring cells is then $10.12 \AA$, which is sufficiently large to neglect the interaction between these molecules. We consider a number of possible positions of the $\mathrm{H}_{2}$ molecule on the boron sheet. Since the latter contains a lot of symmetry, it is only necessary to consider the irreducible part of the Wigner-Seitz cell (WSC), which is indicated in 7.1. We scan the edges of the irreducible WSC by placing a $\mathrm{H}_{2}$ molecule at several positions, which are separated by $0.8 \AA$, as measured from the $\mathrm{H}-\mathrm{H}$ bond centers. 
At each of these points, we consider three different molecular orientations. In two of these orientations, the $\mathrm{H}_{2}$ molecular axis is parallel to the boron plane. The two orientations differ by an in-plane angle of $30^{\circ}$. In the third configuration, the $\mathrm{H}_{2}$ molecular axis is perpendicular to the boron plane. The $\mathrm{H}-\mathrm{H}$ bond centers are placed at a distance of at least $2 \AA$ from the boron sheet.

In total, we have constructed a set of 36 distinct initial geometries. All of the structures in this set are then subjected to relaxation without any symmetry constraints. These calculations reveal that $\mathrm{H}_{2}$ molecules tend to occupy positions over the open boron hexagons. The minimum energy configuration is the one in which the molecule is over the center of an open hexagon with its axis perpendicular to the boron plane (7.2). The distance between the boron plane and the center of the molecule is $3.27 \AA$, and the calculated value of the binding energy is $47 \mathrm{meV}$. The energy differences between different orientations of the $\mathrm{H}_{2}$ molecule are generally small. $\mathrm{A} \mathrm{H}_{2}$ molecule at this site with its axis parallel to the boron plane is only $\sim 5$ meV higher in energy. The potential energy surface obtained by varying the position of the $\mathrm{H}_{2}$ molecule over the plane has a corrugation of $61 \mathrm{meV}$.

Interestingly, the interaction of $\mathrm{H}_{2}$ with the boron sheet shows similarities to its interaction with graphene. We consider a graphene supercell similar to that of boron, which has of course the typical graphene structure without atoms in the centers of the hexagons. The calculated binding energy of $\mathrm{H}_{2}$ to graphene is then $25 \mathrm{meV}$. Likewise, the geometry of the adsorbed $\mathrm{H}_{2}$ molecule is very similar to that on the boron sheet, and the distance to the graphene plane is $3.24 \AA$. Our results on the graphene $-\mathrm{H}_{2}$ system are in accordance with earlier studies 46-48. We note that, although the binding of $\mathrm{H}_{2}$ to the boron sheet is slightly stronger than that to graphene, it is still too weak for a practical hydrogen storage application.

\subsubsection{Doping Boron Sheets with Alkali Metals}

Next, we consider deposition of AM atoms onto the boron sheet, with $\mathrm{AM}=\mathrm{Li}$, $\mathrm{Na}$, or K. To have a stable material at a reasonable temperature, it is essential that the metals strongly bind to the boron template. In addition, meaningful hydrogen gravimetric densities can be reached only if the AM atoms are separated far enough from each other so that each atom has sufficient space to bind hydrogen molecules. Placing a single AM atom in the unit cell shown in 7.1 leads to a lattice of AM atoms with a cell parameter of $5.06 \AA$. We vary the position of the AM atom on the boron sheet and relax all structures, as discussed in the previous section. The optimal position of all three lightweight AMs turns out to be over the center of the empty boron hexagon, as in 7.3. Upon relaxing, the unit cell parameters of the doped 

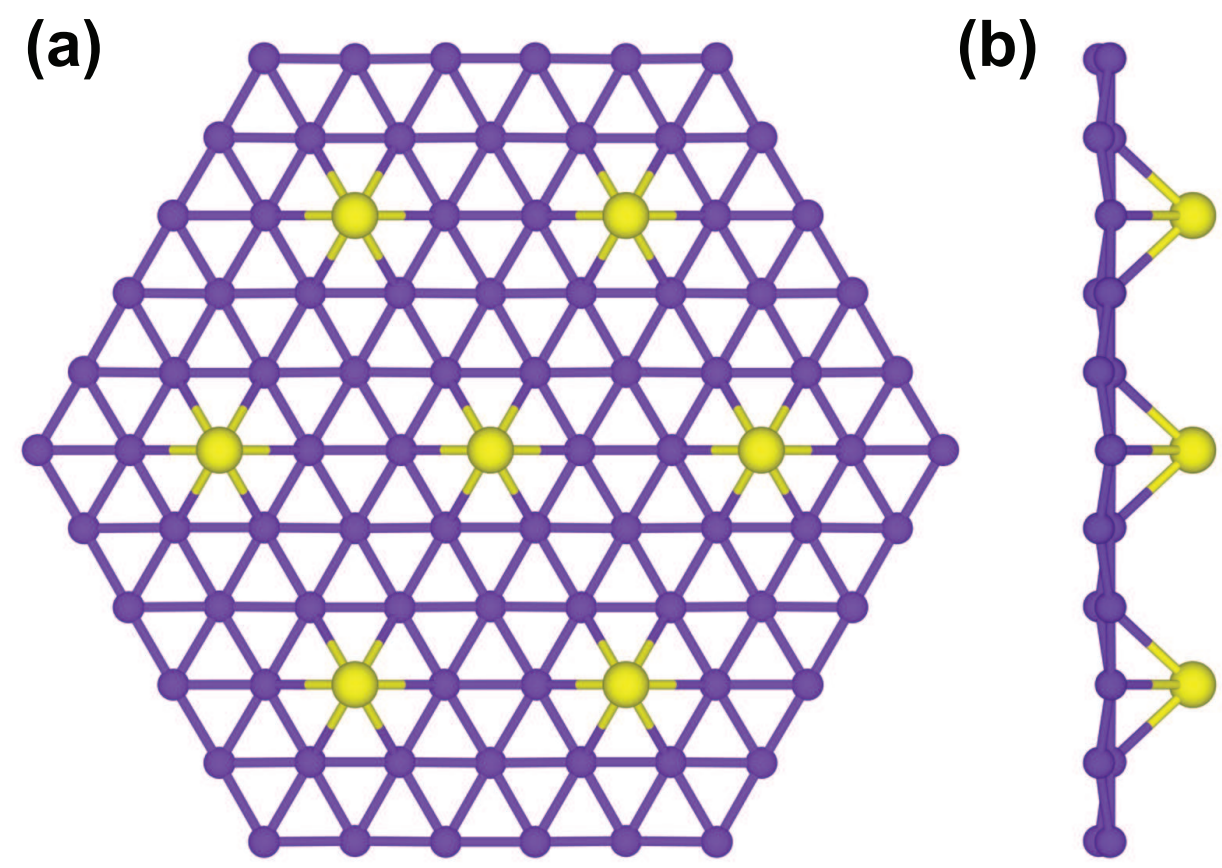

Figure 7.3: Optimized structure of a boron sheet doped with Li atoms (yellow spheres), shown from (a) the top and (b) the side. Boron sheets doped with other lightweight AMs have a similar geometry.

systems do not change noticeably. The optimized cell parameters are 5.08, 5.07, and $5.07 \AA$ for the $\mathrm{Li}, \mathrm{Na}$, and $\mathrm{K}$ doped boron sheet, respectively.

The binding energies of the AM atoms to the boron sheet are calculated according to

$$
E_{\mathrm{b}}=E_{\mathrm{AMbulk}}+E_{\mathrm{boron}}-E_{\mathrm{boronAM}}
$$

where $E_{\text {AMbulk }}$ is the total energy per atom of the AM bulk metal, $E_{\text {boronAM }}$ is the total energy per AM atom of the AM doped boron sheet, and $E_{\text {boron }}$ is the corresponding total energy of the boron sheet. The results are shown in 7.1 . For comparison, the binding energies $E_{\text {at }}$, calculated from eq 7.1 by replacing $E_{\mathrm{Ambulk}}$ with the energy of an isolated AM atom, are also given in this table. The numbers show that all three systems are thermodynamically stable with respect to separation into bulk metal and a boron sheet.

The equilibrium distance between the AM atoms and the boron plane, listed in 7.1, shows an increase going from Li to K, as might be expected. Electrostatic interactions 
Table 7.1: Calculated Binding Energies of Alkali Metals on a Boron Sheet (7.3) with Respect to Bulk Metal $\left(E_{\mathrm{b}}\right)$, Isolated Atoms $\left(E_{\mathrm{at}}\right)$, Equilibrium Distances to the Boron Plane $(d)$, and Bader Charges on the Alkali Atoms $(Q)$

\begin{tabular}{lcccc}
\hline \hline $\mathrm{AM}$ & $E_{\mathrm{b}}(\mathrm{eV})$ & $E_{\text {at }}(\mathrm{eV})$ & $d(\AA)$ & $Q(e)$ \\
\hline $\mathrm{Li}$ & 0.27 & 1.89 & 1.54 & +0.85 \\
$\mathrm{Na}$ & 0.02 & 1.11 & 2.26 & +0.60 \\
$\mathrm{~K}$ & 0.34 & 1.21 & 2.75 & +0.47 \\
\hline \hline
\end{tabular}

are dominant in binding hydrogen molecules to the AM atoms 12. Therefore, it is useful to make a Bader charge analysis to determine the effective charges on the AMs [49]. The Bader charges decrease going from Li to $\mathrm{K}$, which at first sight may seem surprising as the electronegativity of the AMs decreases along this series. However, comparing the nearest neighbor distances between the metal atoms on the boron surface with those in the bulk metals, one observes that the former are larger by 70, 40, and $10 \%$ for $\mathrm{Li}, \mathrm{Na}$, and $\mathrm{K}$, respectively. This means that the Li atoms on the boron sheet are relatively isolated, but the $\mathrm{K}$ atoms are still in relatively close contact. In this configuration, a Li atom is bonded to the boron sheet and the binding has a large ionic contribution, whereas a $\mathrm{K}$ atom is bonded to the boron sheet and to other $\mathrm{K}$ atoms with a mix of ionic and metallic bonding. Clearly, the binding of a $\mathrm{Na}$ atom is intermediate between these two situations.

In principle, one might increase the distance between the $\mathrm{K}$ atoms by decreasing their concentration on the boron sheet. However, $\mathrm{K}$ is already the heaviest element of the AM series considered here, and a reduction of their concentration increases the number of boron atoms per metal atom. This would seriously decrease the gravimetric hydrogen density, since the amount of hydrogen adsorbed depends on the metal atoms only. In conclusion, since Li is the lightest element, binds strongly to the boron sheet, and discharges almost completely upon deposition, it is the best candidate as a dopant for hydrogen storage purposes.

Since the Li atoms on the boron carry an effective positive charge, one may expect a net repulsion between these atoms. We consider the binding energy $E_{\mathrm{b}}$, eq 7.1 , and Bader charge $Q$ as a function of the Li concentration. The results are given in 7.2 . In the concentration we have considered so far, the $\mathrm{Li}-\mathrm{Li}$ nearest neighbor distance is $5.08 \AA$. Doubling the boron surface unit cell in each direction and occupying only one empty hexagon by a Li atom decreases the concentration by a factor of 4 and decreases the $\mathrm{Li}-\mathrm{Li}$ nearest neighbor distance by a factor of 2 . Occupying not only 
Table 7.2: Calculated Binding Energies with Respect to Bulk Metal $\left(E_{\mathrm{b}}\right)$ and Bader Charges $(Q)$ of $\mathrm{Li}$ on a Boron Sheet at Various Concentrations, Represented by the Nearest Neighbor Metal-Metal Distance $d_{\mathrm{nn}}$.

\begin{tabular}{ccc}
\hline \hline$d_{\mathrm{nn}}(\AA)$ & $E_{\mathrm{b}}(\mathrm{eV})$ & $Q(e)$ \\
\hline 10.16 & 0.45 & +0.88 \\
5.08 & 0.27 & +0.85 \\
2.93 & 0.16 & +0.75 \\
\hline \hline
\end{tabular}

all empty, but also all filled boron hexagons increases the Li concentration by a factor of 3. The Li-Li nearest neighbor distance is then multiplied $1 / \sqrt{(3)}$. The binding energy indeed decreases with increasing concentration, confirming the net repulsion between the Li atoms. There is a corresponding slight decrease of the Bader charge on the Li atoms. Note however that, at all concentrations studied, the system is stable against phase separation into a sheet carrying a lower concentration of Li and bulk $\mathrm{Li}$. The Li concentration can therefore be controlled by the amount of Li dispersed on the boron sheet.

It is interesting to compare the Li doping of the boron sheet to that of a similar system, namely, Li doping of graphene, which has been proposed as a suitable hydrogen storage system [10, 11]. We use a similar unit cell as for the boron sheet, containing six carbon atoms and three hexagons, one of which contains a $\mathrm{Li}$ atom. After relaxation, the lattice parameter of the graphene-Li cell is $4.28 \AA$, whereas the boron-Li cell parameter is slightly larger $(5.08 \AA)$. We find that the binding of $\mathrm{Li}$ atoms to graphene is considerably weaker than the binding to the boron sheet. On graphene, the binding energy $E_{\mathrm{b}}=-0.61 \mathrm{eV}$. Obviously, a negative value of the binding energy indicates that this system is thermodynamically unstable with respect to bulk $\mathrm{Li}$ and graphene, in contrast to the boron-Li system, cf. 7.1 .

Moreover, a Bader analysis carried out on the graphene-Li system reveals that only $0.64 e$ is removed from $\mathrm{Li}$ atoms after deposition, which is significantly smaller than the case of the boron-Li system. These results suggest that the boron sheet is a more promising candidate for lightweight AM doping than pure graphene.

\subsubsection{Mobility of Alkali Metal Atoms}

The stability of a material such as a doped boron or graphene sheet also depends on the mobility of the AM atoms on the sheet. Within the transition state theory model, the most important parameter determining the mobility is the energy barrier 
(a)

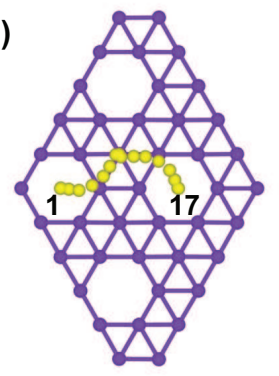

(b)

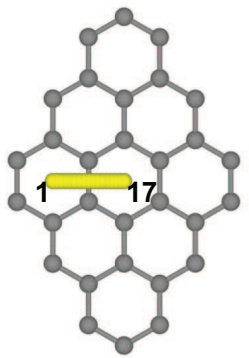

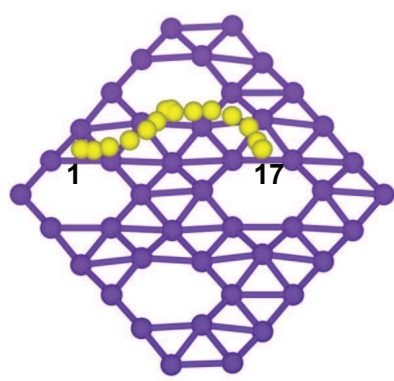

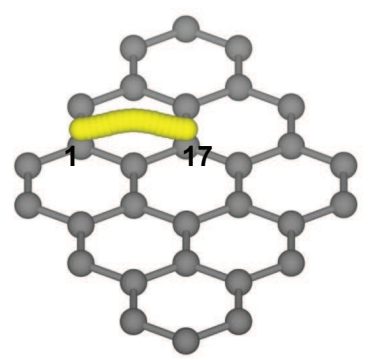

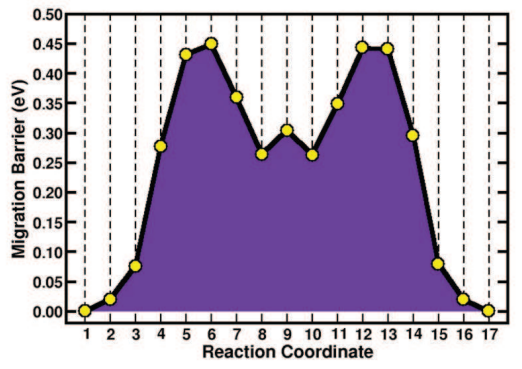

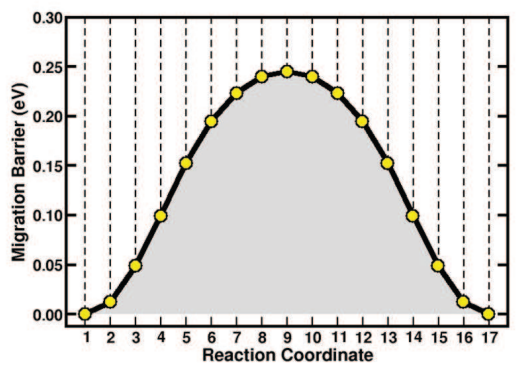

Figure 7.4: Diffusion of a Li atom over a (a) boron sheet and (b) graphene. The lowest energy diffusion paths are shown in top and perspective views. Calculated energy profiles along the paths are plotted on the right-hand side. The yellow dots in the plot correspond to the positions shown on the left-hand side. The diffusion barriers are 0.45 and $0.25 \mathrm{eV}$ on the boron and graphene, respectively.

for diffusion. We determine the diffusion path of a single $\mathrm{Li}$ atom on the boron sheet using the nudged elastic band method [50 52]. The calculations are carried out using a $2 \times 2$ supercell with 32 boron atoms, where one empty hexagon is occupied by a Li atom. A string of 17 images is chosen to model the diffusion path between two empty hexagons, as shown in 7.4 a. Positions over the centers of the empty hexagons represent absolute energy minima, and positions over the boron atoms at the centers of the other hexagons represent secondary adsorption minima. An optimal path runs over B atoms, and along B-B bonds, as shown in $7.4 \mathrm{a}$, with maxima over B-B bond centers. The calculated diffusion barrier is $0.45 \mathrm{eV}$. The lowest energy path for diffusion between two energy minima corresponding to empty hexagons proceeds via a secondary minimum over a filled hexagon, giving rise to a curved path. The simpler direct path between two empty hexagons involves an energy barrier of 0.55 $\mathrm{eV}$ and is therefore less likely.

We can compare this to diffusion of a $\mathrm{Li}$ atom over graphene. Positions over the 
Table 7.3: Calculated Binding Energies of Alkali Metals on a Boron Sheet Where Both Sides of the Sheet are Functionalized (7.3), with Respect to Bulk Metal $\left(E_{\mathrm{b}}\right)$ and Isolated Atoms $\left(E_{\mathrm{at}}\right)$, Equilibrium Distances to the Boron Plane $(d)$, Bader Charges on the Alkali Atoms $(Q)$, Hydrogen Binding Energies $\left(E_{\mathrm{H}_{2}}\right)$, and Gravimetric Hydrogen densities of the $\mathrm{B}_{8} \mathrm{AM}_{2}$ Systems.

\begin{tabular}{lcccccc}
\hline \hline $\mathrm{AM}$ & $E_{\mathrm{b}}(\mathrm{eV})$ & $E_{\mathrm{at}}(\mathrm{eV})$ & $d(\AA)$ & $Q(e)$ & $E_{\mathrm{H}_{2}}(\mathrm{eV})$ & $\mathrm{H}_{2}(\mathrm{wt} \%)$ \\
\hline $\mathrm{Li}$ & 0.18 & 1.80 & 1.62 & +0.82 & $0.35-0.15$ & 10.75 \\
$\mathrm{Na}$ & 0.02 & 1.11 & 2.28 & +0.60 & $0.13-0.07$ & 8.36 \\
$\mathrm{~K}$ & 0.35 & 1.22 & 2.75 & +0.47 & 0.06 & 2.39 \\
\hline \hline
\end{tabular}

centers of the carbon hexagons represent the absolute energy minima. A Li atom diffuses over a $\mathrm{C}-\mathrm{C}$ bond following a simple path, with a maximum over a $\mathrm{C}-\mathrm{C}$ bond center, as shown in 7.4b. The calculated diffusion barrier is $E_{\mathrm{d}}=0.25 \mathrm{eV}$. Consequently, Li atoms on graphene are quite mobile. Using an Arrhenius expression for the jump rate $\nu=\nu_{0} \exp \left[E_{\mathrm{d}} / k T\right]$ with a typical attempt frequency of $\nu_{0} \approx 10^{12}$ $\mathrm{Hz}$, one obtains $\nu \approx 10^{8} \mathrm{~Hz}$ at room temperature and $\nu \approx 1 \mathrm{~Hz}$ at $T=105 \mathrm{~K}$. Because of their negative binding energy with respect to bulk $\mathrm{Li}$, as discussed in the previous section, there is a thermodynamic driving force for Li atoms to cluster, which would seriously hamper the use of doped graphene as a hydrogen storage material [10, 11]. It is, however, possible to use graphene as a template for polylithiated molecules to create a stable storage material [12].

The diffusion barrier, $E_{\mathrm{d}}=0.45 \mathrm{eV}$, for Li atoms on the boron sheet is substantially larger than that on graphene. Using the same Arrhenius expression as above, a jump rate of $\nu \approx 1 \mathrm{~Hz}$ is obtained at $T=190 \mathrm{~K}$. At room temperature, individual Li atoms are still mobile $\left(\nu \approx 10^{4} \mathrm{~Hz}\right)$, but the formation bulk Li metal is thermodynamically unfavorable because of the high binding energy of the Li atoms on the boron sheet. The energy difference between the minimum energy sites of a $\mathrm{Li}$ atom (over the centers of empty boron hexagons) and the secondary minima is $0.3 \mathrm{eV}$. It means that at room temperature only the minimum energy sites are occupied. Since the $\mathrm{Li}$ atoms carry a substantial effective charge, there is in addition no driving force for clustering of metal atoms on the sheet. The minimum energy sites on the boron sheet form a regular lattice with a spacing of $5.08 \AA$ between the lattice points, which forms the ideal template for creating a stable lattice of $\mathrm{Li}$ atoms. 

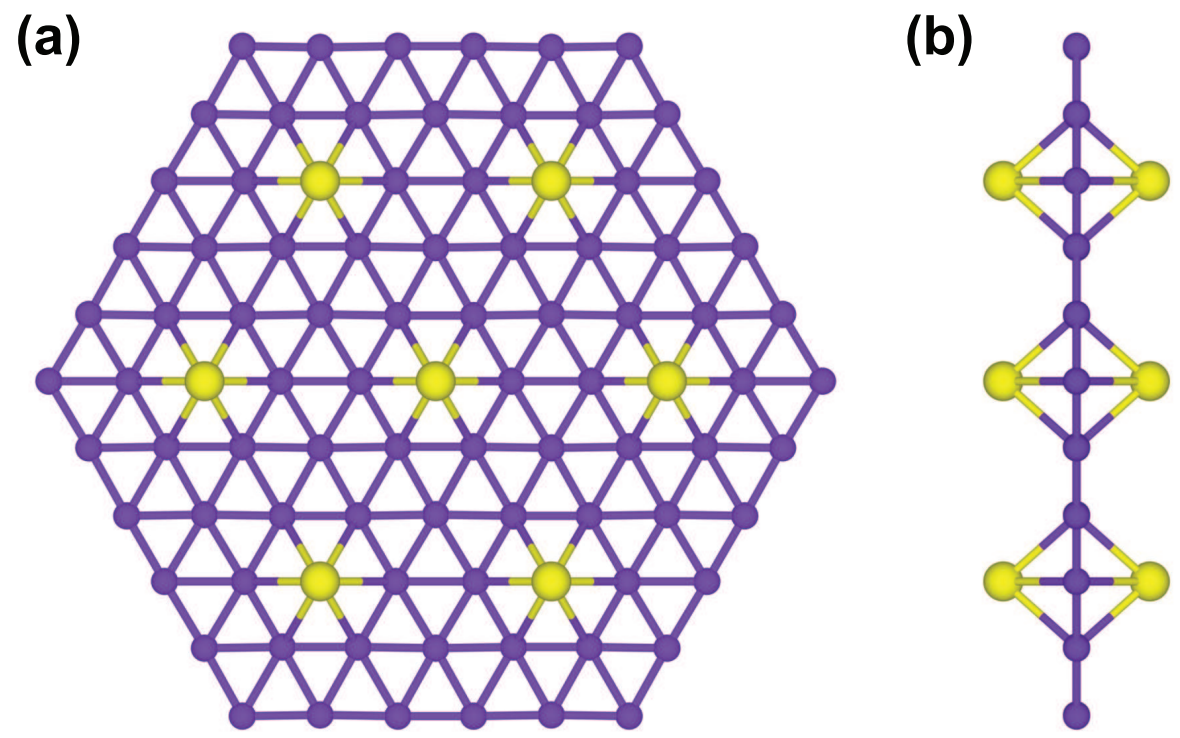

Figure 7.5: Optimized structure of a boron sheet doped with Li atoms on both sides, shown from (a) the top and (b) the side. Boron sheets doped with other lightweight AMs have a similar geometry.

\subsubsection{Hydrogen Adsorption on Alkali Doped Boron Sheets}

Starting from the AM lattice on the boron sheet, as discussed in the previous sections, we find in fact that both faces of the boron lattice can be functionalized with AMs, as depicted in 7.5. The chemical formula of the AM doped boron unit cells is then $\mathrm{B}_{8} \mathrm{AM}_{2}$ and the optimized lattice constants are 5.10, 5.08, and $5.08 \AA$ for the $\mathrm{AM}=\mathrm{Li}, \mathrm{Na}$, and $\mathrm{K}$, respectively. The optimized structures have mirror symmetry with respect to the boron plane. The binding energies of the AM atoms, their distances to the boron plane, and their Bader charges are given in 7.3 . A comparison to the data of 7.1 demonstrates that there is very little interaction between the AM atoms located above and below the boron sheet. Our general observations for AM doping remain valid; i.e., also for the doubly functionalized boron sheet, we have a stable lattice of effectively charged AM atoms.

Electrostatic and polarization interactions contribute most to the binding between the AM atoms and hydrogen molecules [12]. One may therefore expect that the effective charges on the metal atoms after deposition onto the boron sheet determines the strength of the interaction between the host material and the hydrogen molecules. 

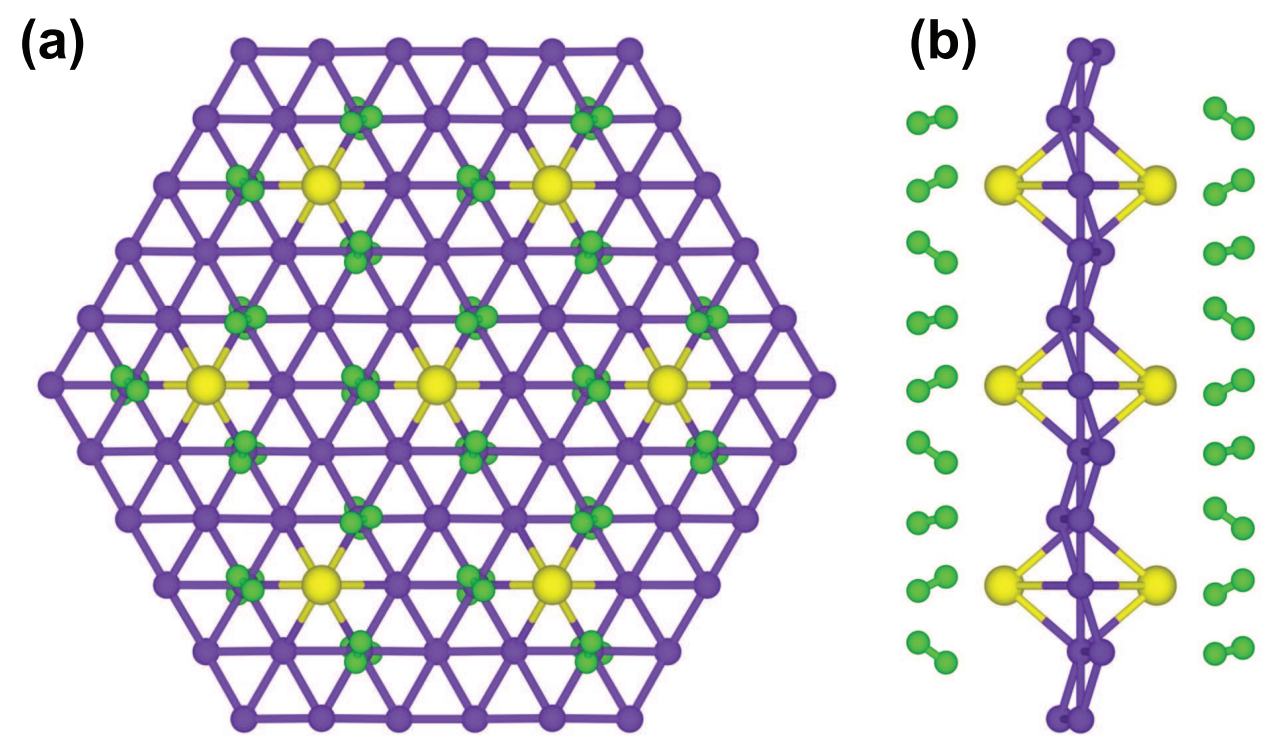

Figure 7.6: The optimized geometry of the Li doped boron sheet after full hydrogenation, shown from (a) the top and (b) the side. Three $\mathrm{H}_{2}$ molecules surround each Li atom.

These charges decrease along the series $\mathrm{Li}, \mathrm{Na}$, and $\mathrm{K}$, as shown by 7.3 . The binding energies of hydrogen molecules to the AM doped boron sheet follow this trend. The first two $\mathrm{H}_{2}$ molecules bind to $\mathrm{B}_{8} \mathrm{Li}_{2}$ with a binding energy of $0.35 \mathrm{eV} / \mathrm{H}_{2}$ (one $\mathrm{H}_{2}$ binds to the top $\mathrm{Li}$, and one to the bottom $\mathrm{Li}$ ). The corresponding numbers for $\mathrm{Na}$ and $\mathrm{K}$ are significantly smaller, i.e., 0.13 and $0.06 \mathrm{eV} / \mathrm{H}_{2}$, respectively.

Each $\mathrm{Li}$ atom in this structure can capture up to three $\mathrm{H}_{2}$ molecules, but the average binding energy then drops to $0.15 \mathrm{eV} / \mathrm{H}_{2}$. In the fully loaded case, the $\mathrm{B}_{8} \mathrm{Li}_{2}$ compound then contains 10.7 wt \% hydrogen, and the chemical formula of the unit cell is $\mathrm{B}_{8} \mathrm{Li}_{2}\left(\mathrm{H}_{2}\right)_{6}$. The optimized geometry is shown in 7.6. The centers of all the $\mathrm{H}_{2}$ molecules surrounding the Li atoms are located at an equivalent distance of $2.35 \AA$ from the metal atoms. These results are in line with the interactions of $\mathrm{H}_{2}$ molecules with isolated AM cations [14, 24]. The maximum number of $\mathrm{H}_{2}$ molecules that can be gathered around a metal center is determined by the Coulomb interactions that develop between $\mathrm{Li}-\mathrm{H}_{2}$ and $\mathrm{H}_{2}-\mathrm{H}_{2}$, and by steric effects.

The $\mathrm{B}_{8} \mathrm{Na}_{2}$ system can also capture three $\mathrm{H}_{2}$ molecules per metal. The average binding energy for the $\mathrm{H}_{2}$ molecules then drops to $0.07 \mathrm{eV} / \mathrm{H}_{2}$, which is still larger than the binding between a $\mathrm{H}_{2}$ molecule and a bare boron sheet. In contrast, the 


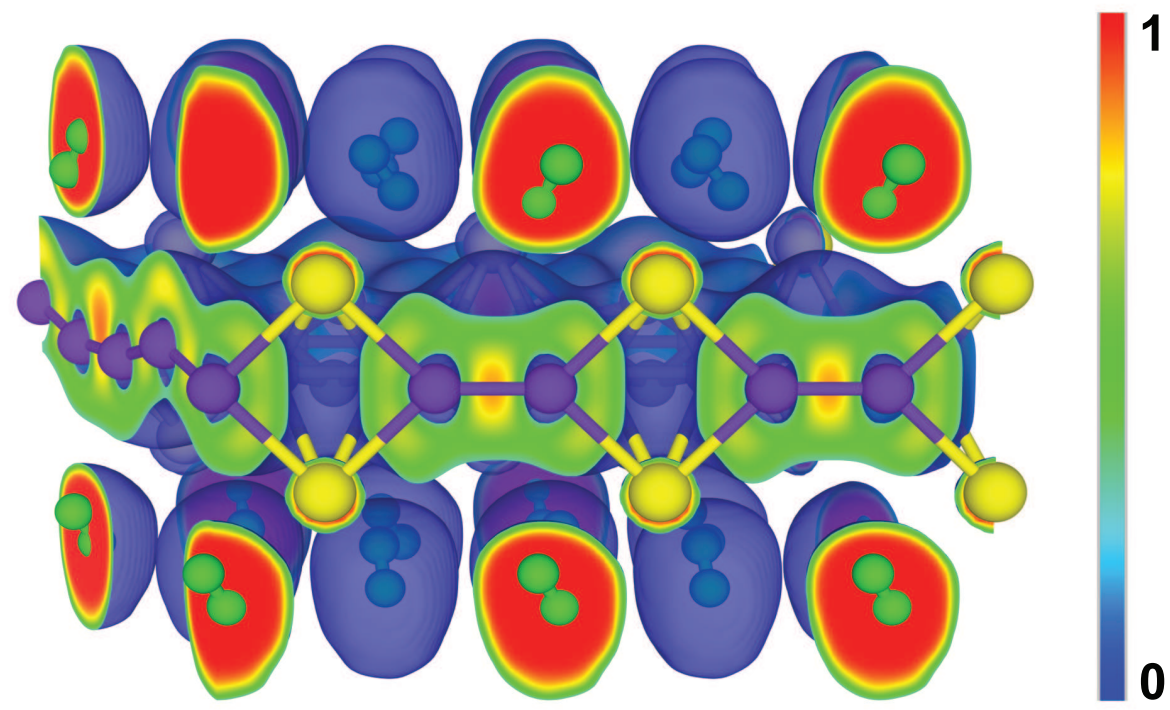

Figure 7.7: Electron localization function of the boron-Li system at its fully hydrogenated state. The electrons are localized at the $\mathrm{H}_{2}$ molecules and around the boron sheet, where the bonding is covalent. The positively charged Li ions are depleted from electrons.

$\mathrm{B}_{8} \mathrm{~K}_{2}$ system is capable of capturing only a single $\mathrm{H}_{2}$ molecule per $\mathrm{K}$ atom with a low binding energy of $0.06 \mathrm{eV} / \mathrm{H}_{2}$. It is clear that, if compared to the $\mathrm{Li}$ doped system, the higher electron densities on the $\mathrm{Na}$ and $\mathrm{K}$ atoms result in weaker interactions with $\mathrm{H}_{2}$ molecules. The $\mathrm{Li}$ doped boron sheet is the best option for hydrogen storage.

It is interesting to note that the amount of charge on the $\mathrm{Li}$ atoms, as a consequence of their interaction with the boron lattice, stays almost constant during the consecutive addition of $\mathrm{H}_{2}$ molecules. The $\mathrm{H}-\mathrm{H}$ bonds of the adsorbed $\mathrm{H}_{2}$ molecules are only elongated by $\sim 1.5 \%$ as compared to an isolated $\mathrm{H}_{2}$ molecule, which illustrates that the interaction between $\mathrm{H}_{2}$ molecules and the $\mathrm{Li}$ atoms is mostly electrostatic and not chemical bonding.

The calculated electron localization function for the $\mathrm{B}_{8} \mathrm{Li}_{2}\left(\mathrm{H}_{2}\right)_{6}$ system is shown in 7.7. The Li atoms are depleted, consistent with our Bader analysis. Hydrogens stay in molecular form and are surrounded by their localized electron clouds. There is no direct indication of an orbital interaction between the Li atoms and the hydrogens. Moreover, the pattern of the covalent bonding within the boron lattice is not significantly altered by the $\mathrm{Li}$ atoms or the $\mathrm{H}_{2}$ molecules. 


\subsection{Conclusions}

We study the planar boron $\alpha$-sheet as a physisorption template for hydrogen storage. Direct physisorption of $\mathrm{H}_{2}$ on a boron sheet gives a binding energy of 0.05 eV. Alkali metal (AM) atoms can be dispersed on the boron sheet to increase the hydrogen binding energies. The boron sheet interacts strongly with AM atoms, and the doped systems are thermodynamically stable with respect to clustering of metal atoms. Moreover, the unique geometry of the boron plane provides a natural lattice for the metal atoms whose nearest neighbor distance can be tuned by varying their concentration. In particular, $\mathrm{Li}$ is found to be a promising doping element for hydrogen storage. The strong interaction between the boron sheet and the Li atoms results in an almost complete transfer of the Li valence electrons to the boron sheet. Electrostatic interactions between the well-exposed Li atoms and the $\mathrm{H}_{2}$ molecules then lead to an average binding energy of $0.15 \mathrm{eV} / \mathrm{H}_{2}$. The system physisorbs up to a maximum of $10.7 \mathrm{wt} \%$ hydrogen. In contrast, AM atoms dispersed on graphene are generally unstable with respect to the bulk metal, which suggests that AM doped nanostructures based upon boron are a much better starting point for hydrogen storage than their carbon based counterparts.

\section{Acknowledgments}

This work is part of the research programs of "Advanced Chemical Technologies for Sustainability (ACTS)" and the "Stichting voor Fundamenteel Onderzoek der Materie (FOM)". The use of supercomputer facilities was sponsored by the "Stichting Nationale Computerfaciliteiten (NCF)". These institutions are financially supported by "Nederlandse Organisatie voor Wetenschappelijk Onderzoek (NWO)".

\section{Bibliography}

[1] See the special issue Toward a Hydrogen Economy, by R. Coontz and B. Hanson, Science 305, 957 (2004).

[2] L. Schlapbach and A. Züttel, Nature 414, 353 (2001).

[3] M. J. van Setten, G. A. de Wijs, V. A. Popa, and G. Brocks, Phys. Rev. B 72, 073107 (2005).

[4] S. Er, D. Tiwari, G. A. de Wijs, and G. Brocks, Phys. Rev. B 79, 024105 (2009). 
[5] M. J. van Setten, G. A. de Wijs, and G. Brocks, Phys. Rev. B 76, 075125 (2007).

[6] R. Gremaud et al., Adv. Mater. 19, 2813 (2007).

[7] S. K. Bhatia and A. L. Myers, Langmuir 22, 1688 (2006).

[8] T. Yildirim and S. Ciraci, Phys. Rev. Lett. 94, 175501 (2005).

[9] E. Durgun, S. Ciraci, and T. Yildirim, Phys. Rev. B 77, 85405 (2008).

[10] W. Q. Deng, X. Xu, and W. A. Goddard, Phys. Rev. Lett. 92, 166103 (2004).

[11] C. Ataca, E. Aktürk, S. Ciraci, and H. Ustunel, Appl. Phys. Lett. 93, 043123 (2008).

[12] S. Er, G. A. de Wijs, and G. Brocks, J. Phys. Chem. C 113, 8997 (2009).

[13] Q. Sun, P. Jena, Q. Wang, and M. Marquez, J. Am. Chem. Soc. 128, 9741 (2006).

[14] K. R. S. Chandrakumar and S. K. Ghosh, Nano Lett. 8, 13 (2008).

[15] Q. Sun, Q. Wang, and P. Jena, Appl. Phys. Lett. 94, 013111 (2009).

[16] P. Chen, X. Wu, J. Lin, and K. L. Tan, Science 285, 91 (1999).

[17] C. Liu et al., Science 286, 1127 (1999).

[18] R. Yang, Carbon 38, 623 (2000).

[19] E. Lee, Y. Kim, Y. Jin, and K. Chang, Phys. Rev. B 66, 73415 (2002).

[20] A. Blomqvist, C. M. Araujo, P. Srepusharawoot, and R. Ahuja, Proc. Natl. Acad. Sci. U.S.A. 104, 20173 (2007).

[21] S. S. Han, W. Q. Deng, and W. A. Goddard, Angew. Chem., Int. Ed. 46, 6289 (2007).

[22] A. Mavrandonakis, E. Tylianakis, A. K. Stubos, and G. E. Froudakis, J. Phys. Chem. C 112, 7290 (2008).

[23] E. Klontzas, A. Mavrandonakis, E. Tylianakis, and G. Froudakis, Nano Lett. 8, 1572 (2008).

[24] R. C. Lochan and M. Head-Gordon, Phys. Chem. Chem. Phys. 8, 1357 (2006).

[25] K. S. Novoselov et al., Science 306, 666 (2004). 
[26] A. Geim and A. MacDonald, Phys. Today 60, 35 (2007).

[27] E. L. Muetterties, The chemistry of boron and its compounds (New York: John Wiley \& Sons, Inc., 1967).

[28] H. Werheit, Landolt-Börnstein: Numerical Data and Functional Relationships in Science and Technology - New Series (Berlin: Springer, 2000).

[29] H. Tang and S. Ismail-Beigi, Phys. Rev. Lett. 99, 115501 (2007).

[30] K. C. Lau and R. Pandey, J. Phys. Chem. C 111, 2906 (2007).

[31] K. C. Lau and R. Pandey, J. Phys. Chem. B 112, 10217 (2008).

[32] X. Yang, Y. Ding, and J. Ni, Phys. Rev. B 77, 041402 (2008).

[33] D. L. Prasad and E. D. Jemmis, Phys. Rev. Lett. 100, 165504 (2008).

[34] Q. B. Yan, X. L. Sheng, Q. R. Zheng, L. Z. Zhang, and G. Su, Phys. Rev. B 78, 20 (2008).

[35] T. Baruah, M. R. Pederson, and R. R. Zope, Phys. Rev. B 78, 045408 (2008).

[36] A. K. Singh, A. Sadrzadeh, and B. I. Yakobson, Nano Lett. 8, 1314 (2008).

[37] K. C. Lau, R. Orlando, and R. Pandey, J. Phys.: Condens. Matter 20, 125202 (2008).

[38] Y. Ding, X. Yang, and J. Ni, Appl. Phys. Lett. 93, 043107 (2008).

[39] G. Kresse and J. Hafner, Phys. Rev. B 47, 558 (1993).

[40] G. Kresse and J. Furthmüller, Phys. Rev. B 54, 11169 (1996).

[41] J. P. Perdew et al., Phys. Rev. B 46, 6671 (1992).

[42] P. E. Blöchl, Phys. Rev. B 50, 17953 (1994).

[43] G. Kresse and D. Joubert, Phys. Rev. B 59, 1758 (1999).

[44] H. J. Monkhorst and J. D. Pack, Phys. Rev. B 13, 5188 (1976).

[45] P. E. Blöchl, O. Jepsen, and O. K. Andersen, Phys. Rev. B 49, 16223 (1994).

[46] T. Heine, L. Zhechkov, and G. Seifert, Phys. Chem. Chem. Phys. 6, 980 (2004).

[47] D. Henwood and J. D. Carey, Phys. Rev. B 75, 245413 (2007). 
[48] N. Park, S. Hong, G. Kim, and S. H. Jhi, J. Am. Chem. Soc. 129, 8999 (2007).

[49] G. Henkelman, A. Arnaldsson, and H. Jónsson, Comput. Mater. Sci. 36, 354 (2006).

[50] H. Jónsson, G. Mills, and K. W. Jacobsen, Nudged Elastic Band Method for Finding Minimum Energy Paths of Transitions, in Classical and Quantum Dynamics in Condensed Phase Simulations (Singapore: World Scientific, 1998).

[51] G. Henkelman and H. Jónsson, J. Chem. Phys. 113, 9978 (2000).

[52] D. Sheppard, R. Terrell, and G. Henkelman, J. Chem. Phys. 128, 134106 (2008). 


\section{Chapter 8}

\section{Lightweight metal doping of metal-organic frameworks: Implications for hydrogen storage}

\subsection{Introduction}

Metal organic frameworks (MOFs) are low density crystalline materials with exceptionally high porosity [1. Recent advancements in MOF synthesis are promising their widespread usage as gas storage systems, particularly hydrogen [2 15]. Available MOF systems, however, are known to bind hydrogen molecules very weakly, therefore requiring massive operating pressures and cryogenic conditions. Recent theoretical studies on Li doped MOF systems report significant improvements on molecular hydrogen uptake capacities near ambient conditions [16-18]. In these studies, however, Li atoms are modeled to bind to the aromatic linker units. Here, we report that lightweight alkali metals $(\mathrm{AM}=\mathrm{Li}, \mathrm{Na}$, and $\mathrm{K})$ and alkaline earth metals $(\mathrm{AEM}=\mathrm{Be}, \mathrm{Mg}$, and $\mathrm{Ca})$ favorably bind to the oxide connector units in MOF-5. At higher metal doping concentrations the connector structure is reconstructed so that $\mathrm{Zn}$ ions are partially replaced by the doping metal ions, eventually ceasing the network porosity. Altering the aromatic linker to generate more favorable sites for 
the doping metals has found no use. A similar scenario is predicted for remaining members of MOF family built with oxides of other transition metal ions.

\subsection{Computational Methods}

Optimization of the geometry and calculation of total energy and forces are performed with first principles calculations at the level of density functional theory (DFT), using the VASP code 19 21. Generalized gradient approximation (GGA) of DFT in the form of PW91 functional 22] is used for the exchange and correlation potentials. Projector augmented wave (PAW) formalism is adopted and the cutoff kinetic energy for the plane wave basis is set to $400 \mathrm{eV}$ [23, 24]. The Brillouin zone (BZ) is sampled at the $\Gamma$-point only due to a large unit cell. Shape and volume of the cells are conserved while the atomic coordinates were subject to change without any symmetry constraints. Convergence criterions for calculation of self consistent total energy and the structural optimizations were $10^{-4} \mathrm{eV}$ difference between two consecutive electronic steps and $0.02 \mathrm{eV} / \AA$ residual force on each atom, respectively.

\subsection{Results}

We first determine the strength of interaction between a single metal atom and the MOF-5 structure ( 1 at \% dopant). The lowest energy structures with AMs are the ones where the dopants are positioned at connector sites, between oxygen atoms of neighboring $\mathrm{BDC}^{2-}(=1,4$-benzenedicarboxylate) linkers. These geometries are lower in energy than the metals occupying the center of linker carbon rings by $0.31,0.31$ and $0.19 \mathrm{eV} /$ cell for $\mathrm{Li}, \mathrm{Na}$, and $\mathrm{K}$ dopants, respectively. In the case of AEM doping, we find that both $\mathrm{Be}$ and $\mathrm{Ca}$ are located at the connector sites, whereas a single neutral $\mathrm{Mg}$ atom interacts very weakly with MOF-5 on both sites. The optimized geometries for Be and $\mathrm{Ca}$, however, are different. Be atom, prefers a similar position to that of AM atoms. Ca, on the other hand, favors a geometry where it stays intact with seven oxygen atoms, six from three $\mathrm{BDC}^{2-}$ linkers and one from the tetrahedral $\left[\mathrm{Zn}_{4} \mathrm{O}\right]^{6+}$. The presence of a single $\mathrm{Ca}$ atom inside the MOF-5 unit cell has greater influence on the modification of the zinc oxide connector site than the rest of the doping materials considered here.

We further investigate the effects of $\mathrm{AM}(\mathrm{AEM})$ doping on the MOF-5 system by considering a situation with increased doping concentration. In principle, when both faces of the $\mathrm{BDC}^{2-}$ linkers are functionalized twelve metal ions would be accommodated within the unit cell ( $\sim 10$ at $\%$ dopant). Here, we compare the relative stability 


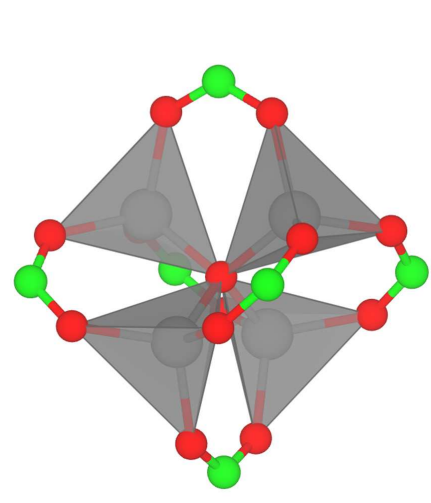

$\mathrm{Zn}_{4} \mathrm{O}\left(\mathrm{CO}_{2}\right)_{6}$

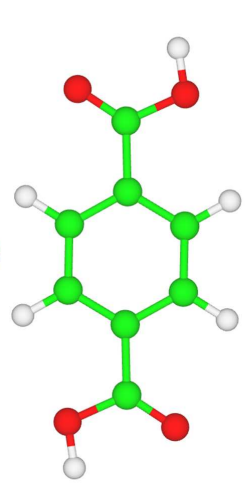

C6

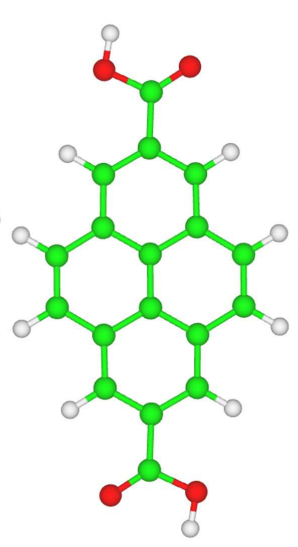

C16

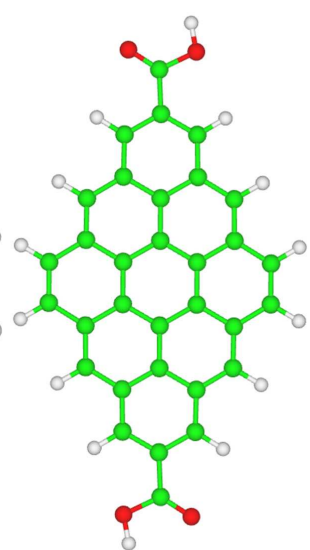

C30

Figure 8.1: Connector geometry in MOF-5 (left figure), and linkers used in this study. Zinc, carbon, oxygen, and hydrogen atoms are shown as grey, green, red and white spheres, respectively.

of the two cases. In the first case, prior to relaxation all the twelve metal atoms are placed at a bonding distance above and below the hexagonal centers of six $\mathrm{BDC}^{2-}$ units. In the second case, all the metal atoms are placed around one of the two oxide connector sites inside the unit cell. Same initial geometries are used to simulate the effects of doping with $\mathrm{AM}(\mathrm{AEM})$ ions.

For each individual dopant, total energy differences between the optimized geometries of the first and the second cases are given in Table 8.1. It is clear that the entire set of lightweight AMs or AEMs thermodynamically favor the second case, in which they interact closely with the oxygen atoms of the MOF-5 system. This is in contrast to the earlier studies on the same system where only organic linker sites were considered for atomic Li adsorption [16 18. Our results obtained from heavy doping are in connection with the single atom doped systems. Moreover, (except for $\mathrm{Na}$ and K) increasing doping concentration enlarges the total energy gap between the two cases, in favor of metal clustering around oxide connector sites. Particularly for Li doping, the energy difference is $7.66 \mathrm{eV} /$ cell (or $0.64 \mathrm{eV} / \mathrm{Li}$ ) (Table 8.1). The increment in total energy difference compared to the single atom doped system is due to a structural change around the connector site. In the relaxed structure, one of the $\mathrm{Zn}$ ions is found to be removed form the tetrahedral $\left[\mathrm{Zn}_{4} \mathrm{O}\right]^{6+}$ unit and moved to the cell cavity, consequently central oxygen is now bonded to a Li atom (Fig 2). The rest of the AM atoms also are stabilized in the vicinity of oxygen atoms. For 
Table 8.1: Calculated binding energy values, Eb (eV/dopant), of metal dopants to the MOF-5 structure. Data corresponds to $\sim 1$ at $\%$ and 10 at $\%$ dopant concentration.

\begin{tabular}{lccccc}
\hline \hline \multirow{2}{*}{ Doping element } & \multicolumn{2}{c}{$\sim 1$ at $\%$} & & \multicolumn{2}{c}{$\sim 10$ at $\%$} \\
\cline { 2 - 3 } \cline { 5 - 6 } & @linker & @connector & & @linker & @ connector \\
$\mathrm{Li}$ & -1.328 & -1.640 & & -1.172 & -1.811 \\
$\mathrm{Na}$ & -0.699 & -1.013 & & -0.636 & -0.941 \\
$\mathrm{~K}$ & -1.096 & -1.283 & & -0.931 & -1.106 \\
$\mathrm{Be}$ & -0.167 & -1.107 & & -1.027 & -3.366 \\
$\mathrm{Mg}$ & -0.037 & -0.051 & & -0.643 & -1.356 \\
$\mathrm{Ca}$ & -1.323 & -1.520 & & -1.226 & -2.891 \\
\hline \hline
\end{tabular}

$\mathrm{Na}(\mathrm{K})$, total energy differences between the relaxed structures of linker and connector sites is $3.66(2.09) \mathrm{eV} /$ cell in favor of connector sites, but noticeably smaller than the Li doping scenario (Table 8.1). These values are in connection with the single atom doped system. Conservation of the $\left[\mathrm{Zn}_{4} \mathrm{O}\right]^{6+}$ geometry upon $\mathrm{Na}(\mathrm{K})$ doping together with the binding energy values implies that there is an energy barrier for structural modification. Earlier second group elements (Be, $\mathrm{Mg}$, and $\mathrm{Ca}$ ) also tend to cluster around the oxide connector positions. Interestingly, Zn ions are exchanged by all the AEMs, and accordingly, the cell geometry is modified. The results show that, increasing AEM doping concentrations, as in the case of Li doping, lead to a catastrophic restructuring of the geometry. It is therefore expected that the porosity of the 3-D system would be affected on the macro scale.

It is also important to know whether our conclusions on the MOF-5 will also hold for other MOF systems build with different organic linkers or metal oxide connectors. Isoreticular series of MOFs with $\mathrm{Zn}_{4} \mathrm{O}\left(\mathrm{CO}_{2}\right)_{6}$ connectors are already been successfully synthesized [7, 25. Here, as an example, we consider the interaction of a single neutral AM(AEM) atoms with three different molecules of various sizes, namely $\mathrm{C} 6$, C16 and C30 (Fig 1) (naming is according to the total number of ring carbons ,as in Ref.[16]). AMs are found to bind the $\mathrm{C} 30$ molecule the strongest, but with no remarkable improvement in binding energies compared to $\mathrm{C} 6$ molecule. For instance, in the case of $\mathrm{Li}$, the calculated binding energy to the $\mathrm{C} 30$ molecule is $\sim 50 \mathrm{meV}$ higher than to the C6 molecule. The binding energy value for the C6-Li system matches closely to that of one Li atom MOF-5 linker unit interaction. AEMs, on the other hand, 
(a)

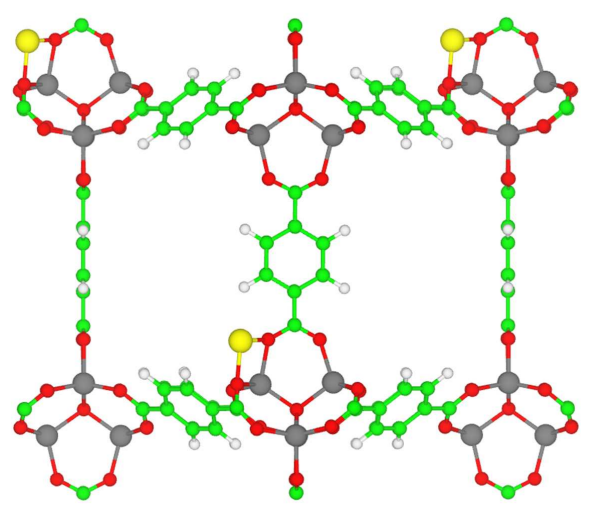

(b)

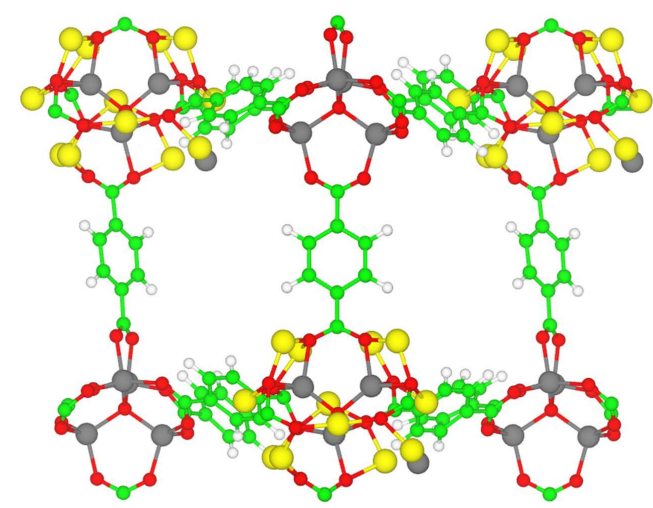

Figure 8.2: Optimized geometries of MOF-5 system upon $\sim 1$ at $\%$ (a), and $\sim 10$ at \% (b) Li (yellow spheres) doping at connector sites.

interact with the $\mathrm{C} 6$ molecule stronger than the other two molecules. Apart from weakly interacting $\mathrm{Be}$ and non-reacting $\mathrm{Mg}$, $\mathrm{Ca}$ seems to be the ideal dopant within the AEM group. To sum up, we confirm that altering the linker does not provide a viable solution. For all of the doping metal atoms, none of the organic linkers studied here were able to successfully generate an energetically favorable site compared to the $\mathrm{Zn}_{4} \mathrm{O}\left(\mathrm{CO}_{2}\right)_{6}$ connectors. Future synthesis of MOFs with high electron affinity linkers might open a new path for lightweight metal doping.

MOFs build with metal ions other than Zn have also drawn recent interest [7, 2629]. Such systems are not necessarily isostructural with MOF-5. It is therefore not straightforward for us to study all of the available MOF structures in here. However, we can still generalize our conclusions on the prototype MOF-5 structure to the other MOF systems build with different transition metal oxide connectors. The key point whether a MOF system would resist structural alterations upon $\mathrm{AM}(\mathrm{AEM})$ doping lies on the relative stability of the metal oxide units. In the bulk phase, oxides of lightweight $\mathrm{AM}(\mathrm{AEM})$ atoms are mostly more stable than the oxides of transition metal ions 30 32. Therefore, upon doing, most of the MOF systems with transition metal connector sites would be vulnerable to metal substitution reactions as observed in $\mathrm{MOF}-5$. 


\section{Acknowledgments}

This work is part of the research programs of "Advanced Chemical Technologies for Sustainability (ACTS)" and the "Stichting voor Fundamenteel Onderzoek der Materie (FOM)". The use of supercomputer facilities was sponsored by the "Stichting Nationale Computerfaciliteiten (NCF)". These institutions are financially supported by "Nederlandse Organisatie voor Wetenschappelijk Onderzoek (NWO)".

\section{Bibliography}

[1] O. Yaghi et al., Nature 423, 705 (2003).

[2] N. L. Rosi et al., Science 300, 1127 (2003).

[3] X. Zhao et al., Science 306, 1012 (2004).

[4] J. Rowsell, J. Eckert, and O. Yaghi, J. Am. Chem. Soc. 127, 14904 (2005).

[5] J. Rowsell, A. Millward, K. Park, and O. Yaghi, J. Am. Chem. Soc. 126, 5666 (2004).

[6] J. Rowsell, E. Spencer, J. Eckert, J. Howard, and O. Yaghi, Science 309, 1350 (2005).

[7] J. Rowsell and O. Yaghi, J. Am. Chem. Soc. 128, 1304 (2006).

[8] J. Rowsell and O. Yaghi, Angew. Chem., Int. Ed. 44, 4670 (2005).

[9] A. Wong-Foy, A. Matzger, and O. Yaghi, J. Am. Chem. Soc 128, 3494 (2006).

[10] S. S. Kaye, A. Dailly, O. M. Yaghi, and J. R. Long, J. Am. Chem. Soc 129, 14176 (2007).

[11] J. G. Vitillo et al., J. Am. Chem. Soc. 130, 8386 (2008).

[12] W. Zhou, H. Wu, and T. Yildirim, J. Am. Chem. Soc. 130, 15268 (2008).

[13] M. Dinca and J. R. Long, Angew. Chem., Int. Ed. 47, 6766 (2008).

[14] A. Dailly, J. Vajo, and C. Ahn, J. Phys. Chem. B 110, 1099 (2006).

[15] J. Liu et al., J. Phys. Chem. C 100, 2911 (2008).

[16] S. Han and W. Goddard III, J. Am. Chem. Soc. 129, 8422 (2007). 
[17] A. Blomqvist, C. M. Araujo, P. Srepusharawoot, and R. Ahuja, Proc. Natl. Acad. Sci. U.S.A. 104, 20173 (2007).

[18] A. Mavrandonakis, E. Tylianakis, A. K. Stubos, and G. E. Froudakis, J. Phys. Chem. C 112, 7290 (2008).

[19] G. Kresse and J. Hafner, Phys. Rev. B 47, 558 (1993).

[20] G. Kresse and J. Furthmüller, Phys. Rev. B 54, 11169 (1996).

[21] G. Kresse and J. Furthmüller, Comput. Mater. Sci. 6, 15 (1996).

[22] J. P. Perdew and Y. Wang, Phys. Rev. B 45, 13244 (1992).

[23] P. E. Blöchl, Phys. Rev. B 50, 17953 (1994).

[24] G. Kresse and D. Joubert, Phys. Rev. B 59, 1758 (1999).

[25] M. Eddaoudi et al., Science 295, 469 (2002).

[26] N. Rosi et al., J. Am. Chem. Soc. 127, 1504 (2005).

[27] H. Furukawa, J. Kim, N. Ockwig, M. OKeeffe, and O. Yaghi, J. Am. Chem. Soc. 130, 11650 (2008).

[28] P. Dietzel, Y. Morita, R. Blom, and H. Fjellvag, Angew. Chem., Int. Ed. 44, $6354(2005)$.

[29] P. Dietzel, B. Panella, M. Hirscher, R. Blom, and H. Fjellvåg, Chem. Commun. 2006, 959 (2006).

[30] O. Kubaschewski, C. B. Alcock, and P. J. Spencer, Mater Thermochemistry (Oxford: Pergamon Press, 1993).

[31] M. W. Chase, NIST-JANAF Thermochemical Tables (New York: American Chemical Society, 1998).

[32] L. Wang, T. Maxisch, and G. Ceder, Phys. Rev. B 73, 195107 (2006). 



\section{Chapter 9}

\section{Summary}

At the present time, a sustainable provision of energy is one of the greatest challenges for mankind. It requires ground-breaking scientific ideas, bold monetary investments and innovative public actions to initiate technological, economical and social transitions. Energy generated from sustainable sources has to be transported and stored in an efficient and ecologically friendly way. At this point, hydrogen is a vital ingredient in the sustainable energy scenarios. It is crucial to find safe, compact and cost effective methods for storing hydrogen to enhance the future realization of the sustainable energy economy. Due to the complexity of developing hydrogen storage systems, scientific research at a fundamental level is required. The present thesis is devoted to the first-principles study of the hydrogen storage properties of a set of promising bulk, nano and molecular systems.

The thesis starts with a brief introduction (Chapter 1) of the background of hydrogen as a fuel. The introduction chapter lists several conventional and novel hydrogen storage methods. Viable hydrogen storage technologies should be able to encompass all the storage objectives. Besides being safe and economically affordable, the storage material needs to be compact and lightweight. Accordingly, a promising storage material has to achieve meaningful gravimetric and volumetric hydrogen densities. It is, therefore, a necessity that the storage system is built to a great extend from lightweight chemical elements. The operability of storage materials depends hugely on their thermal and kinetic properties. Accordingly, the materials should allow for an efficient thermal management and fast hydrogen (un)loading rates. Moreover, the storage systems need to be durable over many hydrogen (un)loading cycles.

Chapter 2 summarizes the computational methodology by discussing the theoretical and the practical background of the present study. Density functional theory 
is described, which is the working horse of practical calculations. In an effort to simplify the solution of the complex physical problems a set of approximations are necessarily introduced. Pseudopotentials and plane-waves with a finite energy cutoff are the most notable of these approximations. The details of the calculations on the physical and the chemical properties of the materials are discussed at the end of the methodology chapter.

The computational results are presented in two parts. In Part - A, storage materials that are suited for storage of hydrogen in atomic form are considered. The main interest of Part $\mathrm{A}$ is devoted to the bulk systems of magnesium-dihydride $\left(\mathrm{MgH}_{2}\right)$ and its alloys with transition metals and aluminium or silicon. $\mathrm{MgH}_{2}$ is an inexpensive, and compact material that offers storing of hydrogen in suitable gravimetric and volumetric densities. However, it suffers from slow (de)hydrogenation kinetics and a high thermodynamic stability. Alloying $\mathrm{MgH}_{2}$ with lightweight transition metals (TMs) aims at enhancing the kinetic and the thermodynamic properties. As shown in Chapter 3. TM-alloying $(\sim 20$ at. \%) initiates a phase transition from the original $\mathrm{MgH}_{2}$ phase to a fluorite phase, resulting in significant improvements in the (de)hydrogenation kinetics. The thermodynamic properties of the new materials with high magnesium contents, however, are not improved sufficiently. In Chapter 4, possible disorders in the positions of metal atoms are modeled using special quasi-random structures (SQS) for a set of different compositions. SQSs of Mg-Ti$\mathrm{H}$, similar to the ordered structures, give evidence for a phase transition around 20 at. $\%$ Ti. In addition, in chapter 4 calculations regarding the optical response of ordered and disordered $\mathrm{Mg}-\mathrm{Ti}-\mathrm{H}$ structures are presented. The effects of adding $\mathrm{Al}$ or Si to Mg-Ti and their hydrides are examined in Chapter 5. The results show that the added elements greatly improve the thermodynamic properties of the $\mathrm{Mg}$ $\mathrm{Ti}$ and their hydrides and make them more suitable for practical hydrogen storage applications.

In Part - B several hydrogen storage materials are considered that are suited for storage of hydrogen in molecular form. A significant advantage of molecular hydrogen storage over chemical storage in atomic form, which requires dissociation of the hydrogen bond and the formation of a hydride, is the improved kinetics.

The first chapter of Part B, Chapter 6, focuses on polylithiated carbon and oxygen molecules as building blocks for hydrogen storage materials. In the gas phase polylithiated molecules favorably bind several hydrogen molecules, reaching extremely high gravimetric densities. Practical use of polylithiated molecules is made possible by condensing them on graphene sheets. In particular B or Be doped graphene strongly bind the molecules without seriously affecting the hydrogen bind- 
ing energies.

In Chapter 7, boron planar sheets are considered as hydrogen storage templates. The computational results indicate that the alkali metal (AM) decorated boron sheets are a much better starting point for hydrogen storage than their carbon based counterparts. In particular, the boron-Li system is a promising candidate for hydrogen storage purposes.

Chapter 8 focuses on the potential applicability of metal organic frameworks (MOFs) as hydrogen storage materials. Stand-alone MOFs interact with hydrogen molecules via physisorption. Accordingly, massive operating pressures and cryogenic conditions are required to store reasonable amounts of hydrogen. Previous studies show that incorporating lightweight $\mathrm{AMs}$ or alkaline earth metals (AEMs) to the MOF structures enables a hydrogen storage under moderate thermodynamic conditions. The findings presented in this chapter, however, contradict the earlier studies on the interactions of AMs (AEMs) with the MOF systems. The results indicate that AMs (AEMs) bind very strongly to the oxide building blocks of MOF systems and have a detrimental effect on the structure of the entire system. As implied in chapter 8, lightweight AM or AEM doping of MOF structures is not an ideal strategy. 



\section{List of publications}

[1] Tunable hydrogen storage in magnesium-transition metal compounds: Firstprinciples calculations

Süleyman Er, Dhirendra Tiwari, Gilles A. de Wijs, and Geert Brocks Phys. Rev. B 79, 024105 (2009)

[2] First-principles study of the optical properties of $M g_{x} T i_{(1-x)} H_{2}$

Michiel J. van Setten, Süleyman Er, Geert Brocks, Robert A. de Groot, and Gilles A. de Wijs

Phys. Rev. B 79, 125117 (2009)

[3] First-principles modelling of magnesium titanium hydrides

Süleyman Er, Michiel J van Setten, Gilles A. de Wijs, and Geert Brocks J. Phys.: Condens. Matt.; Accepted (2009)

[4] A computational study of hydrogen storage in $\mathrm{Mg}$-Ti-X $(X=A l$, Si)

Süleyman Er, Gilles A. de Wijs, and Geert Brocks

(in preparation)

[5] Hydrogen storage by polylithiated molecules and nanostructures

Süleyman Er, Gilles A. de Wijs, and Geert Brocks

J. Phys. Chem. C 113, 8997 (2009)

[6] DFT study of planar boron sheets: A new template for hydrogen storage Süleyman Er, Gilles A. de Wijs, and Geert Brocks

J. Phys. Chem. C; Accepted (2009) 
[7] Impacts of lightweight metal doping on metal-organic frameworks Süleyman Er, Deniz Cakir, Gilles A. de Wijs, and Geert Brocks (in preparation)

[8] Role of 3d transition metals in $\mathrm{NaBH}_{4}$ thermodynamics: Ab initio study Deniz Cakir, Süleyman Er, Gilles A. de Wijs, and Geert Brocks (in preparation)

[9] Effects of Ca coating on boron fullerenes

Süleyman Er, Gilles A. de Wijs, and Geert Brocks (in preparation)

[10] Hydrogen storage abilities of lightweight metal decorated boron doped heterofullerenes

Süleyman Er, Gilles A. de Wijs, and Geert Brocks (in preparation)

[11] Ca deposited tetrazine based covalent-organic frameworks for hydrogen storage Süleyman Er, Gilles A. de Wijs, and Geert Brocks (in preparation) 


\section{Short curriculum vitæ}

Name: Süleyman Er

Date of birth: $\quad$ May $31^{\text {st }}, 1980$

Place of birth: Civril, Turkey

2009: Junior Researcher (Ph.D.) in Physics.

University of Twente, The Netherlands.

Project: Computational modeling of novel materials for hydrogen storage.

Supervisors: Dr. Geert Brocks and Prof. Dr. Paul J. Kelly.

2005: Bachelor of Science (B.Sc.) in Chemistry.

Bilkent University, Turkey.

Project: Computational study of single-site mutation and secondary structure stability: An isodesmic reaction approach.

Supervisor: Prof. Dr. Andrzej S. Cieplak.

2004: Visiting Research Scholar.

University of Illinois at Urbana-Champaign, USA.

Project: Atomic scale simulations for anti-cancer drug discovery.

Supervisor: Prof. Dr. Eric Oldfield.

2003: Trainee Research Scholar.

Max Planck Institute for Polymer Research, Mainz, Germany.

European Snychrotron Radiation Facility, Grenoble, France.

Project: Controlled design of macro ion based nanostructured materials.

Supervisor: Dr. Franziska Gröhn. 


